Barbara Lüneburg

TransCoding - From 'Highbrow Art' to Participatory Culture

Culture \& Theory | Volume 155 
Barbara Lüneburg (Prof. Dr.) is a researcher and internationally performing artist in new media art. Her research interests include performer-composer collaboration, gamified audiovisual and participatory art. 
BARBARA LÜNEBURG

\section{TransCoding - From 'Highbrow Art' to Participatory Culture}

Social Media - Art - Research

[transcript] 
The artistic research project "TransCoding - From 'Highbrow Art' to Participatory Culture" was funded by the Austrian Science Fund (FWF) as project PEEK AR 259-G21.

\section{FШF}

Der Wissenschaftsfonds.

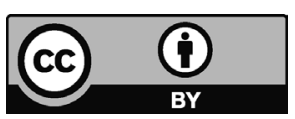

First published in 2018 by transcript Verlag

This work is licensed under the Creative Commons Attribution 4.0 (BY) license, which means that the text may be be remixed, transformed and built upon and be copied and redistributed in any medium or format even commercially, provided credit is given to the author. For details go to http://creativecommons.org/licenses/by/4.०/.

Creative Commons license terms for re-use do not apply to any content (such as graphs, figures, photos, excerpts, etc.) not original to the Open Access publication and further permission may be required from the rights holder. The obligation to research and clear permission lies solely with the party re-using the material.

\section{(C) Barbara Lüneburg}

\section{Bibliographic information published by the Deutsche Nationalbibliothek}

The Deutsche Nationalbibliothek lists this publication in the Deutsche Nationalbibliografie; detailed bibliographic data are available in the Internet at http://dnb.d-nb.de

Cover layout: Maria Arndt, Bielefeld

Cover illustration: Image of the audiovisual installation Read me (C) Barbara Lüneburg, Graz, 2017

Language editor: Sarah Patey

Printed by Majuskel Medienproduktion GmbH, Wetzlar

Print-ISBN 978-3-8376-4108-0

PDF-ISBN 978-3-8394-4108-4 


\title{
CONTEnts
}

Preface $\mid 7$

I. TransCoding -

From 'Highbrow Art' to Participatory Culture | 9

1. Introduction $\mid 9$

2. The Research Field $\mid 18$

3. The Team Interviewed by Torsten Flüh $\mid 28$

\author{
II. From Lonely Genius to Community Creation - \\ Whose Voice Matters? | 51 \\ 4. Theory of Participatory Culture $\mid 52$ \\ 5. Social Media - Content Strategy $\mid 58$ \\ 6. The Art Production | 66 \\ 7. Motivation for Participation $\mid 72$ \\ 8. The Community's Voice $\mid 83$ \\ 9. A Potential for Change $\mid 101$
}

\section{Artistic Research -} New Insights Through Arts Practice? | 127

10. Artistic Research and Ethnographic Sociology -

One Project, Two Methods 129

11. Case Study I am a Priest | 139

12. A Comparison of Discourses $\mid 159$ 
Conclusion | 169

\section{Appendix | 175}

Acknowledgements | 175

Bibliography | 177

Authors | 187

Index $\mid 189$

Annotated website:

www.transcoding.info (not included in this printed book) 


\section{Preface}

This monograph documents the artistic research project TransCoding From 'Highbrow Art' to Participatory Culture (PEEK AR 259-G21), funded by the Austrian Science Fund. Trans Coding engages with the topic of participatory culture, using social media in the context of artistic practice to involve an online audience in the making of a multimedia artwork.

In this study, I examine the social media strategies and artistic practices employed, introduce the voice of the project's community, and present and discuss both the artworks that came into being and their genesis, while reflecting on scholarly and methodological considerations. I investigate the notion of authorship, authority and aesthetics in participatory art and digital culture in the context of the project, and the motivation to contribute via web 2.0. My team colleague sociologist Kai Ginkel and I debate discourses on qualitative methodologies in artistic research and ethnographic sociology, in an attempt to compare the construction of knowledge produced by the respective fields, and we investigate methodological inferences.

Artistic research, and with it the process of doing art and the artwork itself, are at the heart of this investigation. For that reason, I offer an augmented way of reading by providing a complementary website. Designed in a way that reflects the structure of this book, it presents annotated audio and video excerpts of TransCoding's artworks, interview passages and community contributions. In addition, the website documents the connected artwork Slices of Life for violin, video and soundtrack and a playlist of different versions of the audiovisual installation Read me personalised for single community members of TransCoding (transcoding.info/english/book.html). Both have been created as part of the project and with the participation of community members. The book could be read as text alone, but in my opinion the reader will derive more and gain a more holistic experience from listening in parallel to the additional audio and visual media made available.

Parts of chapters 1 ('Introduction'), 2 ('The Research Field'), 4 ('Theory of Participatory Culture'), 9 ('A Potential for Change') and 13 ('Conclusion') draw on my paper From 'Highbrow Art' to Participatory Culture - A Potential 
for Change (2017) at RUUKKU, Studies for Artistic Research, with the kind permission of the publisher.

The artwork and this monograph would not exist without TransCoding's community. I would like to thank all our active and passive supporters; by name, I am grateful to Louis Aguirre, Victor Barceló, Julien Charest, Tamara Friebel, Gloria Guns, Anthony Green, Camilla Hoitenga, Olivia Kieffer, LIA, Feliz Anne Reyes Macahis, Katarina Michelitsch, Anahit Mughnetsyan, Alina Murzakhanova, Paul Norman, Heghine Ohanyan, Mikolaj Pociecha, Alexandra Radoulova, Hali Rey, Damian Stewart, Felix Christian Thiessen, Ricardo Mateus Tovar, Sabina Ulubeanu, Isabelle Vigier, Michael Wolters, Susanne Wosnitzka, and my students in the seminar 'Undoing Gender' from the University of Music and Performing Arts Graz and Karl Franzens University of Graz, who all contributed with voice, sound, image or word to the artworks Read me or Slices of Life.

- Link to the video documentation of Slices of Life:

http://transcoding.info/english/book.html - PREFACE 


\section{TRANSCODING - FROM 'HIGHBROW ART' to PARTICIPATORY CULTURE}

\section{INTRODUCTION}

K-Pop artist PSY and gender theorist Judith Butler, contemporary art music and pop culture - how can these possibly be considered together? The artistic research project TransCoding - From 'Highbrow Art' to Participatory Culture has attempted to find an answer. TransCoding engaged with the topic of participatory culture by using social media in the context of artistic practice. My team and I encouraged participation and shared discourse in the new arts by actively involving an online audience in the making of a multimedia artwork. We hoped thus to make 'highbrow' art, that is, contemporary classical music and multimedia art, more accessible to a broader public.

Our main target group was an internet-literate young audience, mostly drawn from popular culture, who might not necessarily be considered the typical audience for classical contemporary multimedia performances. The blog at https://what-ifblog.net served as the central social media and content base, and also as a contact point for our community. Community members were directly involved in the genesis of Trans Coding's artworks via their creative contributions. TransCoding facilitated interactions between the participating members and the professional artist(s) through calls for entries channelled via the project's social media hub.

In Trans Coding, artistic research went beyond the investigation of the artistic process, and expanded into new contexts. By offering participatory culture via web 2.0 as part of our arts project, my team and I invited contributors to speak out, and to share a discourse about and exert influence on two major arts projects. We employed principles of participatory culture in the communication and creative process, thus redefining the (commonly hierarchic) relationship between artist and community as one of permeability and mutual influence. We applied findings and theories from media sociology and cultural science to 
an artistic process; we investigated their applicability and meaning in the arts, and their impact on the resulting artworks itself, on the community we had gathered, and on the role of the artist.

\section{The Context}

From February 2014 to February 2017 , my team and I built and maintained a network of various social media channels around a main hub, the WordPress site what-ifblog.net. Here we presented our topics - multimedia art and contemporary art music, community participation and the creation of our show - under the categories 'Art we love,' 'You, Us and the Project,' and 'Making of,' respectively. In a fourth category we chose 'Identity' as our main topic for the content of the artwork and the blog (Fig. 1.1).

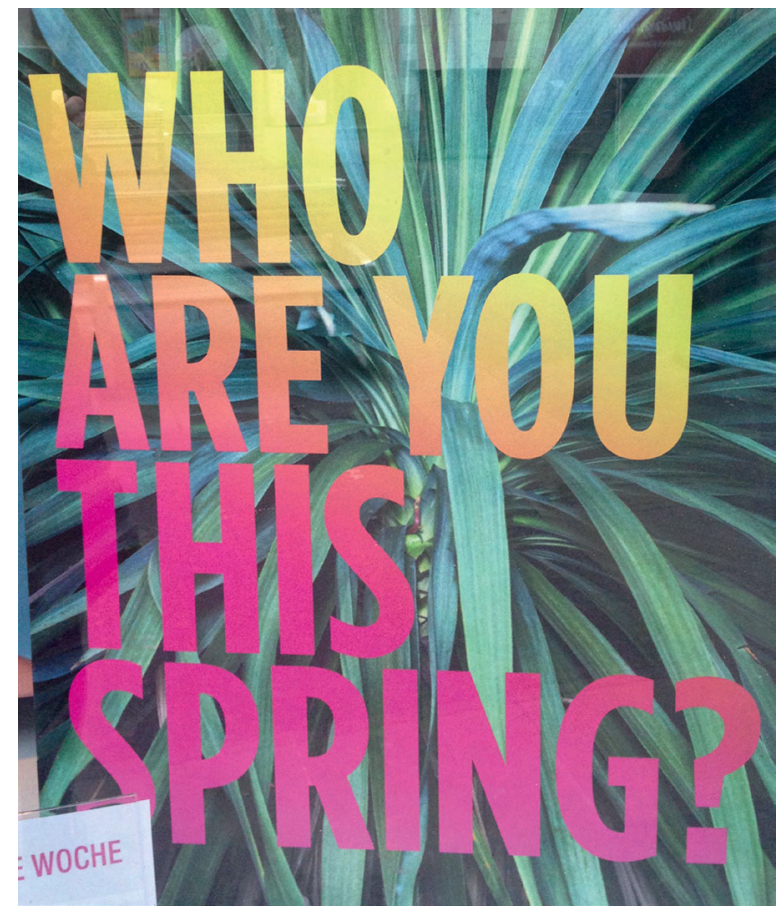

Fig. 1.1: The artistic topic of TransCoding was identity. In one of our first calls for entry we asked the community members about their 'spring identity'. Image (C) Barbara Lüneburg

TransCoding was for women and men, professional artists and home producers. The concept of identity offered a framework for the project that is universally 
relevant and unites our otherwise diverse community members. All were invited to participate in the community through any (feasible) means of expression: music of all genres, poetry, prose, drawings, photos, videos, interviews, links or ideas. Our main target group was comprised of digital natives who were technically savvy, aged between twenty and thirty-five, and were interested in expressing themselves creatively while coming from popular culture. However, participants outside of the target group were equally welcome to join the community (Fig. 1.2).

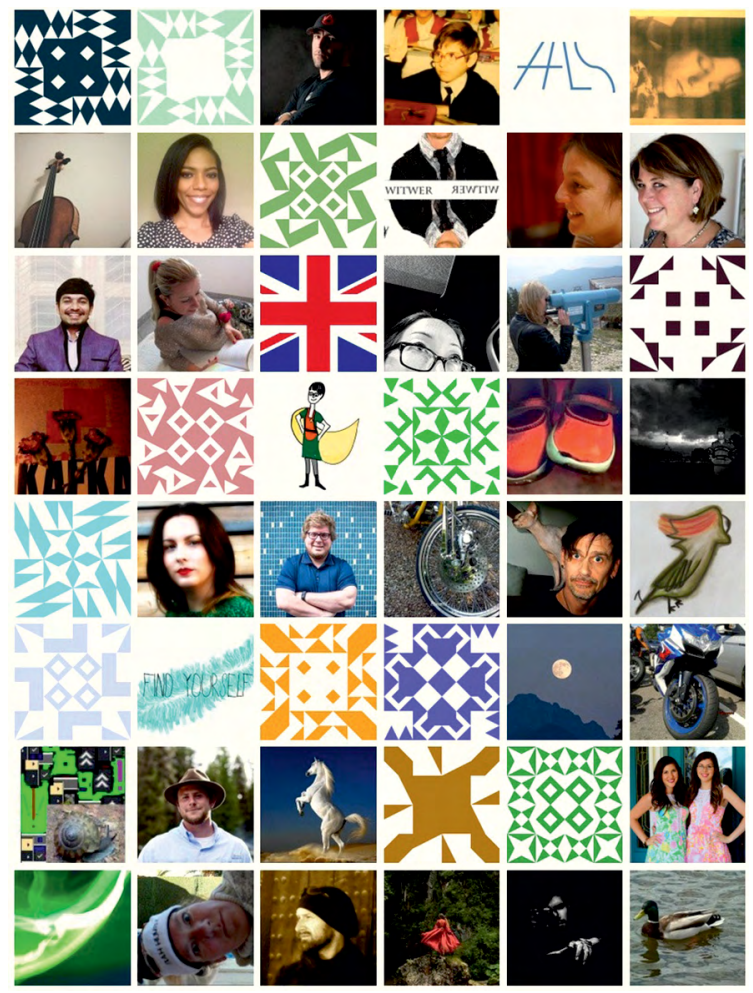

Fig. 1.2: Our community on the main hub http://what-ifblog. net. Within the first two years of TransCoding a network of almost 1200 people developed across our social media channels. Image (C) Barbara Lüneburg

The blog was our main contact point with our community, and afforded them the opportunity to participate in our project. Via calls for entries we encouraged our visitors to contribute images, sounds and texts that I incorporated in the artworks. Through our social media channels we invited them to interact and exert influence on the creation of the evolving artwork, and additionally 
presented situations and material for community members to create their own artwork (whether or not it was closely related to TransCoding). We raised interest in participation in art as a way to express one's identity and achieve personal empowerment, and we fostered a sense of community through belonging to a peer group participating in an arts project.

We gave our community members authority to shape our work and offered them a platform to meet and make their interest clear. As we invited contributors to exercise influence over the joint artwork, we looked at change as viewed through the power relationship between artist and community.

\section{The Inspiration}

Where did the idea for Trans Coding come from? At least three major influences inspired the project. Many readers will remember Gangnam Style, the viral 2012 YouTube hit by the Korean pop artist PSY (http://bit.ly/2ud4afx). Only a few days after the release of Gangnam Style on YouTube, fans from all over the world had created covers and parodies, and launched remixes that replaced PSY's Korean theme with their own life and identity. The young digital social media public, it seemed, was obsessed with Gangnam Style. Of course, in the background of this huge success, the South Korean label company behind his song, YG entertainment, had invested in it with a well-structured, well-prepared and fastidiously executed campaign. (Drum 2012) They were able to tap into an incredible potential for creativity and for communication among people from different cultures and countries on social media. Although it was clear that as a team working in new (classical) music we would never be able to campaign for our purposes in the same way as a pop label operating on an international scale, the phenomenon of Gangnam Style became one of my inspirations for the use of social media in TransCoding.

At the same time, I taught seminars on film music, audio branding and media theory at media universities in Germany and Austria. My students were tech-oriented young people whose creative background was popular culture. Their thinking and creative activity was quite different from mine with my artistic practice originating in classical and contemporary art music. In seminars we explored with interest our different worlds and had critical discussions on Luigi Nono and K-Pop, Henry Purcell and social media, or audiovisual installations and electronic avant-garde pop music. I loved the music they made from what I offered them and I realised that I could actually lure people into contemporary art music just by introducing them to it and sharing my knowledge, enthusiasm and passion for it. In their works an intriguing mix of popular music and contemporary art music emerged and my students started to adopt new artistic perspectives. I too began to think in 
new ways. The reciprocal interaction between me and my students changed my approach not only to my own domain, but also to the field they came from, popular culture. We mutually influenced each other's artistic practice. Those students were my model when I thought about the target audience for TransCoding - From 'Highbrow Art' to Participatory Culture.

Last but not least, I was inspired by philosopher and gender theorist Judith Butler. One day in spring 2013 , I was doing research for a performance set-up for the artworks of TransCoding. When I was randomly sifting through YouTube videos, I found a short interview with Judith Butler in which she talked about the notion of gender performativity. She explained what it means if we say 'gender is performative':

"for something to be performative means that it produces a series of effects. We act and walk and speak and talk in ways that consolidate an impression of being a man or being a woman. ... We act as if that being of a man or that being of a woman is actually an internal reality or something that is simply true about us, a fact about us, but actually it's a phenomenon that is being produced all the time and reproduced all the time, so to say gender is performative is to say that nobody really is a gender from the start. ... It's my view that gender is culturally formed, but it is also a domain of agency or freedom." (Butler, Judith Butler: Your Behavior Creates Your Gender 2011)

If we refused to perform the gender script that is handed to us by society, we could instead perform a different, self-chosen script; ideally, we would decide on our gender (on a daily basis) and pick the life that suits us best.

This notion of free choice sparked the idea of creating a multimedia show with a series of short movements of three to four minutes - similar to the format of a pop show - traversing a whole palette of musical possibilities: from classical music to pop music, from pop music to the new music avant-garde, or perhaps from new music to metal. Each song would feature a different story and a different kind of identity. I had found my topic for TransCoding and concomitantly for the future multimedia show Slices of Life that became one of the participative artworks developed within the project.

\section{The Community}

'Identity' seemed to me ideal for TransCoding, since it was necessary to find a subject that people coming from 130 different countries (according to the 2017 WordPress statistics) would care about enough to start communicating and interacting with us online; we needed something that would touch them emotionally, and that was connected to their individual life, something that triggered their wishes or visions. We didn't treat 'identity' as a scholarly 
paradigm to be investigated but rather as a means to spark off the phantasy of our diverse community members and to unite individuals from different cultural backgrounds in art. Each community member, whether male or female, religious or atheistic, from East, West, North or South, young or old, could contribute from their life, culture and social context. Each song, each story, each image and each sound in the context of our project would stand for differently 'performed' identities.

\section{"What if?}

a participatory arts project about identity

a question • a creative endeavour • a research project • a community

We are an international team of artists, musicians and programmers building an online/ offline community through which we are exploring the concept of identity.

We are looking for your input while developing two different artworks, both dealing with our topic:

- An audiovisual installation that features texts, recordings, music and images from the community and from us.

- A multimedia show for violin, electronics and video that merges community contributions with our approach to art.

In the section 'You, Us and the Project' you will gain insight into our thoughts and we hope for your comments and inspiration.

\section{Contribute: How can you participate?}

If you like to make music, if you take pictures wherever you go, if you love to write or design, if you are a camera freak or an all-night creative programmer, or if you are just interested in personal freedom of choice, then What if? is for you.

Send us ideas, links, photos, music, films, texts, interviews that pick up on our topic: commercials you love, ads you hate, quotes that inspire you and links to art works you have found or you have maybe made yourself. We would love to hear from you.

These are some of our current questions to get you inspired:

What if we could live our lives regardless of the expectations of society, family, friends or colleagues?

What if our body, our gender wasn't our limit?

What if we took the freedom to be who we want to be, and how can we express this in our art?

What if? is limited. It will exist for three years. We started on 1st of February 2014 and will finish the project in 2017. During that time, we will continuously work on the blog. We will throw ideas at you and wait for your comments and replies." (Lüneburg, What-ifblog.net | About 2014) 


\section{The TransCoding Team}

TransCoding was carried out by an international team from Germany, Austria and Canada supported by the Austrian Science Fund FWF (PEEK AR 259-G21) and located at the University of Music and Performing Arts in Graz (Austria). Our research was transdisciplinary and drew on the fields of artistic research, sociology and social media expertise. The project began in February 2014 and lasted three years in total. During the first year we worked in a team of four artists and a social media strategist with shared responsibilities in the creation of the artwork. After a year we continued in a smaller team for reasons particular to the project and based on the topic 'authority for the community versus sole creative sovereignty for the artist' (investigated in more detail in chapter 9 'A Potential for Change').

From the second year on, our team consisted of two main operators. I headed up the project in the capacity of conceptual leader, social media content producer and strategic mastermind behind the communication with the community, as well as lead artist, performer and researcher. My co-worker, Clio Montrey, who was in charge of the everyday communication with the community, constantly fed various social medias channels we had installed around our main hub, the WordPress blog what-ifblog.net. We were given occasional support by a visual artist, software programmers and a dramaturge. We were later joined by Kai Ginkel, a researcher who reflected on the project from a sociologist's point of view, whose perspective was primarily shaped by his ongoing work in sociological ethnography. His prior research was concerned with a sociological understanding of sound, using the field of noise music as a case study and reflecting his own artistic involvement in that particular field in the process. Consequently, his work in TransCoding is related to artistic research while being rooted in sociological thinking.

\section{The Research Questions}

The name of the artistic research project TransCoding - From 'Highbrow Art' to Participatory Culture implies that we converted an art form commonly seen as intellectual or at least rarefied in taste into a representation of art that is derived from audience participation and thus accepted and embraced from the start. In order to get people to participate who under normal circumstances would be very unlikely to connect with the kind of contemporary 'highbrow' art we represented, we applied a popular communication tool, social media. The overriding research question was: how can we achieve the involvement of an audience not previously available in the creation of a classical contemporary artwork, and what would be the artistic and cultural social result? 
Alongside this main question, a series of secondary questions emerged from the specific setting. This will be tackled in different parts of the book: questions dealing with the building of a community via web 2.0, with the assignment of authority in the creative process, and with aesthetics and crossover art based on the exchange between community and artist. We initially asked ourselves which method would allow us, using social media, to establish a community that would pick up on the topic of new media art and crossover culture, and would want to be actively involved in the creation of an artwork.

At the same time, we deliberated whether we would be able to establish a crossover between high art and popular art by offering creative and intellectual incentives to participate in our project, while on the other hand listening back to and channelling the community's own creative voices. We asked about the interchange between the global community and the research team and what it entailed with regard to the project and to the artworks. Would we through it - be able to create an artwork that made contemporary art more permeable and accessible for the audience? When we subsequently investigated whether the interaction with the community had led to a fruitful interactive exchange between the contributors and the artist, while adding authority to the community, we also asked how in consequence it changed the role and selfconcept of the artist.

However, due to the widespread global and virtual setting of the project there was one initial question we did not have the means to answer: whether an initially unavailable audience would be willing to become an available audience in a real concert setting through participation in our artworks via social media.

\section{Summary of the Individual Chapters}

Part I of the monograph ('TransCoding - From 'Highbrow Art' to Participatory Culture') consists of a general introduction to TransCoding in chapter 1; chapter 2 describes the research field and gives an overview of participatory art in the field of contemporary (classical) music and the project-specific methodology. Part I is rounded off in chapter 3 by an interview of the TransCoding team that offers an overview of the project from the perspective of the cultural scientist Torsten Flüh. In his questions, he touches on the (crossover) aesthetics of TransCoding, the challenges of a digital society, social media in the arts and the creative process behind the project.

Part II ('From Lonely Genius to Community Participation - Whose Voice Matters?') is concerned with theories and concepts related to participative art and the theoretical framework for our social media and content strategies. In chapter 4 , I define participatory culture via web 2.0 in the context of our project. In chapter 5, I look into the social media content strategies that furthered and 
unlocked the creativity of the community for mutual benefit. In chapter 6, I recount the production of the artwork and the problems that arose out of the participatory character. Chapter 7 discusses the motivation for participation that let people contribute to the artwork of TransCoding. In chapter 8, which is dedicated to the community of TransCoding, I document selected community contributions and reflect on them. Chapter 9 ('A Potential for Change') deals with the impact of the community on the role and the self-concept of the artist.

Part III ('Artistic Research - New Insights Through Arts Practice?') offers a comparison of discourses in the fields of ethnographic sociology and artistic research. We deploy here the philosophical paradigm of Foucauldian discourse analysis. I discuss with the sociologist Kai Ginkel, co-author of the chapter, the similarities and differences in the methodologies of artistic research and ethnography. We compare the overall production of knowledge, point out similarities and differences, and search for the values, taboos and power strategies behind methods and approaches (chapter 10). We present and analyse the case study I am a Priest, a movement from the artwork Slices of Life, from the perspective of each field. Here, we investigated how a method has bearing on the 'truth' that is being sought and how it is accordingly depicted (chapter 11). Finally, we evaluate whether both disciplines might possibly inform each other in terms of methodological innovation and enhancement (chapter 12).

I conclude in chapter 13 ('Conclusion') by discussing the viability for future arts practice of the model of TransCoding, i.e. engaging the audience via online participation in a contemporary artwork. In doing this, I take into account the collaborative aspects of the task, their social and psychological connotations and their artistic consequences. I critically assess the practicability of social media for the interaction between artist and community and address the topic of crossover art. I reflect on my personal artistic work in the project, how it influenced my self-concept of being an artist and why artistic research was conducive to achieving the project's research objectives and methodology.

Chapter 14 (accessible via the annotated website: www.transcoding.info) offers an online documentation of the artworks of TransCoding, Slices of Life for violin, soundtrack and video and the audiovisual installation Read me. I created both these artworks in exchange with and including contributions from the TransCoding community. The website also includes interview snippets and additional artworks that came into existence within the framework of the project, all of which are discussed throughout the monograph. 


\section{THE RESEARCH FIELD}

Note: this chapter is adapted from an article submitted to the Finnish online journal on artistic research RUUKKU: Lüneburg, From ,Highbrow Art' to Participatory Culture - A Potential for Change 2017.

Our methodology was based on qualitative research. We conducted a concurrently artistic and ethnographic research study in which the fieldwork took place primarily but not exclusively in the virtual realm of web 2.0 , on our social media platforms, with a focus on our central online hub, what-ifblog. net. As the lead artist, I myself especially acted within the field of participatory art. What follows is a short overview of the methodologies of artistic and ethnographic research applied in this project, and of participatory art within the domain of classical contemporary music.

\section{Artistic Research - The Central Artworks and the Influence of the Community}

With TransCoding I embraced not only the final artworks but also the process of producing art as part of my investigation: the gathering of inspiration, the communication process and artistic exploration. As artistic practices are always embedded in context and not detached from society or time, I put an emphasis on the work with the community and on the situation jointly created on our social media platforms. The outcome of the interrelation between community and artist flowed into the body of the art and the reflection on it. I viewed the arts practice and research as performative, meaning that both the artwork and the creative process modified how I and possibly our participating community understood and reflected the world; thus the shared arts practice did something to us, it 'performed' us and we performed through it coming from a material to an immaterial level in our artistic and perhaps even personal identity.

What are the artworks I am referring to? The entirety of TransCoding's social media channels around the main hub what-ifblog.net was a composite work produced jointly by our community and the project team. It formed one of the central artworks. Here the interaction between all stakeholders stood in the foreground. Participation was open to everybody, with limited and only very occasional curation by the TransCoding team. The different online channels served as the means of access to the project for our worldwide community, as communication platforms and as communal online exhibition spaces for mini artworks by all stakeholders. It served also as an artistic documentary of the interaction between the team and its community. We considered it a common contextual art artefact that revealed the connections between TransCoding's art works and the conditions of their production. 
A further central artwork was the multimedia show Slices of Life for violin, soundtrack and video. It was created by me as the lead artist, and it incorporates community contributions for which we called via calls for entry. (The reader will find a detailed description of the participation mode along with a presentation of selected community contributions in Part II 'From Lonely Genius to Community Creation - Whose Voice Matters?').

With the third main artwork, the interactive audiovisual installation Read me, I provided a technological setting and a conceptual frame that could be filled with the personalised content of individual community members. Here the development of the artistic content could be entirely authored by a single community member.

Additional satellite artworks were produced in workshops around TransCoding in which non-professional participants shaped their own work around the topic of identity. Participants had a say in the development of an overall performance or personal artwork, while I as TransCoding's lead artist offered an optional initial conceptual framework and assisted with professional advice in the realisation of the artwork.

\section{The Researcher Selves}

Our operating mode, based on communication through social media, has required us to engage on an almost daily level with members of our community to establish trust, acceptance and the feeling of authenticity. It was therefore of crucial importance that the social media manager Montrey and I were constantly present on the various social media platforms. So we concurrently immersed ourselves and actively participated in the field that we investigated. As for our position in the field, I follow the definition of sociologist Shula Reinharz (1997, p. 5, cited in Sandiford 2015) who identifies a number of researcher selves for field-workers. My co-worker Montrey and I appear in three roles that are not always separable from each other: the role of the "brought self," the "situational self" and the "research based self." Research based selves relate "specifically to the research role (e.g. being an observer)", brought selves "are more personal and provide a sense of individuality" and situationally created selves "may or not be related to the research project (e.g. being a temporary member of the studied group)". (Sandiford 2015)

The role of the 'brought self' was evident when I, in my capacity as head of project, functioned as conceptualiser of the overall project; when my colleague Montrey and I set topic of the overall artwork, 'identity,' fed it, and shaped the way in which art an identity were introduced to our community; when we both formulated the calls for entry that invited the community to contribute to our artwork. (Figs. 1.3 and 1.4). 


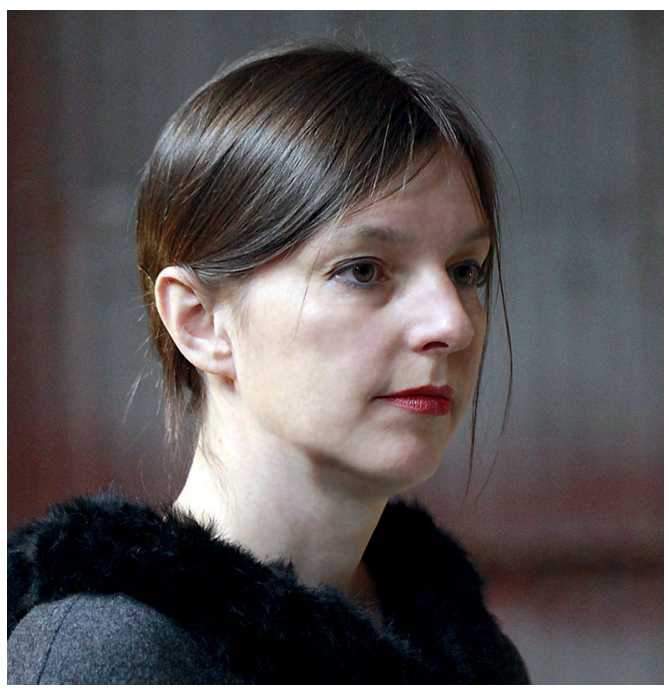

Fig. 1.3: Brought self: Head of project Barbara Lüneburg in the capacity of conceptualiser of the project, communicator on the social media channels and lead artist. Image: Courtesy of Mihai Cucu

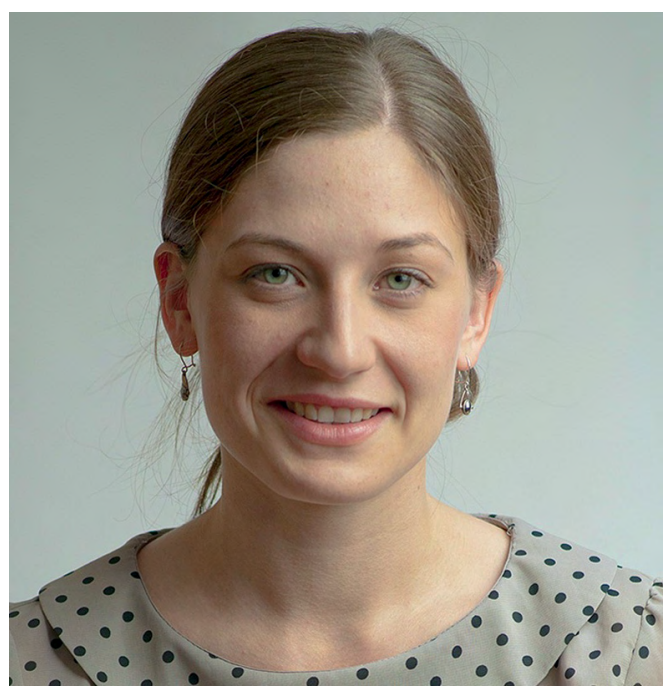

Fig. 1.4: Brought self: Social media strategist Clio Montrey in the capacity of blogger, communicator on the social media channels and networker. Image: Courtesy of Unison Shot 
My personal 'brought self' was likewise relevant when I worked as composer, visual artist or performer within the project, fulfilling my role as lead artist and concurrently artistic researcher. I created the framework of the audiovisual installation Read me as well as the multimedia performance Slices of Life for violin, electronics and video. The material I used for both artworks was based, in part and to varying degrees, on contributions from our community. I curated the contributions that were included in the artwork while searching for an adequate framing, which allowed our members to identify with the project, and featured their contributions in an authentic and respectful manner. In both contexts, our personal selves and my individual self as artist were visible and provided a sense of authenticity, which we sustained through blog posts in the category 'Making of.' Under this heading our community could read what went on behind the scenes of TransCoding. Through these 'journal entries,' we shared our experiences, challenges and ideas while putting the collective artwork together.

The role of the 'situational self' appeared when Montrey and I took on the role of temporary members of the overall social media community, acting as bloggers participating in challenges on https://dailypost.wordpress.com/, as SoundCloud members who participated in 'February Album Writing Month' jointly with our community members (http://fawm.org/); or when we roamed Facebook or Twitter for inspiration. In short, we entered this mode when gathering, learning, sharing, acknowledging, and narrating along with our community without taking on a leading role with any kind of preconceived authority but rather 'to get the feel of what it is like to be a part of the community.' (Figs. 1.5 and 1.6). 


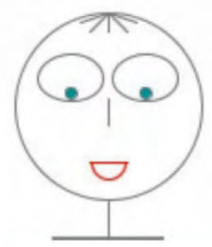

\section{Mad Maddy 0}

@madmaddy

จ Austria (O Feb 2015

what-ifblog.net $\mathbf{0} \boldsymbol{\bullet}$

\section{Artist Bio}

Influences: I am happily influenced by new music and new media art. I love glitches as much as I love the violin. Modular synthesizers are favourites of mine. What else? I like a lot of different styles.

\section{Mad Maddy}

Bring on the music. It helps my coder brain process your lovely sounds into good and evil algorithms!

Fig. 1.5: Situationally created self: Snapshot of Barbara Lüneburg's identity as Mad Maddy on the February Album Writing Month challenge (FAWM.org). Image (C) TransCoding

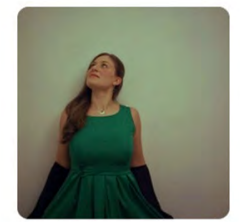

\section{Clio Em 4}

\section{@clioem 则}

P Canada (1) Feb 2015

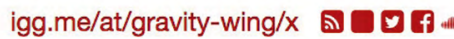

\section{Artist Bio}

Influences: Lana Del Rey Leonard Cohen Björk Simon \& Garfunkel Fever Ray Yann Tiersen Bob Dylan Dolly Parton Adele Beyonce Bach Edith Piaf Stevie Nicks

In 2015, I wrote Gravity Wing.

This year I release the final novel and album (have a look at my Indiegogo site: http://igg.me/at/gravity-wing/x it's all very nifty, I promise!)

Fig. 1.6: Situationally created self: Snapshot of Clio Montrey's identity as Clio Em on the February Album Writing Month challenge (FAWM.org). Image (C) TransCoding 
In the role of the 'research based self', we conducted an overt study in the sense that it was known to our community that they were part of a research project. We observed, reflected, analysed and drew conclusions as to communication strategies, community motivation and the community's influence on the artwork and on the artist(s). From an artistic standpoint, my personal research was focused on how I could incorporate the community's contributions and influence in an authentic, respectful and yet artistically interesting way into the overall artwork. Furthermore, I traced how the interaction with the community changed myself and my art. I discussed the findings with the academic field and society in general in talks, workshops and papers. Sociologist and team member Kai Ginkel took on the role of the passive participant observer. He was present in the field but took part neither in the artistic activity nor in the communication process with the community. Instead, he compared the use of the methodology of artistic research in the project to methodologies of his field of practice, ethnographic sociology.

I was the only member of the team to take on the role of the 'artist self' (Fig. 1.7). As a performer I came from yet another context and peer group, namely that of the contemporary music scene. This scene differs slightly in each country according to cultural politics and historical background; it influenced me while composing and when offering the show to festival promoters.

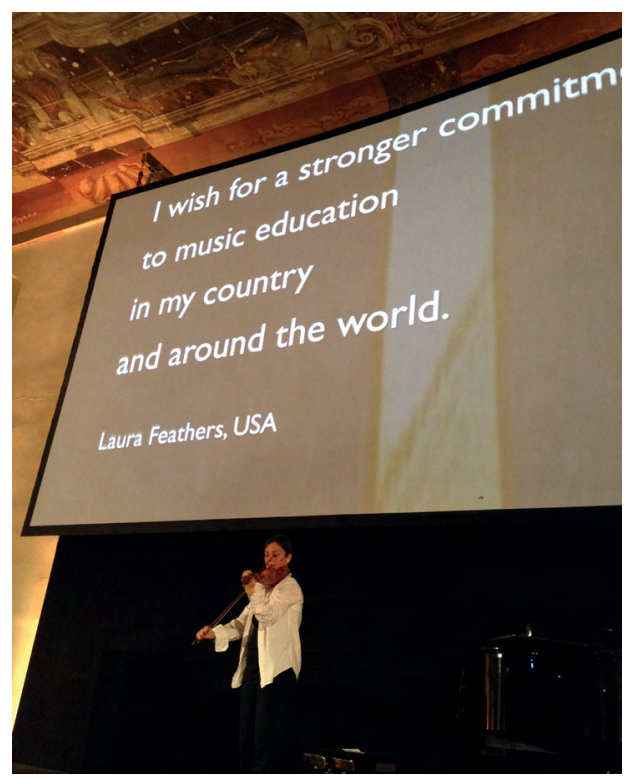

Fig. 1.7: Artist self: Barbara Lüneburg performing What if we had wings?, the first composition developed in the framework of TransCoding, in Vienna at European Researchers ' Night 2014. Image (C) TransCoding 
Link to the video documentation of What if we had wings?:

http://transcoding.info/english/book.html - PART I CHAPTER 2

\section{Participatory Art in the Field of Contemporary (Classical) Music}

Using the work of art historian Claire Bishop as a lens, I position TransCoding in the field of participatory art meaning "the involvement of many people (as opposed to the one-to-one relationship of interactivity)" in the sense of "a reconsideration of the ways in which art is produced, consumed and debated". (Bishop 2012: 1 and 3) We didn't conceptualise TransCoding as primarily politically motivated art (as socially engaged, educative or associated with the general political question of the rights and voice of the citizenship.) Instead we delivered the framework for an arts project and produced situations in which our community could be creative together with us or independently from us, express their voice and enter into discourse.

The artistic and aesthetic field in which our project is contextualised is classical contemporary, music-based multimedia art. In this project we are concerned with through-composed electro-acoustic (multimedia) music with a performer. Within this field only little participatory art can be found, usually in audio art or soundscape art, some of which are described here.

\section{Stereopublic: crowdsourcing the quiet}

This soundscape project was devised, directed and composed by sound artist Jason Sweeney. He asked its participants to seek out quiet spaces in a city and share visual and audio impressions online. On request these recordings were turned into compositions:

\footnotetext{
“'Stereopublic: crowdsourcing the quiet' is a participatory art project that asks you to navigate your city for quiet spaces, share them with your social networks, take audio and visual snapshots, experience audio tours and request original compositions made using your recordings." (Sweeney 2012)
}

The project had documentary features, the participants contributed information and material but did not participate as composers themselves.

\section{Personal Soundscape Project}

The Personal Soundscape Project of the ORF festival musikprotokoll and ORF Kunstradio, both hosted by the Austrian public broadcast service (ORF), encouraged people to engage online with an audio art project. 
"As part of an online art project initiated by musikprotokoll, for months, school-goers ... and audio recording enthusiasts were collecting sounds, listening in their everyday lives, exploring and recording their personal soundscapes: they have now processed, layered and arranged the sounds of their lives and translated them into art. The result is a kaleidoscope of 'Personal Soundscapes' and at the same time a young sound image of the province, which can be heard and modified online on the project website. ORF Kunstradio embarks on a journey through the mountains and valleys of these acoustic landscapes."

(ORF 2013-2015)

The project was concurrently participative and educative. Participants could either simply collect and contribute sounds or take it one step further, creating their own composition. Some of these works were then featured at the musikprotokoll festival.

\section{Walk that sound}

In Walk that sound by Serbian artist Luka Ivanovic, aka Lukatoyboy, the artist provides walkie-talkies to participants so that they can record conversations and environmental sounds coming from a nearby area. Lukatoyboy collects the material and from it creates a recording that is later broadcast via radio stations. The project has the character of a happening and at the same time of a documentary.

\section{Blind Tapes}

For his ongoing participatory project Blind Tapes Ivanovic invites four people to individually record ten minutes of playing their instrument, singing or talking on a 4 -track cassette recorder. The recordings overlay each other. The people are unaware of the people who play before, or will play after them. Ivanovic later sells a mix-down of the recordings under the name Blind Tape Quartets mentioning the individual participants.

"A small, basic studio with a portable 4-track cassette recorder is provided for musicians (established, amateurs, first-time...) and/or singers (writers, spoken word artists...) to record a single channel, until a recorded 'quartet' is ready and blind-mixed. Each person is responsible for the of the overall, 4 -track/channel recording - and will be provided with some directions in order to preserve the overall mix from being too busy." (Ivanovic, Blind Tape's quartet biography 2012)

\section{Disquiet Junto}

At Disquiet Junto, an "association for communal music/sound making" participants hosted by webzine publisher Marc Weidenbaum, USA, follow the given theme of the week and produce a song that is not part of an overall artwork. The only connection between the contributions is their being featured 
on the Disquiet Junto SoundCloud and their individual engagement with the weekly topic.

"The Disquiet Junto is a group based on Soundcloud.com where musicians respond to weekly, fast-turnaround assignments to compose, record, and share new music. The idea is to use restraints as a springboard for creativity." (Weidenbaum 1992-2017)

The community can meet in an online forum to discuss, reflect and exchange ideas (http://disquiet.com/forums/categories/disquiet-junto). The project encourages and gives incentives to compose, it offers a platform for exposure, discussion and reflection for the community, but it does not connect the participants through an overall artwork.

\section{WIReless}

For his project WIReless - Ein Social-Media-Klavier-Recital mit Martin Tchiba [WIReless - A Social-Media-Piano-Recital with Martin Tchiba], pianist and composer Martin Tchiba connected participants online and through the event of a piano recital. For this concert he commissioned established composers to write short piano works for him and combined their works with miniatures by composers he assembled via social media. Additionally, he invited school classes and students from music schools to take part and contribute works one minute long. The artists were invited to interact with each other via Facebook or Twitter, and - if they wished - to relate to the work of their predecessors in the process. During the actual live concert, tweets by the audience were projected on a screen, to which the pianist reacted with improvisation. Additionally, spectators could trigger selected sound samples via their smartphones and thus influence the overall atmosphere. Although Martin Tchiba emphasises that he instigated a public opinion poll in which he invited the audience to vote for their favourite pieces, he also makes it clear that he had the final curational decision on every piece that was featured in his recital.

Tchiba states that in his project "the relation of the individual versus the collective and the democratic participation in decision-making in art was likewise reflected." (Tchiba 2017) However, according to him half of the pieces performed were commissioned (other than collected via social media) by Martin Tchiba himself from professional composers he knew and trusted. The additional works by school classes were developed under his lead using his compositional framework, and his own improvisation formed another $11 \%$ of the overall programme. Furthermore, he had final curatorial authority over the rest of the programme, which was gathered via social media. Hence I would suggest that his model of participatory culture was what Nina Simon calls 'contributory,' where participants are "solicited to provide limited and specified objects, actions, or ideas to an institutionally controlled process." (Simon 2010: 187) 
In summary, I can state that within the field of participatory contemporary (art) music, I have not found a single work comparable to TransCoding in the way we encompassed participation, exchange between artist and community, the permeability of authority and the building of a community.

The following chapter provides an overview of the project on basis of an interview conducted by the cultural scientist Torsten Flüh with the TransCoding team. Flüh, a scholarly blogger himself, is interested in the challenges of TransCoding's digital community, social media in the arts in general, the creative process behind the project, and in how the crossover aesthetics of the project transferred onto and influenced the final artwork. 


\title{
3. THE TEAM INTERVIEWEd BY TORSTEN FLÜH
}

\author{
Interviewer: Torsten Flüh (TF) \\ Interviewees: Kai Ginkel (KG), Barbara Lüneburg (BL) \\ Clio Montrey (CM)
}

\section{Performance of Slices of Life - The Shirt}

TF: Barbara, we met for the first time at the New Music Festival Ultraschall in January 2016 [on the occasion of Barbara Lüneburg's solo recital on January 24, 2016]. Your multimedia performance has left a strong impression on me. Part of your recital was the composition Slices of Life - The Shirt, generated from what-ifblog.net. Please, tell us how it happened that this piece was included in your programme.

BL: When the promoter of Ultraschall approached me in 2015 , he asked me to conceptualise a multimedia solo recital under the topic 'politics,' 'ritual' or 'identity.' At that time, I was already working with the TransCoding community on the multimedia show Slices of Life and I was searching for an opportunity to give the first section of it, The Shirt, a test run with my peer community.

The Shirt is the introductory sequence of Slices of Life. It tells the story of a person who is in search of his or her own identity; it could be a woman or it could be a man. She borrows other people's clothes and wears them for some time because - I'm quoting the story - "She feels she doesn't have any specific identity herself, and therefore she likes the idea of wearing other people's clothes so that she could (in some way) step into their identity and discover what it might be like to be them."

Link to the video documentation of The Shirt:

http://transcoding.info/english/book.html - PART I CHAPTER 3

To me this was the perfect introduction to the topic of identity, somebody searching for his (or her) personality and defining him- or herself through other people's clothes. I understand this ritual of putting on a different identity - that is defined simply by your choice of clothes - as a symbol of the freedom you have; that somehow you can choose and express who you want to be by regular, everyday actions. I loved the playfulness which stands behind this community story, and the underlying quest for identity. I see The Shirt as an entry point into what I wanted to depict in this show and also on the social media sites, and to me it perfectly fitted into the given topics of Ultraschall. 


\section{The Aesthetics of Slices of Life}

TF: In your research project, composition, blogging and gender studies overlap and are jointly expressed in the show Slices of Life. You work with a variety of media, for instance social media such as a WordPress blog, Facebook or Twitter, and artistic media such as text, image, film and sound. You interweave them in the investigation process and in the creative outcome. It constitutes a multiplicity of structures and inputs. Therefore, my next question would be this: how is this linked to your topic of identity and, furthermore, to the aesthetics you use?

BL: This is an intricate question. Let us perhaps start at the very beginning. 'Identity' as the general subject came up because I needed to find a topic that was relevant to all the people in my community. If I had spoken about art, architecture or poetry, it would have been relevant only for a certain spectrum of people, but not for all of them. Whereas at least at some point in each person's life, the question of one's identity is important for everybody. So the topic of identity was important to me for the purpose of building a community and building interest within this community.

Coming back to your question: Yes, I would say different sorts of media definitely influenced the aesthetic of the project. Take for instance social media: each type of social media we used asked for different treatment and practice. On Twitter you express yourself within the limit of 140 characters; it's a micro-blog that "allow[s] users to exchange small elements of content such as short sentences, individual images, or video links" (Kaplan and Haenlein 2011 : 106). Instagram is about visual sharing, you can upload photos and videos up to one minute long. It comes with filter and editing features to apply to both videos and photos. The social network Facebook supports people in creating a personal profile on which they can post photos, videos, and texts of micro to medium length, and connect and exchange with their friends. Often the posts are of a personal or at least semi-personal nature, serving the purpose of status updates. Then again on a blog such as what-ifblog.net, you can add more depth and length to your stories and content may range from professional to commercial or to personal. So yes, every platform we employed had its own characteristics and its own target group and each thus shaped in its own way the aesthetic of the contributions we received.

TF: Let's focus in more detail on Slices of Life and its specific aesthetic. From what I saw at the festival Ultraschall, The Shirt is comprised of typed letters, animated text, sound and slightly blurred images. Who shaped these specific aesthetics? The community through their contributions? You as the artist who produced the final video? Or maybe a combination of both? 
BL: The images I used in The Shirt came from community member Katarina Michelitsch, who contributed a film sequence of dancing hands. It was her way of communicating 'identity' in the context of emotion and bodily expression.

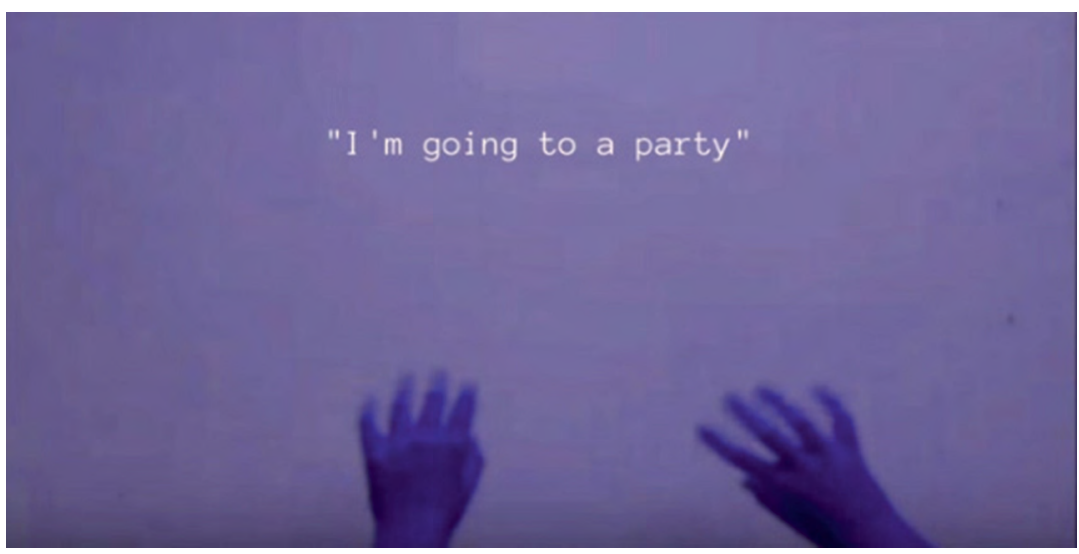

Fig. 1.8: Example of the imagery by Katarina Michelitsch that I used for the video in Slices of Life - The Shirt. Image (C) TransCoding

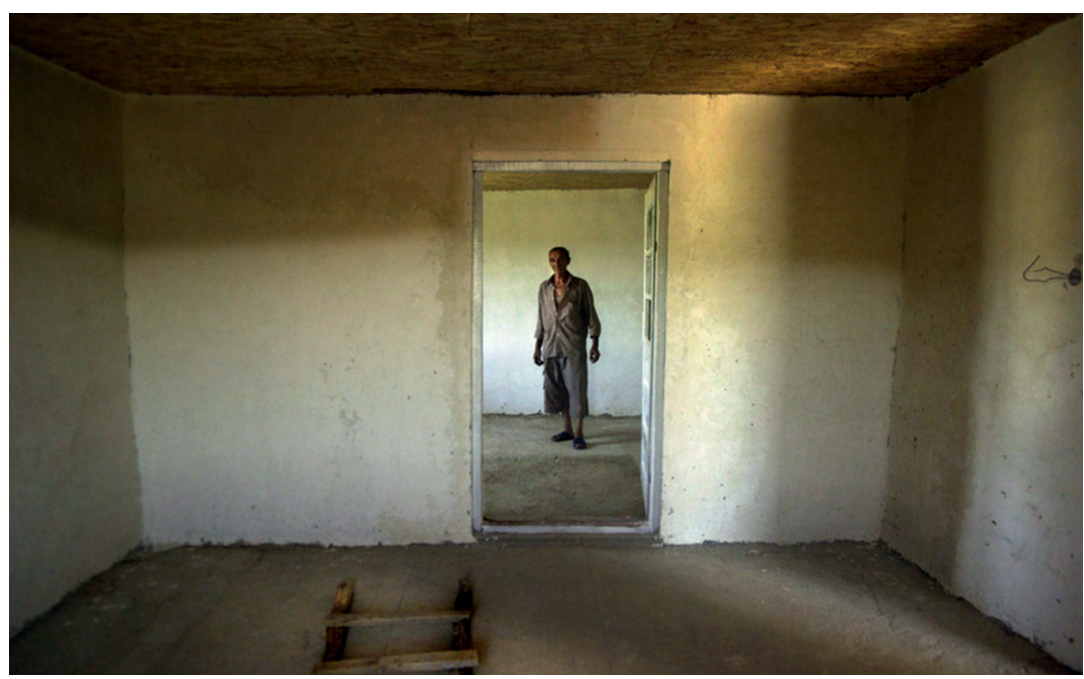

Fig. 1.9. Image of a man in an abandoned house used in Slices of Life. Used by kind permission of Sabina Ulubeanu.

However, each single 'slice of life' - here I am referring to the individual parts that in the end were combined to form the whole piece - each slice consists of a different kind of imagery. For example, I use spontaneous snapshots that were submitted or I visualise texts that were given to me by the community. In one 'slice' on human rights, I incorporate beautiful motifs of Romanian people by 
photographer Sabina Ulubeanu. She is also a community member and gifted the photo series to the project. (Fig. 1.9)

Or take the sound world: I employ sounds which our members have produced under all sorts of conditions - sometimes only with their smartphone, sometimes under studio conditions. Many of the images or sound samples have in common that they show a not overly elaborate, but rather spontaneous aesthetic, like something that has been thrown onto the internet quickly and spontaneously. As a consequence, the often not perfectly produced community contributions influence the overall aesthetics of the artwork. I work with that and try to incorporate the material in an artistic approach that embraces its possible 'imperfectness' in a way that makes sense to all of us and to my concert audience.

This was a challenge in the creative process, but an interesting and rewarding one. To give you an example: let's assume I have a clear visual idea of how I would like to continue in this show and I need portraits of people as video material for the next 'slice of life.' In my mind I have a certain set-up of the person in the image, for instance a close-up with the face in the centre of the photo. Subsequently, I put out a call for entries and ask the community to send me portraits for the artwork. What then happens is that I usually get a kind of portrait that is completely different from what I was imagining. In an extreme case, I might barely be able to recognise the person portrayed in the image, because the photo is taken from very far away, or the background is completely cluttered. Then I would have to work with this material that you could call 'imperfect'- and I'm putting 'imperfect' in quotation marks because the images obviously have meaning and are 'perfect' to the individual community members, or they wouldn't have bothered to send them.

CM: I agree. The exact process that our community members follow is not necessarily planned or formal. I think the informality has been a very interesting aspect. It challenges the authority of Barbara's artistic creation.

BL: To add another thought: In a participatory project like this one, which happens on social media, it is not easy to get people to participate. You have to find something that really interests them and that's doable in a relatively short amount of time, otherwise they won't commit. You have to offer creative topics, which then linger in the back of the mind of our participants. And if by chance they come across an opportunity, they might think: 'OK, this could be something for TransCoding; I'm going to take a photo or make a recording and send it to them,' on the spur of the moment. However, I would like to mention that sometimes people sent us long, elaborate stories and music and pictures. When this happened, I guess we had found something they could really relate to; we had hit a nerve with our call. 
TF: So you think the aesthetics correlate with or react to modes of speaking and writing in social media, in Facebook or Twitter communities? The aesthetic occurs from online practices?

BL: I would say the online practices and their specific conditions of expression certainly play a part in what Slices of Life has become aesthetically. However, the process and the artwork is much more multi-faceted. Take for instance the element of 'story-telling,' which is a prominent feature throughout the artwork. Story-telling comes from popular culture and film. In fact, Slices of Life is a mélange. You will also find traces of classical music, especially with the solo instrument, the classical violin. And because of my background, you get a substantial influence from new music. Slices of Life, the artwork, and TransCoding, the project itself, is a colourful mix, like the people involved. It is a crossover project. We come from many angles, and express our identities in different ways.

\section{Diversity - Crossover}

TF: Let's take one step back. What is the original link between social media and your project? Why did you choose to work via social media with your community?

BL: The social media idea originated from my work with media students in Darmstadt, Germany. In a seminar, we talked about PSY, the Korean pop musician who created the extremely popular song Gangnam Style.

KG: Gangnam Style was a pop phenomenon of large proportions. It was popular in a very 'pure' sense of the word, defying aesthetic 'class thinking.' I found it interesting that people ridiculed and admired it at the same time. This was probably one of the first novelty dances that spoke to a certain type of 'internet humour' often fostered across online media. It was accessible and a little bizarre, it was about body and dance, and about the music, and it lent itself really well to imitation and modification across different contexts. The way I see it, it helped that PSY did not look like your typical glamorous young pop star - quite the opposite. People could relate to that.

BL: I found it fascinating that people across cultural backgrounds would spend so much time with it, use PSY's music, make remixes of it, come together in a group and invent new stories around it. My students loved the song and the hype around it. When we discussed it, the students asked me, "How does a 
song get viral on social media?" and I said, "I don't know," and secretly thought 'I would like to tap into this potential for a contemporary art project.'

Those students, who by the way use social media on a daily basis, were young people that came from popular culture, and as their teacher I was supposed to familiarise them with the new arts. I really liked what my students did, how they used my influence and my knowledge, and melted it into their art. It was inspiring for both sides. Working with these students, I realised that people coming from popular culture and others coming from contemporary arts could join to create a kind of art that is really unconventional and new. My students were the kind of creative people I was looking for in TransCoding. And I knew I could reach them via social media. So this is what I did. I used social media to get in touch with my target audience.

TF: Perhaps one more question about the Ultraschall performance. In my opinion, it was a real success. However, the audience I remember for Ultraschall, for the most part, comes from what one might call 'highbrow' culture. How does this correlate with pop culture?

BL: It's funny that you mention it, because for a long time while working on the artwork for TransCoding I felt a kind of censorship in my head. I found myself thinking 'This is probably fine for the online community but is it also valid for my peer group?' The artwork produced with the community contributions felt almost too concrete, too narrative, certainly not abstract enough for the kind of new music scene I usually move in. Also, if you look at the form of the piece, each 'slice' of the show has a length of something between two and a half and six minutes. That's the length of a pop song. These were all aesthetic choices of mine that were influenced by popular culture and by what I thought my community might respond to, not by new music and my usual peer group. And, there is a lot of story-telling in Slices of Life, a feature that perhaps relates more to popular culture than to 'highbrow' new music audiences.

However, judging from audience reactions, whenever I have performed Slices of Life, my chosen aesthetics have clearly been accepted by my peer group and others. I am very happy about that. My peers really seem to like the work. I guess even intellectuals do appreciate stories and pictures, after all.

\section{Musical Education -}

\section{A Necessary Requirement for Being Creative in This Project?}

TF: This leads us to another topic. On your project website [transcoding.info] you claim that you want to build bridges between popular culture and so-called 'highbrow' art. Theoretically, your community does not need any knowledge of 
classical Western European music theory to participate in the creation of your show. They don't necessarily need to know how to play a musical instrument to be welcomed. To me it seems that you change the culture of composing from one of the individual, highly trained composing 'genius' to composing with a community. Would you please elaborate on that?

BL: Well, first of all, we call our project 'TransCoding - From "Highbrow Art" to participatory culture.' What does that actually mean? For me it meant that I transcoded old cultural practices like playing the violin, composing for classical instruments or performing in the concert hall to practices such as communicating worldwide on the web, using and offering open source freeware in my compositional process, and interconnecting with an online community that could then participate in the creative process. Instead of cultivating the romantic idea of the 'lonely, genius composer,' who works separated from and uninfluenced by society, and lives solely for 'pure' and absolute art, the community and I exchange ideas, thoughts and sounds via blog posts, tweets and Facebook entries. Communication and exchange comes first, before any of us even starts creating art.

And since you mention training and education: When I wrote the music and made the video for Slices of Life, I did what many of our community members do as well. I used everyday tools like the digital audio software on my computer, recorded audio, filmed and took photos with my smartphone, and I searched the internet for sound material. For instance, when I first composed the electronic soundtrack on my computer I often worked with sounds that are available under the Creative Commons license on the World Wide Web. Alternatively, I used samples from the TransCoding SoundCloud channel either provided by our team or contributed by the community. Those sounds were available for all of us and quite a few members of the community utilised them for their own music on their own computer.

It is quite common now that people have digital audio software on their computer and use it as their home studio. You need no classical training for it, but you definitely need to have some experience with the software. In a way, you could probably say creativity has been democratised.

You bring up the violin, and that is an interesting point. As an instrument the violin felt weirdly 'old' to me while composing. Usually I composed everything else first, soundtrack, voice parts and so on, and only later would I come to the point where I thought, 'I have this creative mandate from my research proposal, I need to add the violin.' Then I would incorporate it, and the community was in fact not involved in this particular creative step. I was struggling with the connotations of the classical instrument. In the modern world of social media, it almost felt like a contradiction to use an instrument 
with this kind of cultural 'burden' - or you could also say more positively - with this enormous 'heritage.'

TF: Do you postulate that we should shift our understanding of composing from the isolated genius to collaboration via social media?

BL: No, I wouldn't say so. Not in general nor through social media. However, what is important for me, coming from a background of performance practice, is my relationship with the audience. The audience is often an unknown factor. What are their visions, interests and expectations? This certainly was in the back of my mind when I thought, "Why not communicate directly with my audience through the process of co-creating?' In our case we aimed for worldwide communication, which is the reason for using social media.

CM: I think that social media enables a different form of composing now, which is in addition to all the different kinds we have had before. Looking at popular composition forms today, we for instance find online challenges such as FAWM, the February Album Writing Month. This is an internet challenge, where mostly amateur and semi-professional artists try to write, record and release an entire album during the month of February.

BL: It has a huge attraction to many, mostly non-professional, music lovers and sometimes collaborations are born out of it. At FAWM people talk online with each other and mutually listen to and comment their tracks. It's a tight-knit community. I participated twice in FAWM as a representative of TransCoding, and Clio Montrey took part as an individual artist. We wanted to get the feel of a community different from ours, and to make additional contacts for whatifblog.net.

It would be interesting to hear what Kai says about this topic, because he used to be an active member of a collaborative SoundCloud group. Kai, why don't you tell us about this experimental music group?

KG: Yes, that was before I joined the TransCoding project. When I was doing my own sociological research on noise and experimental music, I was a member of an open collaborative group called Disquiet Junto, which was run by an American media person named Marc Weidenbaum and used SoundCloud as their common platform. Every week Weidenbaum would give people some compositional instructions. He would for instance propose, 'Please compose a piece of music using only this feature,' or 'Use only two tracks and one track should include a particular sound component.' The group members constantly developed creative work off these instructions that came from this one person sitting somewhere in San Francisco. For a certain period of time I think he 
really succeeded in holding together an online community. I would guess he offered people an opportunity to develop ideas that they wouldn't have had if they hadn't joined the community. This might possibly be the core of his project.

CM: I find it important to see these networks as a means of communication. It doesn't negate the fact that we can still compose in any traditional form.

\section{How to Stimulate Creativity}

TF: In the 1960s Robert H. McKim, a Standford engineer, constructed a machine to further the creative potential of people, the so-called Imaginarium. He wanted to stimulate non-linear, visual thinking. The machine had the shape of a dome that one could walk in. It was in fact a multimedia environment equipped with audiovisual features and an air conditioning system that injected scents into the air at regular intervals. People were supposed to enter it to seclude them from the world outside. They became immersed in a rich audio landscape and were shown abstract, colourful films and sounds. They were even encouraged to eat food while inside the Imaginarium. The scientists wanted to make sure that each of the five senses was stimulated. After each test phase the test person had to talk about their experience and provide a narration about what they had perceived. The system was supposed to further creativity and alternative thinking. Incidentally, these were the beginnings of the worldrenowned design department at Stanford. Did you construct anything similar with TransCoding, namely a system to further creativity via social media?

BL: I don't think we constructed anything like a mechanical system or a machine with TransCoding. Our project was about direct communication between human beings.

KG: I agree with Barbara that there was an emphasis on communication. From my perspective, TransCoding went way beyond merely stimulating creativity. The Imaginarium, as I understand you, was about unlocking peoples' creativity in general, whereas the creative actions we asked for in TransCoding were more goal-oriented. In TransCoding communication had to go back and forth, whereas the Imaginarium was a project in which people were prompted to reflect on their experiences within the machine. There is no talking back and forth with a machine, no interaction. 


\section{The Creative Processes Behind TransCoding}

TF: How did the creative process work in the TransCoding community?

BL: I would say it worked in different ways. Our project blog, what-ifblog.net, had a special kind of dynamic. First of all, we had to feed our community with blog posts on a regular basis to keep them interested and engaged. What then really stuck out were the 'calls for entries' - calls for photos, sounds or stories. This is where our community very actively produced contributions, in the form of creative material or small artworks, and shared them among the other members of the community and with us. We on the other hand communicated ideas, incentives or sounds of our own on the blog, on Facebook or SoundCloud, and thus returned the community's investment in us. Everybody could use all the contributions on the basis of a Creative Commons agreement. It was a reciprocal process. We regarded most of our material as open source and people could use it for their own works. At the same time our community members used those calls for entries as incentive for their own creative work. Many of the contributions we got through those call for entries have ended up woven into the artwork Slices of Life.

Logically, these calls for entries only worked if we found a topic that would interest our community. We had to hit a 'sweet spot,' so our members could identify with it. If they then decided to get active and participate creatively, we would feature their contributions on our main blog, what-ifblog.net, on our community blog on Tumblr, on Facebook or on SoundCloud, and we would tweet about it.

The first steps in the creative process were all about the community: we had to think about our community, capture their interest, feature their contributions. Only in a second step did I think about the associated artwork Slices of Life. Only when the material was there could I start working on the actual artwork. And again the community was in the foreground, since I knew I had to respectfully incorporate the community material and had to be authentic to the creator. However, since the material was never the way I had imagined it before in my mind, I constantly needed to adjust my creative work. So from my perspective, the creative process was all very complicated and constantly took surprising turns.

TF: How did you discuss this within the team?

BL: This happened mainly between Clio and myself. 
CM: Usually what happened was that Barbara had an initial impetus and idea for a call for entries in mind and would say, "this is the type of material I am looking for and this is the call that might work for it."

BL: When we designed these calls, they went back and forth between Clio and me. We discussed in detail how we would formulate and express each of them.

CM: My role then tended to be to find creative ways of implementing it online, to develop a distribution plan mainly via Facebook and Twitter, contact people and get them to participate. If we were successful, people would respond with contributions they sent to us. And that material then - I guess it was up to Barbara to decide which parts of it were working for her artwork, and what she would be doing with it.

BL: To give you an example: we had this one call when I needed portraits of people; preferably portraits in which the face was a close-up positioned in the centre of the image. Also, I needed the background to be neutral, not cluttered. The challenge was how to ask for portraits that would come as close as possible to my ideal model. As a solution a former co-worker of ours who was also a teacher at a media school suggested that we offer the community some specific guidelines. These would come in the form of a pixel count, measurements and a frame as to where to centre the person. Since in his experience his students responded better to creative tasks if they had an exact framework, he designed the following schematics to put it in the header of our blog post.

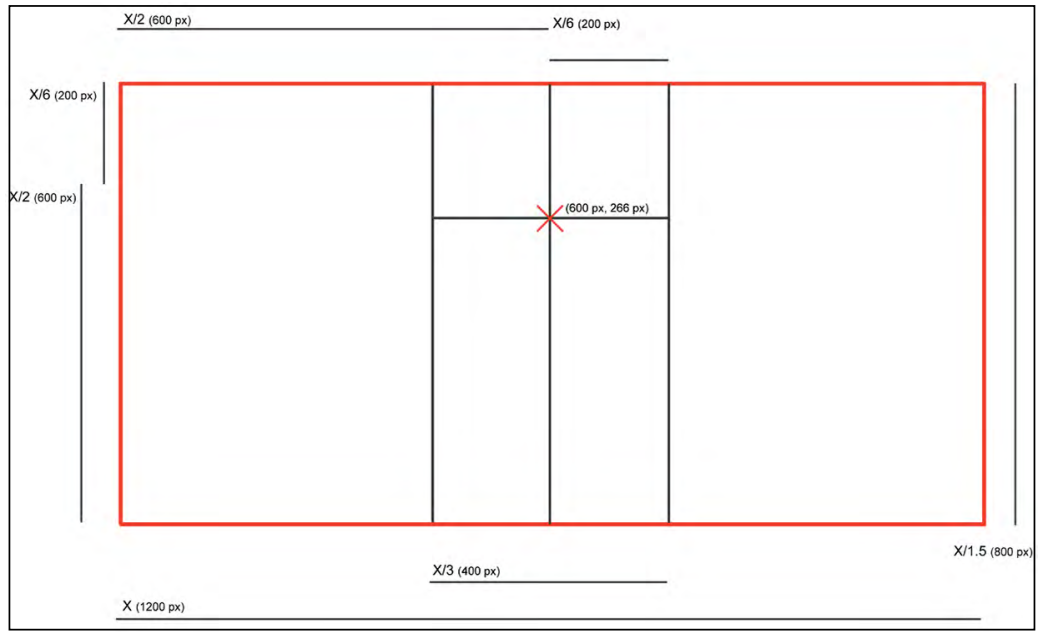

Fig. 1.10: Schematic for the blogpost Call for Entries / Frames of Life. Image (C) TransCoding 
CM: When he suggested this, Barbara and I looked at each other and we thought, 'That will never work, but let's try!'

BL: So we had this blog post with an image - all lines and numbers - and nobody ever reacted to it. It had no relevance to them. Then I got this idea for a new headline: 'Powerful Woman.' It's striking, it triggers your fantasy, it is an important and relevant idea for our community. They know why they should invest time and energy to contribute. And when we asked for images of powerful women, we immediately got a lot of portraits. And that's how we implicated it: We used the new title along with this beautiful photo as a header, which Clio had found (Fig. 1.11). It was a striking image of a vivid and proud looking woman who seemed to have a lot of fun.

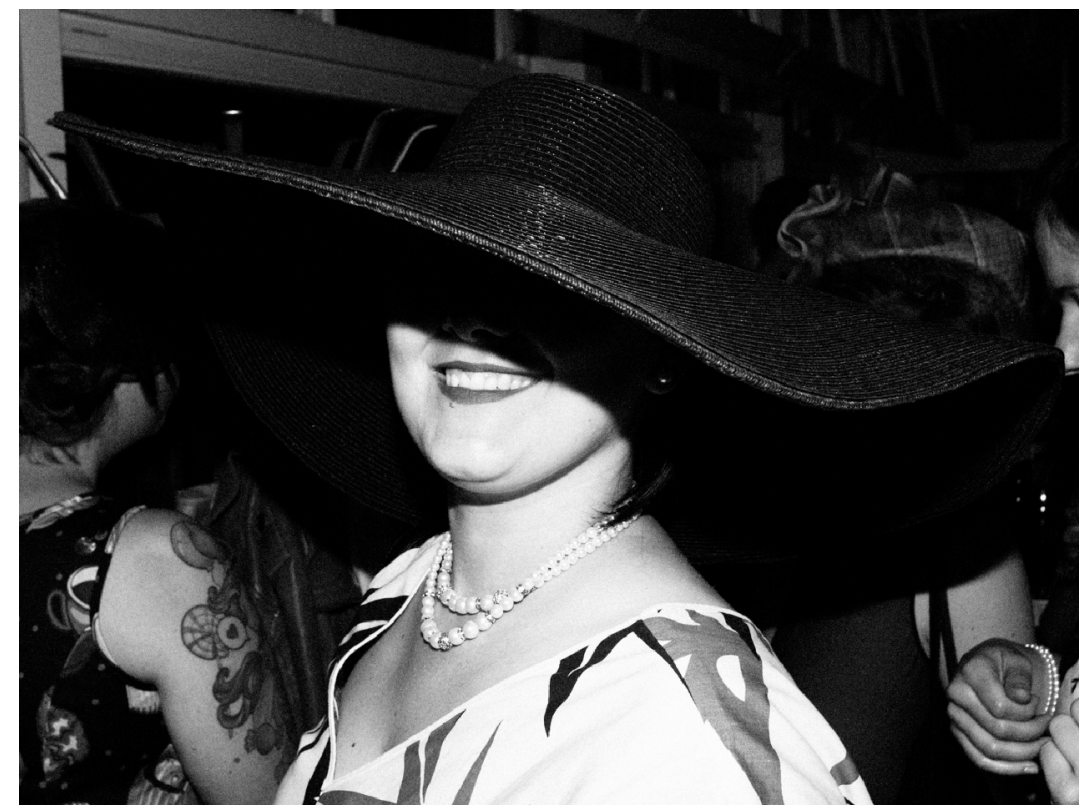

Fig. 1.11: Portrait of a woman from a 'Swing event' in Vienna 2015. Used by kind permission of Alex Dietrich.

CM: The photo is from Alex Dietrich. He's actually quite a well known photographer in Vienna. He was taking these beautiful portraits at a swingdancing event I attended, and that one in particular really struck me. So I asked him if we could use that as an example on the blog to illustrate what we meant with our idea of portraits of powerful women. 
BL: The photo looked joyous, and powerful. However, perhaps the best thing about it was that the face of the person portrayed was half hidden. Therefore, the photo lent itself to projections. Our readers could identify with the charismatic radiance of this person. The image in combination with the new headline was 'catchy.'

CL: By contrast, in the previous call, our co-worker had given participants parameters, such as measurements and placement in the frame. This approach didn't seem to work, but with Alex's photograph, showing worked much better than giving abstract parameters. I think that was a big part of why the 'Powerful Woman' call was so effective; it was very much illustrating all the aspects without having to tell anything.

BL: Also behind the motto 'Powerful Woman' stood an idea, the idea of female empowerment. I assume many women sent in their photo because they could identify with this idea or they wanted to make a statement with their photo.

We received for instance a contribution by a Jordanian, Suad Bushnaq, depicting a Muslim traffic policewoman. On the image there is a lot of everything: traffic, a bridge, trees, photographed through a dirty front windshield. The actual woman takes at the most five percent of the image. You can hardly differentiate her from her environment. This is a typical example of a photo that maybe is not a 'perfect' and artistically worked through image, but for Suad Bushnaq it is a symbol of a female power.

In the end, I couldn't use this particular photo for Slices of Life since the background was too cluttered and the actual motif hardly recognisable. However, we featured it on various of our social media channels.

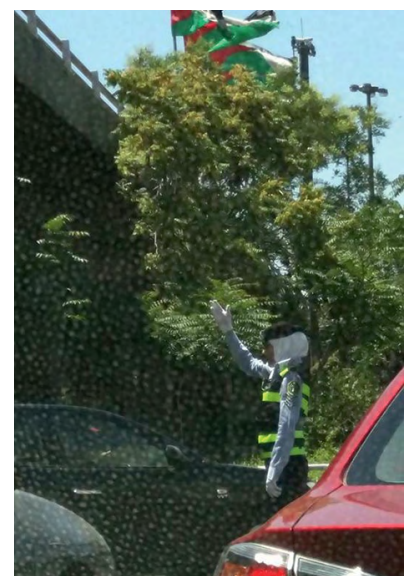

Fig. 1.12: Suad Bushnaq's contribution to the call Powerful Woman. 
We had to relate to the real life of our community members with our calls. We had to find topics that mattered to them. Our calls couldn't be too technical or our community wouldn't respond. We tried to reach our members via captivating headlines, interesting stories, pictures or interesting sounds. I would say this is an important part of how sharing creative processes with the community worked in our case.

\section{Social Media - Self-Promotion or Participation?}

TF: Let's touch on a different topic. Artists often use social media for selfpromoting, as a relevant and important tool for being seen and heard in the market. I think that by doing this, artists massively change values, visibility and the discourse about high art. Your approach to social media is different. You use social media as a tool in the process of creation, which essentially differs from self-promoting. As I understand you, social media seems to be integral to the composing process. Could you describe this a bit more?

BL: Indeed, I see the marketing possibilities that social media offer, but we worked differently with social media. To us they served as a platform enabling us to communicate, to share, to spread content, to interact and to make new contacts. We needed to reach the people on a personal level outside of the music business to build trust and to get them to participate creatively. We refrained from using social media as a means to brand our 'product' or as a personal marketing tool. Clio, as the social media manager, what would you like to add?

CM: If you put something up online and create some noise around it, in the present climate social media tend to be more effective because I think there is this culture of wanting to see and be seen. And Facebook or Twitter effectively feeds into that need. Therefore, I found that using social media was the best way to really make sure people participated in our project. It also worked because on social media you often interact based on some sort of personal connection. Sometimes this connection is virtual only, or small or fleeting, other times it derives from a pre-existing friendship or acquaintance.

KG: We found it necessary to provide an environment where people felt comfortable, where their skills and ideas were appreciated, not merely used or even exploited. From my point of view, a big part of all the work that went into TransCoding was to build and maintain that environment.

BL: I would like to mention that I don't consider Slices of Life the only artwork within the framework of TransCoding. To me, all the social media platforms 
affiliated with TransCoding - for instance the Twitter channel and the Facebook site and of course the main blog itself - make up a participatory artwork which is shaped not only through my co-worker Clio Montrey and myself, but also through the people who got involved. They reacted to posts, commented, shared content, guest-blogged and contributed with their own creative work. The content and appearance of the whole project was formed and designed through this communication, this exchange and interaction with the community. So, the artistic result of the project is more than a 30-minute show called Slices of Life. It is also the participatory art across various social media channels and the audiovisual installation Read me.

\section{The Digital Society}

TF: Theoretically social media empowers people - at least to a certain degree. Virtually everyone with access to the Internet can set up a blog, a Facebook or a Twitter profile to express their opinion. People cast their aesthetic, political or social vote via 'Like' buttons. At the same time, those buttons can be activated by bots or robots, which in my opinion is a dilemma in political practices. We experience in society, and in the artistic and political space, the effects of the digital empowerment of people and then again its cancellation by bots. Did any of this happen in your project and do you consider composing with social media a political activity?

BL: Luckily, bots were at no time part of the culture of TransCoding. I like to assume we avoided such a situation because we always acted according to the Three Rs of Micro-Blogging "Relevance, Respect; and Return” as marketing and communication scholars Kaplan and Haenlein call it. (Kaplan and Haenlein 2011: 110)

What does that mean? Firstly, our blog posts had to be of 'Relevance' for our target group, which means we had to listen to our community before we posted, tweeted or facebooked. It was quite obvious, when we didn't reach them, when a blog post or a call for entry had no relevance for our members. Secondly, we showed 'Respect' to our community members. We were honest in our communication, and people knew they dealt with real human beings. We were transparent about the project and the goals of it, and we consciously used polite, warm and personal language. As for 'Return,' we exhibited every contribution we got on at least one of our social media channels. Several submitters were showcased in portraits or featured via their own guest blogs on what-ifblog.net. Additionally, we highlighted their contributions on our community gallery on Tumblr or on SoundCloud. And when their material was included in the artwork Slices of Life, it was clearly attributed to them. So if they invested in our 
project they got something in return. People entrusted me with their material, and believed that I would find a way to combine their material with my artistic ideas in an aesthetically pleasing and respectful way.

As to your second question, whether I understood composing with social media as a political activity: In my opinion a political aspect lies in the exchange between artist and community and in the permeability of the process. Who has the 'creative authority'? In our project it really goes in both directions. I let people influence my creative process and conversely I hope to empower people in their creativity. The community reacts to my incentives and in the process I am listening and reacting to them. With the audiovisual installation Read me I truly shared the authority over the artwork. People used my software and hardware as a frame which they filled with their own music, text and images. I would say this is the political aspect: We all influenced each other, the community changed my idea of being an artist, we shared authority and I learned from them as they possibly learned from me.

TF: Seen from the perspective of a 'digital society,' would you say that TransCoding is about founding and constructing an internet community with equal rights and access to decision-making? Was access to and participation in the social media channels of TransCoding open to everybody?

BL: This is a topic that necessarily comes up in connection with participatory art. Who is allowed to join in and participate? In theory everybody was, but in reality it depended on people's access to a computer and to the internet and their media competence. China for example didn't appear in our list of countries that visited our blog in a long time and then only sparsely, since in China there are heavy restrictions on social media and internet censorship is strict.

An important point is also that equal rights and equal access could only be granted within the given structures of our interaction, which was shaped by utilising templates of WordPress, Facebook, Twitter, etc. Although these social media sites are very popular among our target group, which is one of the reasons we chose them as communication platforms, they come with constraints. For instance, one needs to be a member of a social media site to be able to participate at all. So members of our community who follow us on Facebook might not necessarily be able to comment on our WordPress site, unless they are or become members of WordPress. Social media templates offer communicational paths such as 'liking,' 'commenting,' 'sharing,' but again they do so with restrictions. Some examples: one cannot include a video in a comment in WordPress; one cannot symbolically 'dislike' a post on Facebook, unless one explicitly 'dislikes' it verbally in a comment. Also, we as owners of a Facebook page and WordPress site were dependent on their algorithms for distribution. Some of our posts may not have been seen, because we didn't use 
words that triggered the right algorithm. Social media platforms impose this kind of limitation.

TF: How did you organise the administration rights for the blog, for the incoming information and for the contributions?

BL: It depended on the platform. In WordPress, for example, I had the fullest administrator rights so I could invite collaborators or guest authors. If somebody was interested in writing a guest post blog, I would give them rights as author.

TF: So that was more by your invitation?

BL: Yes. However, everybody could comment on WordPress and we had an open invitation on the blog to people who would like to write a blog post on our topic of identity [https://what-ifblog.net/your-contribution/]. As for the contributions themselves, we only rarely used our administrative power to say we won't 'allow' or feature this contribution. As far as I remember this happened only twice, when people put something on our SoundCloud group for their own promotional purposes and it had nothing to do with our call at all.

CM: There were a couple of instances where people posted things that just clearly didn't belong. Once for example our community was asked to contribute music based on a drone, using sound samples that we had uploaded. Someone sent a rap track that had absolutely nothing to do with the call and we didn't allow the track on our timeline. In such scenarios there were times where a contribution had to be 'censored,' but mostly people had the genuine intention of participating and seemed to be putting their best effort forward to contribute.

\section{Conduct and Behaviour}

TF: Would you please explain - since that's an important point - what kind of rules you developed?

BL: We didn't.

TF: You didn't? But aren't rules unavoidable? Take for instance current political and legal discussions about hate speech on social media. Here the question is: do we need more legislation acts or do we leave it to the users to become more creative in developing strategies against, for instance, bot-generated hate speech? Or the public discussion about copyrights on texts, videos and pictures. Claims on strengthening copyright laws are juxtaposed by movements such as 
the global Creative Commons network. Was either an issue or of importance for TransCoding?

BL: As I said before, bots were never an issue for TransCoding. People were always very respectful in our community, so we didn't have to set a rule of conduct or code of behaviour. Still, there were rules we set for ourselves. We wanted to always treat our members and their contributions respectfully, and so we valued and commented on each contribution. We tried to quickly thank and respond to contributors, because we knew it is easy to like something, relatively easy to comment, but it's quite a big step to get creative and upload a contribution to a call. Therefore, one of our rules was to openly celebrate each contribution, and to feature them on our main platform what-ifblog.net and on our Tumblr community gallery.
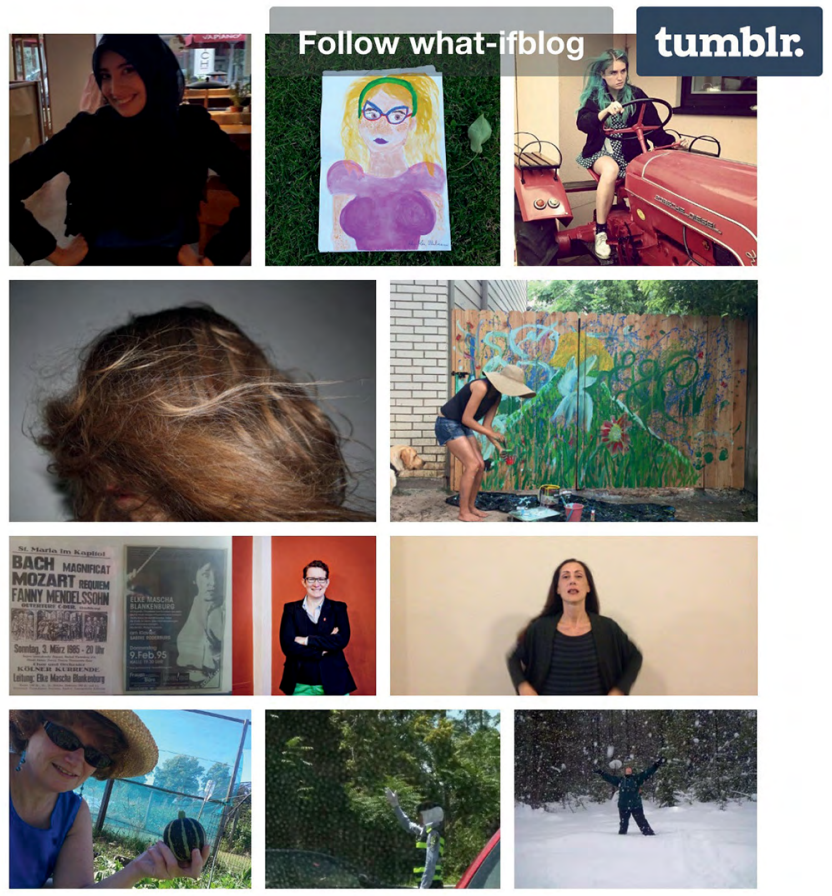

\section{Powerful Woman | \#WhatlfMM | Gallery}

Fig. 1.13: Gallery of contributions to the Call for Entries Powerful Woman featured on the community blog. Image (C) TransCoding 
As for copyright issues, the whole project was based on the idea of Creative Commons, something we made clear on our social media channels, and contributors knew their contributions might be used in the overall artwork. By contributing they declared their consent.

\section{Social Media and Identity}

TF: Internet communities are ruled by more or less obvious frameworks that social media offer. They shape the content and form of participations. Your participants had to fit into these frames. Take for instance Twitter. From a cultural studies perspective, we would say Twitter offers two options of expressing identity: via 140 signs and via hashtags. You could call this a restrictive framework. Tweeting can easily be taken over by bots and those bots could even artificially generate communities. Does that mean social media rules and even generates our identities?

KG: Well, let's have a look at the Chicago School of sociology: they claim that identity is something established in interactions - in a reciprocal process between people and in everyday exchanges. Of course, today social media is a big part of these interactions. Does that mean that social media 'create' our identities? That is an interesting question. I would say that our identities are certainly shaped by both, our use of social media and also by the effects that social media has on our everyday practices. The 'Like' button certainly changed the way we think or feel about content. Twitter makes us come up with little aphorisms that we tend to judge as important or less important depending on the number of retweets. Interactions on public transport are now heavily influenced by the way people look at their phones. The list goes on. However, you refer to the role of technology, namely the algorithms versus the personal input of people. As far as I am aware, sociology has yet to find a clear answer to what the distinctive role of algorithmic processes is regarding all this.

TF: Another question that would interest me. Did people change their identities throughout the project?

BL: This is almost impossible for us to tell, because our members came from all over the world, such as Armenia, the Philippines, Canada, the US, and we had almost no chance to meet any of them personally.

CM: I would be hesitant to say whether community members changed their identities through the project, because in my opinion everyone's personal identity is an evolving entity anyway. From what I noticed in our project, 
though, people seemed quite consistent to their own identity. One of my favourite examples of this is Gloria Guns, who is a human rights lawyer. She was a friend of mine before joining the project and still is. She did a number of different kinds of contributions, ranging from guest blog posts to sound contributions, because she also writes music. She even has one of the Read $m e$ installations. All of these different contributions seem to reflect the same essence of identity. What is very interesting about Gloria specifically is that each time, she contributed fresh, different things, but they all came together to ring very much true and genuine with regard to her own personal and professional identity; and that was across the range of her different contributions. So, although I think personal identities can evolve and change, the participants that I personally observed and had some sort of background information on seemed very true to a consistent identity.

\section{Numbers and Facts}

TF: How many people were involved in TransCoding as a project? Was it a big or a rather small number?

BL: It depends on your perspective. If you compare the number of people to the number a popular food blog might attract, then it is a small number. However, for contemporary music it's quite a big number. We had more than 1,200 followers across our social media platforms. We reached people from approximately 130 countries. In Slices of Life alone we have contributions from Cuba, Canada, France, England, Ireland, USA, Germany, Romania, Bulgaria, New Zealand, Austria, Poland, Russia, the Philippines, Croatia and Armenia. It's spread across four continents. In 2015 - I looked these numbers up - we had 10,000 views on the blog alone. That's not too bad, especially for a niche blog. Moreover, if you compare it to how many people I would have reached in the new music scene through live concerts within the same time span, it is definitely a good number of people.

TF: Perhaps this question to Clio Montrey, the social media manager in the project: Which problems occurred to you?

CM: The real challenges were engaging people across or rather contrary to the technical algorithms of social media sites. It quickly became apparent that Facebook pages, for instance, would be a challenge. Facebook has specific algorithms and considers pages to be commercial; they want you to pay money to boost your post. Since we had decided to run the social media non- 
commercially, without promoting any posts, we needed to use creative methods to make sure that people saw our call for entries.

I would get the word out by sharing our Facebook posts to various groups, sometimes my own page, and making Facebook events, so I could spread the word as effectively as possible and make it feel as personal as possible. I'd send out messages to my contacts that a new call was up and they could participate. Sometimes I would spot a beautiful photo that someone had up on their personal Facebook site and maybe they weren't aware of our call. So I would message the person and say 'hey, did you see our event? Here's the event page, I think your photo would be a perfect fit,' and then they would send it. There was a lot of personal follow-up involved.

In general, Barbara and I both agreed that it would be a good idea to make the interaction more personal, so really that was about nurturing a sense of the community and cultivating openness and creativity. We talked a lot about authenticity during the project. And because we wanted an authentic voice from our community members, we needed to express ourselves authentically too. So, this was what we were aiming for: to be as authentic as possible, and to make sure that everything had direct relevance to our community and to what we wanted to do with the project.

BL: One of the problems I found was that social media are very timeconsuming. If someone were to ask me today if a project like this opens up a new way of composing or if it makes your art well known to the world, I would say 'possibly, but make sure that you really have the time and the drive to do it.' Our community members are real people, not virtual Facebook friends. They don't want to have you in their life just for five minutes. They don't want to be immediately dismissed when they have 'served' your purpose of supporting your composition or research. They want to be seen as real people. It's not anonymous, it's rather personal. This kind of communication needs time and dedication, which was why I asked Clio Montrey to join the team after doing the social media on my own for six months. I realised I couldn't do the arts and the research and the blogging all by myself, and I needed some support in that. The nice side effect of TransCoding is that we now know people from all over the world who we would never have met if we hadn't done this project.

\section{Participative Art Versus Lonely Genius}

TF: This leads me to a question about your personal creative process. You are saying that social media are very time-consuming, and I would imagine you would rather need this time for being creative. Also, with social media you constantly hear voices of other people, and they might stand in contrast to what 
you want, or even be controversial in themselves. However, I would think you needed to concentrate on your own creative voice. So isn't your work with social media the opposite of what an artist needs? What does it do to you and your creative work? Do you feel like you are the artist you want to be, or do you feel like the 'servant' of your community whose task is merely to 'transform' their input? What would you say about that? What makes you creative in this project? Your 'own' voice or the input of others?

CM: I would say that TransCoding hinges critically on using social media. So, since this was my role in the project, I considered the social media not as something that was taking away from my creativity: Instead I was using my creative strategies to help build the project. I'm very proud of the fact that I was able to bring in so many participants. I was able to encourage creative entries from other people and bring their voices into the project. On these voices now rests quite a big part of the artwork. So I think the answer to your question is that it depends on one's personal mentality and on what role you have in this specific project, how you feel about working with social media.

BL: As a composer one could ask questions such as: Why should I collaborate with an online community? How many people can come together and still create a good artwork? Why would I work with people who aren't even professional artists? Isn't my own original creative voice more valid? What about the quality of the contributions? Are they 'good' enough to be included and who decides? These are all important questions that could come up in this context, and there is no easy answer.

However, I am not only a composer. I worked for years as a performer. The relationship with my audience is something that is very important to me. Therefore, the creative process was certainly demanding, but also very interesting. Working with different voices, with contributions that didn't match my expectations but pointed to a new direction in my creative process, was challenging, inspiring, and in my opinion absolutely valid.

TF: There is a long history of writing groups among professionals as well as lay people. Sometimes these groups are politically motivated, sometimes their members look for feedback and inspiration from their peers, or they simply wish to improve their writing skills. If we compare your project to writing groups, what are the differences and similarities?

BL: Well, first of all our communication was not face to face, it happened via virtual channels. This is different from meeting personally and exchanging views as is usually the case with writing groups. Also, our purpose was a different one. None of the reasons you state for forming a writing group applies 
to us. Our project was not in the first place about learning. We invited people into jointly creating a participative artwork. It was not about critical feedback, but rather about offering a platform, and encouragement to be creative, and an opportunity for exchange across cultural domains. Of course, it was also about sharing our passion for contemporary art.

\section{Artistic Research - Ethnographic Sociology}

TF: A question to Kai: You are a sociologist, a researcher and a music journalist. What would you call the most striking outcome regarding your part in the project? What was the most important point for you?

KG: I think the most important point for myself was to get acquainted with the field of artistic research. I was especially interested in the question of how the methodological repertoire of ethnographic sociology could benefit from a dialogue with artistic research and how the two different disciplines might learn from one another. We found many similarities, but also fundamental differences between both domains.

In ethnographic sociology, for instance, many scholars share the opinion that if you immerse yourself in a research field, you should do it with caution and to a limited degree. Accordingly, when applying participant observation as a methodological tool we join our research field, we participate, we write field notes, do interviews, and then we return to our writing desk. We leave behind the world in which we participated in order to reflect, analyse and evaluate. This is different from artistic research. Here artistic researchers are at the same time professional artists, and they continue their careers in the arts, which in turn is the field they are investigating. I found it intriguing to reflect on how the two disciplines can learn from one another in order to expand their methodological palette.

BL: As artistic researchers, we do our work within our peer group, which can be ethically and artistically challenging. Moreover, we need to make sure to reflect as objectively as possible. We also might want to be daring in our art, and maybe widen the boundaries of the field; this can be tricky, because you never know what's going to happen if you do so, and how people (including our peer group) will react, but so far it has been a rewarding experience.

TF: Thank you very much. 


\section{From lonely GeniUS to COMMUNity Creation - Whose Volce Matters?}

In the second part of this monograph, I explain the rationale for the design of our participatory arts project and discuss the theoretical foundations that underlie its formation while introducing the kind of strategies we needed to apply to foster and unlock the creativity of the community. Additionally, I investigate whether the project led to a fruitful exchange between the TransCoding team and our community that added meaning to both, and how the role of the artist changed through the project.

I start with a definition of participatory culture in the context of TransCoding, followed by thoughts on the social media and content strategies applied (chapters 4 and 5). I look at the actual art production (6) and search for the motivation of contributors who decided to participate in the creative endeavour (7) and next, I feature the community's voice by presenting contributions from community members that shaped one of the main artworks of TransCoding, Slices of Life (8). Last, I investigate the distribution of authority in the creation process within the ecosystem of TransCoding, which consists of the online community and the artist(s) involved. I present a key moment that changed the course of the project (9) and introduce in detail the work with single community members on the audiovisual installation Read me.

I use as my theoretical framework the Self-Determination Theory (SDT) of Edward Deci and Richard Ryan, scholars for clinical and social sciences in psychology and education, and the theories on participatory culture via web 2.0 of researchers and cultural scholars such as Claire Bishop, Henry Jenkins, Nina Simon and David Gauntlett. In order to arrive at a definition of participatory culture relevant to our project and to locate our position within this concept, I will first address what such a culture is, and which specific model we developed for TransCoding. 


\section{ThEORY OF PARTICIPATORY CULtURE}

In their paper Confronting the Challenges of Participatory Culture - Media Education for the $21^{\text {st }}$ century, Henry Jenkins and his co-authors Ravi Purushotma, Margaret Weigel, Katie Clinton and Alice J. Robison define participatory culture as

"a culture with relatively low barriers to artistic expression and civic engagement, strong support for creating and sharing creations, and some type of informal mentorship whereby experienced participants pass along knowledge to novices. In a participatory culture, members also believe their contributions matter and feel some degree of social connection with one another (at the least, members care about others' opinions of what they have created)." (Jenkins, Purushotma, et al., Confronting the Challenges of Participatory Culture - Media Education for the 21 st century 2009, xii)

They state that forms of participatory culture include "affiliations," memberships that can be formal and informal, and in online communities are centred around various forms of media. They embrace "expressions" by producing new creative forms), "collaborative problem-solving" by working together in formal and informal teams to complete tasks and develop new knowledge, and "circulations" - that is, they shape the flow of media through podcasting, blogging or micro-blogging. (Jenkins, Purushotma, et al., Confronting the Challenges of Participatory Culture - Media Education for the 21st century 2009, xii)

Where can we find those characteristics of participatory culture in the set-up of this project? For TransCoding I chose social media as a means to address our community, since our target group used them as a common tool of communication, so that there was a relatively low barrier to self-expression. I established the topic 'identity' as the focus of the artwork and our social media presence, since I assumed that almost everybody will deal with the notion of his or her identity at some point in their life. The comparatively universal topic served as an easy way to enter into our project, and provided a low threshold to participation. Affiliation and online membership of TransCoding could be obtained through becoming a reader or follower of the associated blog whatifblog.net, through observing or actively participating in our communities on Facebook and Twitter (or any of the linked social media channels), or through personal contact with one of the team members.

On our social media channels, my colleague Montrey and I supported the community's engagement through a variety of actions. Our posts, related to our topic 'identity,' gave opportunity to reflect on and to work creatively with what we offered. As inspiration, and to encourage the sharing of knowledge, we 
featured artworks from the world of contemporary art that were related to our topic. Additionally, we offered our followers incentives to express themselves and get creative by posing smaller or bigger artistic challenges, and by giving away free sound samples via a Creative Commons licence on our SoundCloud channel. We also actively encouraged members of our community to write guest blogs, and generally furthered communication and exchange as a means to support the feeling of affiliation.

During the production of the main artworks I worked directly and collaboratively with single community members for the audiovisual installation Read me (described in depth in chapter 9, 'A Potential for Change'). We worked through collaborative problem-solving. For Read me the participants could actively decide about the musical, visual and text content for their personalised version of the installation and could produce the content entirely by themselves, if they chose to do so. In comparison, the collaboration in Slices of Life was largely more indirect, although the community exerted a strong influence on that too and provided content in the form of texts, images and sound.

Last but not least, our members and their work mattered to us. We shared and showcased their creative work and contributions via our main blog whatifblog.net, the companion blog on Tumblr (what-ifblog.tumblr.com), a blog that explicitly served as a community gallery, on Facebook, on Twitter, on our SoundCloud channel, and on Pinterest and Instagram. Circulation was also supported through our members via their own social media channels.

Thus, the work we did within the framework of TransCoding was based on a low barrier to artistic expression, support for the creation and sharing of creations, affiliation and membership, collaborative problem-solving and the circulation of content produced within the project; in short, the principles of participatory culture specified by Jenkins et al..

Next I would like to look at the democratic affordances the project offered its community members.

\section{Access and Authority}

Media scholar Münker states that a participatory public "wishes for interdisciplinary discourse, enables the development of new concepts, consciously exceeds familiar organization and invites the observer to look for new perspectives and paths" (Jank 2012, [translation from German to English by the author]). Referring to philosopher and sociologist Habermas, he claims that there are four criteria for a sphere of civil openness: "Access to it is principally open, the members are completely equal, the choice of topic is entirely open 
and the circle of potential participants is open" [translation from German to English by the author]. (Münker 2009: 36 f.)

The project TransCoding was theoretically open to anyone who had access to uncensored internet and a computer. The spread of the project was wide. In the year 2015 we reached visitors from 19 countries with almost 10000 views on the blog alone (Fig 2.1).

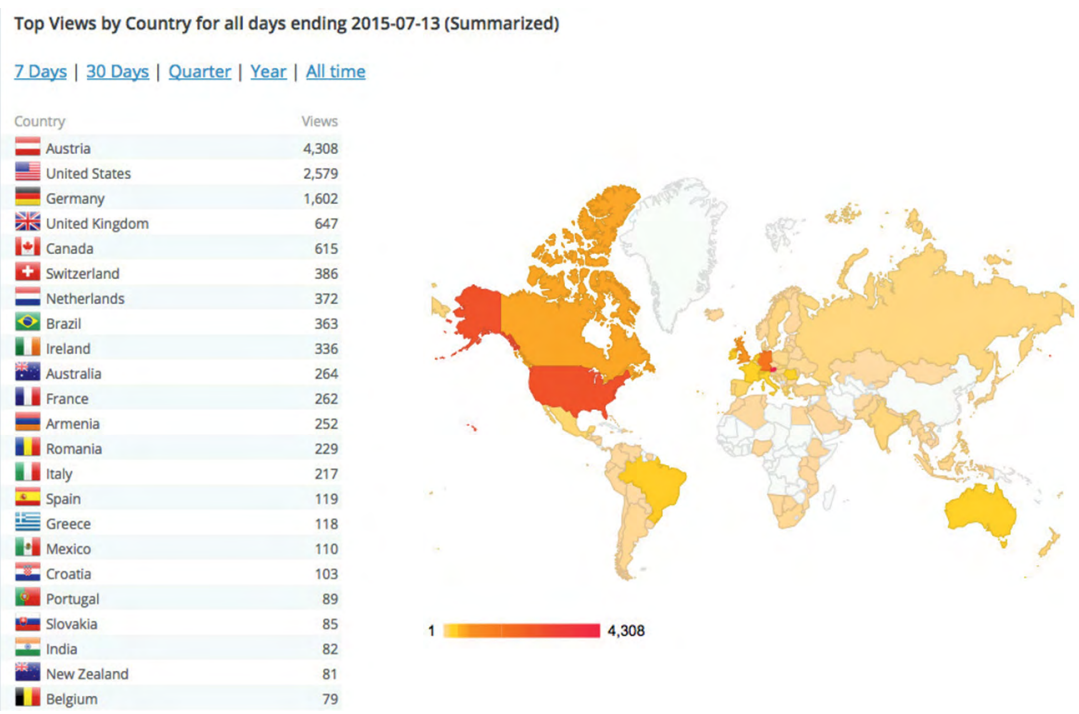

Fig. 2.1: Statistics of the blog what-ifblog.net on 2015-07-12. Image (C) TransCoding

In contrast to Münker, museum consultant and scholar Sabine Jank talks about the limitations of participation when she addresses participation as a utopian idea, and discusses the feasibility of participation in her article Strategien der Partizipation [Strategies of Participation]." (Jank 2012) She states that a "participatory public" is generally seen as a utopian idea, because its multiple perspectives and congenial composition are linked with a breakup of the traditional production of knowledge. In her opinion the media scholar Stefan Münker, whom she quotes, describes an ideal type of participatory public.

Our core target group was an internet-literate young audience of digital natives. Its members were interested in creatively expressing themselves, came from popular culture, and wouldn't necessarily attend classical contemporary multimedia performances. They were what arts marketing expert Keith Diggle describes as being an apparently 'unavailable audience'; non-traditional attenders at an organisation's concert who may nevertheless be reached in new ways. (Diggle, 1994: 32, cited by Carboni 2011). Contributions from participants outside the target group were equally welcome. Although we explicitly addressed a young and tech-savvy audience, I cannot deny that there 
were possible impediments for participating in TransCoding, starting with the problem that people might have restricted access to the internet, or limited or no access to technical hardware such as computers, recording tools, photo or video cameras. Other constraints to participation included a general lack of technical knowledge in handling either features of social media (uploading blogposts on WordPress) or using software tools such as digital audio workstations. However, we tried to regularly offer calls for submissions that didn't ask for technical expertise. Last but not least, language was an issue. Although we tried to open the project as wide as we could by using English, not everybody is comfortable with posting in a language other than his or her mother tongue.

The key features of web 2.0 allow for free classification and arrangement of information in the form of social tagging (the so-called folksonomy), user participation in the form of crowdsourcing, and contribution through evaluation, review and commenting. Users are empowered to shape their medial reality (7 key features of web 2.0 ( webAppRater 2010). Our content was made available to share, reuse, distribute and edit as a basic sign of authority and autonomy. A wide dissemination and dispersion allowed for content delivery via multiple channels, including file sharing and permalinks.

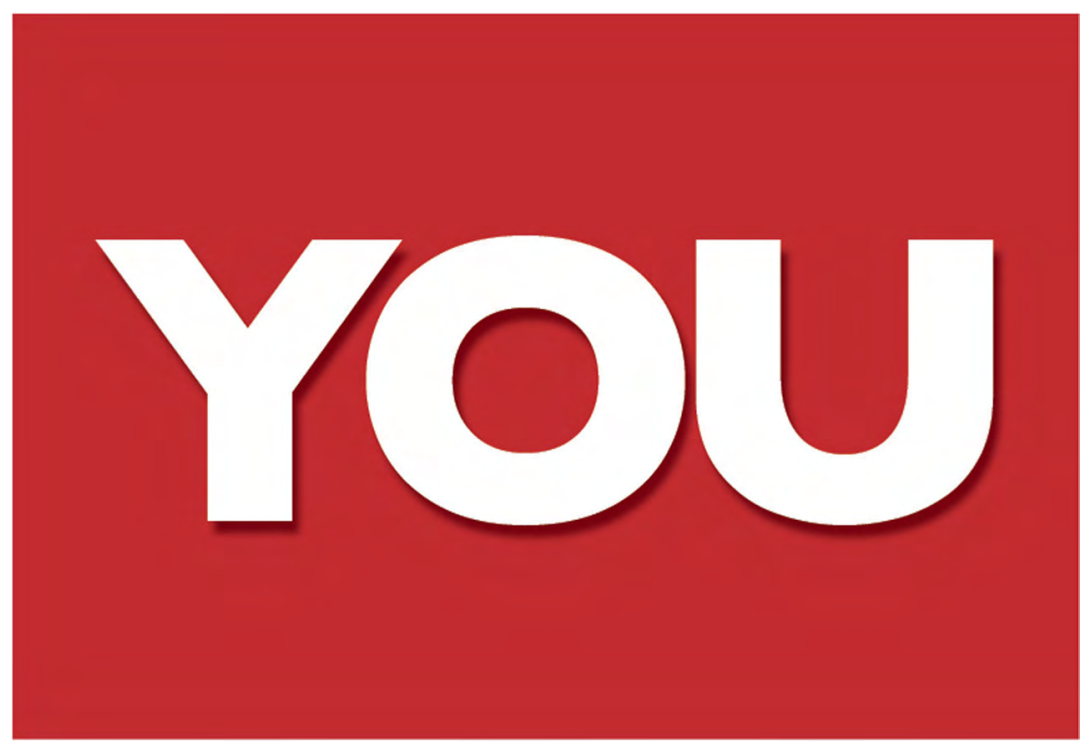

Fig. 2.2: "In 2006, the World Wide Web became a tool for bringing together the small contributions of millions of people and making them matter." (Lev Grossman Monday, Dec. 25, 2006, http://ti.me/18GMbym, visited on 2016-2-29). To TransCoding the 'You,' the community, matters. Image (C) TransCoding 
Jank argues that another potentiality of participation is the conscious establishment of a "philosophy of conflict" that understands critical debate, protest and provocation as a micro-political practice through which all participants become active agents thus constituting a public that is equal. This raises questions of power constellations, objective knowledge (and access to it), unrestricted communication, open, critical dialogue and the dissolution of traditional practices, as well as public access and the congenial inclusion of outsiders. Participation here is characterised by a processual quality. (Jank 2012: 147) These questions have become important in our project, as I will elaborate later in chapter 9 ('A Potential for Change: Case study What if we had wings? - An Area of Conflict').

As the lead artist in the project I opened up the development of the associated artwork to the influence of the community, from the conceptualising phase onwards. The dialogue with the community came first, before concrete ideas for the actual parts of the artwork Slices of Life came to life. This was not always the case, but happened as the result of a conflict in the initial phase of the project, when the then still acting art collective of TransCoding first conceptualised and composed a substantial part of what was to be the main artwork without any interaction with the community, and invited the community only much later to contribute. Our participants were solicited to contribute texts that we integrated into the video for our artwork What if we had wings? for violin, (live) electronics and video. However, the kind of material and the multitude of diverse contributions the community presented to us following our call for entries provoked pronounced discontent among the artists and led to a substantial dispute about the sense of community participation in general and participation via web $2.0 \mathrm{in}$ particular. The conflict led to the dissolution of the collective, and I continued as the single artist working in interaction with the community, supported by the research team only.

Subsequently, I progressively changed the model of participation from 'contributory,' where participants "are solicited to provide limited and specified actions or ideas to an otherwise institutionally [i.e. by myself] controlled process" (Simon 2010: 187) to 'collaborative' leaning towards 'co-creative' participation. According to Simon, "[i]n collaborative projects, visitors are invited to serve as active partners in the creation of institutional projects that are originated and ultimately controlled by the institution," whereas "ii]n co-creative projects, community members work together with institutional staff members from the beginning to define the project's goals and to generate the program or exhibit based on community." How did interaction with the community actually shape the specific topics and aesthetics of the artwork Slices of Life?

As a first step, we provided context on the topic of 'identity' in general and in the arts in particular on our project blog what-ifblog.net and extended it across 
our various social media channels, thus establishing an initial community. We also initiated discussions on our topics on our project Facebook page and our personal Facebook sites. Community members could make their interest and preferences clear through liking, commenting, or (passively) ignoring our posts. This process demanded continuous evaluation and (re-)design of our system according to the reactions or non-reactions of our community while being aware of our research objectives.

Later, we started our 'calls for entries.' Here, community members could actively participate in the form of contributing photographs, text fragments, or presenting us with sound samples to compose with. They could shape the overall narrative through their own story-telling, influence the musical material by introducing me to their own compositions via challenges on our SoundCloud group, and have an effect on the visual aspect of the artwork by submitting photos as response to our calls for entries.

In a third step, a selection of the community's stories, sounds, compositional material and photographs found their way into several smaller and bigger artworks such as the show Slices of Life $(2016 / 17)$ for violin, soundtrack and video. As in the overall project, the topic of Slices of Life was 'identity' and the many facets identity can take on. Images, texts and sounds for the show are in large part contributions from the TransCoding community, from contributors in Cuba, Spain, Canada, Croatia, USA, Austria, Philippines, Armenia, Russia, England, Poland, Bulgaria, New Zeeland, Germany, Romania and France. In TransCoding's second main artwork, the audiovisual installation Read me, I took a third and even more radical step. Here, participants had the option to shape and personalise the visual, textual and musical content of the installation to their taste, using the hardware and software framework I provided (more on that in chapter 9, 'A Potential for Change'). In a fourth step, the community used material we provided to make their own art, thus gaining sole authority over their creative decisions.

In conclusion, I can say that free access to the project, influence on the discourse and shared authority were the democratic affordances the community was offered. We consciously worked on the idea of defining the (commonly hierarchic) relationship between artist and community as one of permeability and mutual influence.

Before I present selected community contributions in chapter 8, I would like to investigate what kind of incentives trigger the engagement of community members, and what it is that motivates participants to invest in a call for entries and our project in general. 


\section{Social Media - Content Strategy}

When I started the project, sceptical onlookers asked why anyone would ever contribute to my artwork. I - a privileged white woman from Europe who even got paid for her work on the project - was putting together a show for violin, electronics and video and asked people to participate in the creation of it. Why should anybody feel motivated to do so?

My task in conceptualising the social media and the artistic approach was moreover guided by an explicit research question: How could I further crossover between high art and popular art by offering creative and intellectual incentives to the community for participation, while on the one hand staying true to the domain I came from, namely contemporary art music and multimedia art, and on the other hand listening back to and channelling the community's own creative voices. Confronting the challenge of motivation in participatory culture, and in particular in this project, was therefore central to the progress of the work and concerned especially the social media content strategy.

One can identify at least three dimensions of TransCoding that were important in terms of the interaction between artist and community and the involvement of single followers: the social, the content and the artistic dimension. Within the project set-up they were emphasised through different means and could be experienced in both the communicative and the creative process. The three dimensions need not to be seen in isolation but in relation to each other. Their interplay determined if and how people interacted, and it influenced their motivation for participation and the manner of their involvement. However, they weren't necessarily equally relevant for the individual participants at all times.

The social dimension played an important role in opening up the project to a wide audience, establishing a feeling of a close-knit community. Furthermore, it was essential in bringing down possible barriers of communication caused by a traditional and hierarchical understanding of the role of the artist, a position that gives the sole aesthetic and decision-making authority to the 'creative genius.'

How did we structure social interaction? First of all, we understood the project as an inclusive one, meaning that we opened it up to people of all countries, races, religions and ages. We openly and clearly invited people to interact and contribute on our blog what-ifblog.net. Within the project, interactions, communication and creative outcomes were regulated by a social agreement that clarified the possible ways to contribute and to join the project via guest blogs, and the creative and legal processes behind it (https://what-ifblog.net/ about/, https://what-ifblog.net/your-contribution/, https://what-ifblog.net/ disclaimer/, https://what-ifblog.net/2014/04/18/you-and-us/). We explicitly 
and repeatedly invited people to participate and make their voices heard, and we guaranteed authentic appreciation of community contributions. We strove for truthful and authentic communication between professional artist and community on equal footing. We engaged in personal contact and exchange via chats and email, if this was asked for by individual community members.

The design of the blog - warm and colourful, not overly sophisticated yet visually appealing, with an emphasis on expressive photos as thematic hooks - reinforced our effort to make 'highbrow art' accessible. We downscaled the seemingly 'elitist' and exclusive process of making art by giving insight into the work of a professional artist. Under the heading 'Making of,' for instance, I offered insights into the deliberations and preparations at the start of our creative project; discussed the development of an artistic concept in general; allowed our audience to share in our joys and doubts on how we worked when producing Slices of Life; announced concerts, at which I presented the jointly produced artwork; and later shared the associated (video or audio) documentation. The team's social media manager and I worked on turning the image of the mystical creative 'genius' into that of a person who is involved in the same process of preparation, incubation, insight and evaluation, of success and failure as every other ordinary person in any creative process.

We worked on managing and creating a sense of a creative community. At regular intervals we called for community contributions to gather material for the show via our social media channels. We observed two kinds of participation mode: passive participants read our posts as 'silent' followers of our various channels, or liked individual posts, sounds or images we presented; the active ones became engaged in different activities in the framework of the project.

We came up with a list of possible forms of participation, which we graded from the least to the most challenging:

1. Comment on a (blog) post, especially on what-ifblog.net, on the official Facebook page or the team's personal Facebook sites, or on TransCoding's Twitter channel, or on any of the visual or audio social media channels of the project.

2. Supply a link within the comment section of our WordPress, Facebook, Twitter or Tumblr channels.

3. Contribute with text on WordPress, Facebook, Tumblr or Twitter.

4. Pingback on our blog via contributor's blog, a feature that was only possible for subscribers to WordPress.

5. Upload a photo on Tumblr, Facebook or Instagram and share it with the TransCoding community.

6. Film a video and share it with the community on one of the associated social media channels. 
7. The contributor creates their own piece of music and shares it via SoundCloud with TransCoding's community.

8. The contributor remixes sounds from the project's SoundCloud with their own music, shares it via any social media channel and links it to the project.

9. Download a mute video offered by us, score the music, re-upload on YouTube and share it with the community.

At the heart of our propositions for participations stood the 'calls for entries' on what-ifblog.net that in turns asked for image, sound or text submissions. Sometimes we also encouraged our followers to tell us about their personal context and creative disposition. Usually a call was embedded in a larger chain of thematically linked blog posts or events. We let community members decide how to approach the calls and which agency to follow: some got creative for themselves because the call had aroused their interest; others entered an intersubjective exchange with us and consciously contributed to the multimedia show. (This will be described in detail in the following chapters). We explicitly featured the community's creative involvement and fostered their sense of accomplishment by dedicating a full category to them at what-ifblog.net under the header 'You, Us and the Project' and by offering a separate blog on Tumblr for the exhibition of their works.

The content dimension was central in directing general attention to the project, piquing people's curiosity and making them return to the blog. By choosing 'identity' as a topic that invites exploration, touches emotionally and is part of everybody's existence, we opened the access gates wide for everybody on a macro level. 'Identity' can be considered from different perspectives and we tried to cover many. We started from Greek Antiquity, included customs (and religions) from New Zeeland, Cuba, Mexico, Morocco; entered into the fictive lives of personalities our participants had invented; featured individual living artists with their take on identity; touched repeatedly on (undoing) gender; caught frames of life through the lens of our photographers; inquired how sound could relate to our community's sense of identity; made the notion of human rights and diversity part of our discourse on identity; and debated current topics such as virtual reality or coding and their impact on our lives.

Specifically related to our research claim of promoting classical contemporary music and multimedia art, 'identity' was as often as possible covered in direct relation to artworks. We looked for art that had 'identity' as a topic, interviewed artists of different genres on their work and creative processes to discover their creative identity, or introduced our community to festivals and events that featured new art to draw their attention directly to the field. The category under which we featured these blog posts was called 'Art We Love.' We wanted people to feel our enthusiasm about the art we promoted and 
strove to give our blog posts a personal touch. This stood in direct relation to the social dimension we aimed to establish.

'Art We Love' additionally served us as backdrop for the creative challenges we set out for our community. Those were designed to be entertaining and interesting to explore, trigger different kinds of motivation and offer value in themselves for the participants, while fitting into the context of the artwork I developed in the framework of TransCoding.

\section{'Calls for Entries' on what-ifblog.net}

These were the four early calls for submissions that we featured on what-ifblog.net in which we asked for different kinds of contributions that could take the form of music, texts, video scorings or photography. I will introduce you to the texts and add a selection of responses.

Window shopping for a new identity. Vienna. Spring 2014 (April 15, 2014) was the very first call on what-ifblog.net, six weeks after I had launched the website, and it aimed to stimulate (artistic) comments from our participants on our blog. At the same time, I was curious whether and how community members would artistically express their concept of identity and freedom. Both comments we received featured music.

"If you find a picture/quote/music related to identity or freedom of choice in your home town, in your image/sound/or text-archive, on the internet, or in your creative imagination, please let us know and share it with us." (Lüneburg, Window shopping for a new identity. Vienna. Spring 20142014 )

Response by Sylvia Hinz, Berlin:

"thank you for this great project and the initiative! ... i'd like to share with you my 'windserie' ... enjoy! ...

identity ... my 'windserie' describes my preoccupation with the topic wind and breath - all my life. i grew up near the coast, always loved the sounds of wind and storm, started to play the recorder aged 7 and have a focus on breath (including breathing sounds) and wind noises ever since then. and ongoing ... this is my identity. http://sylviahinz.bandcamp.com/album/windserie" (Comment by Sylvia Hinz on the blog post: Lüneburg, Window shopping for a new identity. Vienna. Spring 20I4 20I4) 
The call City Hopping for a New Identity - the Sky is No Limit (April 24, 2014) asked for life stories of community members expressed in the format of a photo, text or video.

"What if we had wings? What if we could escape our personal boundaries, follow our visions and dare the impossible?

Have you ever moved to a different country? Did you go on an adventure tour at some point in your life? Has there been one place of which you can say that having been there has changed your perspective on life? Did you ever have to learn a new language or you would not have been able to communicate with the people around you? How was it like? How did you feel? We would like to know what shaped your life. What shaped your identity as it is now? $\cdots$

Send us a photo, a text, a video of a place you visited, an impression of a country that shaped you, a recording of a language you miss. Pingback it to us by adding a link to this post or add a comment. We are looking forward to hearing from you." (Lüneburg, City Hopping for a New Identity - the Sky is No Limit 2014)

It triggered several responses, among which the guest blog Armenia-Homeland by Anahit Mughnetsyan was the most elaborate (documented in chapter 8, 'The Community's Voice'). The topic of spirituality as an attribute of one's identity became established through Anahit's text.

The challenge Who is Damian, Lia and Barbara...? (May 15, 2014), which we launched on YouTube, was quite ambitious. It demanded not only artistic skills but at the same time technical knowledge to score the videos and handle the downloads and uploads on YouTube. Also, participants needed to have their own YouTube channel to be able to participate.

"Today, we proudly present the new YouTube channel of what-ifblog.net. On this channel we will feature art works that have been sent to us in reference to our project. Enjoy exploring.

Our first idea was to start the remix challenge: Who is...? We had a fun evening videotaping little sequences of the artist team of what if? in our spooky Viennese basement. We then composed individual mini-video portraits of each of us, and uploaded them on our channel for you to download and play around with. The idea is that the music you add will change the identity of the person on screen.

If you would like to take part in this arts project follow the link to our channel bit.ly/1jKOIuD, download one of the artists' videos and compose the music for it: Who is Damian/Lia/ Barbara...? Think about a musical character you would like to give him/her. Find a musical identity you consider fitting. 
Become a part of the project. Compose a piece of music, upload the video with the music on YouTube and send us the link (if you like as a comment below), so we can feature it. Be creative, have fun, we are looking forward to your artwork!"

(Lüneburg, Who is Damian, Lia and Barbara...? A Remix Challenge on YouTube 2014)

Several young sound artists responded to the call. They fell squarely into our target group of tech-savvy social media users, who came from pop culture but were inspired by the challenge.

Link to the scorings of the video Lia by Christian Schwind and Marco Pietrolungo http://transcoding.info/english/book.html - PART II CHAPTER 5

Call for Entries: Creativity is Contagious was inspired by a quote from Albert Einstein which I had stumbled upon on the internet. It was the first call in which we asked for photos. Additionally, we introduced a new feature of the project, the community gallery on Tumblr.

"We're intrigued by the question of where creativity comes from. We do believe that one creative act or work has a tendency to inspire others. Thus, we'd like you to take part in our next call for entries, which is an experiment to see just how far the seed of our creative call will spread.

We all know the concept of a selfie. But what about a shelfie? Urban Dictionary defines a shelfie as 'A picture or portrait of your bookshelf. Showcasing literature IN ALL ITS GLORY! ... Not to be confused with selfie.' Literature is one way to do it. But as we have seen, creativity comes in many forms.

\section{Instructions:}

Submit a shelfie that fits within our broader definition: anything that expresses your creative identity, on a bookshelf. It can be your favourite books, of course. But it can also be pens, paper, notebooks, pencils, instruments, decorative items, artwork or clothing. ... Selected entries will be featured on our What if? Community Gallery on Tumblr ..." (Montrey, Creativity is Contagious, Pass it On 2014)

Responses included among many others Maria Baez Vales' beautiful harvest picture. 


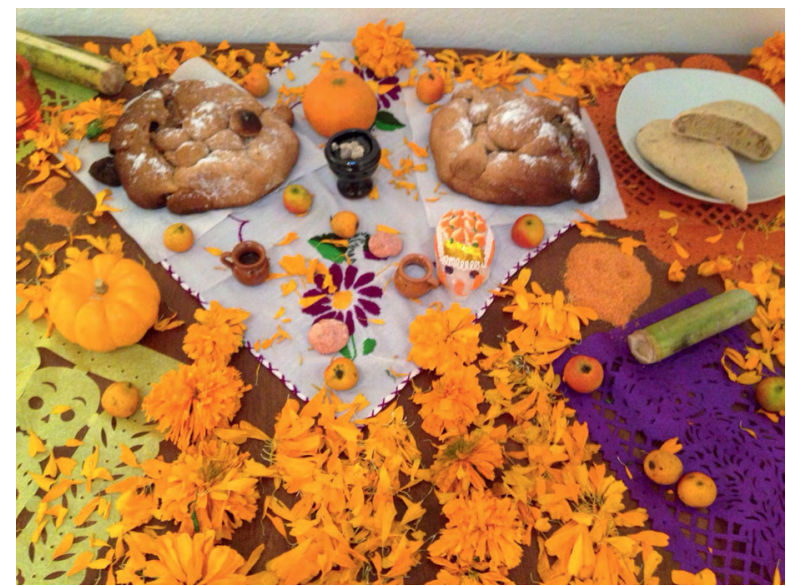

Fig. 2.3: Maria Baez Vales' (Mexico) contribution to our call for shelfies.

Gloria Guns sent us her greenhouse shelf along with an explanation: "I am based in Cambridge Bay, Nunavut, Canada, which is very far north in the high Canadian Arctic. Here is my submission for the call for shelfie entries. It's my vegetable garden, which I keep on a low shelf, obviously indoors because the Arctic is always cold. I am a musician and a writer, but lately I have been really enjoying tending to my garden, as I find it incredibly therapeutic - and fresh produce is hard to come by in the Arctic (and expensive)." (Gloria Guns on her submission on Tumblr).

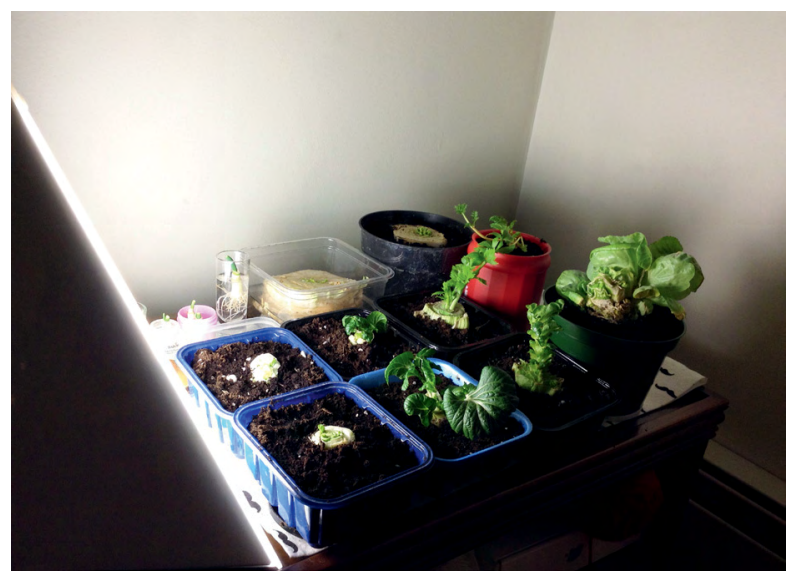

Fig 2.4: Gloria Guns' greenhouse shelfie. 
In the next chapter, I will trace the search, the ruptures and the continuities in the development of the artwork Slices of Life that occurred because of the unusual way of creating in interaction with the online community. 


\section{The ARt Production}

\section{Preparation, Incubation, Insight and Evaluation}

Creative processes go through the phases of preparation, incubation, insight and evaluation. (Wallas 1926) During the development of Slices of Life, those phases were shaped by the interests, participation and general influence of the community, and needed constant adjustment.

Within the preparation phase, I searched for and presented topics that on the one hand served the exploration of the general theme 'identity' and the communication flow on our social media channels, and on the other hand advanced the creative work on Slices of Life. The process was iterative and was characterised by a constant probing for areas of interest that might trigger the participation of our highly heterogeneous community. Content needed to be carefully prepared for people who we wanted to lure into the project, especially when posts were concerned with contemporary (classical) art music or new multimedia art. We needed to find an angle that would talk to our community of mostly non-professional creatives that had little or no experience with this kind of art form.

How we proceeded from there in the form of our calls for entries depended on the reaction and interest of these people, who came from different cultural backgrounds, most of whom we had never met, and who didn't belong to our peer group. The responses to our calls and the material that came in, in the form of photos, drawings, texts, sounds or videos were usually quite different from what I had in mind when I conceptualised the calls. The community interpreted them in a way that met their personal interest, not necessarily the demands of the growing artwork. In fact, the overall content of Slices of Life was grounded in democratic affordances that we granted the community. Our members influenced the emergent fields of interest by passively rejecting or actively reacting to what we presented, and they shaped the content by way of their submitted narratives, imagery and sounds. There was a constant need for thematic adjustments and aesthetic fine-tuning in all the phases of the creative work.

\section{Thematic Development of Slices of Life}

Thematically, the artwork Slices of Life took several turns. Originally I had intended to base it on the topic of (undoing) gender. However, I soon had to give this up, because the topic drew hardly any community to the blog. With the original arts team we then followed the ancient concept of the four temperaments that categorise human personalities into 'humours,' according 
to which each of the four types of humours corresponds to a different personality type. We incorporated the sanguine temperament that is associated with air in our artwork What if we had wings? This happened at a point when the building of and interaction with our community was still at an early stage. The compositional and performance gestures of the piece were rather abstract and strictly non-narrative. However, the call for entries that went along with it asked our community members to submit their visions and dreams in the form of short poetic statements, and this offered an opportunity to anchor the theme in their personal experience in a concrete way.

For the accompanying video I developed a simple, straightforward symbolism: an airplane that takes off from the runway and cruises above a vanishing landscape. I took the flight footage while travelling using a smartphone. The aesthetics of the video had a spontaneous and unrefined feel, and referred directly to the title of the piece What if we had wings? I placed the text submissions of the participants in the video. Using the flight footage was a first step towards a visual aesthetics that took its inspiration from the practices of social media users. I worked with material that anybody could have taken almost without effort: video shots from a vacation, captured to share with friends and followers on Facebook, YouTube or Twitter.

Link to the video documentation of What if we had wings?:

http://transcoding.info/english/book.html - PART I CHAPTER 2

\section{If I could only share}

what my mind's eyes see

through a camera lens

\section{Sharlea Taft, USA}

Fig. 2.5: Still from the video What if we had wings? which I premiered at the European Researchers Night in Vienna in 2014. The text is based on a submission by photographer and community member Sharlea Taft, USA. On the right: a runway stripe. Image (C) TransCoding

In contrast to the abstract musical score we had collaboratively developed as the then acting arts team, the words of the community and the symbolism I chose for the video were clear, straightforward and fairly concrete. 


\section{Singing shall be}

\section{My only and greatest love}

\section{And make my living.}

\section{Ernst Spitaler, Austria}

Fig. 2.6: Still from the video What if we had wings? which I premiered at the European Researchers Night in Vienna in 2014 . The text is based on a submission by community member Ernst Spitaler, Austria. In the background: the shadow of a starting airplane. Image (C) TransCoding

My colleagues were unhappy with the miscellaneous and diverse nature of the submissions, and with the concreteness of the visual and textual content. Concerns arose about the value of the community submissions. Moreover, the new power relations bothered them. It touched on their self-concept as artists working independently from society. My colleagues were worried that if they worked with the community material which - in their eyes - was not up to their usual quality standards, this might blur the image and careful branding as artists they had built over the years. As a consequence, they requested that we weaken the influence of the community by either letting them in only much later in the process, by exerting firm curatorial control over the submissions, or by processing contributions to make them 'fitting.' However, since this correlated with neither the original artistic and democratic idea nor the research mandate of TransCoding, we decided to terminate our work as an arts team and I continued as single artist in interaction with the community.

What made my situation different from the of my colleagues in the original arts team was the fact that to me, as a person originally educated as performer, the communication with the community was challenging but also worth the additional effort. It put me in touch with a new audience and helped me to think out of the box of the contemporary (art) music world and venture into new musical and aesthetic territory. Nevertheless, instead of being able to rely on pre-established quality assessments approved by my peer group, I needed 
to adapt and reframe my evaluation modes. I searched for a new aesthetic and conceptual approach in which I used the material authentically in a way that the often rough character of the contributions became a characteristic trait of and explicitly shaped the aesthetics of the developing artwork.

\section{Testing New Grounds}

Thus, the third phase of the project found me testing the grounds anew.

After first having asked the community directly about their interests:

"Who are you? Are you interested in writing, photographing, filming?

Are you a digital musician? a coder? a gatherer of thoughts?

What are your main topics when you blog/facebook/tweet/youtube/pinterest?

What or who are your passions and influences?

Which artist do you admire most?

And:

Do those topics have anything to do with who you feel you are?"

(Lüneburg, 5 Questions that are Important to Us 2014)

I now wanted to know what triggered their creativity:

"What gets your creativity going?

What motivates you to make art?

In which situation do you feel a creative flow?

Take a picture, write a sentence, write a short musical composition or just arrange some sounds." (Montrey, Call for Entries | What Gets Your Creativity Going? 2015)

And I listened back to the music of our followers when asking for remixes and compositions.

"what if? is calling for the craziest drones ever - and our audio archive provides just the inspiration. We've uploaded some inspiring audio files to SoundCloud and they're ready to be remixed into whatever your musical imagination will come up with." (Lüneburg, Call for Entries - Drone Remix Contest 2015)

I created artworks around these calls in which I experimented with aesthetics that were inspired by the contributions of the community. I looked into street art, and again worked with images that were produced with everyday means such as smartphone snapshots or webcam videos. As audio material, for instance, I used sounds from our SoundCloud that everybody else could compose with as well, or roamed the internet for interesting sound samples 
under a Creative Commons licence. I tested the resulting artworks Souvenir de Budapest, They speak in Layered..., The Gods Envy Us, Soundwaves and Mad Maddy on the blog, on Facebook, SoundCloud and YouTube for reactions from the community.

- Link to the videos Souvenir de Budapest, They speak in layered... and The Gods Envy Us: http://transcoding.info/english/book.html - PART II CHAPTER 6 (1)

A)) Link to the soundtracks Mad Maddy and Soundwaves: http://transcoding.info/english/book.html - PART II CHAPTER 6 (2)

However, these turned out to be blind alleys, not to be continued, because I couldn't engage the community with them. None of these works led to the one encompassing artwork that later would become Slices of Life, capturing the many facets of 'identity' we looked at on our social media channels. It all felt too detached from the project. In my opinion, the reason for that was that these artworks were about my struggle to come to terms with the challenges of the project rather than the community's visions of life and identity.

In contrast, Read me, the interactive audiovisual installation that I had also developed in this interim phase, was already very successful. The concept that individual community members were permitted to personalise the installation after their own taste and personality seemed to fit better with the community's wishes, the project's objectives and my own artistic expectations.

It was an artistic workshop with a group of students in Graz on 'undoing gender' that finally inspired me to bring back the project in a U-turn to the initial questions:

"What if we could live our lives regardless of the expectations of society, family, friends or colleagues?

What if our body, our gender wasn't our limit?

What if we took the freedom to be who we want to be and how can we express this in our art?" (Lüneburg, What-ifblog.net | About 2014)

I decided to build Slices of Life on narratives that depicted real (or fictional) life choices among our community members and their right and freedom to live and express them. I designed the popular and successful \#GYHAFYs challenge (\#GiveYourselfAHolidayAwayFromYourself) on which I based two slices of the show, namely Johnny and My Chosen Name is; I analysed the sound and text material of the personalised Read me installations for their expressive and story-telling potential and used them with permission from the respective community members, and did the same with the visual material we had received in the course of the project. 
Last but not least, I rounded the project off not only in the spirit of Judith Butler, who provided the initial spark for our project, but even more so in the spirit of one of our most active community members, the lawyer Gloria Guns from Canada. As text material for the final slice of life, I used what she calls the basis of her personal conviction and her professional work: Subsection 15(1) of the Canadian Charter of Rights and Freedoms. 


\section{MOtivation For PaRticipation}

TransCoding with its huge topic 'identity,' and its three performance domains the virtual online community interaction domain, my professional composing and live performing domain, and the research domain - makes for a complex social phenomenon and eludes simple categorisation. The key challenge was to gain an understanding of the motivational factors that moved the community from passive consuming to active participation.

We identified at least five models of engagement of our community:

- passive following of the blog and/or other social media channels;

- casual engagement in the form of liking or commenting;

- active involvement that had an impact on other community members' own creative work (for instance by using our SoundCloud to compose remixes, or by writing guest blogs on what-ifblog.net);

- interaction with me as professional artist and involvement in the shaping of the overall artworks Read me or Slices of Life through contributing texts, photos, voice recordings, sound samples or compositions; and

- sustained engagement with repeated participation in different challenges for different personal or project related purposes.

Attempts to explain motivational factors will necessarily face the problem of the disparate origin, age group and professional occupation of the participants. The general human subjectivity in recounting one's own actions and the tendency to individual self-promotion which showed in interviews we conducted with the participants (and which can often be observed in creative communities), add a further level of complexity to the analysis. So does the context of the digital, virtual setting that misses live person-to-person contact with the research subject. Moreover, different kind of calls for entries afforded different intensities of engagement, ranging from spontaneous, quick reactions to a long preoccupation with the creative process, which in itself made for different levels and kinds of motivation. However, in what follows I will present the findings that emerged from my participant observation, the analysis of responses to calls, and the small interviews we conducted with individual contributors.

\section{(Cultural-)Political Motivation and the Idea Behind the Call}

Different practices of engagement need to be considered in relation to the social and cultural context of the acting agents. Some participants simply supported the project out of (cultural-)political motivation. Among these were 
sound artists, young festival promoters (mostly from Eastern Europe) and politically engaged individuals. They contributed, because they backed the idea of open source and common creative practices (predominantly our users from SoundCloud), or they believed in outreach, diversity and the need to open up classical culture to as many people as possible. Some decided to explicitly make a political statement in answering the call to read and record Subsection 15(1) of the Canadian Charter of Rights and Freedoms, as we were working at a time of political upheaval about human rights in general and refugee movements in particular. Polish musicologist Monika Zyla for instance, a zealous protester for human rights and political freedom in Poland, translated the particular paragraph into Polish and sent us her personal recording. Anthony Green, a human rights activist and artist, mailed us a recording with an artistic twist which served as the rhythmic basis for the final section of Slices of Life, Charter of Rights and Freedoms.

A)) Link to the recordings of the Canadian Charter of Rights and Freedoms read by Monika Zyla, Anthony Green and Julien Charest:

http://transcoding.info/english/book.html - PART II CHAPTER 7 (1)

Then there were those who submitted contributions because they felt that the general ideas or the cause behind certain challenges resonated with them. Calls such as 'What Gets Your Creativity Going?' or 'Powerful Woman' seemed to trigger a motivational impetus rooted in experiences from the everyday life of our participants.

Sometimes people participated to explicitly support me in when I needed sound, photo or text material for Slices of Life, but sometimes they did so to just satisfy their own creative needs. As discussed in chapter 3, 'The Team Interviewed by Torsten Flüh', it mattered how challenges were presented and if they were able to prompt an emotional response in our community. When developing the artwork, and for this purpose designing the calls for entries, I tried to anticipate and respond to my users' preferences and areas of interest, since calls would only work if they were relevant to them and if I succeeded in matching their interest to mine, to mutually beneficial ends.

\section{Intrinsic and Extrinsic Motivation}

Apart from the social, personal and sometimes even political drive of our community members, we also found that intrinsic and extrinsic motivation were relevant to our task, and indeed were a key factor in triggering our participants' impulse to be creative and pick up the challenges we offered. We tried to implement this in our calls for entries, as I will describe below. 
According to psychologists Deci and Ryan, who developed the Selfdetermination theory (SDT), "[t]he most basic distinction is between intrinsic motivation, which refers to doing something because it is inherently interesting or enjoyable, and extrinsic motivation, which refers to doing something because it leads to a separable outcome." (Deci and Ryan 2000: 55) They define intrinsic motivation "as the doing of an activity for its inherent satisfactions rather than for some separable consequence. When intrinsically motivated a person is moved to act for the fun or challenge entailed rather than because of external prods, pressures, or rewards." (ibid: 56 ). Deci and Ryan argue that intrinsic motivation includes free choice and satisfaction, as well as interest and enjoyment of the activity; however, they also connect intrinsic motivation to the psychological needs for 'competence, autonomy and relatedness'. This leads them to propose that

"the primary reason people are likely to be willing to do the [extrinsically motivated] behaviors is that they are valued by significant others to whom they feel (or would like to feel) connected, whether that be a family, a peer group, or a society. This suggests that the groundwork for facilitating internalization is providing a sense of belongingness and connectedness to the persons, group, or culture disseminating a goal, or what in SDT we call a sense of relatedness." (Deci and Ryan 2000: 64)

In our project and especially in the calls for entries, we needed to set relevant and various stimuli to reach our heterogeneous community. In our Call for Entries - Drone Remix Contest, for instance, effectuated through our SoundCloud channel, we strove to balance intrinsic and extrinsic motivational factors. The call ran for three weeks from August 17, 2015 to September 7, 2015. We promoted the challenge on our main hub the blog what-ifblog.net, on our Facebook page (as a Facebook event), on Twitter, on Google+ and on our SoundCloud group, a feature that has now been abolished by the company. The original text for the challenge was as follows:

"What do you get when you combine a dysfunctional accordion, the beauty of Japanese wind bells and a sonified bubble speech?

what if? is calling for the craziest drones ever - and our audio archive provides just the inspiration. We've uploaded some inspiring audio files to SoundCloud and they're ready to be remixed into whatever your musical imagination will come up with.

To enter, create a wholly new audio experience by combining sounds from the what if? SoundCloud. Submissions may be any length, but must incorporate at least two of our tracks. (Deadline: September 7).

Maybe you'll grab a sound of nature (crickets from Croatia as your personal rhythm group), a selection from our synthesiser sounds, or a dark tune from our playlist 'Computer Blues'. 
Blend them together with your own music and then upload your drone remix to our SoundCloud group." (Lüneburg and Montrey, SoundCloud Group | What-ifblog.net 2015)

The text was formulated to stimulate intrinsic motivation by stirring the fantasy of possible contestants, emphasising the fun factor and calling for autonomous creative engagement with the free sound material we provided. The extrinsic motivational factors were the right balance between specified challenge and freedom to explore - not too many but clear instructions for the creative task - and the general framework of a contest and the community feeling on our social media platform. Participating in the challenge promised a chance to gain competence in audio creation, and on the other hand the sense of being related to and belonging in a peer group. Participants showed great investment and composed remixes up to eighteen minutes long.

As an example, contributor T.C. Elliott - who was one of our winners wanted to explore new compositional techniques, and the challenge fitted into his habitual creative behaviour, a sign for intrinsic motivation. Moreover, the contest came at the right time with the right kind of challenge, so he was also extrinsically motivated:

"I am participating in the 50 songs in 90 days songwriting challenge for the... oh, eighth year? So I am already in the 'let's write some music' frame of mind. I am wanting to get into midi recording and this drone challenge was a perfect opportunity. I don't generally use prerecorded track or do remix challenges, but this was specific enough (use THESE tracks) and still open enough (make a piece of music) that it seemed doable. I've only used drones in writing music one or two times before so this was still a new experience." (Elliott 2015)

Gloria had time to spare, the challenge triggered an interest that had already existed before (intrinsic motivation), and she found the extrinsic motivations of a deadline and the opportunity to share her work via our social media channels encouraging.

"Earlier this year on International Drone Day, I had the idea of making a drone piece about the Korean belief in fan death, but was too busy at the time with my job to do it. When I saw the remix challenge, I had just finished my work contract and had some time off before school was going to start, so I had some free time. I figured the remix challenge was a good opportunity to do the drone piece I had thought about earlier. I find that external motivators such as deadlines and an opportunity to share my composition (through your soundcloud/facebook/blog) really work well for me in terms of encouraging me to do something creative." (Guns, \#WhatIfDrone-Remix 2015) 
As a side note: Gloria, a Canadian citizen, has Korean origins and her remix Fan Death is rooted in her specific identity as she explains in a programme note.

"There is a persistent belief among Koreans that it is dangerous to leave a fan on while you sleep, because it might suffocate you. Sometimes I wake up in the middle of the night and can't fall back asleep, and all I can hear is the evil hum of the fan.

Composed as part of the Drone Remix project run by What If? (www.facebook.com/ events/ $1621294274814993 /)$, using several of their crickets and Gran Cassa samples, along with all of the fans in my house. Also featuring an old Korean lullaby [sung by Gloria herself], which translates roughly to:

'hush, hush, dear baby

you sleep so soundly...”"

(Guns, Fan Death 2015)

Gloria's participation was especially successful since not only did she produce an artwork that she found satisfying, but her soundtrack also found its way into two separate artworks of TransCoding: the audiovisual installation Read me personalised for Gloria herself and the segment Slices of Life - Korean Lullaby in the show for violin, soundtracks and video.

Link to the videos Read me personalised for Gloria Guns and Slices of Life - Korean Lullaby

(1)) Link to the remix Fan Death by Gloria Guns:

http://transcoding.info/english/book.html - PART II CHAPTER 7 (2)

\section{Relatedness and Belonging}

Another motivation for participation was the personal link to us as persons and artists. To consciously further this connection was part of my intention, when I worked on personalising our network-based communication. I supported direct exchange with our community members, and tried to remove the barriers of the (traditionally) hierarchical artist-audience relationship that is common in classical music. This led to several community members taking the opportunity to chat either with my colleague Montrey or with myself on Facebook, via Twitter or mail. Over the personal contact they shared their artworks, or they used the exclusive personal interaction to discuss how they liked our social media channels. Some even let us in on their personal situation in their home country. 
I, on the other hand, offered inside in my creative thinking, and in the problems and difficulties that occurred in the process of the project. In the blog post series Making of: Vienna Weekend Thoughts (1-3), for instance, I introduced the community to the ideas that inspired me for this project describing my fascination with the democratic idea of participatory culture while featuring Charles Leadbeater's inspirational YouTube video We think on mass creativity.

"Dear All,

What are my plans for this mini-series Vienna Weekend Thoughts? Today, tomorrow and on Sunday, I would like to introduce you to ideas that inspired me for this project, reflect a bit on surprises on the way and give you a prospect on what is planned next.

First of all, I would like to present to you Charles Leadbeater's YouTube video 'We think' [http://tinyurl.com/pcsjvp6]: 'The Audience is taking to the stage', 'People can have their say' and 'New ideas usually come through conversation' - These sentences carry a huge fascination for me and I was wondering if that could be transferred to our field of work, new music and media art. ...

And here we are now. In February our project officially started. Eight months later, our community has grown, gets involved, and talks to us. A first art work with texts of the community has been realised and our latest shelfie challenge is well on its way. We have had very individual, funny and arty submissions that beautifully reflect the personality of the people who submitted. Have a look at our what if? community gallery [http://what-ifblog. tumblr.com/] on Tumblr.

Today, I would like to ask you to shower us with more of your shelfies. We would like to weave them into a music video as the next part of our artwork on identity. Also, let us know what you think, keep talking to us, inspire us as we hope to inspire you.

Thank you for being part of this.

Barbara"

(Lüneburg, Making of: Vienna Weekend Thoughts 1 - We Think Therefore We Are 2014)

In the second post, I reflected on beautiful surprises during the creative and communicative process and hinted on difficulties on the way, and in the third post I gave them an artistic perspective on how community's contributions might possibly be integrated into the future artwork, while at the same time introducing them to art I loved. I did this all in the hope to connect on a personal level and to diminish the traditional distance between audience, that is our community, and myself, the professional artist and researcher. 
According to Deci and Ryan connectedness is also a feeling that is one of the possible positive outcomes when acting upon extrinsic motivation: Extrinsic motivation leads people to a sense of belongingness and relatedness that can be gained through their actions. \#WhatIfDrone-remix winner, Ken Ficara, explained to us his reasons for participating in the contest. Not only expected he to feed his love for drones (an intrinsic motivation), but he also mentioned that he felt connected with our aesthetic approach.

"I love drones, have hours and hours of them

I mean, drone pieces of my own. So that attracts me

However, the fact that I knew your work and I felt like I connected with your

approach/aesthetic

made me say yes

I knew the spirit would be positive, first of all

And your description sounded open/exploratory/fun, not dronier-than-thou"

(Ficara 2015)

'Relatedness' is one of the ground pillars that shape participatory culture on social media. In We-think: Mass innovation not mass production (Leadbeater 2008) Charles Leadbeater claims that people like to share on the web, because they socialise in online communities, they build together and get recognition for the work they do; they are not what they 'own' but what they 'share'.

Especially on Facebook, our community liked to enter conversations and share their experiences. Our question 'What gets your creativity going?' for instance triggered a lively and lengthy discussion about how to activate one's creativity amongst our community members and with us (Montrey, Call for Entries | What Gets Your Creativity Going? 2015). Moreover, it supported the feeling of belonging to a close-knit community and that of a shared identity: We were all creators. 'What gets your creativity going?' was a challenge that aimed for joint exploring and learning and which inspired participants and myself to do extra artworks on the side. Submissions took on the form of a creativity prompter on Facebook by Monique Besten from Belgium, (and my spontaneous artistic response to it), a guest blogpost on what-ifblog.net by Gloria Guns with a series of Arctic photos, and several compositions of members of our former SoundCloud group.

Concluding I state that TransCoding's online-communication and artworks was contextualised within a history of shared experiences which gave more meaning to our interaction. TransCoding offered a common goal and a purpose for coming together on our virtual platforms. It created social connections across different cultural backgrounds that would have not been possible to establish in daily life starting with geographical reasons, since the community came from four different continents. 


\section{The Act of Creation and Hedonic Rewards}

Peter Troxler emphasises the importance of 'hedonic' versus pecuniary rewards, namely learning, the act of creation, peer recognition and ego gratification, as motivational factors.

"The business, or rather, the benefits of commons-based peer-production are not uniquely monetary. The rewards include indirect mechanisms, such as the positive effects of learning, on future earnings or enhanced reputation ... The business also includes what economists call hedonic rewards: not consumption, but the act of creation gives pleasure to the prosumers. Peer recognition is another physiological reward, involving ego gratification." (Troxler, Libraries of the Peer Production Era 2011)

Government civil engineer Andy Getch, USA, participated in our remix contest and later in our \#GYHAFY call. When we interviewed him on his reasons for creating music, he not only listed hedonic rewards, such as pleasure in musicmaking, emotional gratification, admiration for musicians and the inner urge to grow into that role himself, but he also describes music as a core aspect of his identity.

"I make music because I love music, it moves me, a song is playing in my head most of the time. Music provides an emotional outlet, a means of expression when words alone don't. Music is a shining light in my life. I make music because I always admired the musician, the songwriter, and the lone performer in front of an audience. The lone singer-songwriter with a guitar embodies all three, and that is what I am now. I wanted to learn how to play the songs I love, then write my own songs. I make music because I am music." (Getch 2015)

Peer recognition and ego gratification was also an important aspect of participating. Some of the calls for entries were shaped as a competition and the winners were featured in special blog posts on what-ifblog.net that portrayed them as artists and persons. After having been one of the selected winners \#WhatIfDrone winner Sarah Sherlock wrote on her personal blog:

"I was one of the 3 winners! They had some great entries by producers and composers from Europe and the USA, so I'm really excited about it. This means my song 'Peripheral Vision' will be featured in an Audio-Visual Installation at the University of Music and Performing Arts Graz, Austria on 1 oth of October, an international meeting of artists and researchers and I will be also featured on a future dedicated blog post!" (Sherlock 2015)

On August 21, 2017, Susanne Wosnitzka commented on the Facebook page of the blog Dangerous Minds on the photo they used for their article on vintage photos of lesbian and gay couples. She proudly announced: 
"I used this main pic from this article [Fig. 2.7, below] to create a short story about 'Johnny' to give this supersweet couple a new (fictitious) history. Dr. Barbara Lueneburg composed the music for it - now it's a successful project who already run in Graz/Austria and Canada on music festivals. See and hear here the whole podcast of 'Johnny' in the 'Slices of life' project: https://what-ifblog.net/2016/11/09/johnny-and-other-stories/comment-page1/\#comment-4568"

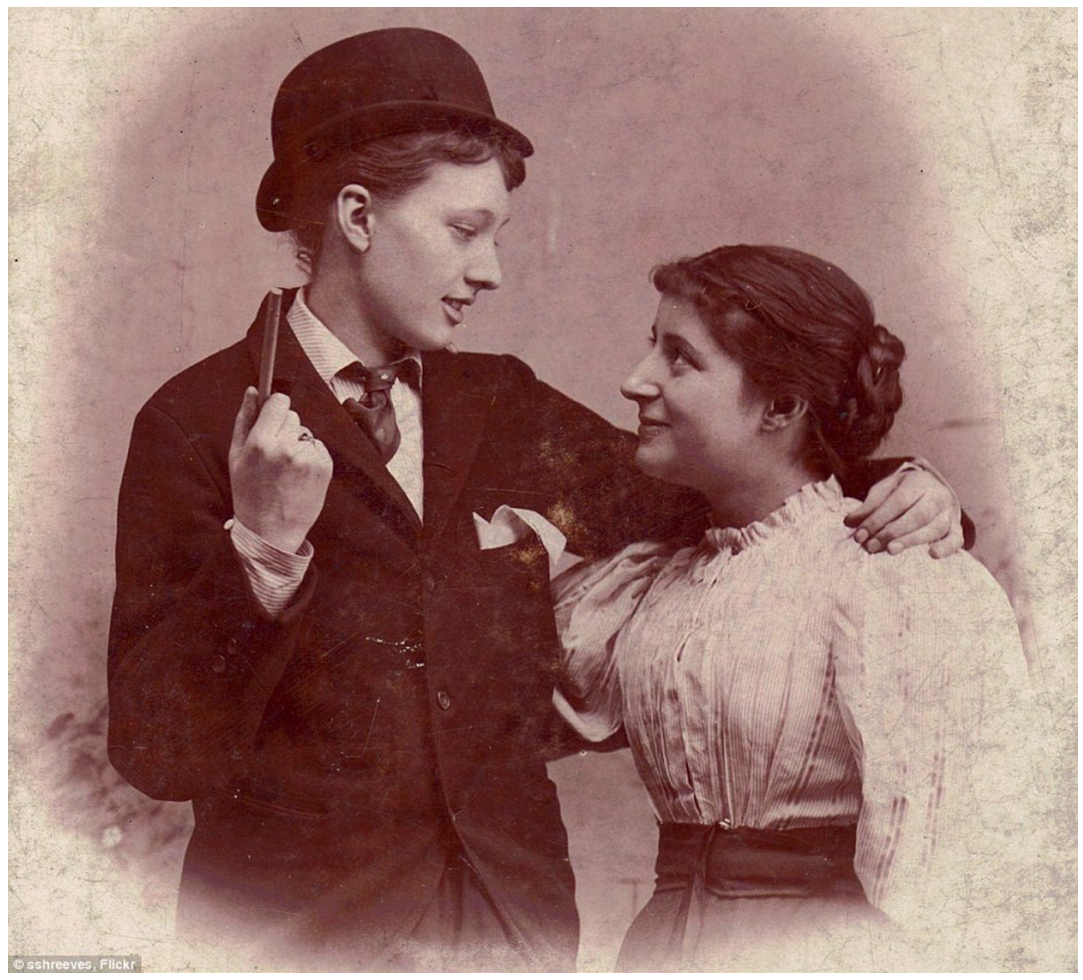

Fig. 2.7: Vintage photo of a lesbian couple that served as inspiration for Susanne Wosnitzka's story Johnny. The narrative became incorporated in the artwork Slices of Life. Photo source: https://www.flickr.com/photos/sshreeves/3424679723/, with permission of sshreeves

My team and I supported peer recognition in multiple ways. Community members were offered the possibility to express their personal views on identity in guest blogs. We featured the results of our calls for entries on the main blog what-ifblog.net. Every entry in response to a call was exhibited on the community gallery on Tumblr and, depending on the nature of the contribution, on Instagram (photo), SoundCloud (sound or composition) or YouTube (film scoring and variations of the personalised audiovisual 
installation Read me). Whenever I used material from the community in any of the artworks for TransCoding, or when community members personalised the audiovisual installation Read me, I first consulted the contributors to get their permission, and afterwards clearly acknowledged and mentioned them in any post on Facebook, Twitter, YouTube, both blogs, or in press releases and programme notes for live concerts.

Above all, I shared my pride when our participative art got national and international recognition by my peer group or by the community itself. From their reactions on Facebook and Twitter, it was clear that those members whose contributions became part of the final artwork got special ego gratification from that fact. On April 26, 2017, for instance, when I announced on Facebook, that the show Slices of Life was invited to Canada, Anahit Mughnetsyan (Armenia), Gloria Guns (Canada) and Susanne Wosnitzka (Germany), whose contributions to the show were substantial, commented as follows

“Susanne Wosnitzka: Wow - my story to Canada *impressed*

Anahit Mughnetsyan: I am so glad for all of us!!!! Our 'art identity' found it's way........ proud and honored to be part of it.....congratulations to all!!!

Gloria Guns: Hooray, you're coming to Canada! Welcome!”

(Wosnitzka, Mughnetsyan and Guns 2017)

In conclusion I should also point out that participants interacting within the same system, structure and content of TransCoding may have experienced a different understanding of what the project was to them, depending on their personal context and on their original intrinsic and extrinsic motivation for interaction. There was a clear range, from participation for the purpose of creatively expressing one's own identity (and through that connect with others), to consciously contributing to the show itself, to participating for (cultural-) political reasons, or simply for hedonic rewards such as the sheer enjoyment of being creative and the fulfilment when accomplishing a creative task. Ideally participants could achieve personal empowerment through their participation, would influence the artwork Slices of Life or Read me and support me with text, image or sound material to creatively work with.

My role was to offer content that met the requirements of our research project and at the same time encouraged the community to participate. I had to showcase classical contemporary multimedia art that was concerned with the topic of 'identity' in order to arouse the interest of the community as much as possible. The challenges I designed had to be of relevance for potential participants, anticipate their fields of interest and trigger an emotional response, while providing the potential that resulting contributions could be included into the project's artworks Slices of Life or Read me. The team's social media manager and I had to find and constantly improve communication 
structures on our social media channels to reach a wider, potentially interested audience and to keep up the flow of communication, information and creative incentives. We analysed the community's reactions to our calls, worked on anticipating the potential intrinsic and extrinsic motivational factors of our active members, and also featured the creative work of our contributors. As the person who ultimately incorporated the participants' contributions into the artwork, I had to make the influence and agency of the community clearly perceptible by picking up on their topics and respectfully integrating photos or images, sounds or texts. In this project I understood my role as artist as different from the traditional model in which the artist is exclusively concerned with developing his or her own inner ideas in self-directed effort, or trying to think and inquire independently from society or the audience, or creating art according to artistic principles that only well-informed insiders would be able to understand. In contrast, the creative and communicative work of TransCoding, both for the team and for me, was based on the democratic spirit of open source, of sharing among equals, on the free exchange of knowledge and goods, and on a joint creative endeavour.

Gloria Guns' entry in the remix challenge and its use in the artwork Slices of Life is an example of how community members had their own voice, expressed their specific identity, and felt satisfaction in the act of creation (and the freedom to do it in their own way), while I could match their self-interest with what the project and I needed myself. However, the process of integration was not always as easy as that. Especially in the beginning of the project, the initial artist collective struggled with the notion of including the creative say of the community in the overall artwork. I will expand on this topic in detail in chapter 9 ('A Potential for Change'). However, before I do so, I would like to feature the community's voice by presenting selected contributions by individual community members. 


\section{The Community's Voice}

The community exerted influence on the topic and shape of the artwork through the choice and composition of their narratives, imagery and sounds, and their interest in specific topics, which they communicated through their engagement or lack of it. Their influence shows in the fact that since the first sketches in 2014, when Slices of Life was planned as a contemporary (art) musiccentred work constructed on abstract symbolism around the Greek-Roman idea of the four temperaments (sanguine, choleric, melancholic, phlegmatic), it has turned into a narrative crossover performance based on a string of real and invented stories, sounds and photos contributed by individual members of the community. With their submissions our active members created various representations of identity - be it musical, photographic or textual. Some of these were so elaborate that I asked permission from the contributors to present them in this book. In addition to featuring them here I will seek for their meaning with regard to the artwork Slices of Life.

\section{Exploring the Notion of Spirituality}

'Spirituality,' as a part of our identity, was repeatedly made a strong theme on our social media channels. It eventually found its way into the artwork Slices of Life not only in the form of the segment I am a Priest (more about it in chapter 1 1, 'Case Study I am a Priest'), but also when I was given permission by Anahit Mughnetsyan to weave fragments of her spiritual song Megha (sung by soprano Heghine Ohanyan) into the final segment of Slices of Life. Armenian composer and choir conductor Anahit Mughnetsyan appears to be a deeply spiritual person. Her guest blog on her homeland describes how the ancient culture of her country, how land and landscape influence her identity, and how the place she feels is her place to be, and her creativity, both have roots in her upbringing and growth in what she calls "Mother-land+home+holy."

“Armenia-Homeland by Anahit Mughnetsyan:

I am a creator and that is my identity!

The place where I live, Armenia, is 'holy' or 'Noah's,' 'stone crosses,' 'paradise' ... land - they say.

Holy? Land? ...

What if the place we live in is really holy, unique, special...?

Why I love living in Armenia. Really don't know. No one knows why he's born, rises, lives, loves in the place which we call homeland...

Homeland... 
Home land or land home...The land is my home, yes maybe this is the key to the answers above. Each of us traveled a lot or just a little, it doesn't matter, anyway each of us can find the place where he or she can stay and feel like home+land... but when you travel Armenia you really understand the meaning of your identity, roots...

Identity? Roots? Again questions.

Yes and it's a pleasure to find your roots, and gain answers by walking on the holy+home+land... sometimes you find, sometimes you lose, but always you feel something special, unique for you in the air, from sunshine, from sand, from rain, from lightning, from everything. And what an interesting fact: I felt the same in Russia, Georgia, Iran...

So, then, what's my real home+land? Maybe it is the duration that makes a place my home+land? Because while you live in a place - for a short or for a longer time - in some places you grow, you learn, take and give at the same time; by the way, even from Motherland+home+holy... in that way: you with your circle of creations. usually you are just a jest in other places, except in your born+land=homeland, where your feelings grow from piano to forte, from soft to strong...

I love my Home+Land=World

What if the World is my home+land?

I appreciate Armenia where I take much and maybe give less, create a music, which is the voice of the land and that voice is unique, special, kind.... and maybe this is our identity to listen and reproduce it clearly and give back to the Mother-land for upcoming generations? Any identity is different and depends on the place you are at that time, and how deep your roots are...every time when you go for discovery and revelation, you take your roots with you, cause you're not a tree;

by the way ... as with each country, Armenia isn't an exception with its problems. As a newly reformed country (with over 2700 years of history and cultural heritage), its government does not pay much attention for today's art, culture, especially for music, but we've to remember that each of us has his or her own place and circle within. As I said above, I create music and I am not a composer, I am a creator and that is my identity!

Anyway, you know that: there is always a way to return home+land and the old door which waits for you to knock, which keys are always with you...

And finally I want to thank to my friend Barbara for being the reason again to think about my identity as a human being...

Here you will find some of my music from a live performance: Curch Ohanavan, VCC, 'Voxormya' (Pomiluy, Miserere) music by Anahit Mughnetsyan, камерный хор, худ. рук 
и дирижер Р.Мхитарян And click here for a video on my homeland Armenia: Welcome to Artsakh, ancient Armenian Land (original title: Karabakh a Hidden Treasure (https://www. YouTube.com/watch?v=3rs8Z_h6EYs)" (Mughnetsyan, "Armenia-Homeland" by Anahit Mughnetsyan 2014)

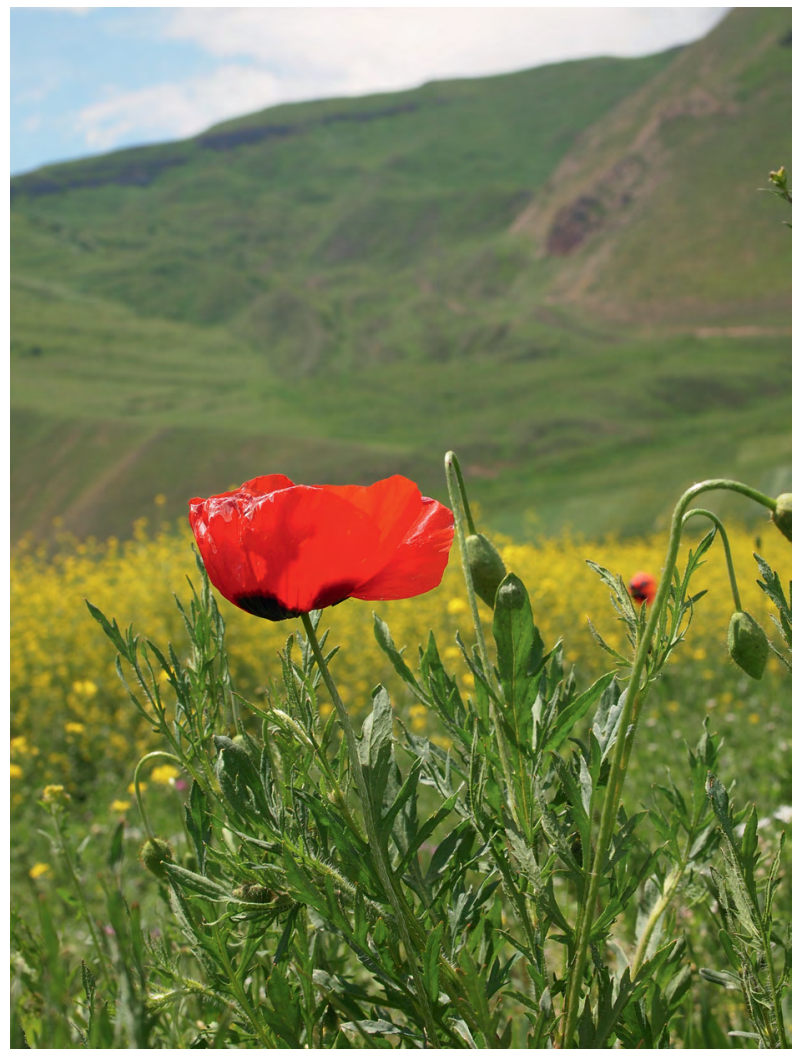

Fig. 2.8: Nature of Armenia close to Spitak. Used by kind permission of Zaruhi Babayan.

\section{Exploring Identity Through Words - \#GYHAFY}

My original wish to include Judith Butler's idea of "performing gender" as "a domain of agency or freedom" (Butler, Judith Butler: Your Behavior Creates Your Gender 2011) into the scope of TransCoding led to the call that received the most elaborate contributions from our community: \#GYHAFY - Give Yourself A Holiday Away From Yourself. The idea of the call was to freely pick a gender and script a self-chosen identity around it. The original wording of the call was inspired by weekend magazine questionnaires intended to entertain the reader: 
“Call for Entries: \#GYHAFY - Give yourself a holiday away from yourself

Take on another gender, another age, another name.

Your chosen gender:

Century of birth:

Country of birth:

Best friend(s)/(unusual) pet:

What did your parents want you to become?

What are you really doing now?

What is your day job?

What is your night job?

Where do you live?

- In a mountain hut/26th floor of a skyscraper/I own my house/I rent a room/I share a flat with (?)/...

- In the countryside/on a lone island/small city/big city/...

Where would you rather live?

If you were a plant lover/gardener/botanist - what would your

garden/park/meadow/field/balcony/window shelf/... look like?

You hate plants? Tell me about it.

What is the weirdest story of your life?

No story?

- Let me know what annoys you.

- Tell me what you love about life/this special day/last week...

Best encounter ever:

Favourite text/statement/book/...

Your chosen name is:

On what if?, we are looking for identities. Get creative.

Comment below on your imagined identity.

Join our FB event \#GYHAFY.

Or sent us an email with your chosen identity to barbara.lueneburg@kug.ac.at by the end of the month.

Yours truly,

Barbara"

(Lüneburg, Call for Entries: \#GYHAFY - Give yourself a holiday away from yourself 2016)

We could sense how the personalities of the individual writers enfolded and were reflected in their invented identities. Artists in real life stayed creative in fiction, 
singers became troubadours and fighters for human rights wrote stories about lesbians or transgenders. Linguist Lakoff and philosopher Johnson stress in their work that "metaphor provides a way of partially communicating unshared experiences, and it is the natural structure of our experience that makes this possible" (Lakoff and Johnson 2003: 226), and media and communication scholar David Gauntlett argues in Creative Explorations - New approaches to identity and audiences (2007)

"through language and other forms of self-expression (formations of the body, such as gestures or facial expression, or products of the body, such as drawings [or any other form of creating; author's note] we can make communications about our consciousness and our sense of being in the world which others can connect with and feel that we are living within a set of collective understandings and share some sense of collective experience." (Gauntlett, Creative Explorations - New approaches to identity and audiences 2007, 15)

In what follows, I will feature two \#GYHAFYs: Tala by the Filipina Feliz Anne Reyes Macahis and the scifi Piargno by the American Anthony Green.

Feliz Anne Reyes Macahis is a composer and singer from Manila, Philippines. Tala stood out from all the other stories that were submitted, because Feliz not only invented the character of Tala, she also sent us three of her own drawings as part of the story and composed music for it.

“\#GYHAFY | Tala

Your chosen gender: Female

Century of birth: 21st century

Country of birth: Canada. Well, my mom is Asian and my dad is European, and they were travelling a lot. So I was born in Canada, but I grew up in Scandinavia.

Best friend: his name is Emma. He's my kindred spirit. I've met him in one of my trips years back and if I can remember correctly, we've only seen each other few times - all fleeting moments! But we constantly talk and write letters. Snail mail is the best!

What did your parents want you to become: To be a musician.

What are you really doing now: making a lot of money.

What is your day job: my job is to tell you what you want to hear. You can call it Advisor (but I prefer naming it as ' $\mathrm{B} * 1 \mathrm{lsh} *$ tter'). It's getting better and better, though. My clients used to be just from U.S. and Europe, but now I'm expanding in Asia. 


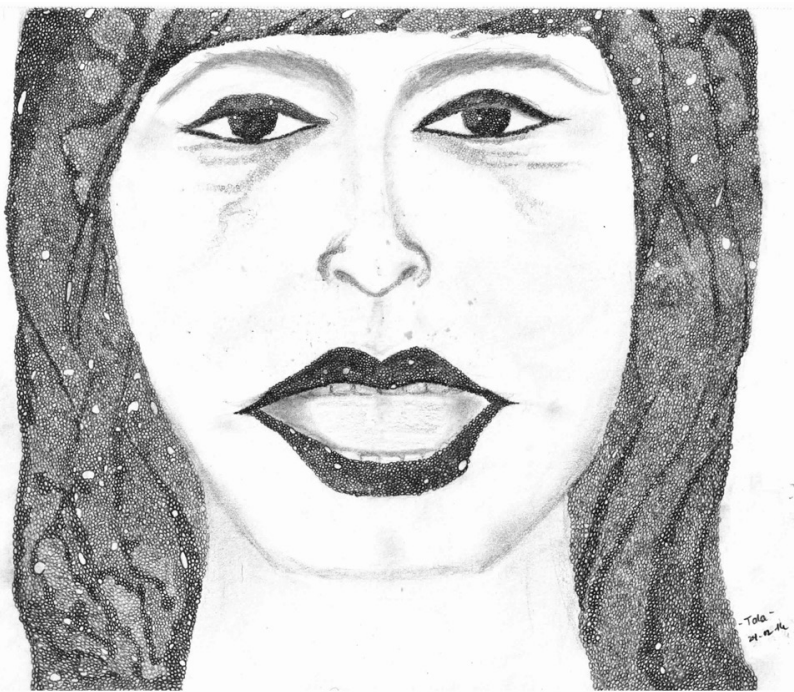

Fig. 2.9: Portrait of Tala (C) Feliz Anne Reyes Macahis, by kind permission of the artist.

What is your night job: to assist my dad in his social experiments or to give my mom some ideas for her next composition - basically: to be a daughter. I have a great relationship with my parents. I can be myself; talk to them about anything; and even complain why people are stupid. Haha, you have no idea how this topic could go way deeper once I start the discussion with my dad!

I spend the rest of the night sketching some ideas or images.

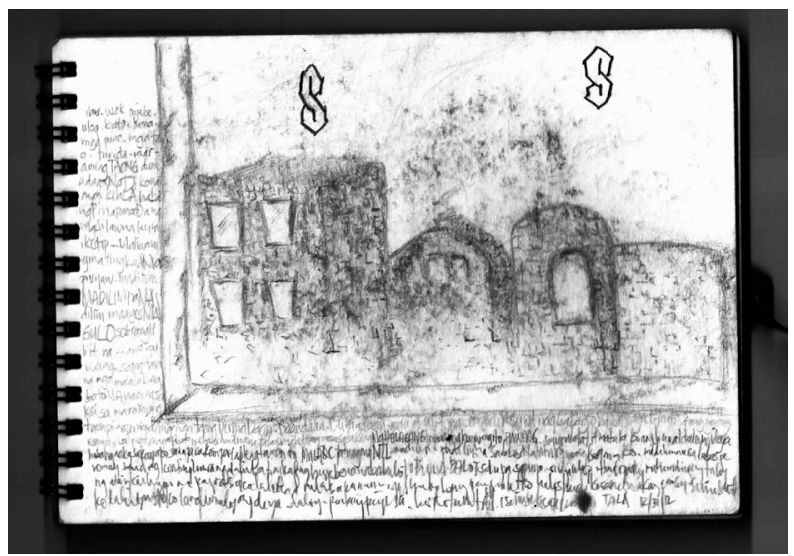

Fig. 2.10: Illustration for the blogpost Tala, (C) Feliz Anne Reyes Macahis, by kind permission of the artist. 
Where do you live: I have a big flat at the city center.

Where would you rather live: closer to my parents' house - near a pond where I could build my own boat house.

If you were a plant lover/ gardener/ botanist - what would your garden/ park/ meadow/ field/balcony/window shelf... look like: I love flowers! My window shelf is full of orchids.

What's the weirdest story of your life: I have a twin! I only learned about it when I turned 20. Seriously, that long! My dad wanted to conduct his experiment and have me and Bituin as his guinea pigs. He published his book about the study last year.

Another thing is that I make weird drawings. I have so much love in my heart, but my drawings say something else. Can anyone read me, please?

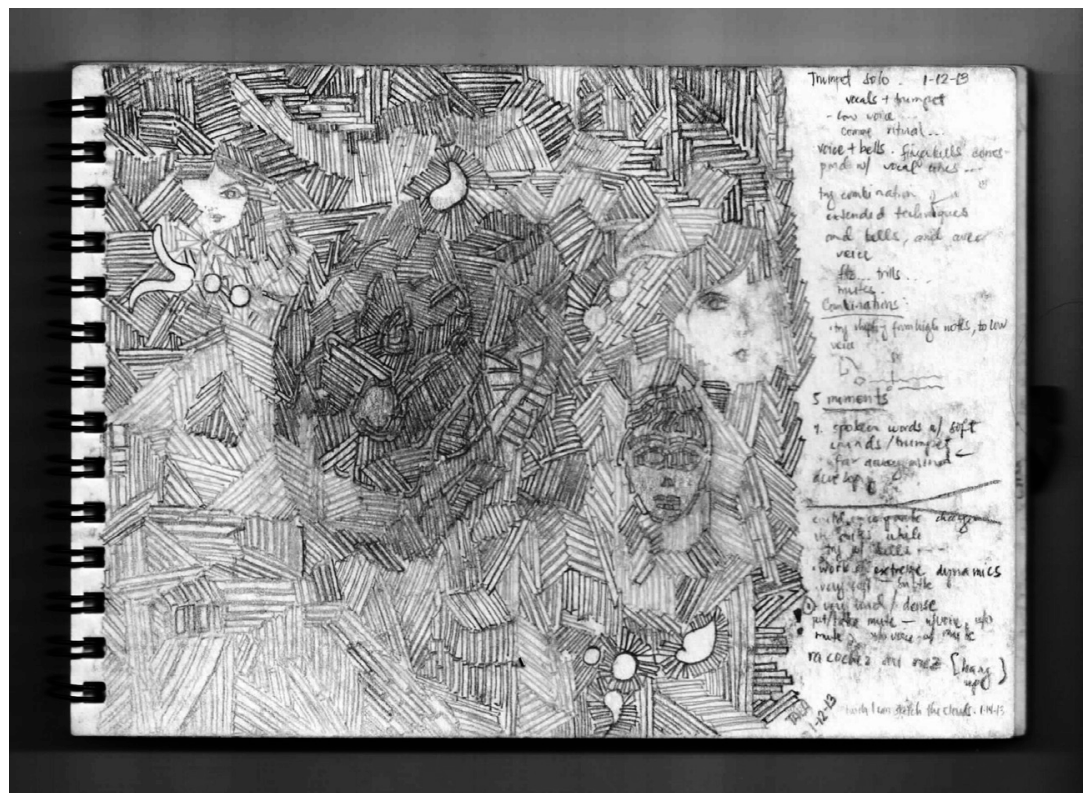

Fig. 2.11: Illustration for the blogpost Tala (C) Feliz Anne Reyes Macahis, by kind permission of the artist.

What annoys you: My clients. A lot of them are dumb. If they use their common sense, they don't have to pay a lot of money for my advice.

Best encounter ever: When I was 12, my mom brought me to her concert. They performed a piece of her that is 10 years older than me! It was like being in a different world. 
A)) Link to the madness of the night and the woven stars by Feliz Anne Reyes Macahis: http://transcoding.info/english/book.html - PART II CHAPTER 7 (3)

Favourite text/ statement: 'Everyone gets so much information all day long that they lose their common sense.' - Gertrude Stein

Chosen name: Tala"

(Macahis, \# GYHAFY Tala 2016)

Another contributor, Anthony R. Green, describes himself as composer, performer and fighter for social justice. On his personal website he states that "[b]ehind all of his artistic endeavors are the ideals of equality and freedom which manifest themselves in diverse ways in a composition, a performance, a collaboration, or social justice work.” (A. R. Green, Anthony R.Green 2016) Anthony picked up on my incentive of gender and invented a male transgender, whose favourite book is the Declaration of Independence.

“GYHAFY | piargno

Your chosen gender: Transgender male

Century of birth: 8 oth

Country of birth: Intorion (transliterated)

Best friend(s)/(unusual) pet: a tardigrade

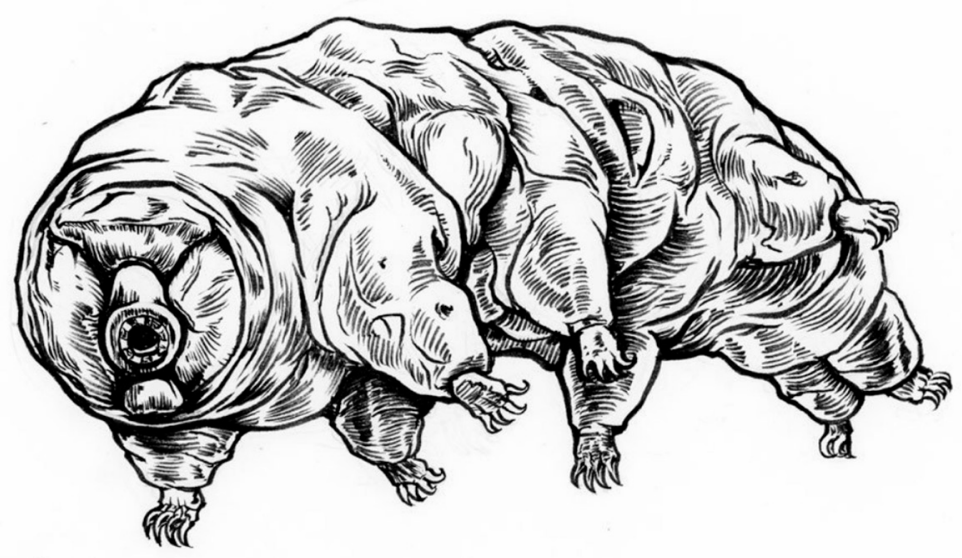

Fig. 2.12: Tartigrade by Phineas Jones on flickr under a Creative Commons license. 
What did your parents want you to become?

They had no interest in my life. I was raised by computers, but not the ones with the ability to care, just the ones who provided the basics.

What are you really doing now?

Creating a vehicle to explore the latest discovered star in Croteo Lurnexa

What is your day job?

Engineer and interpreter

What is your night job?

Participant in a life-long sleep study

Where do you live?

In a pod in the center of the big city Frotorx, in a floating building that has 500 other individuals living in pods. The digital simulations make it more spacious than it sounds. Where would you rather live?

In an underwater hotel on planet Earth.

If you were a plant lover/gardener/botanist - what would your garden/park/meadow/field/ balcony/window shelf/... look like?

Full of black flowers.

You hate plants? Tell me about it.

I don't hate plants. I just don't belong to the cast of people that gives them any thought or attention.

What is the weirdest story of your life?

When I was in an online relationship with 7 other people for 6 years, I could not escape the desire to get plastic surgery to physically change my appearance, rather than rely on the digital version of myself that I had created for my partners. It was the first time I ever considered the old theory that digital representations of your physical self - that don't actually match your physical self - are lies. I ended the online relationship, and began a non-digital one with someone who lives in the pod complex. We are still together - we've been together for 9 years, but we only see each other and talk to each other about once or twice a month. It's the best relationship I've ever had.

\section{Best encounter ever?}

One day when I was researching tips on entertaining my tardigrade Loozy, I was contacted by a composer named Frips Chathrameh. I guess he found out that I am a big fan of his scent music. He told me that he also has a tardigrade, and it is also entertained by his scent music. I never thought of that, but it worked like a charm.

Favourite text/statement/book/... The Declaration of Independence 
Your chosen name is: Poh Mia"

(Green, \#GYHAFY | piargno 2016)

\#GYHAFYs found their way into Slices of Life in two different forms. Firstly, through the segment Johnny, the love story of a lesbian woman. The author, Susanne Wosnitzka, lent her own voice as my fictive interview partner, while I scored the text. Secondly, in the segment MY Chosen Name, which featured six Mini-GYHAFYs that people gifted to me on Facebook.

"My chosen name is Gloria.

I was created in 1994.

I like slapstick comedy and I'm not ashamed to admit it."

(Mini-GYHAFY by Michael Wolters, Great Britain, contributed on the author's personal Facebook site on February 22, 2016)

These Mini-GYHAFYs were recorded by members of the community to become part of the composition.

Link to the video documentation of Slices of Life - Johnny:

http://transcoding.info/english/book.html - PART II CHAPTER 7 (3)

A)) Link to the audio recording of My Chosen Name is

http://transcoding.info/english/book.html - PART II CHAPTER 7 (4)

\section{Exploring Identity Through Sound - Calls for Audio Entries}

During the runtime of TransCoding we initiated three calls for sound in total. Just before New Year 2016, we asked for submissions of downloadable sound samples as a 'holiday' gift to our community to celebrate the impending New Year. In response to this call alone, we received forty-one submissions from our twenty-seven group members. As we had just sixty-four followers on our SoundCloud at that point, it means that $42 \%$ of our followers followed our call. They were a small group, but they were deeply engaged.

“Call for Entries: Holiday Gifts | \#WhatIfSoundSamples

Dear All,

Head on over to our SoundCloud, because we've left something under the holiday tree for you. Students from Barbara's 'Undoing Gender' course at the University of Music and Performing Arts Graz have been hard at work coming up with ideas that represent gender dynamics and images. The result of one of these thought exercises is 'Screams and Growls,' 
in male and female editions: (1)) https://soundcloud.com/what-ifblog/sets/screams-andgrowls

Now we'd like you to ask you for a small holiday gift for our community, as well. Submit a sound sample under a Creative Commons license so that any members of the community maybe use and remix them. Submit directly on our SoundCloud group:

https://soundcloud.com/groups/what-ifblog-submissions, Deadline: December 29.

We'll put all your submissions in a playlist, and then we'll pick our favourite three sounds to create a special 'late-under-the-tree' blog post. But most importantly, we can all remix each other's contributions for holiday/New Year's remixes and get some What if? community spirit going. We would love that ...

Thank you very much and happy festivities,

Your what if? team."

(Montrey, Call for Entries: Holiday Gifts |\#WhatIfSoundSamples 2015)

German DJ and installation artist Denim Szram contributed a free sound sample that reflected in its musical shape and development almost exactly what he described as creative process typical for him:

"My compositional process normally starts with my experimenting with synthesizer and sound recordings, searching for interesting textures and moods. Once I find something interesting I try to expand this and build a variety of sound in different frequency ranges. From low sub frequencies up to high noisy sound, from long pads to short signals. With these different elements I then try to build a dramaturgy, to keep the listener excited. (Szram, \#WhatIfSoundSamples | Denim Szram 2016)

New York based Laura Feathers expresses her creative identity through various outlets such as photography and text, and contributed to almost all calls for entries on what-ifblog.net. An electrician by day, Laura extends this to her free time with an electronics hobby. "My knowledge of electronics from my work overlaps with the technical side of music and with the rich history of electronic music," she wrote to us. "I am passionate about exploring new approaches to sound." (L. Feathers, \#WhatIfSoundSamples | Laura Feathers 2016) Her offering for \#SoundSamples was a track under the artist name Magic Sun. Reminiscent of vintage synth work of decades past, the sound world that Laura created developed into much more than a simple sound sample, a piece almost four minutes long titled Soundscape 1: 
(1)) Link to What if? Free Sound Sample by Denim Szram:

http://transcoding.info/english/book.html - PART II CHAPTER 7 (4)

(1)) Link to Soundscape 1 by Laura Feathers aka Magic Sun:

http://transcoding.info/english/book.html - PART II CHAPTER 7 (4)

Laura's approach tied in with the call \#WhatIfDrone-Remix Contest in which we invited our participants to use sound samples from our SoundCloud to creatively express their musical identity and style.
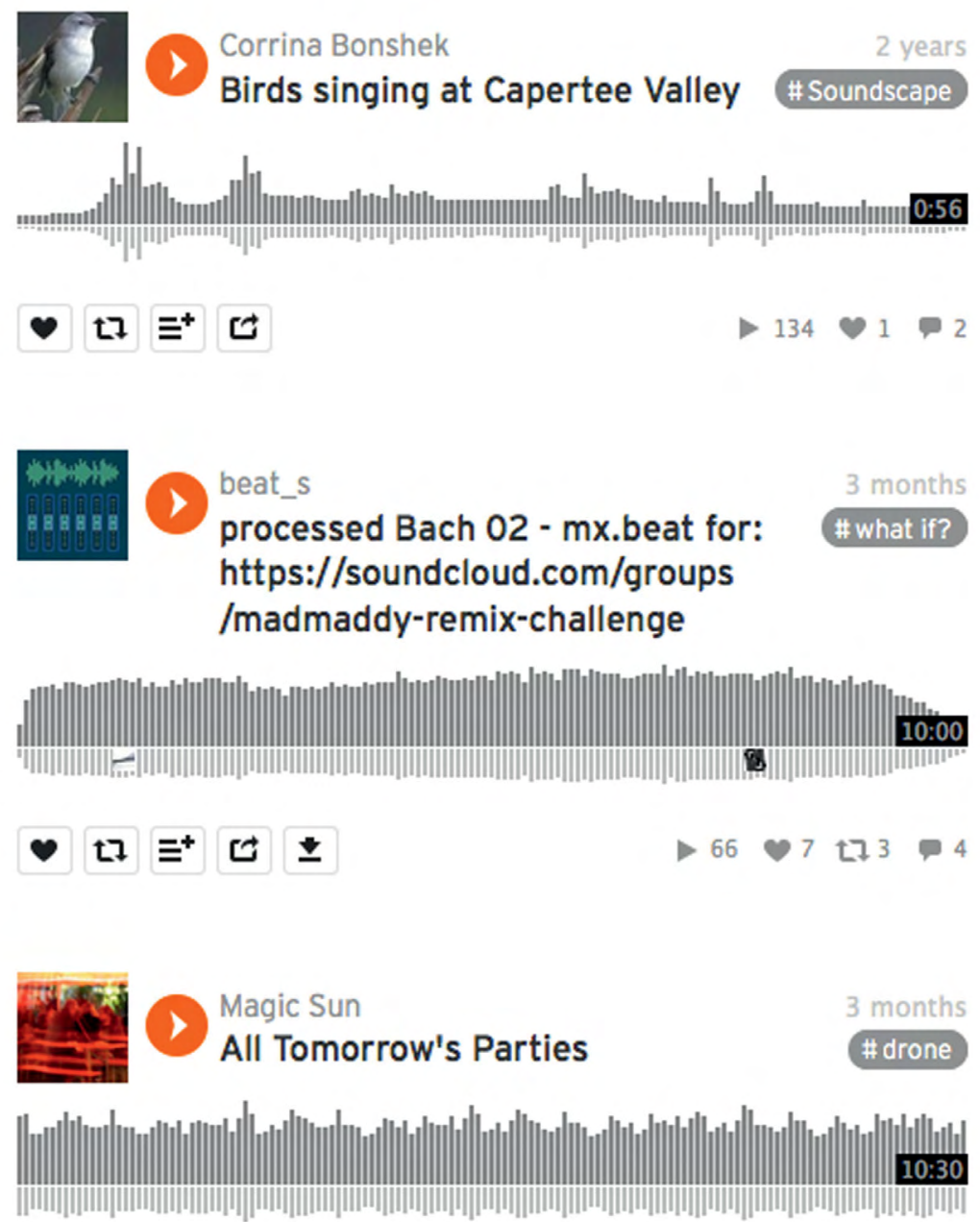

Fig. 2.13: A screenshot from the SoundCloud group of TransCoding showing Call for Entries: Drone remix with contributions by Corrina Bonshek, Beat S and Magic Sun. 
With the third call for audio entries I explicitly addressed our community to help me with the artwork for Slices of Life. I asked our members to record themselves reading Subsection 15(1) of the Canadian Charter of Rights and Freedoms:

"Every individual is equal before and under the law and has the right to the equal protection and equal benefit of the law without discrimination and, in particular, without discrimination based on race, national or ethnic origin, colour, religion, sex, age or mental or physical disability." (Canadian Charter Of Rights And Freedoms Subsection 15 (1) 1982)

We received contributions in English, French and Polish. The original motivation for using this specific text came from Canadian community member Gloria Guns, mentioned above. She wished to include a subsection of the Canadian Charter of Rights and Freedoms as part of her personalised Read me installation. As a human rights lawyer, Gloria considered this subsection of the charter a substantial part of her own personal identity. For my part, I thought that a statement about equality and the right to freedom was a fitting idea to round off the series of identities that formed Slices of Life and accordingly I incorporated the recorded voices into its last segment, Charter of Rights and Freedoms.

\section{Exploring Identity Through Imagery - Frames of Life}

Our calls for images were almost as popular as the calls for texts. Uploading snapshots on Tumblr, Facebook, Twitter, Pinterest or Instagram has grown into an almost ordinary activity with avid social media users, so contributing appeared to be easy, spontaneous and playful for our participants as well. We asked for shelfies, Catrinas (see below) and pictures of powerful women, and we showcased photographers who captured moments of everyday life in their work.

One of the photographers featured was Romanian artist Sabina Ulubeanu who was a supporter of TransCoding and a regular participant in our challenges. In her visual art, she loves to frame scenes she 'finds' on the street. Not only are her photos carefully chosen in terms of colour and formal composition, but each motif tells a specific story. For instance, her image of an old woman behind a window. We just get a glimpse of her. The worn window frame adds its story to the woman's life.

Through her photography Sabina makes the particular quality of this moment stand out, so that we, the onlookers, can sense it as well. Her series 'frames of life,' became the visual basis for the video of Charter of Rights and Freedoms in Slices of Life. 


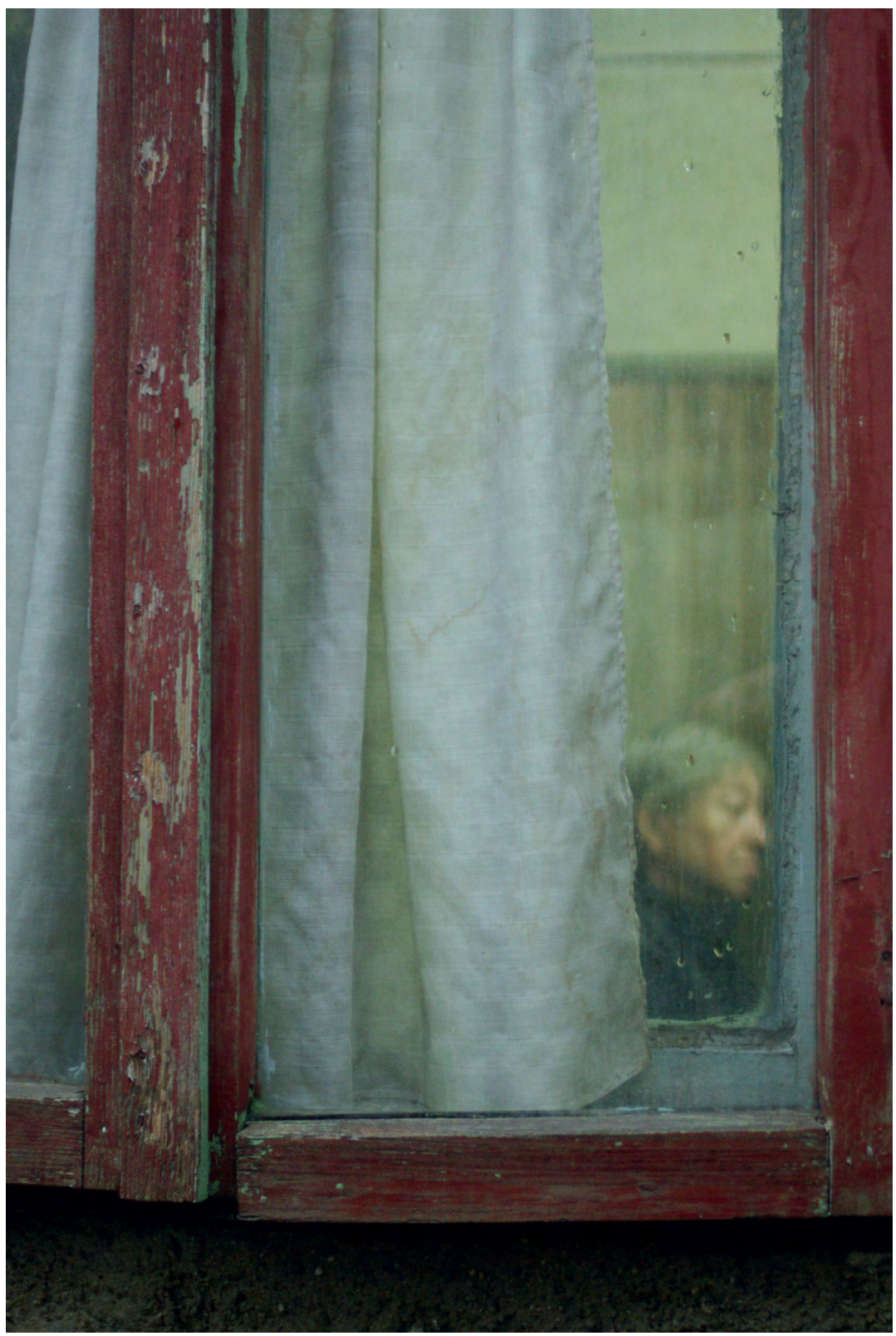

Fig. 2.14: Old woman behind window (C) Sabina Ulubeanu (by kind permission of the artist) 
In response to the above mentioned Call for Entries: Creativity is Contagious in which we asked the community for shelfies that expressed their creative identity, one participant, visual artist and professional engineer Hali Rey, sent us a poetic arrangement consisting of leather-bound books, a statue and text fragments.

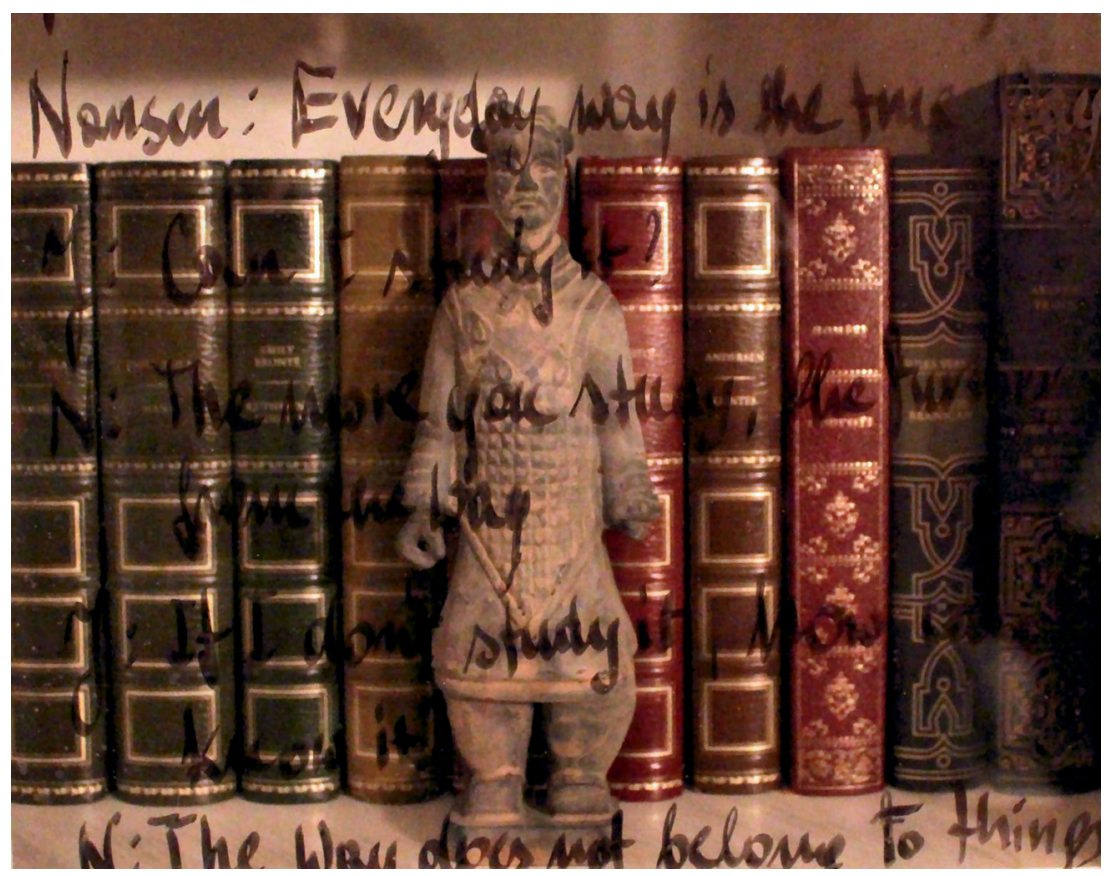

Fig. 2.15: Shelfie by Hali Rey, submission for the Call Creativity is Contagious (by kind permission of the artist)

The magic of the picture reminded me of my encounter with young Russian pianist Alina Murzakhanova, and her love for literature and classical culture. Alina was one of the community members who got a personalised version of the audiovisual installation Read me. When I met Alina, she talked about art and life and we exchanged experiences of what it is like to live in a foreign country. Living abroad sometimes reminded her of the world of the famous story of Alice in Wonderland: the grotesqueness of situations, the fractures in everyday life that come from being a foreigner, the uncertainty of right or wrong, those are questions she felt she was confronted with while living among people of another country. For her the text by Lewis Caroll encapsulated all this, and she chose it as the basis for her personalised version of the audiovisual installation Read me. 
In the soundtrack for her installation, the clear ringing of Asian bells express the dreaminess of Alina's world, claves capture her determination in life, and sounds of an old, broken accordion transport us right into the middle of her version of her own personal wonderland. I derived all the sound material for Alina's soundtrack from TransCoding's SoundCloud. Later, I used the text and the soundtrack of her installation as the compositional source for the segment Who am I? in Slices of Life, and Hali's shelfies became the backdrop for the corresponding video. Already in the first months of the project threads had appeared that connected individual contributions and the interests of the community members and myself.

Link to the videos Read me personalised for Alina Muzakhanova and Slices of Life - Who Am I?:

http://transcoding.info/english/book.html - PART II CHAPTER 7 (5)

The next call for entries, Catrinas, which we launched on occasion of the Mexican Day of the Dead, was tied in with the notion of spirituality, which as we have seen was one of the undercurrents of TransCoding. Additionally, it picked up on the topic of national festivities as an expression of a nation's identity. La Catrina, the famous female skeleton dressed in rich clothes, is a key element within Mexican culture.

"Call for Entries | Catrinas

Around the world, people are dressing up and preparing for celebrations related to All Saints' Day, such as Halloween. We thought we'd tell you about one folk identity and its related traditions, and from that, we could create a fun call for entries! ...

In Mexico, Día de los muertos celebrates our deceased relatives and friends, while keeping their spirits alive in our memories, and making them offerings of food and gifts. Based on an etching by Jose Guadalupe Posada, La Calavera de la Catrina has become an important figure expressing the spirit and folk identity of Mexicans on this holiday, and many people take inspiration from her for costumes and crafts ...

We'd like to see your take on Catrina! This can take any form you like: a drawing, a painting, a dressed-up figurine or doll... in fact, some of us have bona fide Catrina dolls lurking on our windowsills; Barbara's resident Catrina is pictured here! Alternatively, you can paint your face and take a selfie, or just wear clothing that suggests the spirit of the holiday." (Montrey, Call for Entries | Catrinas 2014)

The Mexican Día de los muertos, the Catholic All Souls' Day, the Japanese Bon Festival and the Korean Chuseok are festivals that honour the dead and 
celebrate death, possibly in an attempt to overcome fear of mortality. In a followup blog post Montrey reflected on the call and its connection to our topic of identity:

"What does Catrina have to do with identity? First, most generally, it is a form of artistic self-expression. Secondly, a Catrina is an expression of facing death, of accepting death as inevitable. Yet there is something satirical in this ... The figure of the Catrina, though portraying a dead person, is at the same time very much alive: she is animated, she wears colourful clothes, she dances. In the face of death, we are all equal, yet we all celebrate the preciousness of life through looking death in the eye."

(Montrey, Catrinas | Submissions and Thoughts 2014)

As a representative sample of the many Catrina submissions we received in response to our call, Fig. 2.16 shows Daniela Michelle's drawing which, together with a selection of shelfies and other Catrinas, found its way into one of the associated artworks for TransCoding, the music video The Gods Envy Us.

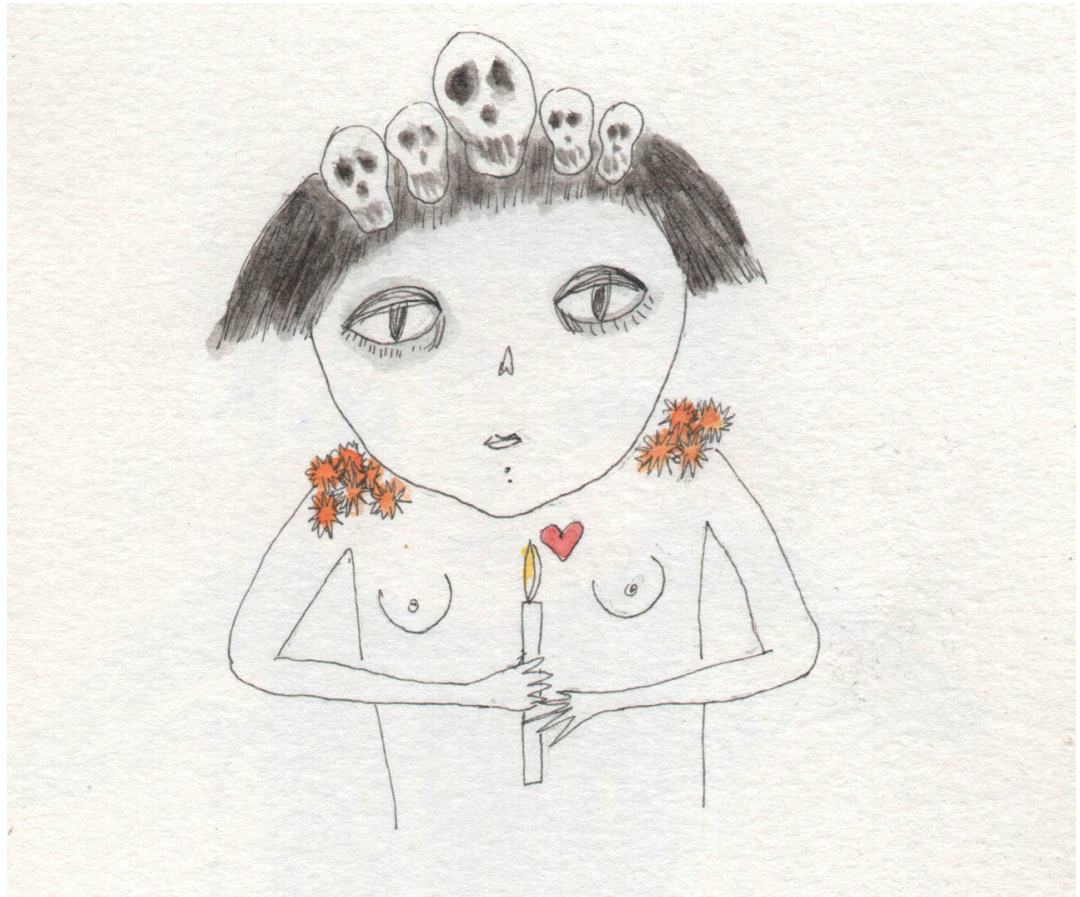

Fig. 2.16 Daniela Michelle's drawing of a Catrina, in response to our Call for Entries / Catrina 
The video is based on a text quoted by one of our Facebook followers on her personal Facebook page; it was allegedly said by the Greek warrior and hero Achilles, of Homer's Iliad: "I'll tell you a secret. Something they don't teach you in your temple. The Gods envy us. They envy us because we're mortal, because any moment might be our last. Everything is more beautiful because we're doomed. You will never be lovelier than you are now. We will never be here again."

- Link to the video The Gods Envy Us:

http://transcoding.info/english/book.html - PART II CHAPTER 6 (1)

The community contributed in word, image and sound, as well as inspiration. We aimed to offer calls that illuminated the topic of identity from different angles and offered interesting choices for our members to engage with. We started from personal visions in our call 'Contemporary Fairy' (discussed in detail in chapter 9, 'A Potential for Change'), included daily observations everybody could make such as in the photographic series 'frames of life,' touched on philosophical and spiritual questions, included national holidays, as a way to feature a nation's identity and asked our members to express their creative identity through music, word or image.

Participatory art necessarily poses questions around authority and decisionmaking power. Among others the agency and authority of our community consisted in the acceptance or denial of the incentives we offered, in the performance of their own creative practice when reacting to our project, and last but not least in their particular interpretation of our calls (possibly diverging from my original intentions) that subsequently guided my creative decisions. On the simplest level, challenges or topics that didn't elicit any answers I didn't further pursue in the artwork. Those challenges that received a lot of attention, on the other hand - such as \#GYHAFY - found their way into Slices of Life and gained meaning for the further development and aesthetics of the overall content.

However, the recursive process of input-output directed from us to the community and vice versa delivered not only an interesting creative interaction but also rather powerful affective experiences, especially among the original group of artists. In the following chapter, I therefore investigate in detail the following question: how, how much, and on what level did I afford our participatory community authority in the decision-making process of our joint creative work, and how did this affect the artist(s) involved and the community?

(Note: chapter 9 is adapted from an article submitted to the Finnish online journal on artistic research RUUKKU: Lüneburg, From ,Highbrow Art' to Participatory Culture - A Potential for Change 2017). 


\section{A potential for Change}

This chapter deals with the question of shared authority and the impact of the interaction with the community on the role and self-concept of the artist. I first analyse in detail the second main artwork of TransCoding, the audiovisual installation Read $m e$, with regard to concept, mode of participation and the question of authority shared with individual community members.

\section{Case Study Read me - an Area of Success}

Read me is an interactive audiovisual installation that can be personalised for individual TransCoding community members. The original artistic idea and the conceptual and technical framework came from me, but individual community members were invited to personalise the installation for themselves by filling the framework with their own chosen texts, portraits, sounds or even compositions. The afforded authority lay in the actual content: the material provided to turn the installation into a person's personalised artwork.

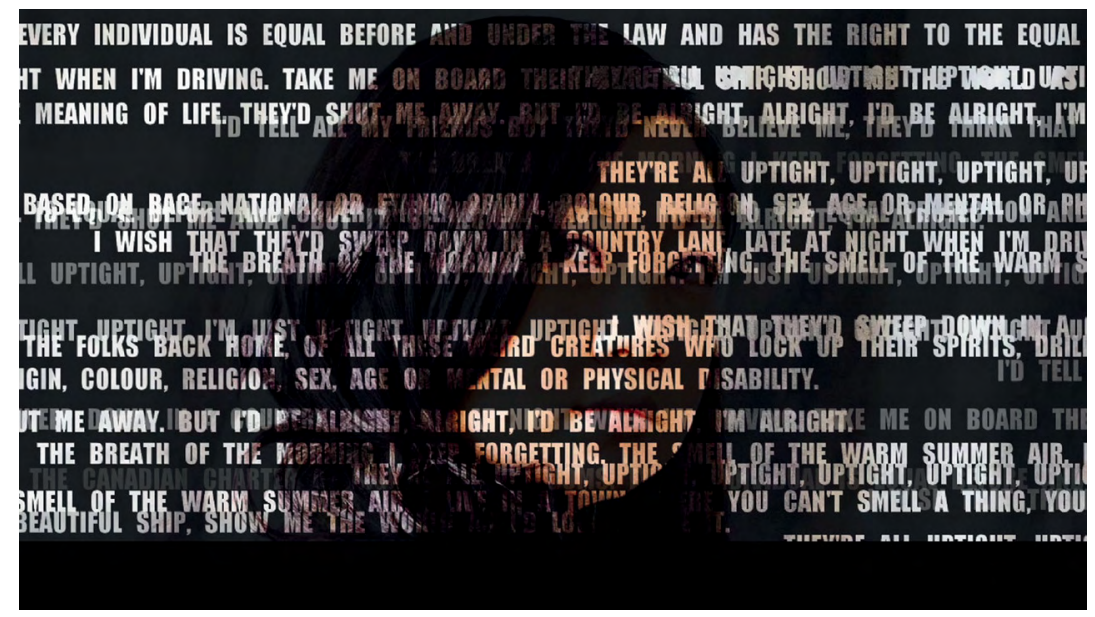

Fig. 2.17: Still of the audiovisual installation Read me in the personalised version for Gloria Guns (October 2015). Image (C) TransCoding

Concept and composition - Barbara Lüneburg. The musical material I composed with is in great part derived from Gloria's contribution to the Call for Drones placed by TransCoding.

Photograph of Gloria Guns: (C) Denise LeBleu

Choice of text material: Gloria Guns

Software programming and visual advice - LIA, Damian Stewart and Marko Ciciliani. 
The idea behind Read me is the following: Often our first impression of a person leads us to believe that we can grasp who this person is. It seems clear and obvious. Yet the closer we get acquainted, more and more complexities about this same person are revealed. In the installation Read me, the closeness of the relationship is expressed through the distance of an audience member to the projection. The further away one is, the clearer the material will be (at its limit, one soundtrack and one sentence visible on the projection). The closer we get, the more complex and layered the material will become. The audio and the visual content of Read me reflects the complexity of our impressions of a person. (A playlist of the Read me installations can be found at the link bit.ly/ $1 \mathrm{KqVTCd}$. The videos trace the approach of an onlooker to the installation).

To personalise Read me for a community member, I tried to get to know them, preferably personally, but if that was not possible, I made a connection online. In the standard version of the installation, I subsequently asked the community member to send a photograph of themselves along with texts that were close to their heart. For the installation, I overlaid the image with a dark layer, so that an onlooker can only see the shadow of an image behind the overlay. One single sound layer is audible and one single sentence appears in the middle of the projection. The letters of the sentence take away the dark layer and the underlying portrait shimmers through. This symbolises the first strong impression we have from a person when we have a sense that we already clearly know who this person is, when in reality we are usually still very much left in the dark (Fig. 2.18).

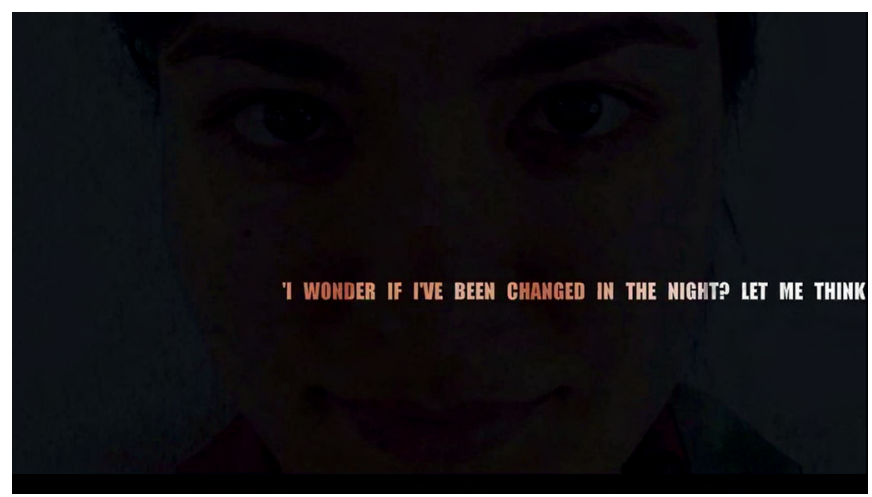

Fig. 2.18: Still of Read me (2015) in the version for Alina Murzakhanova. The onlooker is far away. Image (C) TransCoding

Having met the same person more often, we get to know more layers of them and start to see more clearly. Accordingly, when the onlooker approaches 
the installation, further sentences appear and along with them more of the photograph becomes visible. The audio gains a second layer as well. (Fig. 2.19).

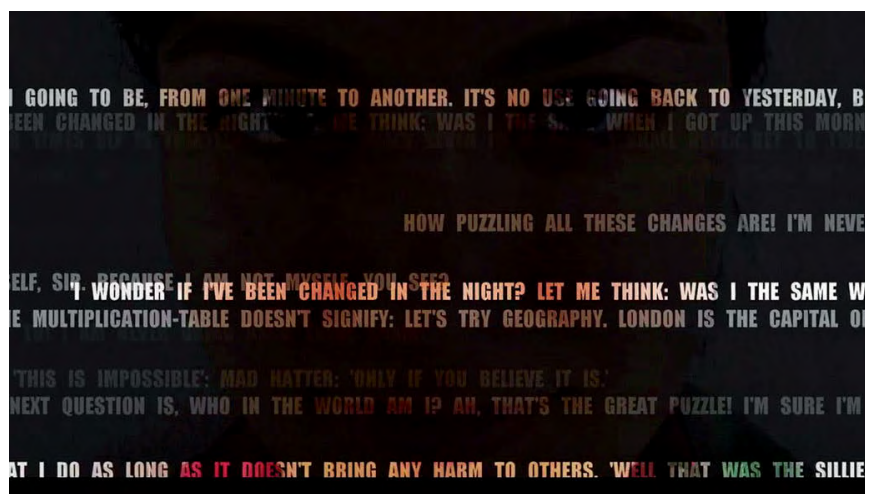

Fig. 2.19: Still of Read me (2015) in the version for Alina Murzakhanova. The onlooker comes closer, more layers of text, music and image appear. Image (C) TransCoding

Once the onlooker is very close, all the layers of music, and all the layers of text appear. The amount of text almost covers the screen and uncovers the image. Now we can see the person in the picture behind much more clearly, yet the texts are not necessarily legible anymore, the content is more shrouded. The audio on the other hand reveals more characteristics of the person represented. The onlooker, sees more, learns more about the person, yet has a sense that they don't know him or her as clearly as in the beginning (Fig. 2.20).

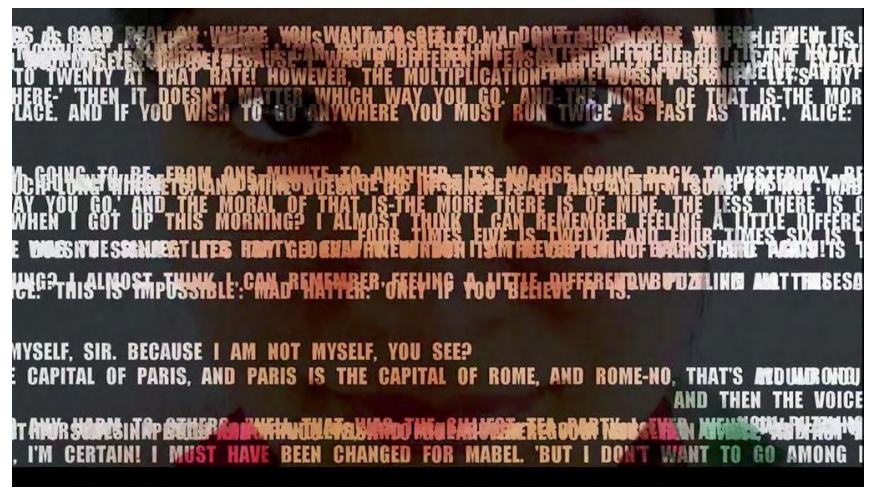

Fig. 2.20: Still of Read me (2015) in the version for Alina Murzakhanova. The onlooker is very close, all layers of music, image and text are perceived. Image (C) TransCoding 
In what follows, I retrace in detail the decision-making power, that is, the authority, that I granted users when I opened up the installation to them. I do this by looking at the categories of material they contributed and by assessing the degree of involvement of individual community members. Additionally, I briefly analyse the reasons why I consider the Read me iteration featuring Ricardo Tovar Mateus to be especially successful.

\section{Community Authority in the Creation of Read me}

The modes of working for the installation ranged from what Nina Simon calls "contributory: the participant supplies content, the artist incorporates it in the artwork," to "collaborative: the participant and the artist are committed to deep partnership," to "co-creative: the artist is committed to supporting the needs and goals of the participant that align to the project and provides them with the necessary tool to accomplish the work." (Simon 2010: 190 f) Five versions of Read me have been realised in total. Depending on their creative self-assessment, selfconsciousness and keenness to be involved, as well as their technological skills, the community members could supply one or all of the following categories of material for the installation: image, text, raw sound material and a composed soundtrack.

When I gave individual community members access enabling them to personalise and creatively work with the installation, I was aware that this could eventually lead to aesthetic frictions between them and myself, which I would possibly find difficult to overcome. Not everybody shares my taste in music or text, and vice versa. Community members might not like what I composed for them or might not find themselves represented in the sounds I chose. In the worst case we could mutually dislike what we had jointly created. In the case of community member Maria Hippenfels [name changed by the author], to whom I will introduce you later, some of the possible frictions showed.

To begin with, I would like to illustrate the degree of decision-making authority through a graph that conveys on which level (x-axis) and to which degree (y-axis) a community member has decision-making power when personalising the audiovisual installation Read me. The last column is derived of the arithmetic average (the sum of the different categories of material provided by each community member divided by the total number of categories).

Regarding the material that could be provided, everyone accorded high priority to images. In just two cases I had been offered a series of portrait photos to choose from (accordingly, I assessed this as granting a lesser degree of authority to the participants). Text seemed to be equally important to the community members. Only in the prototype for Montrey did I add a second text 
by the National Union of Women's Suffrage Societies to the one she had chosen. As I knew Clio personally it was my interpretation of Clio Montrey, and she feels that I adequately represented her in the context of the artwork. Maria's choice of text was also slightly supplemented by me, since we needed more text material for her installation.

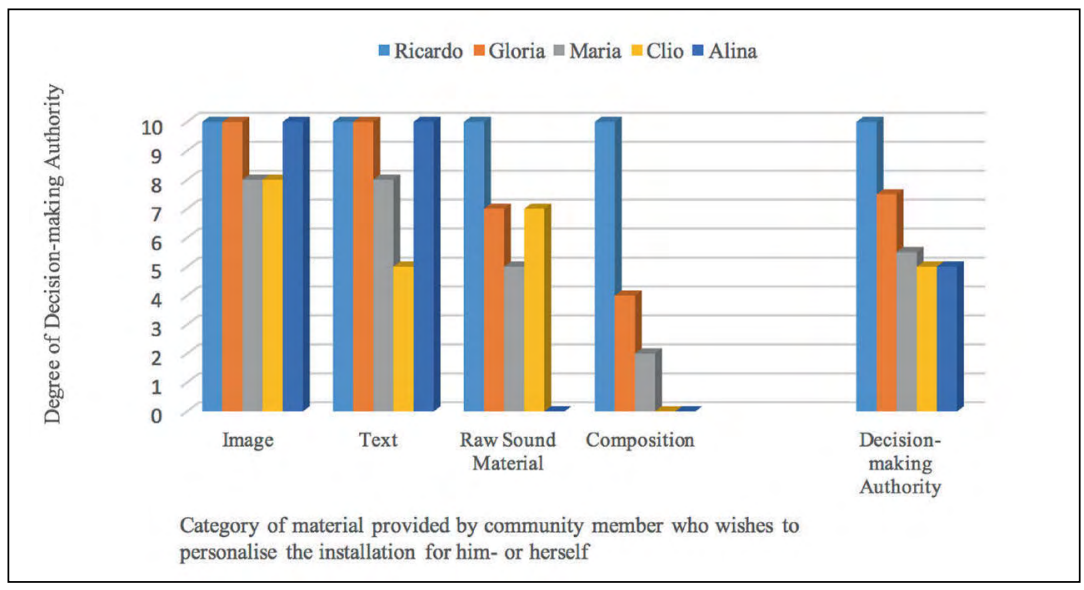

Fig. 2.21: Graphic to convey the degree of decision-making authority granted when Read $m e$ is personalised by individual community members. Image (C) TransCoding

From everybody except Alina Murzakhanova, a Russian classical pianist, I also received raw sound material to work with. In Alina's case, she had no piano available to record sounds in situ when we met, and she herself had not enough technological experience and equipment to later provide me with sound material in a digitised form. The sound material contributed by the portrayed community members was usually very personal and added to the individual and unique feel of each single installation.

The compositional process to put together the actual soundtrack yielded the widest range of technological and compositional experience from the community members, which is probably why there was less community decision-making involved. Again the prototype for Montrey is somewhat of an exception, since with her expertise she could have composed the soundtrack herself. However, at that point in the installation's development, the framework and overall aesthetics of the installation had not yet been fully implemented. It was only in subsequent versions that the idea emerged to offer the subjects the option to create the soundtrack themselves. In the case of Gloria Guns, the lawyer and pop musician from Canada, the raw material for the soundtrack of her installation consisted of a remix she had done based on material drawn from our SoundCloud tracks (described in detail in chapter 7, 'Motivation for Participation') and my personal music: 
A)) Link to the remix Fan Death by Gloria Guns that served as the raw material for her personalised version of Read me:

http://transcoding.info/english/book.html - PART II CHAPTER 7 (2)

For Maria we recorded her voice and djembe improvisations. When composing the soundtrack for each of their installations, I was dealing with pre-composed elements, which were inherent in the material they had provided. Therefore, I assigned a percentage of authority to them over the composition of the soundtrack, although I actually composed it myself.

There is only one person who provided every element for the installation, resulting in a comprehensive contribution: Ricardo Tovar Mateus, a young composer and pop musician from Colombia (Fig 2.22).

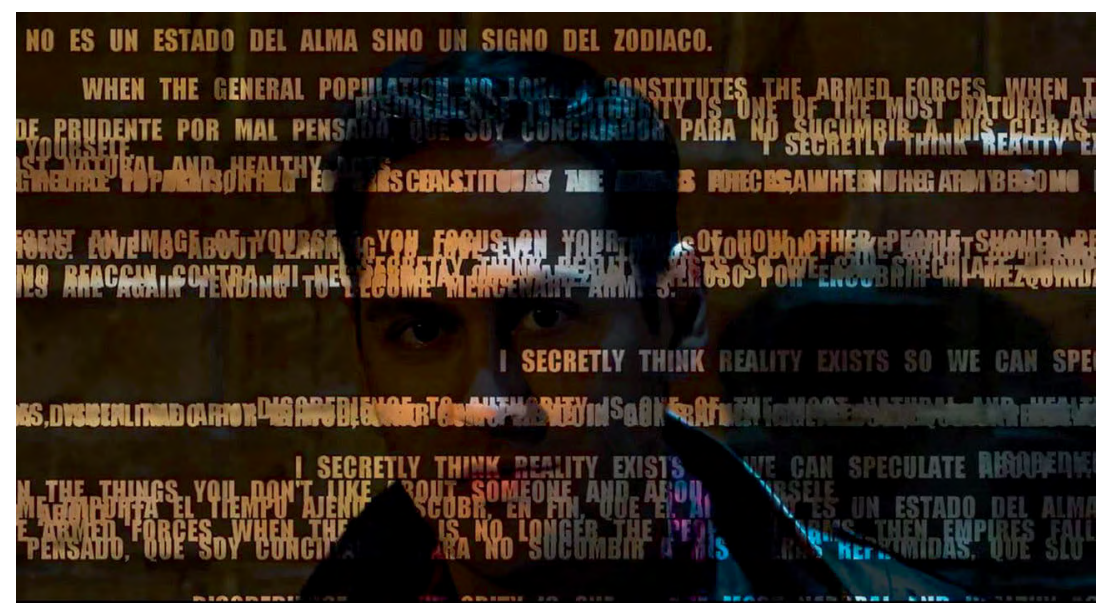

Fig. 2.22: Still of video: Read me in the personalised version for and by Ricardo Tovar Mateus (2015). Ricardo was the only participant who provided all the elements for the installation: image, choice of text and composition. Image (C) TransCoding

He successfully took $100 \%$ authority for the material he wanted represented. Why did it work so easily with Ricardo? First of all he fitted perfectly into our target group: at the time of our co-creation he was a bit over 30 years old, educated in 'high art' but working in popular culture. He is technologically savvy and is a skilled composer. He had moreover contributed to two previous calls for entries since autumn 2014, so he had a good understanding of what this project was about.

Link to the video Read me personalised for Ricardo Tovar Mateus: http://transcoding.info/english/book.html - PART II CHAPTER 7 (6) 
When calculating the arithmetic average of the percentages in all categories, we found that community members took on at least $50 \%$ of the minimum given authority. The individual contributions were clearly framed within the artwork, recognisable and acknowledged. Each installation had a unique feel to it while conveying an impression of the person we saw and of what was dear to their heart. It captured an idea of the multitude of layers that make up their personality.

What were the advantages for both the participant and artist in setting up the installation in a participative manner? First of all, there was the individual value of empowerment: The installation explicitly empowered community members to express their own identities and to actively participate in the creative process. The conventional power structure changed: the usually hierarchic relationship between artist and audience became one of permeability and mutual influence, and the participant had a self-chosen degree of agency in the creative process.

There was a learning value for both the community member and myself as artist: the audience member who co-created the installation was involved in the creative process, learned about new media art from the inside and fostered his or her sense of accomplishment; for me as the artist who set up the artistic framework of the installation, the learning value lay in the variety of personalities I portrayed, the diversity of material and topics that were offered to me, the possible frictions in taste and goal that I had to overcome, and in the resulting artistic challenges. Last but not least, there was the social value: the artwork was dedicated to the people who worked with me on the installation, the installation travelled with me across Europe, I documented it on our YouTube channel and shared peer recognition with them. From their reactions we gathered that it boosted their feeling of self-esteem to be portrayed by me and to be given agency in the process. As a result, the relationship between artist and audience was reinforced far beyond the encounter in a museum or a concert hall (as would more traditionally be the case in the new music scene).

In the next chapter I outline the possible areas of conflict that our project yielded, analysing in detail the development of the multimedia composition What if we had wings? from the perspective of the original arts team. To let the reader more clearly grasp the context and the complexity of the setting I will refer to the method of 'thick description' as developed by ethnologist Clifford Geertz in The Interpretation of Cultures (Geertz, The Interpretation of Cultures 1973). For this purpose, I will look in close detail into the actual process of developing the artwork and include discussions among the group of artists involved. 


\section{Online Community and Original Arts Team in Opposition: An Area of Conflict - Case Study: What if we had wings?}

As mentioned in chapter 6 ('The Art Production') the multimedia composition What if we had wings? for violin, text-based video, live electronics and soundtrack includes text contributions of our online community that were incorporated into the artwork of the team. The call that rendered the text material was called Call for Entries / Contemporary Fairy. We introduced it on our blog with a short fairy tale, followed up by instructions on what kind of contributions we were looking for. We chose the form of a fairy tale, because most people can relate to these as part of their cultural upbringing:

“Call for Entries | Contemporary Fairy

Once upon a time, there lived an artistically inclined polyglot fairy (though she herself disliked that loaded and complex string of labels, because she felt that labels limit how one can express one's identity). She went around the world looking for ways to facilitate artistic collaboration.

What if you were to meet this fairy on your way to the office or to your studio, and she told you she could grant you one wish and make one of your dreams come true? This dream could be anything: personal, professional, artistic, or a wish for society at large...

We would like to gather entries in the following form: One short text, no longer than one sentence. This text can be in any format you desire: a coherent, grammatical sentence, a haiku-length poetic-type written statement, a string of words that can be understood, a graphic poem made with simple word processing means such as use of spaces, special characters, capitalisation, etc. This sentence should tell the audience what your dream (vision) is. It can be personal, professional, creative, or a view for society at large. Your 'wish for the fairy,' if you will."

(Montrey, Call for Entries | Contemporary Fairy 2014)

We received multiple comments on the blog and contributions via mail or Facebook. 


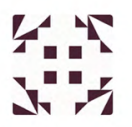

Sandra Allain August 13, 2014 at 3:32 pm

I wish time could be a pulse

a fragmented timescale

a soul traveling impulse

between imagination and reality

I wish time did not dim

memories and silhouettes

plastering wrinkles

where emotions are untold

I wish life

I wish to live

http://www.sandraallain.com

Fig. 2.23: Entry for Call for Entries / Contemporary Fairy by Sandra Allain on what-ifblog.net.

This was the first participation project we initiated that was extremely successful in terms of contribution volume and scope. However, the inclusion of the contributions in the artwork brought to the surface major conflicts among the original arts team on the notion of participation.

To trace the origins of the conflicts I will recall the sequence of events that led to What if we had wings? in detail and include excerpts of a discussion among the original group of artists. The arts team had developed the soundtrack for this piece in the context of collaborative sessions between February and August 2014. We created it entirely without relating to the community, but we knew the community had to be involved in our artwork at some point. Consequently, Call for Entries / Contemporary Fairy on the blog was meant as a resource of material for a video to go along with the music, whereby the material should be provided by the community. The call was initiated about a week after our last working session. I was to premiere the music (with the video) on European Researchers' Night in Vienna on September 26, 2014.

As it was not technically feasible to hold another arts team gathering between August 1 and the premiere, we discussed the call, the material it rendered and the making of the video online only. Soon some feelings of unease and discomfort came up. (In the following quotes I will refer to my fellow artists with imaginary names: Anna Veenen, Bernhard Hütten and Connor Willing). 
Anna Veenen: "The questions I have could be described in general as questions on the balance between 'our show' and the 'participating culture.' ... I don't feel very comfortable with this way of working, and I feel less 'participating' in the project, because it gets further and further away of how I normally work ... I definitely see the many values of the project, of the blog, the growing participation etc, but speaking for myself I am quite incapable to 'participate' in this way. I am not a blog writer, I have no experience with participatory art nor do I feel able to make an artwork which includes many materials by other people. The reason that I participate in this project is because I want to make a show with you three, and I underestimated the participatory part, which is not the way that I work." (Anna in an email to the author and the rest of the team, on June 16, 2014)

Due to time pressure and to the fact that the other team members were either gone for their summer vacations or busy in their usual professional work context, I took on the task of developing a video for the European Researchers' Night and incorporate the text material we had received. I used footage from a plane flight taken on my smartphone and depicted the community texts as they had come to us without shortening them, refining them or processing them further to make them seem more 'artful.' To do so was a conscious decision of mine, because I wanted our contributors to feel that we appreciated their work as it was and would use it as authentically as possible.

- Link to the video documentation of What if we had wings?:

http://transcoding.info/english/book.html - PART I CHAPTER 2

While the performance garnered praise from the community, it didn't meet with the approval of my colleagues. This consequently led to a fundamental discussion between the members of the arts team about the participatory set-up of the project in general and the inclusion of the community in our artwork. In our discussions we searched for what it was that my fellow artists felt worked against the notion of community participation and for the source of their resistance to the basic concept of TransCoding.

To understand what happened it is important to follow a timeline that starts even before this project. The setting for an artwork that predated TransCoding was different from the artistic research setting of the project itself. Originally the arts team with its four members from the fields of composition, performance, visual art and interactivity had only agreed on a joint collaboration on the topic of 'Undoing Gender,' which aimed to develop a performance for violin, electronics and visuals. Everybody had agreed to be part of the group and of the project because each person wanted to work within this special group of four artists hoping that our collaboration would result in an interesting and rewarding artistic endeavour. Nothing more, no research attached, no community involved. 
We explored possible avenues of funding. The research funds we received for TransCoding afforded us the necessary means to get the project off the ground, yet at the same time called for a completely new angle to our work: the idea of letting an online community participate.

Initially none of us had realised how deeply the participatory process with the community would eventually concern us on a personal and on an artistic level, and to what extent it would be necessary to engage with it. It had an enormous and unexpected impact on our approach to creating art. We discussed the consequences of it in an extensive team meeting.

Barbara Lüneburg [the author]: "We [Connor Willing, Bernhard Hütten and I] have worked together before. We [addressing Anna Veenen] have never worked together before, yet I have always admired your work. And I think what we did in the summer, at the end of July and the beginning of August, was something really, really special. Yet, I am torn. I am in the middle of this community thing and on the other hand I feel a responsibility towards you. I can feel that you have to move too far out of your comfort zone, which you didn't expect, which I didn't expect and I don't feel comfortable asking you to do that [interacting with the community] but on the other hand I don't know what I can do. [Nods from the others]" (Team discussion on 4 th of October 2014).

We were seven months into the project, and I as project leader had necessarily (and willingly) already become deeply immersed in the interaction with the community we were building.

Barbara Lüneburg: “... unexpectedly - this whole community thing grew much more quickly than I thought. It grew to my heart, it is something, as I said before - it's not - what I learned - it especially got another dynamic when Clio [Montrey, social media strategist] came in. I learned that social media is much more about being social than doing things online within an anonymous online community. This is something which I didn't expect to happen at all, which is a really beautiful surprise in a way. I get reactions which are really very warm and very, very encouraging. And people really - like this one woman who wrote to us 'It took me a lot of courage, but now I really want to tell you this is something absolutely special what you are having going on." (Team discussion on 4 th of October 2014).

My colleagues would have preferred to see their task in producing 'a good work of art,' not in interacting with what they called 'anonymous onlookers.' They confessed that they did not believe in the worth and authenticity of communication which is carried on online. Their primary goal was to create an artwork that reflected their individual identities, rather than ensuring the selfdetermination of our community, or pursuing the general curiosity inherent in a research setting. Moreover, the attempt to engage with an anonymous community on creative matters, and indeed on occasion personal and artistic 
matters, via social media felt too much like an invasive challenge to their own artistic confidence. To them it felt like an intrusion to give the online community insight into our process of creation, whereas I wanted to make the process of making art and what it means to be an artist more transparent to the community.

Bernhard Hütten: "You know I felt a bit overwhelmed knowing that this would suddenly be a bigger part in the project than I thought. And I went along with it and ok, you know, let's give it a try, but for me it's still difficult to engage with the blog."

(Team discussion on $4^{\text {th }}$ of October 2014)

They couldn't relate to the blog, it did not interest them as artists, and they felt it took away from the artwork. Consequently, they were not willing to promote any TransCoding material on their personal social media sites, which they usually used as a means for personal or artistic promotion only.

Bernhard Hütten: "I look at the posts and some of the posts I find relevant, but I cannot engage with it at all, it is not something I am interested in."

Connor Willing: "You are reluctant to see the work that we are making together as the team, if you like, in the same context as the blog, because you feel the blog is taking away from it a little. But maybe this is our challenge, you see. How do we make the blog, or how do we, you know..."

Bernhard Hütten: “...integrate it.” (Team discussion on 4 th of October 2014)

My colleagues proposed to reverse the process of communication by creating the artwork first and only afterwards trying to engage the community. They believed that the artwork should inform the blog, not the blog the artwork.

Connor Willing: "You know I sort of feel, if we had - if the blog had come a little bit later, then the work we would have had established would start to lead the blog maybe a bit more and that may have been the main content of the blog. Whereas at the moment the blog is full of all these different, you know, things that may or may not relate directly." (Team discussion on $4^{\text {th }}$ of October 2014)

To make art and at the same time to go online with it didn't prove to be a safe environment for my colleagues and for their way of doing art, even after they had given permission for certain content to be posted online. Even though they had consented to participating in active online interaction, they nevertheless felt uncomfortable.

Anna Veenen: "For me one of the things that is problematic with the blog is - as you earlier said - the safe space. That was really for me quite difficult ... You know these videos [which showed her trying out some interactive gestures - author's note] are really just for us or for 
me. I don't want to be pushed to say 'no' or 'ok,' or to think 'is it so bad if this is online?'” (Team discussion on $4^{\text {th }}$ of October 2014)

Showing the artwork at a too early stage felt like a risk to them as professional artists: they didn't want to be associated with anything that in their opinion was not 'perfect' yet. The same applied for integrating contributions that possibly didn't meet their demand for quality. Not only was it a question of taste to my colleagues, but it also got in the way of their habit of collecting the materials themselves and having total control over them. Moreover, they were afraid that if the material was 'not good enough,' it could reflect badly on them as serious artists and could damage their reputation in their new music peer group.

We realised that the structure of the social media, the building of a community and the speed with which social media works was too different, for some of our group, from their working habits and their wish for depth. To work within a participatory context demanded continuous engagement with a community that was perceived as 'anonymous' by my fellow artists and as getting in the way of meaningful collaboration within the group of artists they had originally wanted to work with. The openness that my social media strategist and I felt was necessary to gain the trust of a community and to get the community to interact with the arts team was opposed to the need for a safe haven in which the artists could build their artwork.

Although we had set strict privacy rules for ourselves, the feeling prevailed of not being allowed to 'fail and fail again' in order to develop the artwork, because the failing could possibly become part of the next blog post for the category 'Making of.'

At this point I would like to return to Jank's thoughts on participation as paraphrased in chapter 4 ('Theory of Participatory Culture'). The development of What if we had wings? and its participatory aspect clearly raised 'questions of power constellations' and of the 'congenial inclusion of outsiders.' It enabled 'open, critical dialogue' not only with the community but also among the team, as well as the dissolution of traditional practices (Jank 2012: 147). We understood that we needed to develop a new mode of operating; we had to search for a way to include contributions from our community in a meaningful and responsible way; we had to make the border between 'us' and 'them' the 'outsiders' - permeable; we had to be willing to be influenced and had to value the community. We had to give up some of our control over the artistic material and instead value our community members' creativity and integrate their contributions. We needed to be willing to hear the voice of the community and to share power over the vision for the overall artwork. All of this had to be accomplished without losing ourselves or our artistic integrity.

However, since my colleagues could not find additional value in the participatory 
setting for themselves and for their art making, since they found it got in the way of what they really wanted to do, we decided to end the collaboration after the first year of TransCoding. There were a number of valid points that had rather to be consciously acknowledged than suppressed: fear of losing the respect of one's peer group; fear that one might not fit in anymore; discomfort in handling material that is not one's own, not to one's taste or maybe not of the quality one is used to working with; unease with operating beyond our self-chosen and established artistic 'brand image'; the need to feel safe in one's working process; a familiarity with well tested and comfortable working habits that made one resistant to an as yet uncertain approach. The pressure from the domain we are working in and from the field that an artist answers to cannot be denied, neither can (our personal) artistic taste be too much ignored in the working process. Both are factors in our professional life as artists that cannot easily be changed.

I would therefore like to look more closely at an example in which a (subliminal) friction between a community member and myself occurred, in which a conflict of 'taste' versus 'authority' came to the surface and in which I was challenged to overcome several of above mentioned points. The perspectives I describe in detail include both that of the artist and of the individual community member.

\section{Read me - Personalised for Maria Hippenfels: A Conflict of 'Taste' Versus 'Authority' With a Positive Outcome}

We always heard from the installation's subjects that the personalised installations were meaningful to them. I quote Maria's representative email to me (February 2, 2016) about the music I composed for her soundtrack:

A)) Link to the soundtrack Maria - a Portrait:

http://transcoding.info/english/book.html - PART II CHAPTER 7 (6)

„Liebe Barbara!

Ich hab's mir gestern schon angehört und wollte es noch einmal nachklingen lassen. Dein Soundtrackversuch ist unglaublich. Und selbst dieses Wort kann gar nicht gut genug ausdrücken, was ich empfinde. Ich bin sehr berührt und ich habe das Gefühl, dass die Musik ganz tief in mich hineingeht. Ich kann mich zu 100\% damit identifizieren.

Ja! Es ist GENIAL! Und ich bin so unsagbar froh, dass ich ehrlich war zu dir und darauf gewartet habe, dass du Zeit dafür hast.

DANKE schon einmal.

Also ich würde gar nichts ändern. Mein einziges Problem dabei ist, dass ich nicht genug davon bekommen kann. Es ist mir immer wieder zu kurz und von mir aus, kann es ewig andauern. ;-) 
Sag mir bitte doch noch einmal, was du von mir brauchst [bezieht sich auf Textmaterial für die Installation, A.d. Verf] !

ganz liebe Grüsse,

Maria"

(Maria in an email to the author on February 2, 2016)

"Dear Barbara!

I listened to it yesterday and I wanted to let it sink in for a while. Your soundtrack attempt is unbelievable. Even these words can't sufficiently express what I feel. I am truly moved and have the feeling that the music touches something deep inside me. I can identify with it $100 \%$.

Yes! It is GENIUS! And I don't have words to tell you how glad I am that I was true to you and waited for you to find the time to compose it.

THANK YOU already in advance.

So I wouldn't change a single thing. My only problem is that I can't get enough of it. I just always feel it is too short, and as far as I'm concerned, it could last for ever. ;-)

Please tell me again what you need now [with regard to the text material for the installation - author's note]!

All the best,

Maria"

(ibid; translation by the author)

Reading the exuberant email from Maria, one could think that she was trustingly relinquishing all authority over the music and the installation to me and that she was following me blindly (but happily) in whatever I offered. That was not the case. I would like now to explore the question of who had authority in which part of the process and where friction arose.

Maria Hippenfels is a dance and kindergarten pedagogue. She teaches elementary music education and holds drumming workshops for nonprofessional musicians. I had met Maria in the environment of "Schmiede Hallein," a place for creative people who want to collaborate for ten days and create something out of the situation. When Maria approached me for her version of Read me, I was a little hesitant. I had heard her sing and improvise in the room next to me. Her improvisations gave me the impression that her creative taste was significantly different from mine, maybe too much so for me to handle easily in the context of the installation. However, Maria repeatedly approached me and almost urged me to personalise Read me for her. In the end I took on the challenge, not wanting to disappoint her.

For her installation she gave me a spiritual poem by Hermann Hesse, and a text paragraph on rhythm from a description of a film she loved. In her selfassessment she described herself as a person of contrasting facets: rhythmic, 
very active, but also very soft and melancholic. We recorded her improvising on the djembe and with her voice. At first this happened without any guidance from me: she improvised the way she wanted to. Later, I asked her to experiment with sounds and to try extended techniques on her instrument, hoping to get material closer to what I usually use. Subsequently, I sorted through the material and started to compose with it, trying to combine the information and material I had into a coherent piece of music that would describe her. I soon realised that I was having difficulty finding a bridge to link her sound and text worlds with mine, and that I was struggling not only on an artistic level but also on an emotional level to merge the disparate information I had. After a series of fruitless trials I asked Maria if I could delegate the composition to my colleague Clio Montrey, who is very experienced in the crossover between high art and popular culture, and who I thought might meet Maria's expectations and wishes more easily than I felt I could.

Maria replied that she could not identify with Clio's art as much as she could with mine and that she would rather put up with a longer waiting period to get a soundtrack composed by myself. In consequence that meant that I needed to find a way into her world without entirely losing my grip on my own. I realised that I had to get rid of whatever input had come from me. The improvisation I had guided her in was not representative of Maria; the way I wanted Maria to appear was not the person she was. Therefore, I had to find a way to relate to her by repeatedly going through her material and finding out what to me was the essence she wanted to convey. Only then I could add sounds of my own to her djembe playing and her singing and render the whole into something I hoped would capture Maria as a person.

In the end, Maria and I were both content, although I am aware that the soundtrack for Maria is not a soundtrack I would usually compose for myself, since neither the music nor the text Maria chose was entirely to my taste. Nevertheless, it satisfied me that she felt I had understood her through my art and that I had found a way to express her personality using her given material. In her persistence that she wanted this installation, and in her not yielding when I wanted to delegate the compositional process to somebody else, Maria fully exercised the authority the concept of Read me gives her. She challenged me to incorporate her ways of expressing herself into my art in a way that was meaningful for both. She changed the traditional (power) model of the artistaudience relationship - in which the artist is powerful and creates, whereas the audience (reverently) consumes - into one of equality. Artist and audience listen to each other and co-create.

In the next section I would like to touch on another phenomenon we encountered with this project. The community takes over and makes the material their own. 


\section{SoundCloud - Unlimited Authority Generates Additional Value}

We granted unlimited authority to our community members when we shared our artwork or our sound material with them under a Creative Commons license. This effectively meant that we left it to the community's creative discretion how to use our material, and we occasionally encouraged them to exploit it for their own artwork.

We considered this as part of meeting our audience within an 'engagementbased' versus 'appointment-based' model. I draw this terminology from media scholar Henry Jenkins et al. in their book Spreadable media: Creating value and meaning in a networked culture. (Jenkins, Ford and Green, Spreadable media: Creating Value and Meaning in a Networked Culture 2013) In TransCoding I define the 'appointment-based' model as the one where artist and audience/ community meet each other in concerts without any deeper interaction, whereas an 'engagement-based' model means that the community not only meets the artist and consumes what art is offered but engages in a meaningful way through sharing, exchanging and creating additional value.

Our SoundCloud (https://soundcloud.com/what-ifblog/) was the most obvious channel on which we could offer them the opportunity to interact with us in this particular way. If people used sounds that we offered for free use under a Creative Commons license, we asked for credit and a link back to us, but had no control over it. Without being actively encouraged by us to do so, members started to use and remix the contributions partly in contexts different from ours (Fig. 2.24: Andy Getch February Album Writing Month, FAWM). 


\section{FШF

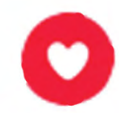

FWF

liked your Tweet.

\section{View}

what-ifblog.net @what_ifblog

Community member @AndyGetch1 has used our sound samples from our @SoundCloud to compose a @FAWM track! Listen here: fawm.org/songs/58611

Fig. 2.24: Tweet from 15th of February 2016 about community member Andy Getch using sound samples from the what-if-SoundCloud in a context other than TransCoding. Image (C) TransCoding 
Our SoundCloud stats show 336 downloads of our material in 2015 and 2016. We once even made a double loop: Community member Gloria Guns used our sounds for her remix Fan Death and I used her remix as a sound pool to personalise Read me for her:

- Link to the video documentation of Read me personalised for Gloria Guns

(1)) Link to the remix Fan Death by Gloria Guns:

http://transcoding.info/english/book.html - PART II CHAPTER 7 (2)

The community began to use not only the material we provided, but also materials that they provided to each other, and get in touch with each other independently from us. We considered this as a value in itself. They worked as what anthropologist Grant McCracken calls 'multipliers' in his essay "Consumers" or "Multipliers".

"A 'multiplier' is someone who will treat the good, service or experience as a starting point. Multipliers will build in some of their own intelligence and imagination. They will take possession of a cultural artifact and make it more detailed, more contextually responsive, more culturally nuanced, and lest we forget the point of the exercise, more valuable. Using a term like 'multiplier' will help the meaning maker keep new realities front and center. If there's nothing in the product, service, or experience that can be built on, well, then it's back to the drawing board." (McCracken, Spreadable Media 2013)

Besides sharing and passing along content, liking, commenting and contributing, our users independently generated value by and for themselves, showing the engagement of an active audience. The status of our members changed from consumers to independent makers to multipliers.

\section{A Potential for Change}

In their paper Mapping of practices in the EU Member States on Participatory governance of cultural heritage Sani et al. ask "What is the point of the public's participation? ... [T] his question has become one of the key questions in the debates about participation and the evaluation of participatory practice and it defines key trends and questions that concern those engaged in developing participatory practice." (Sani, et al. 2015, 68)

They claim that each project should investigate what kind of difference the participation practice offers in people's lives: whether it is an active agency a resource for capability development and self-determination, or if it offers potential for organisational change (in our case, change in the production of art and in the power relationship between artist and audience). Additionally, they 
ask if the project shows a commitment to dialogue, debate and reflection with all stakeholders. (Sani, et al. 2015)

In TransCoding we strove to change the traditional (power) models of the artist-audience relationship through participatory culture. An indicator for a successful power change is, if the notion of the artist as lone creative genius and the audience as mere spectators and consumers changes into one of mutual influencers, inspirers and co-creators. Both parties are in a dialogue, listen to each other and get to know each other. The community participates in the creative decision-making process, in the creation and the reflection of it; the artist works in dialogue with the community instead of alone.

The activity of the community moves from mere consuming, and "using and choosing" art to "making and shaping" (Cornwall und Gaventa 2001) by establishing a creative communication and partnership with the arts team and by developing their own creative capabilities and determinations. How are these values reflected in our project?

\section{Commitment to Dialogue, Debate and Reflection}

In TransCoding it is not the artwork, but rather the dialogue with our community that first comes to mind. They are at the foreground of the team's thinking. Before even starting to create art, we think about our community and what could interest them. We begin conversations for the purpose of learning what triggers the imagination of our members, what captures their interest and what gets their creativity going.

We communicate as directly as possible, exchanging with the community via blog posts, music, images, chats, email or comments on our social media platforms. We listen to their words or music and feel a responsibility to acknowledge their ideas and wishes, reflecting them verifiably in our artwork. Community members can express themselves via their creative contributions, guest blog posts, through interviews or feature posts, comments or likes. They can feedback negatively - if they choose to - by ignoring our triggers or in comments. All stakeholders have a voice before, during and after the creation of our joint artwork when joining the dialogue, debate and reflection on our social media channels.

About the change in the aesthetic approach and outcome seen from my perspective as the lead artist, I claim that thinking about our target group while developing Slices of Life, listening to the sounds they contributed, looking at the images they sent us and the text or thoughts they provided, effectuated my design of the evolving artwork. With TransCoding new music or multimedia art could not 'hide' behind abstract beauty; instead, the work needed to have connotations to which the community could relate, and in which their influence showed. 
Various strong elements of concreteness permeate the often-found abstractness of European avant-garde music I originally come from: I worked with a string of stories that feature identity, related to the lives of our community members and picked up the stories they contributed; I incorporated images and texts as originally submitted, without manipulations; I created a sound language that was relatively clear although rooted in extended instrumental techniques and electronic music; I didn't hesitate to use sound design to underline and interpret texts, thoughts, images or identities the community entrusted to us. The direct communication with my audience and their works inspired and influenced me, while all the time demanding a large degree of flexibility.

Here, a clear indication of change was that I altered my aesthetic decisionmaking and the content of the artwork through my exchanges with the community and their contributions. The (non-professional) community members experienced and expressed personal change and added value through the recognition of their artwork in a different (professional) environment. They gained additional reputation, since an internationally renowned artist performed their (clearly designated) contribution at prestigious festivals, and their personal voice is now heard and appreciated by an international expert community. As an example here are a few responses when I announced a talk on the project on Facebook. The comments indicate the significance of the project for individual community members:

Sabina Ulubeanu: "Honoured to be part of it! Good luck! $><$ "

Anahit Mughnetsyan: "really big honore for me too guys!! greetings from Armenia !!! and big good of luck from our side ! :- :)"

Laura Feathers: "I'm honored to be a part of your beautiful project! :)"

Ricardo Tovar Mateus: "I wish you all the best, im sure the talk will be very inspiring like you and the project are Barbara $\because *$ "

Alina Murzakhanova: "Dear Barbara Lueneburg, it was a very interesting and meaningful project, you have invited me to take part in! I wish you to continue this way and show to the audience more and more wonderful artworks!"

(Feathers, et al. 2016)

\section{Organisational Change in the Production of the Artwork with Relation to the Community}

As mentioned above, on the blog I revealed parts of my personality, tried to show how I work or how I think about art. In this way I challenged or 'humanised' the notion of a 'creative genius' to a level that is more tangible, thus consciously altering the traditional power relationship between artist and community. Actively contributing community members likewise shared in the personal involvement 
I brought into the project. Personal interactions with the TransCoding team even when happening online - appeared to assist our community members in overcoming their inhibitions in experiencing new music and new media art. In chats or interviews some members told us that they ventured into areas of art that they had previously not explored.

Likewise, the interaction with the community helped me to critically question my cultural socialisation, the art scene I am working in, and my artistic background. I consciously looked for tools to make the art I develop for and with our community accessible without renouncing my personal context and cultural heritage. My peer group in the contemporary (art) music field has begun to take notice with invitations to festivals in Germany, Canada and Austria and comments on performances such as "the epic Slice of Life" (Canadian Music Center 2017) or "probably the most progressive practice currently taking place in music and media composition." (Flüh, Neue Musik von der Urbevölkerung und der Blog-Community - Barbara Lüneburg bei und das Abschlusskonzert mit dem DSO von Ultraschall 2016 2016)

\section{Capability Development and Self-determination for Community Members through Participation}

In our online, social media community we observed change on many more levels: in the self-confidence of individual members; in an increased motivation to create; in trust and openness towards the team, indicating a change in the traditional (power) models; in pride in being involved; in pride in being acknowledged as contributor to the artwork; and in the interest in sharing a discourse. My artistic ideas on the other hand were influenced by and developed through the communication and debate with and through the work of the participants and their reflection on the project.

Community members appreciated the personal contact with us. They sometimes gave critical feedback or asked for advice. Anahit Mughnetsyan from Armenia, for instance, chatted with me on Facebook and offered critical thoughts on our Call for Entries: Catrina (http://what-ifblog.net/2014/10/27/ call-for-entries-catrinas/)

"but your comunity [sic!] gallery is full of strange i think articles ....sorry who is catrina? ... much negative energy comes from ... you think that my article is ok next to them dear Barbara?” (Anahit Mughnetsyan chats with the author on Facebook, March 1, 2015)

A few days later Anahit asks for feedback on her work.

"dear Barbara sending to you scores i wrote but as you know i am not composer just creations of me see and say pls honestly how are they ..." (Anahit Mughnetsyan, Armenia, on Facebook, March 6, 2015) 


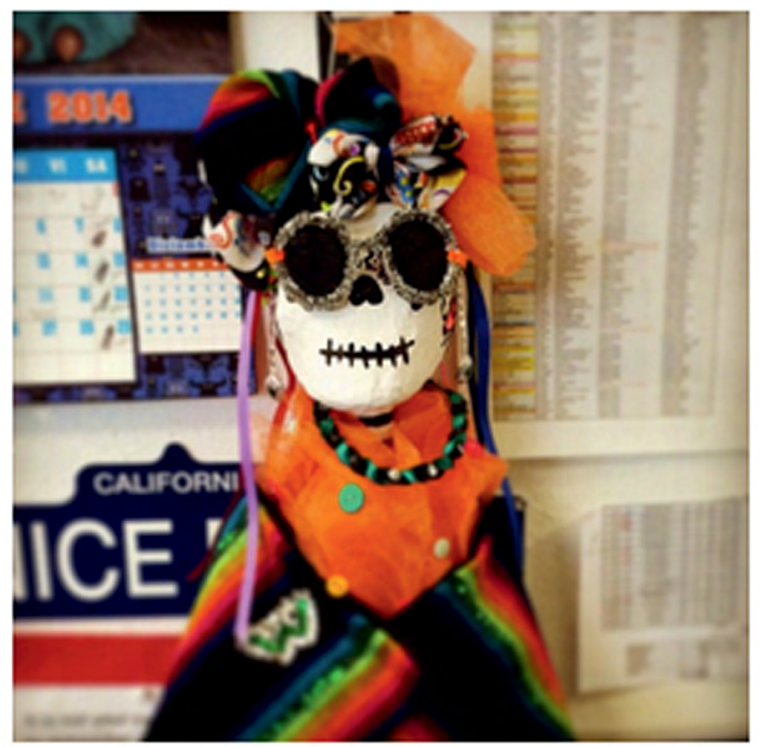

\section{Catrinas | Submissions}

\section{Rounding out our call for Catrinas is this lovely submission from Eline Fris and her coworkers (Mexico City). They made this sweet Catrina doll at the office!}

Fig. 2.25: Screenshot from one of the 'Catrina' submissions that Anahit disliked on our Tumblr community gallery. We received the image in response to our call for entries on the occasion of the Mexican national holiday "Day of the Dead." Image (C) TransCoding

Being included in our artwork and possibly featured in big festivals and at international venues gives motivation to participate, carries a fascination and is a reason for our community members to be proud. Gloria tweeted about her personalised version of Read me on her personal Twitter account @gloooooria on February 12, 2016:

"when the artist becomes the art ... an installation about ME by @e_violin of@what_ifblog: youtube.com/watch?v=ZkJTh3 ...” (Gloria Guns on Twitter, February 12, 2016) 
The actual activity of writing, photographing, composing and thinking about our challenges holds in itself joy and fascination. Anthony Green comments on the project's Facebook page, February 22, 2016: "Thank you for this awesome project!" (Fig. 2.26).

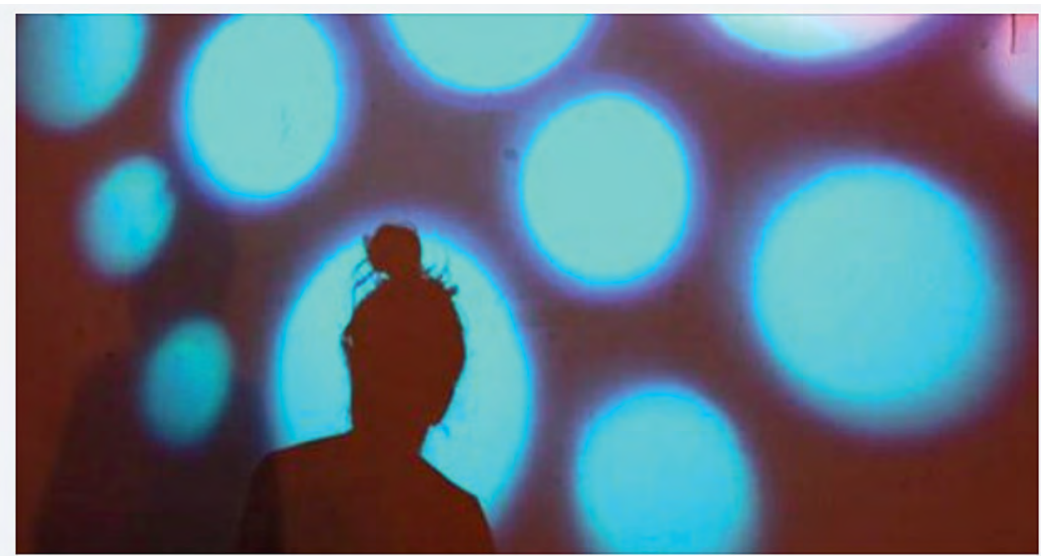

FEB Call for Entries | \#GYHAFY

16 Feb 16, 2016 - Feb 29, 2016 · worldwide

12 Went $\cdot 8$ Interested

Like - Reply · Remove Preview · $2 y$

What if? A participatory arts project Anthony Green's transgender male has come in. (Thank you!) He lives in the 80th century. From Intorion (transliterated). Have a read.

(b) 1

Like $\cdot$ Reply $\cdot 2 y$

Anthony Green Thank you for this awesome project!

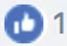

Fig. 2.26: Anthony Green just added a lengthy contribution to our Call for Entries I \#GYHAFY - Take A Holiday Away From Yourself and thanks us now "for this awesome project." (Facebook, February 22, 2016)

During offline workshops in the framework of TransCoding another indicator for a successful power change in the artist-audience relationship became tangible. Here the authority in decision-making with regard to content, aesthetics, form and production of the individual or group artwork lay with the participants alone. The participants used the framework of TransCoding as an 'active agency- 
a resource for capability development and self-determination' as Sani et al call it. Last but not least, I would like to quote my co-worker Clio Montrey, who also experienced personal change, capability development and self-determination through the project. The example demonstrates the difference participation in TransCoding was able to offer in people's life.

“Today I submitted a manuscript to a literary competition. No matter the outcome, I feel like I've already won a tremendous prize.

In early 2015 I was still too shy about my creative writing to share a single piece of fiction online or in public ... I've been steadily writing fiction since I was fifteen, including a very questionable early steampunk novel (it did have dragons, though) and various short stories over the years. It was always for myself ...

But just over a year ago, I could no longer bear it. I decided it was time to start sharing. I began posting tiny snippets of my novel-in-progress along with the SoundCloud tracks I was composing. In July I finally posted a story on my blog. In October I had the pleasure of reading at Resonance (thx Klara!) [Klara Plessis, poet from Canada]. Now I'm here. A few days ago I rediscovered a novella I'd begun in 2012. I've since begun working on it again while allowing my "big novel" a rest before I do a new draft. I'm taking my time but also exploring exciting new areas of creativity I didn’t even know I possessed.

Thanks for reading and commenting and giving me feedback on the short pieces of flash fiction I've shared so far! Thanks to Barbara [Lüneburg from the TransCoding team] for getting me so involved in blogging that I could no longer deny my deep love for writing. And thanks to everyone who read this post!

Much love, Clio"

(Status from March 25, 2016, on Clio Em's personal Facebook site)

Working in the context of TransCoding afforded Montrey community (and professional) approval, encouragement and a field of practice for her writing which resulted in a different self-perception of her creative abilities.

The following chapter discusses whether we can gain new scholarly insights through arts practice within the specific framework of artistic research. We compare 'knowledge gain' and 'methodology' in 'artistic research' and 'ethnographic sociology.' For this investigation I collaborated with my co-worker Kai Ginkel, who contributed to the chapter from his expertise as a sociologist. 



\title{
III. ARTISTIC RESEARCH - NEW INSIGHTS ThroUgh ARTS PRACTICE?
}

Barbara Lüneburg, Kai Ginkel

\begin{abstract}
"The history of a concept is ... that of its various fields of constitution and validity, that of its successive rules of use, that of the many theoretical contexts in which it developed and matured." (Foucault, Archaeology of Knowledge 1972: 4)
\end{abstract}

In the third part of this monograph, my co-worker Kai Ginkel - who comes from the field of ethnographic sociology and praxeology - and I as artistic researcher compare discourses in artistic research and ethnographic sociology with regard to theory and practice in each domain. In TransCoding ethnographic sociology and artistic research were employed side by side. Working together within the framework of TransCoding, Ginkel and I both found it inspiring and enriching to deal with the different methodological tools, approaches to interpretation, ways of representation and dos and don'ts of our academic home disciplines, and to debate their use for our research.

From our perspective, both artistic research and ethnographic sociology belong to domains in which - as Foucault puts it - "the questions of the human being, consciousness, origin, and the subject emerge, intersect, mingle, and separate off". (Foucault, Archaeology of Knowledge 1972: 16) We will compare the methodologies in both domains, and their data collection strategies, methods of analysis and evaluation, structures of fieldwork and connected power relations. We work under the philosophical paradigm of Foucauldian discourse analysis, searching for the origins of knowledge claims and trying to archaeologically uncover the construction of 'truth' and 'knowledge' with regard to the artwork I Am a Priest. We will examine the overall production of knowledge, while taking into account similarities and differences. Above all, we are interested in whether artistic research renders new insights through arts practice not only for academia but also for public audiences - insights that would remain inaccessible through other research methods. We will look at each discipline from the perspective of its particular specialist: for ethnographic sociology Kai Ginkel, and for artistic research myself. 


\section{Framing the Study}

In chapter 10 ('Artistic Research and Ethnographic Sociology - One Project, Two Methods'), we first attempt definitions of artistic research and ethnographic sociology and offer rough outlines of practices, methodologies and research goals.

In chapter 11 ('Case Study I Am a Priest') we talk about my own artwork I Am a Priest for violin, electronics and video, which forms one segment of the multimedia show Slices of Life. I Am a Priest deals with the religion of Santería from the personal perspective of a practitioner. Ginkel and I will in turns investigate the artwork and the process of its creation from an ethnographic and an artistic research artistic perspective. We first recount where the origin of the artwork lies. We establish a series of events that led to the creation of I Am a Priest and concurrently served as a means for data collection with regard to Santería. We then follow the archaeology of the artwork and relate the different strata of research and creation to discursive practices in both artistic and ethnographic research, contrasting methodological practices and their respective outcomes. Questions with regard to knowledge claim and meaning making are at the basis of our analysis.

In chapter 12 ('A Comparison of Discourses') we juxtapose both domains' methodological approaches and principles in detail. We compare research set-ups, data collection, power strategies and values that stand behind the dos and don'ts of each discipline. We also closely look at the influence researchers have on their researched field, the researching subject and the domain. We consider the language researchers use to formulate results of both artistic and ethnographic-sociological research and compare the expressiveness, exploratory power and informative value different ways of presentation offer. In doing this we seek mutual enhancement and possible innovation in each discipline. 


\section{ARTISTIC RESEARCH AND EthNOGRAPHIC SOCIOLOGY - ONe Project, TWO MEthods}

In qualitative research, our philosophical stance, methodologies, the manner of data collection, questions asked and the audiences we aim for influence our claim to truth and how we are perceived by academic institutions and society. In his essay The Order of Discourse, Foucault states that

"in every society the production of discourse is at once controlled, selected, organized and re-distributed by a certain number of procedures whose role is to ward off its powers and dangers, to gain mastery over its chance events, to evade its ponderous, formidable materiality." (Foucault, The Order of Discourse 1981: 52)

Taking Foucault's thoughts as a starting basis, we examine methodology, aims and possible taboos of both disciplines, since we are convinced that in the exclusion of certain methods and approaches lie distinct clues to the values and the knowledge claim of an academic field.

\section{Definition and Methodologies of Artistic Research}

(Lüneburg)

The history of artistic research is a fairly recent one and also one of ongoing, as yet unfinished institutionalisation. Educational researcher Savin-Baden and Howell Major date art-based approaches emerging "from an educational event in 1993 at Standford University that was funded by the American Educational Research Association." (Savin-Baden und Howell Major 2013: 288) So relatively fresh is the genesis of artistic research - in comparison to academic disciplines such as sociology - that it is still subject to discussions among scholars as to how the field can be defined both from a theoretical and a methodological perspective. Savin-Baden and Howell Major describe the use of artistic research approaches as a means "to explore, understand and represent human action and experience.” (ibidem: 289) They observe that "researchers who work in the artsbased field are often seen as taking up an opportunity to challenge the dominant discourses ... in relation to the nature and purpose of research itself." (ibidem: 290)

There are different forms of artistic research with different methodologies. Some researchers divide them into practice-based and practice-led research indicating that the investigation either is based on arts practice or should lead to new knowledge for the arts. Savin-Baden and Howell Major suggest a differentiation between: 
"1. Arts-based inquiry: where the artistic process is used as research by artists, researchers and participants in order to understand the art itself or understand a phenomenon through the artistic process.

2. Arts-informed inquiry that is of two types:

a. where art is used to represent the findings of a study

b. where art is used to represent a response to the findings of an issue or situation studied. 3. Arts-informing inquiry: where art is used in order to evoke a response from an audience (in the broadest sense) made to a situation or issue: the response may or may not be capture." (Savin-Baden and Howell Major 2013: 299)

Artistic research operates from an inside perspective that is based on the immediate involvement of the artist in the process of art making and the practice within the field. Often the research object must first be created and the process of creation may be part of the investigation. Furthermore, the research is used to systematically study and possibly change the domain itself and the investigated field, including the artist him- or herself. Music therapists Austin and Forinash define artistic research as

"a research method in which the arts play a primary role in any or all of the steps of the research method. Art forms such as poetry, music, visual art, drama, dance are essential to the research process itself and central in formulating the research question, generating data, analyzing data, and presenting the research results." (Austin and Forinash 2005: 458)

This stands in stark contrast to traditional knowledge gain in academia which has been formed through concepts expressed in language, words and sentences. Accordingly, some academic faculties observe artistic research with reserve, scepticism or rejection unless - as philosopher and artistic research scholar Dieter Mersch states - it is validated through verbal discourse:

"der Erkenntnis- und Wissensbegriff [wird] diskursiv vorentschieden, so dass nur das Geltung besitzt, eine Aussage trifft oder ein 'Argument' formuliert, was die Eigenschaft von Sätzen besitzt. Wenn es folglich 'ästhetisches Wissen' gibt, muss es gleichsam immer schon durch die Sprache als privilegierte Form des Ausdrucks hindurchgegangen und in Sprache übersetzbar sein, oder aber es erscheint obskur, ein Missbrauch des Wortes, eine Anmaßung." (Mersch 2015)

"the notion of cognition and knowledge is discursively decided in advance, meaning that only that which possesses the characteristics of sentences is valid, is declarative or formulates an 'argument.' Accordingly, if there is 'aesthetic knowledge,' it needs to virtually pass through language as the privileged form of expression and it needs to be translatable into language; if not, it seems obscure, an abuse of the word, a presumption." [ibid. translation by the author] 
Conversely, there are representatives of artistic research for whom it is almost a taboo to make use of a verbal research report in artistic research. They argue that cognition, the search for 'truth' and new insights happen through the practice of art, that knowledge lies in and should be expressed through the artwork itself.

In a private talk and email exchange educational researcher Silke Kruse-Weber and I developed the following definition:

"Künstlerische Forschung operiert mit Formen des Wissens, die mit Methoden rein wissenschaftlicher Forschung alleine nicht fassbar sind. Dazu gehört das Wissen, das auf der künstlerischen Praxis basiert und durch und für die unmittelbare künstlerische Praxis gewonnen wird, sowie das Wissen, das sich in den Resultaten künstlerischer Praxis manifestiert und für dessen Vermittlung die Erfahrung des Kunstwerks notwendig ist. Künstlerische Forschung sucht nach alternativen Möglichkeiten, diese Formen des Wissens zu kommunizieren. ... Der gewichtige Unterschied der künstlerischen Forschung zur wissenschaftlichen Forschung ist, dass bei der künstlerischen Forschung die Ziele und Methoden zum Erkenntnisgewinn von Fragestellungen geprägt sind, die aus dem (strukturierten und reflektierten) unmittelbaren Involviertsein des Künstlers bzw. der Künstlerin in den künstlerischen Prozess und das künstlerische Werk entstehen." (KruseWeber and Lüneburg 2016)

"Artistic research operates with forms of knowledge that one cannot investigate using scientific research methods alone. To this category belongs knowledge based on and gained through artistic practice, as well as the knowledge that manifests itself through the results of artistic practice for which experience with the artwork is essential. Artistic research strives for alternative possibilities to communicate these forms of knowledge. ... The essential difference between artistic and scientific research is that in artistic research, the goals and methods for acquiring knowledge are infused with the posing of questions that stem from the structured and reflective direct involvement of the artist in the process of creating the work and the artwork itself." [translation by Clio Montrey] (ibid.)

Another discussion among a group of artistic researchers of the University of Music and Performing Arts Graz (Austria) emphasises a method of knowledge gain through aesthetic experience that goes along with verbal reflection and includes the non-academic public.

"In den letzten Jahrzehnten hat sich eine Form der Forschung etabliert, deren zentrale Methode die künstlerische Praxis ist. Diese künstlerische Forschung eröffnet uns einen neuen Blick auf unsere Welt und ihre Phänomene. Durch ihren Fokus auf die Gestaltung ästhetischer Erfahrung und über das Hinterfragen künstlerischer Prozesse und ihres Kontextes, erlaubt künstlerische Forschung aus der unmittelbar erfahrenen Praxis heraus Strukturen offen zu legen, deren man sich mit anderen Methoden nur schwer gewahr werden kann. 
In der künstlerischen Forschung wird Erkenntnis durch sinnliche Erfahrung und verbale Reflexion mitgeteilt. Das Erleben der Kunst bzw. die ästhetische Erfahrung an sich ist dabei von zentraler Bedeutung für die Forschenden und deren Publikum, das so auch Teil des Forschungsprozesses werden kann." (Ciciliani, Eckel, et al. 2016)

"In recent decades a form of research has been established that is based on artistic practice as its central methodology. Artistic research affords us a new perspective on our world and its phenomena. Through its focus on the formation of aesthetic experience and through the examination of the artistic process and its context, artistic research allows us to lay open structures taken from directly experienced practice, which would be difficult to become aware of using other methods.

In artistic research, knowledge is communicated through sensory experience and verbal reflection. The experience of art, or more generally, the aesthetic experience, is of central importance to the researchers, as well as to the audience, which may also become a part of the research process." [translation by Clio Montrey] (ibid.)

Summing up the operational categories and characteristics of artistic research we can say that artistic research uses artistic practice as its central focal point. The research question arises from the arts practice and usually leads back to it. Typically, artist researchers first have to create the object or the situation they are investigating in a fieldwork setting: "[i]n the medium itself - in the creative process, the artwork and its effects - perspectives are revealed and constituted, horizons are shifted, and new distinctions are articulated." (Borgdorff 2012: 24) Knowledge is gained from an inside position. Consequently, artistic research requires its scholars to assume a high level of disclosure and maintain a critical and self-reflective position towards their practices, and the context they are placing their research in. The artist-researcher usually is a professional in the field he or she investigates and does not leave the field when the research is done, which may raise questions with regard to ethics and objectivity.

Artistic researchers claim that their research produces insights and knowledge that are more difficult, impossible even, to gain through any other method. In their opinion, the artistic-aesthetic experience holds knowledge that is communicated through the senses, the imagination and the mind. It evokes a response that reaches beyond verbally transmitted knowledge (as traditionally used in academic research). Consequently, in artistic research the representation of research results through the arts is of equal importance to the verbal presentation. Those representations can take the form of artistic artefacts, performances, or the creation of artistic situations or processes. Interpretation of these results may be more open than in traditional (qualitative) research. It democratises research, in the sense that audiences - other than those found in academia - might gain access to research knowledge through the experience of those artworks. 
"The specific nature of artistic research can be pinpointed in a way that it both cognitively and artistically articulates this revealment and constitution of the world, an articulation which is normative, affective, and expressive all at once - and which also, as it were, sets our moral, psychological, and social life into motion." (Borgdorff 2012: 24)

At the same time one could claim that the position of the artist is one of authority, since the (exploratory) power, informative value and expressiveness of a high quality artwork might very well guide the perception of an onlooker in a certain predetermined way.

In artistic research the philosophical stance of the researcher, methodology, data collection, data analysis and evaluation must be adjusted for each research project. Commonly adopted paradigms include phenomenology, pragmatism, post-modernism, post-structuralism, social constructionism and constructivism. A wide range of interdisciplinary approaches is followed, such as collaborations with researchers from sociology, psychology, ethnography, acoustics, musicology or other fields. Data generation and collection happens for instance via case studies, interviews, queries, and of course through the creative process itself, the resulting artwork and audience response.

Savin-Baden and Howell Major state that artistic research may use arts "for personal exploration of concern or issue," "to enhance understanding, reach multiple audiences and make findings accessible" or to evoke a response and "make meaning through complicated and messy performances and products that have power and are evocative." (Savin-Baden und Howell Major 2013: 299) I would add that researchers might explicitly aim for impact on the researched subject, their researched field and the domain itself.

\section{Definition and Methodologies of Ethnographic Sociology} (Ginkel)

The term 'ethnography' addresses a specific style of knowledge production in academic disciplines such as sociology, anthropology and political science. Ethnography "marks a distinction from quantitative approaches to social science and carries with it a commitment to a period and degree of immersion in the social setting being studied that is sufficient to reach a qualitative understanding of what happens there. Beyond this, ethnography can be put to the service of virtually any theoretical school: there are, for example, functionalist, structuralist, interactionist, Weberian and Marxist ethnographies.” (Shapiro 1994: 418)

Among the key virtues of ethnographic studies "is their focus upon the rich and varied 'real world' sociality recovered through a fieldworker's participation in the social life of some setting" (Hughes, Randall and Shapiro 1992: 124). Scholars applying ethnographic methodology strive to "produce detailed 
descriptions of the lived social experiences and social activities of social actors within specific contexts." (ibid.: 125) According to political and social science scholar Giampietro Gobo, 'doing ethnography' basically involves being present when things happen and seeing them with one's own eyes. Another striking feature "is the precision of the observations, the large number of details and the vividness of the account" (Gobo 2008: 4) According to John van Maanen, who wrote the highly influential Tales from the Field in the early 1980 s, doing ethnographic fieldwork requires "only a fieldworker, time, a bunch of people to talk with, and some writing materials," making it a research method that can be understood to be 'archaic' in terms of the basic technology involved. (Van Maanen 2011: 125) However, to Gobo, it is important "to understand that ethnography is a methodology (a style of thinking and doing), not a mere technique." (Gobo 2008: 15)

Ethnographic sociology first came to prominence through the seminal works by the Chicago School of symbolic interactionism in the first half of the 2oth century, when scholars such as Nels Anderson (1923, On Hobos and Homelessness) and Frederic M. Thrasher (1963 [1927], The Gang) would explore contemporary developments in US city life through participant observation, rather than by merely theorising about them from a distance. As Travers sums up "[t]he type of ethnographic research favoured by [interactionist scholars] Blumer and Prus requires a long period developing an 'intimate familiarity' with some group or social setting." (Travers 2001: 25) Consequently, in many cases practising ethnography means involving oneself in the everyday practices of a given field through actual participation. One's own involvement is being explored through means of observation, self-reflexivity and the subsequent ethnographic writing process, all of which serve the process of rendering the familiar strange (and vice versa) and objectifying the 'other.'

According to various sociologists, in ethnography the research process needs to allow for spontaneity while concurrently retaining a clear structure and traceability. Clifford Geertz, for instance, states that during fieldwork ethnographers face "a multiplicity of complex conceptual structures, many of them superimposed upon or knotted into one another, which are at once strange, irregular, and inexplicit, and which he must contrive somehow first to grasp and then to render." (Geertz 1973: 10) Giampietro Gobo suggests that the "researcher can select and follow a particular participant, letting his or her action define the boundaries of the context of observation" (Gobo and Molle 2008/17: 166), and Carol Cohn, researcher in the fields of gender, security and human rights, claims that ethnographic research can harbour both "preplanned" and "opportunistic" elements. (Cohn 2006, 94)

What does this look like in the various subdisciplines of sociological ethnography? Scholars from symbolic interactionism assume that for instance the 
meaning of objects or situations is not predetermined but instead established in actual interactions and might be modified in the unpredictable course of people's interactions. Interactionists do not assume that there is some sort of "hidden reality" behind what people do; instead, reality is brought forth by interactional practice. (Dellwing and Prus 2012: 21) Consequently, participation observation is the preferred tool in interactionist research.

Scholars coming from praxeology add a distinctively different perspective to the methodological palette. They focus on the body and materiality as well as on practical (often tacit) types of knowledge. (cf. Bueger 2014: 383) Meaning here is understood to be produced by a much wider variety of "doings and sayings" (cf. Schatzki 2002: 80) or, as Reckwitz adds, by "doing with things": "[N]ot only human beings participate in practices, but also non-human artefacts form components of practices. The things handled in a social practice must be treated as necessary components for a practice to be 'practiced'." (cf. Reckwitz 2002: 212) Here we see parallels to artistic research: researchers search for production of meaning not only through the process of creating art and the interactions that happen between, for example, artists and their audiences but also explicitly through non-human artefacts, the artworks.

In general, ethnographic scholars regard personal participation in the field as a way of having "access to meaning"; (Gobo 2008: p. 51) however, it is traditionally considered a taboo for a scholar to become too attached to their field (a phenomenon referred to as 'going native'). 'Going native' indicates that a scholar's personal involvement in the researched group hinders his or her reflective analytical work and is regarded a potential threat to the analytical process. (Hegner 2013: paragraph 14) Recently, however, modes of participation in which researchers get more deeply involved in the field are increasingly being discussed in ethnographic sociology. Matts Alvesson, for instance, speaks of the concept of 'at-home ethnography':

"a study ... in which the researcher-author describes a cultural setting to which $\mathrm{s} / \mathrm{he}$ has a natural "access" and in which $\mathrm{s} / \mathrm{he}$ is a natural participant, more or less on equal terms with other participants. The researcher works and/or lives in the setting and uses the experiences and the knowledge of and access to empirical material for research purposes." (Alvesson 2009: 159)

Gender studies scholar Jone Salomonsen's approach is based on the "method of compassion":

\footnotetext{
“'Compassion' ... does not refer to a wholesale positive embrace, nor to passionate criticisms and arguing, but to something in between: honesty. It shall designate an attitude in which belief is taken seriously, both cognitively and emotionally ... This means to leave behind the anthropological "method of pretention," which is mainly used to gain access, be it to rituals, to secret knowledge or to initiations." (Jone Salomonsen cited by Hegner 2004: 326)
} 
Both approaches deal with personal involvement and closeness to the researched subject - which is also relevant to artistic research - and they are regarded as fruitful and productive for the social sciences. However, Adler and Adler argue that with closer relationship and familiarity come specific responsibilities:

"Here we see people familiar with a setting having to create the space and character for their research role to emerge. They must look at the setting through a fresh perspective, to develop relationships with people they did not associate with previously, to change the nature of their preexisting relationships, and to become involved with the setting more broadly. This can be difficult, awkward, and heighten the sense of unnaturalness that invariably surrounds the research enterprise." (Adler and Adler 1987: 69 f.)

Therefore, even in cases where deep membership is considered acceptable, scholars always have to 'make the familiar strange' in order to be able to analyse and explore it systematically. (Coffey 2004: 21) A sociologist who himself practised so-called 'opportunistic complete membership' (Adler and Adler 1987, 78), Loïc Wacquant, warns that

"membership in a category or collective does not by itself make one a good anthropologist of it. At best it might make one an informant about it; at worst, it invites a descent into moral subjectivism, a parroting of the folk sociology of members, that is the negation of rigorous ethnography ... The warrant to study prizefighters, as any other social world, and by whatever method, comes not from the social ties that the inquirer entertains with members of that microcosm but from the theoretical problematic that animates the inquiry." (Wacquant 2005: 457 f.)

Overall, there are different approaches to the question of how to deal with the points of view and 'folk sociologies' of those participants commonly labelled as 'natives' by researchers in ethnography. We will later compare it to artistic research where 'being native' is an intrinsic part of the research.

Ethnographic research allows different forms of documentation for representing research results: social anthropologist Tim Ingold declares that ethnography quite literally means

"writing about the people. ... Ethnographic description, we might well say, is more an art than a science, but no less accurate or truthful for that. Like the Dutch painters of the seventeenth century, the European and American ethnographers of the twentieth could be said to have practiced an "art of describing" (Alpers 1983), albeit predominantly in words rather than in line and color. Theirs is still a standard against which we measure contemporary work." (Ingold 2014: 385) 
Gobo identifies a number of ethnographic approaches and writing styles applied until the 1980s, among them Erving Goffman's, who is considered one of the most influential scholars and field-workers in sociology. His writing style is known for its broad statements taken from detailed observation. However, he does not clarify which specific situation he bases his writing on. (cf. Gobo 2008: p. 43 f) Goffman does not introduce his participants as actual people or individual subjects with characteristics of their own; he does not write from a first-person perspective, nor does he make the specificities of the ethnographic process transparent. Instead, he focuses in a generalised fashion entirely on actions. Such a writing style may still be applied today, although some see it as outdated and even problematic. Ingold states:

"Much of this debate has fallen under the rubric of the so-called "crisis of representation." Quite reasonably, controversial questions have been asked about who has the right to describe, on what grounds any description may be taken to be more truthful or authoritative than any other, to what extent the presence of the author can or should be acknowledged within the text, and how the whole process of writing it might be made more collaborative." (Ingold 2014: 385)

Changes in presentation do not apply to writing styles only. Sociologist Sibylla Tinapp's photographic doctoral thesis on everyday life in Cuba, for instance, suggests that ethnographic research expands its methodology beyond that of being a purely pen-and-paper affair. (Tinapp 2005) Harvard University proposes a course in sensory ethnography offering its students "instructions in ethnographic media practices, producing a variety of original digital video, still photographic, hypermedia, and sound works." (Castaing-Taylor et al., Sensory Ethnography Lab: Harvard University 2010) and in her monograph Doing Sensory Ethnography Sarah Pink outlines this methodology as

"a way of thinking about and doing ethnography that takes as its starting point the multisensoriality of experience, perception, knowing and practice ... a process of doing ethnography that accounts for how this multisensoriality is integral both to the lives of people who participate in our research and to how we ethnographers practise our craft." (Pink 2009/2015: 1)

In this juxtaposition of some of each discipline's key features we find similarities between ethnographic sociology and artistic research, but we are also aware of the differences. We will continue by analysing the discursive practice behind the artwork I Am a Priest for violin, video and electronics while alternating between the perspective of artistic and ethnographic research. We reveal the archaeology of the piece by exploring various strata of its creation and meaning making, compare the processes of data collection, which kind of data are gathered 
and how it is done, and look into the analysis and evaluation of the data. Furthermore, we are interested in how results of the research are represented and communicated. 


\title{
11. Case Study I Am a Priest
}

\begin{abstract}
"The frontiers of a book are never clear-cut: beyond the title, the first lines, and the last full stop, beyond its internal configuration and its autonomous form, it is caught up in a system of references to other books, other texts, other sentences: it is a node within a network." (Foucault, Archaeology of Knowledge 1972: 23)
\end{abstract}

In this chapter, we aim to understand my artwork I Am a Priest via four different perspectives: firstly, through my experiences as a performer of the composition Toque a Eshu y Ochosi for singing violinist by Louis Aguirre, an artwork that is based on the religion Santería. Louis Aguirre, a Cuban composer, violinist and priest of the religion Santería, is a community member whose story is told in $I$ Am a Priest. Studying and performing his violin solo Toque served as one source of investigation for my study of Santería and consequently for the creation of $I$ Am a Priest. Secondly, we examine I Am a Priest through the "cultural resources and reference points" (Frogett, et al. 2014) I share with Louis Aguirre: my own personal background as a former practising Catholic, my participation in Shaman ceremonies, and our shared experience as violin students with Russian teachers. Thirdly, we investigate how identity is musically established and lastly, last but not least we look at I Am a Priest via the topic of spirituality, hereby establishing the context with regard to the community of TransCoding.

When I analyse the strata of I Am a Priest, I search for what Foucault calls "a system of references": texts, narratives and musical or personal associations the artwork might evoke and refer to, thus becoming a "node within a network." (Foucault, Archaeology of Knowledge 1972: 23) I am interested in what lies beyond, when we come from a material to an immaterial level, when art does something to us and we perform through it. Therefore, I set out to investigate the claim to knowledge that emerges from an artwork, even though I believe that this 'truth' is subject to change depending on the onlooker. When I present the artwork publicly, onlookers search for their own truth and for reflections of their personal experiences in it, possibly causing discontinuities between my intentions and their perception. Accordingly, I follow Margaret Wetherall as cited by Graham who states that

"discourse analysis said to be informed by Foucauldian or other poststructural theory endeavours to avoid the substitution of one 'truth' for another, recognising that "there can be no universal truths or absolute ethical positions [and hence] ... belief in social scientific investigation as a detached, historical, utopian, truth-seeking process becomes difficult to sustain." (Wetherall, 2001, 384 cited by Graham 2013: 114)

Correspondingly, Kai Ginkel approaches the artwork from another perspective, through the lens of ethnographic sociology. He introduces definitions and 
several main methodologies a researcher might use to get access to different manners of knowledge gain. In the ethnographic process, scholars may indulge in the method of participant observation, thus being able to experience social practice 'from within' instead of observing and analysing from a distance through the analysis of quantitative types of data. Throughout this participative process, research question have been developed that have often addressed topics of broad sociological interest, and making use of the fieldwork as a case study for a particular scholarly issue. Coming from a praxeological approach one might be interested in how religious practice is being embodied and what other types of materiality take on special meaning in the investigated field. Last but not least, in an analytical comparison of the 'foreign' culture and one's own, the research may shed light on and help to develop an increased sensibility for our own cultural surroundings.

\section{History of I Am a Priest and Data Collection: An Artistic Research Perspective (Lüneburg)}

Uncovering the strata of I Am a Priest means going back two years into my personal performance history, to a period before the actual creation of the composition. Its beginnings lie with my premiere of Cuban composer Louis Aguirre's violin solo Toque a Eshu y Ochosi for singing violinist on 24th of January 2016 at the festival Ultraschall in Berlin. It was my first encounter with the religion of Santería, which also served as data collection on this AfroCarribean religion. The solo Toque is an invocation of the gods of Santería. The performer functions as the priestess who conjures up the gods and plays and sings herself into an ecstasy.

Link to a video excerpt of Toque a Echu $y$ Ochosi by Louis Aguirre:

http://transcoding.info/english/book.html - PART III CHAPTER 11 (1)

Practising and performing Toque placed high demands on me as a performer. Not only was the violin part extremely difficult, but I had to concurrently sing a vocal part in Spanish, and at some point I had to accompany my own singing and violin playing with heavy rhythmical foot pounding. According to the composer, I literally embodied a priestess in a Santería ceremony.

The violin solo and the resulting concert situation are radical in the sense that the performer is exposed far beyond the norms of classical performance practice. Toque is a demanding and physical piece with an unusual expressiveness that reaches from guttural, grunted sounds to half screamed, almost sexual sounding passages to classically sung vocal parts with heavy vibrato.

Responding to my worries and questions when practising the vocal part, Aguirre wrote an explanatory email: 
"the vibrato,..just feel free, without thinking much, when it comes super,,,when not, just keep going..

...try as a trill first with the voice along...just to unleash the inner mechanism...and the feeling of something shaking/moving inside....

also, I think, the shake of your body while playing fast vibr. on the violin may help...use your body...get inside shaky trance...:-)

the voice will be beautiful no matter which type of voice the player has...

when we do rituals in order that it happens, reach Olofi (God in yoruba) certain things has to be said or chanted..or screamed..

you dont judge the voice of the priest.....it has to be there...so the magic happens...think it on that way...because is the way $i$ thought it...

we, in western culture, always focus on: do I like it? is it beautiful? but on these ceremonies no body will ask anybody, do you like it? the voice of the Obbá was 'beautiful'..(Obbá, name of a high priest in santería)...it has to be there, some prayers has to be sang, etc..

i say all that, to give you a bit of the idea behind...:-) and to easy your possible worries..." (Aguirre, Re: Solo 2013 [emphasis by the author])

As a vocally untrained instrumentalist I needed to overcome personal inhibitions to present the piece on stage. Aguirre declares Toque with its crossing of boundaries as an evocation of the deities through singing and playing; the bodily exertion experienced as a trance-like state, the fast and extreme vibrato as a religious possession - all signs that are typical for a Santería ceremony. He insists that the performance is not about aesthetic standards of beauty in classical Western performance. He claims that the magic of the ritual will happen through the performance and through the performer. The player doesn't just act out the role of a priest or priestess of Santería, he or she embodies it. [cf. emphasised text parts of quotation]

The process of practising and performing Toque can be understood as an arts-based inquiry. It was the first step of data collection, an artistic research process that offered me an entry into the phenomenon and practice of Santería. I gained data by way of immediate involvement and the means of embodiment. Through the preparation - several months of intensive practising - and the actual performance process, I gained an inside perspective on what worship in the religion of Santería literally might feel like; a perspective that encompassed mind, body, imagination and senses. Knowledge of Santería was likewise transmitted to the audience via verbal description, that is the programme notes, and via the audio and visual perception of the shared concert experience. During the performance, I was able to convey the physical experience in particular via mirror neurons.

"According to neuroscientist Viliayanur Ramachandran, when one person moves, mirror neurons in the brain of another person will react, 'as if the neuron is adopting the other 
person's point of view, almost as if it were performing a virtual reality simulation of the other person's action.' Mirror neurons allow us to empathise with other human beings and dissolve the barriers between people (Ramachandran 2010) and through their body language performers can control the flow of information and emotion in social interaction, and influence how the audience responds to their personality and feels their music. Nevertheless, the audience might reinterpret the leader's identification according to their values and their particular context." (Lüneburg 2013, 87 f.)

Moreover, Arnie Cox argues in his paper Embodying Music: Principles of the Mimetic Hypothesis that performances offer an invitation to take part and engage in the experience of what happens on stage via mimetic participation.

"When we take an aesthetic interest in something, whether people-watching or attending a sporting event or a film or a concert, our responses can be understood as if we are implicitly asking, What's it like to do that, along with the corollary question, What's it like to be that? Part of how we answer these questions is via MMI [Mimetic Motor Imagery], along with occasional overt mimetic motor action, as when we move to music in one way or another. In effect, it is as if we are responding to an invitation to somehow imitate and to thus take part. Accordingly, we can speak of the performing arts as offering a mimetic invitation, and we can speak of our various responses as mimetic engagement or mimetic participation, whether in the form of overt movement or in the privacy of covert imagery (MMI)." (Cox 2011)

Thus, a performance becomes a means to transport knowledge. In the case of Toque, I shared knowledge on the practice of Santería as I experienced it through my preparation for the concert and my actual performance on stage. In a second step, the performance of Toque was as a preparation for my own artwork in the framework of TransCoding: it served as a means of data collection for Slices of Life - I Am a Priest.

In this section I spoke about data collection through embodiment and how knowledge gained on Santería can be experienced by and transferred to the audience through arts practice. Next, Ginkel will analyse the data material of Toque a Eshu y Ochosi from a praxeological perspective. As Ginkel was not present for the performance of Toque a Eshu y Ochosi at Ultraschall Festival Berlin in 2016, his process of data collection did not include an actual observation of the situational performance of Louis Aguirre's Santería piece itself. Instead, his ethnographic perspective derives from his professional involvement in the proceedings of TransCoding as a whole. The data he could work with were notes from work meetings with me, the performer, written material such as snippets from interviews and email exchanges between composer Aguirre and myself and the programme notes on Toque he found on the website of Ultraschall Festival. 


\section{History of I Am a Priest and Data Collection: A Sociological Praxeological Perspective (Ginkel)}

On the occasion of the premiere of Toque by Barbara Lüneburg, Eckhard Weber wrote the following programme notes:

"Die heftige Klangsprache Aguirres, die in der afrokubanischen Priesterkultur gründet, führt das Publikum und die Performerin in extreme Welten. Barbara Lüneburg wird in Toque a Eshu y Ochosi zum 'totalen Instrument'. Stimme und Violine verschmelzen, Musik und innere Haltung werden voneinander untrennbar, wodurch sich die Szene gewissermaßen in eine rituelle, trance-artige Handlung verwandelt." (Weber 2016)

"The intense musical voice of Aguirre is based on an Afro-Cuban priest cult, and leads audience and performer into extreme worlds. In Toque a Eshu y Ochosi Barbara Lüneburg becomes the 'total instrument.' Voice and violin merge, music and inner stance become inseparable from each other, so that the scene effectively becomes a ritual, trance-like act." (Weber 2016; translation by Barbara Lüneburg)

Weber uses terms such as the German words 'heftig' (intense) and 'extrem' (extreme), words that suggest strong emotions. He implies that the experience of Toque will possibly lead both performer and audience into a world beyond the mundane, into a ritualistic, trance-like act. This echoes Aguirre's and Lüneburg's email correspondence, in which they talk about 'trance' and 'magic' when referring to the performance practice of Toque. The notion of emotional states that are understood as intense and extreme is echoed and specified in Louis Aguirre's own words. In his email, he gives Lüneburg helpful hints on how to use her body to understand how to play the piece.

From a praxeological perspective, the description in the first email paragraph quoted above helps to answer the question of how 'trance' and 'magic' are established through bodily and discursive activities. Instructing someone to 'unleash' some 'inner mechanism' and to 'get inside shaky trance' refers to this person's tacit (embodied) knowledge. We can only assume that Lüneburg as the performer understands what the composer would like to imply by it and that she knows how to translate this instruction into an act of competent bodily activity. As sociologist Stephen Turner suggests:

"Some activity, inference, or communicative act depends on both the user and the recipient possessing some inferential element or mechanism which allows them to understand, anticipate, co-operate, or co-ordinate with another. The typical sign of an element of tacit knowledge is that some people can perform the activity, including the activity of inferential reasoning, and others cannot." (Turner 2014: 155) 
Here, the performer's inherent body knowledge and practical skills translate a seemingly vague instruction into an artistic performance. To me - from my outside perspective - Aguirre's instruction would serve foremost as an indication of how performing the piece is particularly demanding or unusual in terms of physical activity. However, Aguirre specifies the role of the body for Lüneburg who grasps his meaning through context understanding and via tacit knowledge.

Trance and magic are established by an explicitly and specifically described bodily practice, as well as through the ritual of the concert and the shared acoustic and visual experience of violin, voice, performer and collective attendance. Vibrato, screaming and repetitive playing are conducive in establishing - rather than expressing - states of trance. 'Affect' is constructed by the bodily activity of the performer, the corporal identity of the instrument and the performer's body, the spatial and performative situation everybody is placed in and the ritualistic concert setting with all its connotations. In praxeological terms, 'affect' - as opposed to the everyday understanding of emotion - is established and shared among participants: "Affect is reminiscent of 'to affect' and 'to be affected' and thus of dynamic and interactive dimensions [emphasis by Ginkel] that the term 'emotion' lacks, as it rather implies the static notion of having an emotion 'deep inside'." (Reckwitz 2012: p. 250) Accordingly, we could state that the artistic practice in the concert situation and the experience of magic and trance is shared with the public via the dimension of 'affect.'

Furthermore, notions of 'magic' and 'trance' are made plausible through a distinction between aesthetics in the arts and in the religious ritual. Although Toque has been written for the concert hall, Aguirre understands his work as religious practice. The spiritual quality is established through a particular style of performance that, according to the composer, may defy conventional aesthetic rules.

\footnotetext{
"we, in western culture, always focus on: do I like it? is it beautiful? but on these ceremonies no body will ask anybody, do you like it? the voice of the Obbá was 'beautiful'..(Obbá, name of a high priest in santería)...it has to be there, some prayers has to be sang, etc.." (Aguirre, Re: Solo 2013)
}

The distinction is not made through abstract categorisation of what counts as 'beautiful' or 'not beautiful,' but through bodily performance. As Lüneburg wrote above, she needed to embody the role of a priestess of Santería through the performance and Aguirre claimed that by doing so the magic of the ritual would happen.

Establishing a state of 'trance' remains dependent on the performer's tacit skills that in this case draw on experiences that are common to both, Aguirre and Lüneburg. As Lüneburg outlines in the next section on cultural reference 
points, she and Aguirre share a history as violin students educated by Russian teachers. On several occasions she has participated in Shaman ceremonies and is thus familiar with rituals where the body is enacted in a ritualistic way. Both of these facts appear relevant to the indexicality of Aguirre's instructions and the way that she, the performer, understands the context to them. It also raises the question of whether the specificity of collaborating in performance practice may be dependent on shared personal histories or shared experiences.

To further understand the strata of I am a Priest, Lüneburg will next look into the "cultural resources and reference points" (Frogett, et al. 2014) she shares with Aguirre.

\section{Am a Priest - Cultural Reference Points: An Artistic Research Perspective (Lüneburg)}

After my premiere of Toque, I interviewed Louis Aguirre on his art and his inspirations. In a narrative interview setting he explained to me the role Santería plays in his art and life. Part of the interview constitutes the text that forms the basis of I Am a Priest.

"My music is devoted to worship my god.

I am a priest in the Santería. This religion came with the slave, is black people.

Ochosi is the hunting god and Echu is the god of the path. And then he is like a child. I mean he is the one that can close the path so nothing bad comes to you and he can open it, so good things happen to you.

First toque is for el Juan de la Juan tu.

The slaves were allowed to celebrate parties at the same day, in the wrong way of course, that the Catholics celebrate their own parties.

Cuba is a point of merging, a symbiosis between the Spanish and the African and a bit of the Chinese culture.

When people get possessed in some ceremonies you see them beating with knifes. They don't get caught, they walk in fire. It's unbelievable, it is a drive, a force of nature. You lose yourself, you are not yourself anymore. It is incredible, it is something that you are not you anymore. Entranced by the god. Because everyone is the son of some god, we believe that.

Echu Elegguá is like a child and is Jesus of Prague. St. Barbara is Changó and you have St. Lazarus that is Babalú Ayé. Echu Elegguá and Ochosi.

We have some gods that could be male and female and we don't know. We have this ambiguity. 
We give cigars and rum and whiskey to our gods, I mean alcohol, all that kind of things. It's, it's a weird thing, I mean, it's really."

(Aguirre and Lüneburg, Lyrics of the artwork „I Am a Priest“ 2016)

- Link to the video documentation of Slices of Life - I Am a Priest: http://transcoding.info/english/book.html - PART III CHAPTER 11 (1)

Aguirre's art is not only deeply rooted in the religion itself but also in his being a priest in Santería. In the interview he talks about Santería's relationship with Catholicism, describes some of the gods and goddesses, talks about how both his home country Cuba and Santería melt cultures and origins from different people. He describes trance-like conditions and powerful rituals. The Oxford Encyclopedia of African Thought: Abol-Impe, volume 1, confirms Aguirre's personal account, describing Santería as follows:

"A religion that developed out of the historical exigencies of the Atlantic slave trade in Cuba, Santería (Way of the Saints), also known as La Regla de Lukumi (Lukumi's Rule), is an AfroCaribbean religion created during the sixteenth century by enslaved Africans from presentday Nigeria and Benin, who mingled various Yoruba religious beliefs and practices with the Roman Catholic belief system of the Spanish plantation owners. Enslaved Africans from West Africa, imported to the Caribbean to work on sugar plantations, brought with them their own religious beliefs and practices, such as sacred drum music and dance, animal sacrifice, and the tradition of possession trance for communicating with ancestors and deities." (Irele and Jeyifo 2010: 305)

My personal history allowed me to relate to this. Santería shares rituals and personages with the Catholic Church; for example, offerings that are the focus of ceremonies, and saints who in the Santería version are re-interpreted as and reframed as their own deities. In I Am a Priest my own connection with the Catholic religion is mainly communicated through my choice of imagery of two different Virgin Marys that serve as symbols for the venerations of Santería gods. Powerful repeated violin motifs that first present and later answer the line of the Santería priest in a ritualistic form of call and response (the so-called 'lining out' that is common to both Santería and Christian rites) serve me as an artistic means to express Santería worship.

A)) Example for the use of 'lining out' in I Am a Priest: http://transcoding.info/english/book.html - PART III CHAPTER 11 (2)

I have witnessed and participated in Shaman ceremonies by an indigenous Canadian medicine woman and of a Mongolian shaman, whose ceremonies involved chanting, drumming and body rituals. Through these experiences 
I have an inkling of the power such rites can have over body and mind and the condition of trance that might be reached. My Catholic upbringing lets me relate to this as well, since I have experienced how rites in the Catholic Church speak in various ways to the senses. Through the use of incense (exciting the sense of smell), through call and response singing (speaking to the sense of hearing) or through the lighting and subsequent blessing of the Easter fire and the carrying of the blessed fire into the dark church space (stimulating eye and mind) Catholic priests evoke powerful rituals. In I Am a Priest trance and bodily possession are conveyed through the slow reverberant beatings of a bass drum, the human voice, screams and repetitive, passionate melodic motives.

Aguirre claimed that through my practising and performance of Toque I had a true experience of religious trance and connection with his gods, which he deduced from certain signs in my body in one of our rehearsals. He would argue that I had in a positive sense truly 'gone native' through an experience that I - at least partly - shared with my audience.

I understand my arts practice as performative, meaning that both the artwork and the creative process stir me (and my audience) and modify how we understand and reflect the world. I state that artistic practice can intrinsically have its own status in the search for knowledge. Although Loïc Wacquant might call my approach to participant observation and meaning making a "descent into moral subjectivism, a parroting of the folk sociology of members," (Wacquant 2005: 458) I prefer to follow Ingold, when he calls practising participant observation an education.

"But to practice participant observation is also to undergo an education. ... That is to say, it is a practice dedicated to what Kenelm Burridge (1975: 10) has called metanoia: 'an ongoing series of transformations each one of which alters the predicates of being." (Ingold 2014: 388)

The state of 'going native' - meaning that I personally experienced shamanistic rites when performing Toque - was an education that helped me to understand and later to artistically express various phenomena of Santería in I Am a Priest. 'Being native' through my Catholic upbringing, it was easier for me to understand the concept of Santería gods and goddesses that share predicates with Catholic saints. It also let me connect to the musical phenomenon mentioned above that forms part of a Santería service while having its roots in Christian psalm singing, the so-called 'lining out.'

Analysing the strata of I Am a Priest in search of meaning and epistemic potential, I searched for a system of references: texts, narratives and musical or personal associations the artwork might evoke and refer to. I claim that I can make an artistic statement on Santería that is valid in an artistic-scientific field, 
on account of my shared reference points and cultural resources with a priest of Santería and by practising and performing the religious ritual of Toque, that means by embodying certain aspects of Santería. Those become the references, the narratives, musical and personal associations that in the form of I Am a Priest grew into the "node within a network" of discourse and meaning. (Foucault, Archaeology of Knowledge 1972: 23)

Next, Ginkel investigates cultural references from a sociological perspective, approaching it from the opposite direction. For him, cultural reference points do not exist as such, but are constructed by people. Accordingly, he poses questions such as: How and by whom are rituals (i.e. cultural reference points) produced? How are they shaped as something which is convincingly powerful? And how does I Am a Priest fit into these considerations?

\section{Am a Priest - Cultural Reference Points: A Sociological Perspective (Ginkel)}

Louis Aguirre, the priest quoted in the artwork I Am a Priest, shares memories and narratives of his own experience in the practices of Santería. The snippets from the original interview that we read and hear are selected and rearranged by Lüneburg for her artwork. The recording has a casual and personal quality that displays Aguirre's deep knowledge of Santería. Through Lüneburg's artwork, Aguirre delivers a rather disjointed yet detailed account of spiritual practices. He talks about the history and context of Santería. He also describes specific situations experienced through physical activities in a Santería service. Aguirre labels those activities as remarkable, even unusual—or in the interviewee's own words, "weird." This particular word strikes me as interesting. From an outside perspective it could be understood as a judgement, while at the same time supporting our impression of his familiarity with Santería, which he presents in a playful manner.

Lüneburg offers a perspective that is slightly different, yet also rooted in personal experiences. She refers to reference points such as her Catholic upbringing, and to having experienced shamanistic ritual first-hand, although not in the context of Santería itself. Lüneburg reflects on her experience, remembering them as having a powerful effect on body and mind. She appears interested in the question of how shamanistic rites exert power over people, whereas sociology would ask how rituals are established as something which is convincingly powerful. Besides her being less directly involved in Santería rituals than Aguirre, there is another way in which Lüneburg's account differs from his. While the interviewee describes spiritual practice as a first-hand experience, Lüneburg chooses an account take from an encyclopaedia entry, adding scientific plausibility to what is presented both by herself and Aguirre with regard to 
Santería. Describing the history of this particular type of spirituality also allows her to establish links between an acknowledged narration stretching back numerous decades if not centuries in time and her own biographical history and ritual experience as we get to know them through her writing.

From a praxeological perspective we would say that Santería does not simply 'exist,' ready to be approached and observed by itself. Instead it exists as a bundle of practices we can see, hear, follow, observe and describe. It echoes what Lüneburg writes about Catholicism: “the believers' perceptive and emotional coding" is stimulated through "their senses of hearing (sermon, music, singing), smell (incense, candles), sight (clothing, architecture, illumination, paintings), and even touch (kneeling and [consuming] the host)" (Lüneburg 2013: 95) This is how Santería is established here - practically and discursively - as a network of practices, experiences, narrations, rearrangements, and historical accounts and this is how I Am a Priest becomes part of the practices it describes.

\section{Establishing 'Identity' through Artistic Means: An Artistic Research Perspective (Lüneburg)}

There is a third stratum of I Am a Priest that has to do with the overall artistic topic of the project, identity. Since the goal of the artistic research in TransCoding was to find artistic expressions of 'identity' in its many facets, I tried to capture a special aspect of identity in each section of Slices of Life. In the section of the artwork here studied, I Am a Priest, I accordingly expand the ethnographic investigation of Santería to an arts-based investigation that focuses on the representation of a person's identity epitomised through the personality of Louis Aguirre. His identity is characterised by his religion, and - as importantly - by his musicianship, when he works as a composer and former violinist.

Aguirre and I share personal reference points that can be found back in the artistic expressional means I use. We both studied violin with Russian teachers, he in Cuba and I in the former Soviet Union. During the interview we talked about how being taught by them was more than an instrumental education. It was an encounter with artists who showed a deep passion for life and for art that holistically formed both our personalities as artists and later as teachers.

Our shared experience left its mark in the way I treat the violin in I Am a Priest. The part mainly draws from a classical sonority, virtuosic violin techniques and romantic gestures often played in octaves. It refers to Aguirre's deep knowledge and love for classical music. At the same time, its melodic structure relates to the singing of a Santería priest, which can be heard in the soundtrack, and reflects and represents religious practices. It is also used to convey passion and commitment, which seems to be a feature of both, of practitioners of Santería in general and of the individual practitioner Louis Aguirre. 
However, Aguirre and I share a space beyond the classical world. This is reflected in my occasional use of timbral means, extended techniques and abstract sound features of a sonoric language that is common to Western European contemporary art music and touches the art world Aguirre is a composer in. It shows for example when I musically describe the ambiguity of male or female gods ("We have some gods that could be male and female and we don't know. We have this ambiguity."). (Aguirre and Lüneburg 2016) I apply brittle tones, as well as sul ponticello techniques that touch on the overtone series and then abandon it again, and noisy, cracking scratchy sounds (sound sample 1). Nothing is absolutely clear, nothing is entirely in focus, the sonoric qualities waver.

A)) Audio samples showing the use of sonoric features typical for contemporary

art music in I Am a Priest (sound samples 1 and 2):

http://transcoding.info/english/book.html - PART III CHAPTER 11 (2)

I proceed similarly when Aguirre mentions that "everyone is the son of some god" and then lists the name of some of his gods: the violin prepares and highlights the textual enumeration through several bowed Es that flicker through timbres, maintaining variety and securing the interest of the listener, adding brightness and resplendence and, in general, serve as a plateau of concentration (sound sample 2). Thus, I musically underscore features of the gods by using elements and means of film sound design, the only difference being that I retrieve the sonic material from the world of classical contemporary music.

Documentary features such as the original tone of the interview, the material taken from the practice of Santería, and the ecstatic screams and shouts of a group of young people further emphasise the 'truthfulness' and authenticity we seem to experience in the (concert) situation. I further underscore the topic of spirituality by using a huge resonating bass drum that adds a steady, ritualistic beat (sound sample 3). Thus the musical material does what Nicholas Cook describes as a possible feature of musical multimedia in his book Analysing Musical Multimedia. It "transfers its own attributes to the story-line and to the product," "it creates coherence" and "participates in the construction of meaning" (Cook 1998/2000: 20f). Music adds to the credibility of the said scene and it confers meaning on a subconscious level.

\footnotetext{
A)) Example for the use of the ritualistic bass drum in I Am a Priest (sound sample 3): http://transcoding.info/english/book.html - PART III CHAPTER 11 (2)
}

Last but not least, I musically address the community of TransCoding by including electronically produced rhythm samples that are associated with popular culture rather than classical music (sound sample 4) thus alluding to their assumed (musical) identity. Later in the piece I merge these samples with the percussion instruments of Santería (sound sample 5). 
A)) Examples for the use of rhythmic sound samples taken from the world of popular culture that mix with the rhythmic features of the Santería practice (sound sample 4 and 5): http://transcoding.info/english/book.html - PART III CHAPTER 11 (3)

To quote Cook, the different styles used in the soundtrack and violin part of I Am a Priest "offer ... opportunities for communicating complex social or attitudinal messages instantaneously; one or two notes in a distinctive musical style are sufficient to target a specific social and demographic group and to associate a whole nexus of social and cultural values with a product." (Cook 1998/2000: 17). By intentionally applying different musical means and styles within one single segment I artistically establish identities that not only speak to different audiences but also mirror the symbiosis and merging of cultures that is inherent in Santería practice and Cuban everyday life.

When I chatted with Aguirre about I Am a Priest, he confirmed that I had caught the essence of the religion.

Barbara Lüneburg: FB chat with Louis Aguirre, Apr 17th, 10:36pm

“Did you really listen to the new piece? [I am referring to Aguirre's 'like' on FB when I posted the video of I Am a Priest] To be honest, I didn't dare to send it to you. I didn't write your name to it, because I didn't want it to be too personal, but if you want, I would. Hope you are well, Louis. All the best, Barbara"

Aguirre: Apr 18th, 8:34pm

"Yes, I listened to it! I liked, and I think that you are doing something very particular. ... for me, it is a kind of composed 'Testimony' through a soundscape that includes the culture and 'soul' of the chosen 'subject.' many greetings, and hope you are doing fine too. L'

\section{Establishing 'Identity' through Artistic Means: A Sociological Research Perspective (Ginkel)}

Lüneburg refers to her compositional artwork as an expression of identity established with artistic means. However, sociologists often regard identity as established through social practices such as interaction, instead of seeing it as something constructed individually. Sociologists perceive 'personalities' as something that cannot and does not exist outside the realm of the social. Identity is established through interactional partners, in which the name of a person, their outward appearance and biographical data all play a major role. According to Goffman, identity is highly dependent upon the interaction in which it is presented and thus performed. (cf. Miebach 1991: p. 107)

Aguirre's identity here, 'performed' through both interview snippets and their musical embedding, is primarily focused on the priest-composer's spiritual 
life. The similarities in Lüneburg and Aguirre's lives as artists seem to form a backdrop that informs certain artistic decisions during the creative process. It is something that Lüneburg is consciously aware of and thus something that is an integral part of the eventual form of the piece. However, how do these biographical and artistic elements work in conjunction?

In the interview snippets Aguirre presents himself primarily as a Santería priest and therefore as an expert regarding ritual practice and the history of the field. Lüneburg picks up on these elements, amplifying and enhancing them artistically. She presents her own biography as in certain aspects similar to that of Aguirre. We find overlaps such as a similar education in music, a religious context that is in the widest sense at least partly based on Catholicism, spiritual shamanistic experiences and a passionate dedication to being an artist. Although in some aspects their biographies also show differences, Lüneburg emphasises their similarities. Additionally, she underpins Aguirre's historical explanations of Santería by quoting an encyclopaedia to confirm his statements.

On the artistic side, 'truthfulness' or plausibility is additionally established through 'technical' means. Lüneburg states that she uses sound design to underscore features of the gods. Seen from a praxeological point of view, this is how religious experience is often co-established or, at the very least, emphasised. Going back hundreds if not thousands of years we observe that people have established the divine through sound, from ritual drumming to the sonic surroundings encountered in large cathedrals. From Lüneburg's previous account one could argue that she used similar means to establish epistemic grounds in I Am a Priest.

It is assumed that the divine cannot be seen directly so it must otherwise be established, for example through the sense of hearing. The sense of smell is important too, as one might know from walking into a Catholic church and being exposed to the scent of incense. So, although the divine or the spiritual is something that we believe to go 'beyond' matters of the body, it is the body that hears and sees and smells it (or that which we 'know' to be hints and traces of it). If we hear the echo-drenched sound of certain organ chords and if we were brought up in a specific cultural environment, we know immediately that it is certainly sacred music we are hearing. In this context, we might 'sense' holiness and spirituality instead of 'mere' craftsmanship or compositional competence.

Lüneburg achieves plausibility through collaging several elements into one musical composition: the interview snippets (as rearranged by herself), her own violin playing, sound design, artistic ways of expressions deriving from classical contemporary music as well as the confirmation of historical and artistic narratives through encyclopaedia entries. This reminds us of what - according to Sarah Pink - the methodological approach of sensory ethnography offers when it strives "to bring researchers and their audiences close to other people's multisensory experiences, knowing, practice, memories 
and imagination." (Pink 2009: 132). While social sciences appear interested in opening their methodological palette to the styles of representation offered by sensory ethnography, and may work with artistic or semi-artistic elements, Pink stresses that "[w]ritten scholarship facilitates ethnographers' engagements in theoretical debate.” (ibid.: 135)

\section{Establishing the Context: An Artistic Research Perspective (Lüneburg)}

Following the strata of I Am a Priest and its connection to the project, it is worth noting that religion and spirituality were recurring topics on what-ifblog.net. Assuming that spirituality, as a part of one's identity, affords personal meaning, we explored rites and philosophical questions in a series of disparate blog posts that reached from the goddess Athena of the ancient Greek (Athena - Why I Like the Ancient Goddess), the power of transformation embodied in 'masks' (Masks for a New Identity), to Māori (Te Reinga...the Leaping Place of Spirits) and other rites, such as for example the guest blog Lines of Connection and Identity by Malika Sqalli, or Rose Petals, Queer Trauma, and Rituals - AA Bronson's Sacre du Printemps by myself. We explored in posts the connection between life and death (Catrinas / Submissions and Thoughts, and the artwork The Gods Envy Us) and of course between Santería and Catholicism (The Orichas of Santería). Being aware that our community came from a huge bandwidth of countries and cultural and societal systems, the methodology was explorative, including aspects of different cultures and religions. We investigated whether there are questions and inspirations shaped by spiritual and religious experiences that are valid for our community and that would include members from different cultural backgrounds.

What is the link between these disparate blog posts on spiritual experiences and I Am a Priest? While there is no causal succession to be found, we could talk about a continuity and overall significance to the project. None of the blog posts on this topic spoke about rules and directives in any of the world religions. Instead they dealt with questions of our own identity. They explored for instance how goddesses and gods from different cultures inspire the way we live our lives.

"Athena - why I like the ancient goddess

Athena.

Goddess of wisdom, courage, inspiration, civilisation, warfare and justice, strength and strategy, mathematics and the arts, crafts, and skill. ... She'll be an inspiration for me during the next month when I will try to get used to the art of blogging."

(Lüneburg, Athena - Why I Like the Ancient Goddess 2014) 
They asked how ancient cultural landscapes shape our identity; for example Anahit Mughnetsyan from Armenia in her guest blog, when she tried to grasp her connection to Armenia (quoted in full in chapter 8 'The Community's Voice'):

“The place where I live, Armenia, is 'holy' or 'Noah's,' ‘stone crosses,' 'paradise'.....land - they say.

Holy? Land?...

What if the place we live in is really holy, unique, special...?

Why I love living in Armenia. Really don't know. No one knows why he's born, rises, lives, loves in the place which we call homeland...

Identity? Roots? Again questions.

Yes and it's a pleasure to find your roots, and gain answers by walking on the holy+home+land..."

(Mughnetsyan, "Armenia-Homeland" by Anahit Mughnetsyan 2014)

We, the social media team of TransCoding, and our guest bloggers talked about rites and beliefs as part of the identity of a people, as for instance in my blog post on the place Cape Reinga, which is according to Māori lore the leaping place of spirits:

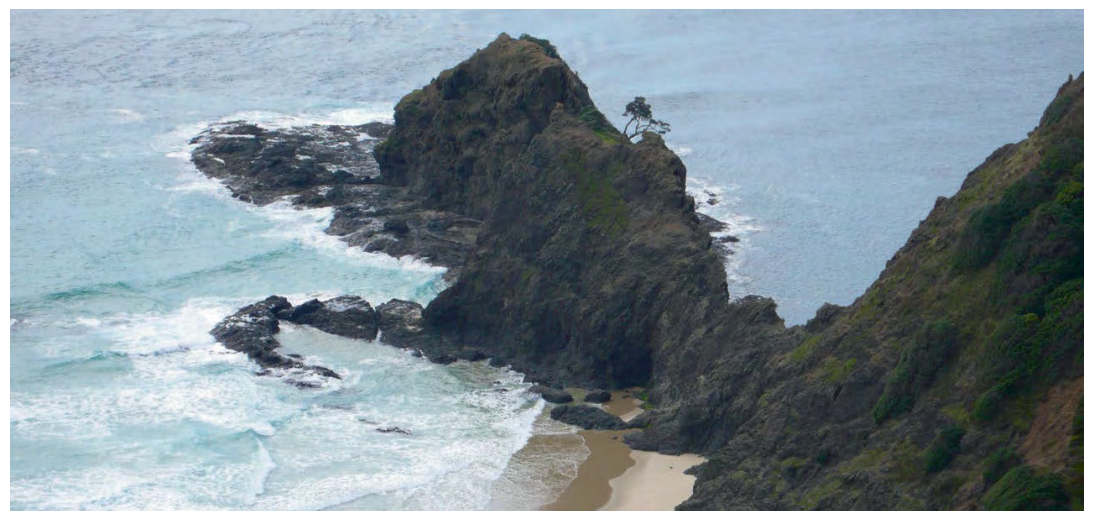

Fig. 3.1: Cape Te Reinga. Photo used by kind permission of Marko Ciciliani

“Te Reinga...the Leaping Place of Spirits

Cape Reinga is the northernmost point of New Zealand, on the narrow peninsula Aupori.

Here, the Tasman sea and the Pacific Ocean meet.

Here, according to ancient Māori lore the spirits of the deceased leap into the sea.

What a haunting, beautiful, spiritual place. 
'An ancient pohutukawa tree and a lonely lighthouse mark this special place. It is here that after death, all Māori spirits travel up the coast and over the wind-swept vista to the pohutukawa tree on the headland of Te Rerenga Wairua. They descend into the underworld (reinga) by sliding down a root into the sea below. The spirits then travel underwater to the Three Kings Islands where they climb out onto Ohaua, the highest point of the islands.' After a last farewell look at the land of the living, they descend again to the depths and continue their journey to the other world, the land of their ancestors, Hawaiiki-A-Nui.

Te Reinga 'the leaping place of spirits.'

We met Huinga, a Māori, who told us many stories about this landscape and about its people. He and I stood on top of Cape Reinga looking across the vast sea, when he sang this song for me.

A)) Song by Huinga at http://transcoding.info/english/book.html

- PART III CHAPTER 11 (3); text quote taken from http://www.doc.govt.nz/conservation/ historic/by-region/northland/ kaitaia/cape-reinga/."

In her guest blog post Lines of Connection and Identity Malika Sqalli continues the topical strand on Māori culture when she speaks about tattoo traditions in New Zealand among the Māori, and in Morocco within the Berber culture. The Māori Tā Moko tattoo tradition is understood as a sign of cultural, as well as tribal and personal identity with transcendental association. If the person that gets the Tā Moko wishes for it, it even links this person with their ancestors.

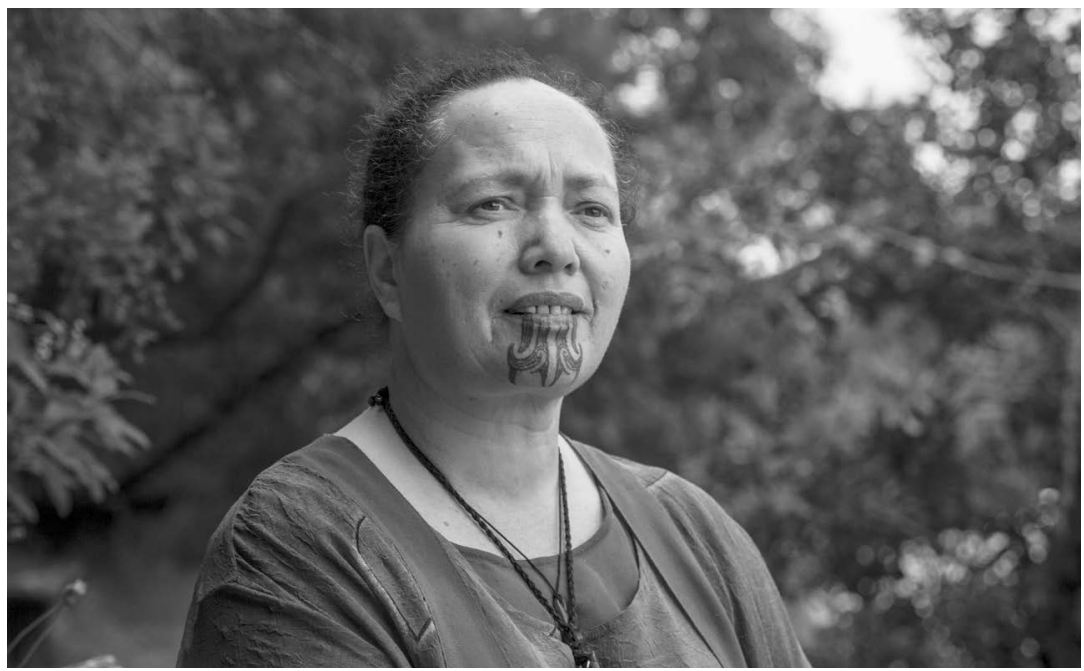

Fig. 3.2: Mere Taylor Tuiloma decided to take the step of getting her Moko done, a year after her husband's death. Photo used by kind permission of Malika Sqalli. 
Inspired by the post of a follower on Facebook, I asked how thinking about death and the finiteness of life shapes our everyday being. I transformed this into an artwork: The Gods Envy Us - part of the overall artwork of TransCoding:

Link to the video The Gods Envy Us:

http://transcoding.info/english/book.html - PART II CHAPTER 6 (1)

The visuals I used were images we had been given by our community in response to the Call for Entries / Catrinas and Creativity is Contagious.

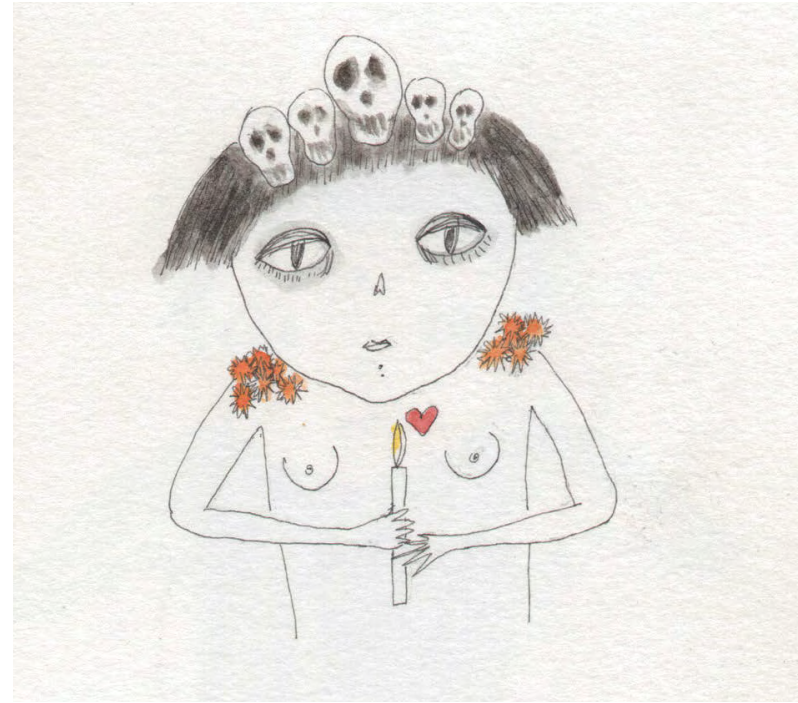

Fig. 3.3: Drawing of a 'Catrina' by Daniella Michelle in response to our call for Catrinas on the occasion of the Mexican Día de los muertos (Day of the Dead). The image is part of the visuals for The Gods Envy Us.

Those disparate blog posts and thoughts on spirituality, rites, life and death and identity formed one stratum through which I Am a Priest could come into being. They emerged as a field of questions on spiritual experiences and how those could be felt as part of our identity.

In I Am a Priest these strings came together and in it we find many elements our community members thought about in their own words, music or images: Aguirre introduces us to a number of his gods and goddesses, their tasks and their connections to Catholic saints, and he explains what they symbolise for the people who worship them. Through his words we hear about rites and 
ceremonies, the influence of different cultures on Santería (and his home country Cuba) and his personal priesthood. The motive of death appears in images that depict the ritual sacrifice of animals. In the soundtrack the audience can hear how religious feelings and ecstasy are established through the passionate and repetitive playing of the violin, the screams of the masses, and the ritualistic beating of a drum. Moreover, we experience Aguirre's personal way of worshipping - namely through classical music, part of his upbringing and his profession - in the sounds and the part of the violin, the instrument he studied. The material of the artwork (interview snippets, violin part and the soundtrack with its mixed elements from documentary recordings, electronic sounds and violin additions, and the images chosen for the video) offers the listener a reference to the immateriality and spiritual experience of the religion Santería.

In my opinion, the artwork has epistemological potential. It forms the node in the network of posts that refer to spiritual knowledge from different cultures, distant countries and even long-gone times. Through it I made notions of identity and spirituality available and possibly meaningful for an audience that in itself consists of many different cultural and personal identities.

\section{Establishing the Context: A Sociological Perspective (Kai Ginkel)}

From a sociological perspective it would be pointless to debate or even doubt any participant's personal associations regarding immateriality and spiritual experience. However, immateriality is not the focus of praxeological research. On the contrary, in praxeology and in other sociological disciplines scholars focus on what is material. Material is open to more or less direct observation, whereas any knowledge about the immaterial is treated as speculative. Ethnographic investigations can, however, focus on how the immaterial is talked about, written about and otherwise enacted in the form of clearly observable practice(s).

Ethnographic research of religious practice faces considerable challenges. As Murchison and Coats point out: "Attempts to understand contemporary religious practice, and its associated communities and identities, must take into consideration the way that these phenomena exist in both virtual and physical spaces, as well as the way that, in some instances, religion bridges or erases this dichotomy." (Murchison and Coats 2015: 988) This seems applicable to the way accounts of religious experience or spiritual identity are collected for TransCoding. Lüneburg addresses the question of what the link might be between disparate blog posts on spiritual experiences on the one hand and I Am a Priest on the other. Through these personal posts, spirituality and spiritual diversity 
are 'enacted' in a virtual space, namely the blog, by discursive action, i.e. the action of writing and reflecting on spiritual ideas. In the blog, those discursive artefacts of spiritual experience were linked via the concept of identity. In the Foucauldian sense of a "node within a network," the individual experiences and identities still form references and construct continuities that flow into the artwork I Am a Priest, but also connect community members across an abstract, yet personally interpreted concept.

Whereas Lüneburg contemplates how goddesses and gods from different cultures inspire our being, we could also change the perspective to the question of how gods and goddesses might be understood as reflections of societal living. In this sense, Anahit Mughnetsyan's spiritual blog post might be interpreted as a reflection of a strong notion of 'home' that is shaped by society and her cultural heritage, and an understanding of being 'rooted' in a particular place. How all of these aspects are enacted in everyday practice and also linked to questions of spirituality both in virtual and physical spaces might hypothetically serve as the subject of an ethnographic investigation.

In this way, the research results would take on the form of a text which, as Alvesson expresses it, is seen as the central part of the research project. "It tells a story, it uses a particular style, and includes much more than simply the reporting of data and a description of objective reality." (Alvesson, 2002 cited by Alvesson 2009: 159). While I am a Priest is not in a strict sense an ethnographic study, it achieves something similar: the artwork touches upon the multi-sitedness of rites, on enacted spirituality in everyday living and on the merging of both. 


\section{A COMPARISON OF DISCOURSES}

In the previous chapters we offered a general definition of artistic research and sociological ethnography and investigated their particular knowledge claims. Based on the case study I Am a Priest we studied data collection strategies, methods of analysis and structures of fieldwork, and tried to trace the construction of meaning making and knowledge gain in both fields. Additionally, we were interested in the articulation and communication of research findings. In doing this we were not seeking any singular valid perspective. Instead, we were studying the production of knowledge with regard to this specific artwork, and investigating how each method has a bearing on the kind of 'truth' that is being sought and how it is accordingly depicted.

In what follows, we strip down artistic research and ethnographic sociology by enlisting the raw factors of data collection, methodological set-up, 'going native,' 'being native' or 'making the familiar strange,' we look at power relations and ethical considerations, and representation and dissemination. In short, we look at the bones of both disciplines in an attempt to reveal the similarities and differences and to evaluate whether they might possibly inform each other in terms of methodological and discursive enhancement.

\section{Data Collection}

Both artistic research and sociology often work exploratively in an effort to understand human knowledge and experience. In artistic research data may be collected by doing art (such as performing, exhibiting, artistic software coding, sculpturing, etc., followed by critical reflection), by personal embodiment (for instance through my performing Toque), scientific experimentation, by connecting seemingly distant analogies or by exploring discontinuities to create new contexts within and through the artwork. Additionally, data collection happens via participant observation (to a great part from an inner, native perspective), by analysing case studies of one's own practice and that of other artists, and by any kind of qualitative or quantitative research that fits the inquiry.

In ethnography, participant observation (paired with an often prolonged engagement in the field), field notes and in-depth interviews or group interviews are well tested data collection tools, as is the use of non-interactive data sources such as documents and other unobtrusive measures. Recently, digital media for capturing images, recording sounds or interacting with social worlds have taken on an increasingly important role in data collection. They have also gained importance in the active involvement of researchers and participants (thereby granting participants a stronger sense of agency), and in the contextualisation, interpretation and representation of data. Social researchers Caroline Lenette 
and Jennifer Boddy state that "Proponents of the increasingly popular visualbased methods argue that complex experiences cannot be fully conveyed through textual interpretations alone (Pink 2006, 2007; Radnofsky 1996)." (Lenette and Boddy 2013: 72)

However, even if we can find a common approach with regards to tools and methods of data collection, there are still differences between artistic research and modern ethnography. In ethnography (classical, visual or sensory ethnography) data are collected to directly learn something about a selected group of people (or multiple sites and cultures), i.e. the field. In artistic research investigation could also mean to investigate through and on an abstract, aesthetical topic (through which we can explore more indirectly the notion of being human). Ethnographic researchers gather data on something that already exists, while in artistic research we often first have to create the data (the artwork and the process of creating art) that we base our inquiry on. Ethnographic researchers investigate others, whereas for art researchers their own art and arts practice are central to their work.

If tested methodologies of ethnography are applied as a tool for meticulous documentation and "analysis that does not necessarily emphasize the personal meaning or strongly subjective aspects of the research/event/experience," this might lend artistic research additional means "to address 'subjectivity' and prior knowledge as a complex mix of resource and blinder." (Alvesson 2009: 160 and 166) For those who investigate from inside their field or even from inside their own creative process and need to carefully counterbalance personal or subjective aspects of their analysis and narrative, such tools can be very important. Ethnography, by contrast, could gain from the self-reflective experience and investigative practice artistic researchers have with the handling of those data thoughts, ideas, interpersonal relations and communications - that are usually inaccessible to others because they are held 'under closure', i.e., in sociological terms, behind boundaries or within constructed identities that protect them from prying eyes.

\section{Methodological Set-Up}

In artistic research, scholars often engage in interdisciplinary work, combining methodologies from various fields that have to be tailored for each research project. In addition to the "experimentation in practice, reflection on practice and interpretation of practice," (Borgdorff 2012: 23) methods, techniques and approaches can be drawn from the humanities, the social sciences or the natural sciences. There is no one clear-cut singular method to follow. Often the conceptualisation of the research process is a continuous flow, especially since the research object, the artwork, is usually created within, through and as a result of 
the research process, and the researcher might at the same time be the research subject. The process of creating art, the process of performing or exhibiting art, and the product itself is necessarily a main part of the investigation. Research results are immediately fed back into the field and into the process of doing art and of thinking about art, and into the actual resulting artwork.

The research consists of the actual artistic activity and of the systematic and self-reflective investigation of it; the essential component is the artistic expression of the findings, often but not necessarily paired with a verbal report. Fundamental academic conventions such as 'methodology,' 'verifiability,' and 'reporting' are met, however, since artistic research is concerned with the production of art which in itself claims to be unique and individual, and 'replicability' is not primarily a criterion. Nevertheless, research findings are transferable insofar as artistic research is concerned with the development of the arts and future artists can base their (individual) work on the prior findings of artistic researchers.

In artistic research, methodology and research design for each new project have to be uniquely conceptualised and may differ fundamentally from one project to the other; classical ethnography, by contrast, prefers to use methodologies that are established, confirmed and time-tested. Subdisciplines of ethnography, such as ethnographic action research or visual or sensory ethnography, have nevertheless broadened the range of methods and approaches, leading to a different perspective on participant involvement, collaboration and feedback into the field.

"Further, visual methods induce more critical, reflexive methodologies (Pink, 2006), as interpretations of emerging data from participants' perspectives provide a comprehensive and enriching exploration of the social worlds of both researcher and participants. They also promote more collaborative methodologies than used in the past, as such methods involve the active involvement of both researchers and participants rather than, for example, passive involvement of participants in research based on observation (Pink, 2006)." (Lenette and Boddy 2013: 72)

Looking at future mutual methodological enhancement, it might be rewarding to apply the praxeological view on social phenomena (established as a network of practices, experiences, narrations, rearrangements and historical accounts) to the investigation of interactions between artists and their audiences and to the question how artworks construct and establish knowledge and meaning. Ethnographic research on the other hand could profit from the flexibility and multiplicity of methods and perspective offered by artistic research, paired with the art's rich means of sensory expression and its visual, audio and bodily interpretation of research outcomes. 


\section{Going Native, Being Native}

Whereas in many sociological debates 'going native' - today less offensively termed 'over-rapport' - is mostly considered a methodological flaw, artistic researchers are necessarily always 'natives' in the field they study; the creation of art and the reflection on context, the process of creation and the work itself, all come from an inner perspective. In his book The Reflective Practitioner on the professions of engineering, architecture, management, psychotherapy and town planning, social scientist Donald A. Schön argues that "[d]oing and thinking are complementary. Doing extends thinking in the tests, moves, and probes of experimental action, and reflection feeds on doing and its results. Each feeds the other, and each sets boundaries for the other" (Schön 1983: 280). Through artistic research we gain a detailed inside perspective on exactly this process: on artistic procedures, work development and how this leads to consequences in the artwork. 'Outside' researchers would not have access to this information, but only to the information granted by the artist(s).

Ethnography, on the other hand, sees an advantage in keeping objectivity and distance, to balance empathy and reflexivity and to avoid what Karen O'Reilly in her book Key Concepts of Ethnography calls the 'lure of acceptance.' However, she also observes that

\footnotetext{
"[n]ow that ethnography is likely to be undertaken in societies and communities where the ethnographer is already to some extent an insider (insider ethnographies), the problem of 'going native' is discussed less frequently. Furthermore, it is increasingly recognised that complete physical and emotional distance is neither possible nor even desirable." (O'Reilly 2009: 88 [emphasis in original])
}

When artistic research engages in enquiry from the inside, there can be disadvantages in the potential professional dependencies or obligations and the ethical sensitivities involved if a researcher is investigating while being part of the field he or she researches. The artistic researcher is vulnerable with regard to his or her professional position in the field which could theoretically even lead to self-censorship. There is a sense of personal exposure and the danger of exposing collaborating artists or colleagues in the field. The ethical guidelines of the Arts and Humanities Council include the 'confidentiality of information provided by research subjects, and anonymity of respondents.' (University of Glasgow, College of Arts - Research Ethics Policy 2017) However, it is not always possible to anonymise research subjects when describing arts practices or art works, and this may lead to the omission of controversial information. Also matters of professional self-branding might get in the way of honest reflection on both successes and failures, on opportunities and obstacles, and make open and critical self-reflectivity more challenging. 
In our opinion, it would be worthwhile for artistic researchers to look into and learn from the handling of closeness and professional distance in ethnography, and the problems that come with ethical considerations and self-reflectivity, two areas that are much more openly addressed in ethnography. Ethnographic researchers in turn could gain interesting insights from overcoming the closure of the field. Nevertheless, we see the need to carefully balance out the position of the professional and perhaps more 'objective' observer with the position of the inside expert who might be more intimately but also more 'subjectively' informed. In our opinion it is particularly important to find a balance here, especially with regard to shared authority over questions investigated and possibly controversial answers found. This problem links directly to the following section on power relations and ethical considerations.

\section{Power Relations and Ethical Considerations}

In The Order of Discourse, Foucault talks about "procedures of exclusion" linked with desire and with power. What does this mean with regard to the articulation and communication of research findings? Who has a claim to knowledge in the disciplines we look at? The actual members of the field researched or the researcher investigating the field? Who profits, and how? Who is allowed to say what? What does the interaction and relation between researcher and researched subject look like?

"In a society like ours, the procedures of exclusion are well known ... We know quite well that we do not have the right to say everything, that we cannot speak of just anything in any circumstances whatever, and that not everyone has the right to speak of anything whatever. In the taboo on the object of speech, and the ritual of the circumstances of speech, and the privileged or exclusive right of the speaking subject, we have the play of three types of prohibition which intersect, reinforce or compensate for each other, forming a constant grid which changes constantly ... It does not matter that discourse appears to be of little account, because the prohibitions that surround it very soon reveal its link with desire and with power." (Foucault, The Order of Discourse 1981: 52)

As we have seen, in ethnography, researchers aim for an analytical distance from their research subjects, even if they immerse themselves in a field via long-term engagement. Their understanding is based on a holistic approach, observing, perceiving and gathering facts to describe, reflect on and interpret place, people and activities. This does not come without drawbacks. A critical moment for example in ethnographical research can be the moment of leaving the field: 
"The ethnographer who courted others, who had seemingly limitless time to listen, is now revealed as a person who can no longer be bothered and is in a hurry to be off' (Rock: 36 ). To make matters worse, the ethnographer is off to expose what has been learned to the whole world. No wonder people can feel used." (Gray 2017: 463)

Ethnographers usually do not immediately feed their findings back into in the field or test their research results while still investigating. The research is done through studying and observing from an observational and analytical position, not through the process of active doing and reflecting as in artistic research. Also, ethnographic sociology does not necessarily aim for a transformation of the field or the subjects it studies. Furthermore, critical ethnographers address possible bias and the reinforcement of dominant power relations. They argue that the relationship between researcher and researched should not be hierarchical or exploitative, but based on consent and collaboration.

Artistic research is concurrently done for the researcher her- or himself, for the development of the arts and artistic practices, and for the (general and academic) audience. At best, artistic researchers simultaneously advance the domain and produce artworks that are shared with the public. Hence, the power lies with the artist-researcher and is shared with the onlooker. However, there are potential conflicts of interest that arise from the interplay of an interlocked professional research and arts practice.

The discipline of artistic research does not exist in a societal and professional vacuum; artistic researchers explore their art, and their investigation may be influenced by professional (financial, artistic and aesthetic) pressure from their peer group. Further, artistic researchers have to consider ethical pitfalls, since it can be difficult, sometimes almost impossible, to make research data from the arts domain and from colleagues anonymous.

Another power question that evolves around the relatively young field of artistic research is the question of legitimacy, distribution of money and reputation. A sociologist might ask whether artistic researchers count more than 'ordinary' artists, since they receive societal appreciation in the form of research money or academic titles. The arts world might ask whether artistic researchers perhaps represent an 'academisation' of art that has nothing to do with the 'real world' and challenges of arts practice. A traditional academic scholar might doubt the validity of art as a research outcome. In turn, an artistic researcher might face the special challenge of how to handle the tension between artistic practices within a relatively autonomous, dynamic art world and the standards and slower-paced reflective culture of academic research, which they share research money with. In our view, these questions deserve more future research from both the inner perspective of artistic and the more distant view of sociological ethnography. 


\section{Representation and Dissemination}

In artistic research the 'native,' the artist him or herself, claims to unearth and shape the knowledge embodied in the artwork, in the practice of art and in the reflection on it. The artworks themselves and their presentation constitute an indispensable component of the research outcome. Foucault describes the "living openness of history," that in its historical analysis can include the "use of discontinuity, the definition of levels and limits, the description of specific series, the uncovering of the whole interplay of differences" (Foucault, Archaeology of Knowledge 1972: 13); likewise, in artistic research, we investigate the "living openness of art.' Artistic researchers look at series, ruptures, transformations and thresholds, they are concerned with ideas (and sciences), and embrace and explore discontinuities. Meaning making and knowledge gain is ascertained through the artwork itself or through reflection on the process of doing art. Researchers not only witness to their own field and of their own art through their artistic work, they also touch on questions of humanity.

Artistic research has several audiences: the academic world, peers in the art world, and the general public who experience the artworks. The search for 'truth' and 'knowledge' through art has to allow for the fact that onlookers might understand artworks differently from the creator and draw their own individual conclusions. There is no claim to absolute truth, art is living and fragile insofar as it may be interpreted to have multiple meanings. By nature, its knowledge is contingent and invites ambiguity in the interpretation by the onlooker.

In Borgdorff's The Conflict of the Faculties, he postulates that artistic researchers should "elucidate both the process and the outcome [of their artistic research] in accordance with customary standards" and "reflect on the research process, and documentation of it in discursive form." (Borgdorff 2012: 25) However, for some artistic researchers turning their research findings into a verbal report for the purpose of meeting traditional academic standards is contrary to the idea and essence of artistic research. Far from being a mere illustration that goes along with the 'actual' research, as might be the case in sensory ethnography, to them the artwork is the main if not the only and indispensable outcome of their investigation. Borgdorff reports:

"It was warned ... with recourse to Heidegger, Foucault, Derrida, Deleuze, and Levinas, that true artistic research should resist, as an independent form of knowledge production, any kind of academisation. Through its focus on the singular, the aesthetic-affective, the transgressive, the unforeseen, artistic research should exemplify an alternative culture of knowledge." (Borgdorff 2012: 5)

Other scholars appreciate precisely this, the additional verbalisation of their artistic research; they see it as a tool to systemise and deepen their artistic 
investigation and practice, and as a matter of self-reflectivity and scientific rigour. A third party coming from traditional academia argues that the artistic production of knowledge has "potentially more in common with speculative philosophy and the knowledge criticism it practises than with scientific knowledge production." (Borgdorff 2012: 5)

The written research report is the traditional outcome in ethnographic research and has a special meaning for it. In the opinion of ethnographer John van Maanen, the written report represents the culture of a field that needs to be interpreted by the fieldworker. He clearly distinguishes between natives' points of view and the interpretation of culture that the ethnographic researcher develops. It's not that the culture makes itself known through words, actions and artefacts of its members; rather, the independent interpretation by the researcher constitutes the representation of the culture and thereby the (valid) research outcome in ethnography.

"A culture is expressed (or constituted) only by the actions and words of its members and must be interpreted by, not given to, a fieldworker. To portray culture requires the fieldworker to hear, to see, and, most important for our purposes, to write of what was presumably witnessed and understood during a stay in the field. Culture is not itself visible, but is made visible only through its representation ... The fieldworker must display culture in a narrative, a written report of the fieldwork experience in self-consciously selected words. Ethnography is the result of fieldwork, but it is the written report that must represent the culture, not the fieldwork itself. Ethnography as a written product, then, has a degree of independence (how culture is portrayed) from the fieldwork on which it is based (how culture is known)." (Van Maanen 1988/2011: 3 f.)

In this understanding, the meaning of culture is not accessible through 'being an insider' but through an academic perspective established in specific methods of data collection, analysis, reflection and, finally and necessarily, academic writing. The Sensory Ethnography Lab (SEL), an experimental laboratory at Harvard University, acts in contrast to this assessment. They explore - as they call it - innovative combinations of aesthetics and ethnography and use analogue and digital media to explore the aesthetics and ontology of the natural and unnatural world. In their own words they provide

"an academic and institutional context for the development of creative work and research that is itself constitutively visual or acoustic - conducted through audiovisual media rather than purely verbal sign systems - and which may thus complement the human sciences' and humanities' almost exclusive reliance on the written word and quantification. It opposes the traditions of art that are not deeply infused with the real, those of documentary that are derived from broadcast journalism, and those of visual anthropology that mimic the discursive inclinations of their mother discipline." (Castaing-Taylor et al. 2010) 
They seem, however, to be the exception. Although with the development of sensory ethnography the palette of methodological tools has been expanded, even expert Sarah Pink supports the claim of academic writing as being the most important tool for the communication of research findings:

\footnotetext{
"The written word is the most embedded and developed form of ethnographic representation, and a sophisticated technique for scholarly communication. It remains the dominant method of relating the findings, methodologies and theoretical implications of ethnographic studies generally, as well as those that attend to the senses." (Pink 2009/2015: 132)
}

Whereas artistic researchers still debate among themselves which form of representation may be valid for displaying the outcome of their research, and some struggle with the connotations of the written report, ethnographic sociologists only slowly and cautiously open their communication and research representation to sensory and artistic expression. The gap is still wide but holds the potential of added value for both sides.

\section{Quality Assessment and Development of Our Disciplines}

Two questions remain: how do we establish quality standards and how do we further the (methodological) development of our respective disciplines?

Artistic researchers need to continuously discuss and develop skills and methods that enable them to adopt an inquiring approach to their own art, to observe the process of doing art with analytical awareness and a broad choice of methodological tools, and to assess the effect they have on their domain and - through their art - on their audience. Embedding their research in an interdisciplinary approach or an interdisciplinary team can provide a point of reference and accountability, and support their pursuit of adequate quality criteria and standards of judgement. The typical first-person perspective of artistic research could for instance be supported by second-person and thirdperson research; alternatively, the use of multi-disciplinary methodologies could help to counterbalance subjectivity and reduce the defensiveness that might result from research 'at home,' within one's own professional field. However, what organisational behaviour scholar Judi Marshall and action researcher Geoff Mead say about first-person action research can equally be true for artistic research in the most positive sense:

\footnotetext{
"Without wishing to render oneself or others unnecessarily vulnerable, it may be that this 'edginess' is a possible marker of quality in first-person action research, an indication of a willingness to work at one's 'learning edge.” (Marshall and Mead 2005: 237)
} 
For both ethnography and artistic research, we would like to pose the question of how 'being native' as a legitimate access to knowledge and information can further be systemised and rendered useful. We argue that inquiry in each of these domains can gain value and depth not only by interaction and selfreflection by someone who is both 'native' and observing researcher, but also by the inclusion and participation of the field while having a critical, systematic and analytical research approach. The investigation of 'context,' the construction of 'realities' and the evaluation of meaning in each of these fields could be enriched by the juxtaposition of theories, ideas and the practice of observation from a certain distance used in ethnographic research, and the explorative practices and inquiry from the inside used in artistic research.

For this to be possible we need to carefully assess and differentiate autonomies and dependences in our domain and the field we investigate, and change our core questions from 'What has really happened?' or 'What is really said?' to 'Which methodological tools render the most interesting result in our search for meaning?', 'What do we learn from the different perspectives available?', and 'To what extent does this method drive our analysis and understanding of questions of humanity forward?'

Following Foucault, we propose to strive for a 'total description' that draws all phenomena around a single centre - a principle, a meaning, a spirit, a worldview, an overall shape.” (Foucault, Archaeology of Knowledge 1972: 10) 


\section{CONCLUSION}

With TransCoding we came from the field of contemporary art music and multimedia art, a field that is often considered 'highbrow,' intellectual, and unapproachable, a field that produces art for a minority and experts. And yet we succeeded in establishing an online community of mainly non-expert, creatively interested people that picked up on our topic of contemporary art and was actively involved in the making of new artworks. In the course of the project we developed a successful model of interaction and involvement (as discussed in Part II of this monograph).

The work with the community led to a whole body of artworks that were generated from different premises: firstly, those that were built on our calls for entry, for which the community partly used material TransCoding provided, adding their own resources; secondly, works that were based on inspiration from the community but otherwise produced non-collaboratively either by single community members or by myself; thirdly, the artworks Read me and Slices of Life that combined the creative potential of the community with my own artistic ideas; and finally, the accumulation of creative works and thoughts on all of TransCoding's social media channels, which I consider a separate participatory artwork in itself.

We as a team, and especially I as lead artist and communicator, experienced not only successful long-term interaction, but also ruptures and discontinuities within the process that can be traced well into the artworks and into the development of the content and design of the social media channels. Therefore, I would like to conclude this text by discussing the viability of this model of participatory art for future arts practice.

\section{TransCoding - A Model for Future Arts Practice?}

What practical and theoretical problems emerge from a review of this project? The decision to work and produce under the premises of participatory art and via social media was fed by my desire as a performer to reach people beyond the anonymous relationship between the sole creative artist and the 'consuming 
audience' and to exchange with them. With the project I strove to share and democratise the creative process, and to define the relationship between artist and audience/community as one of permeability, mutual influence and empowerment. Whether this led to a fruitful interactive exchange between contributors and artists, adding meaning to both groups, was energetically discussed.

\section{Participation of the Community}

The original group of artists was not comfortable with the community as a partaking agent in the creative process. In their perception the communication and interaction with the community did not add value to their artistic practice. On the contrary, they felt hindered in their creative process, they found it difficult to integrate the contributions from non-professionals, and they feared for the quality of their work and their reputation in the arts scene. In consequence, the original group of artists left the project after the first year. I had to accept that what seemed to be stimulating for the community was not necessarily perceived as fruitful for the personal artistic practice of my creative collaborators, and that each individual artist had to balance the pressure they felt from the professional field they worked in and to draw consequences from that. However, the community itself conveyed interest, pride and joy in being involved in TransCoding, and I felt inspired and supported through them. The interaction and joint participative creative work changed my self-concept as artist and my way of practising art.

\section{Social Media}

Social media are characterised by a fleeting momentum. They need to be constantly nurtured to ensure attention from the public. We had to regularly and frequently feed the main blog what-ifblog.net with content and subsequently spread it across our social media channels. This required research on the most suitable topics to present in order to balance our field of interest with that of our community and to frame our posts in an appealing form that would pique the curiosity of our community members. Additionally we had to develop calls for entries, encourage people to participate in our challenges and quickly follow up comments, questions or submissions; all of this in addition to the research and development of the artwork. The enormous workload makes this model of participatory art via social media less viable for an artist who lacks significant financial backup and further human resources, especially in the field of contemporary art music, where money resources are scarce and honoraria small. TransCoding was only possible because the research and art production was based on substantial research funding from the Austrian Science Fund. 


\section{Crossover Culture}

Since we were funded through the PEEK-programme of the Austrian Science Fund, the 'Programme for Arts-Based Research,' I was financially independent of the art market and could - with minimised risk - try new aesthetic and conceptual paths in the development of TransCoding's artwork.

What are the influences of popular art in the overall project? I wrote story elements and narratives into both the artworks, Read me and Slices of Life, that emerged from the interaction with and the contributions from the community. Additionally, I used the human voice (without processing or sound manipulation) to directly convey the story. Both stand in contrast to the intellectual abstraction often favoured in Western contemporary art music.

I use illustrative elements of sound design throughout all segments of the composition Slices of Life to support the narratives, while consciously treating sonic phenomena as "complex entities with various layers and connotations, which [again] contrasts with a more abstract parametric approach to sound, that was dominant after World War II." (Ciciliani 2017: 33) However, I also often use sounds that are explicitly drawn from the sonic world of contemporary art music, such as extended violin techniques and microtonal harmonics.

The instrumentation of Slices of Life encompasses elements of classical, popular, folk and contemporary art music and crosses boundaries in many directions. I include an instrument that is a core instrument of Western classical culture, the acoustic violin. At the same time, I employ instruments from popular music such as analogue synthesisers and electric guitars to create the sonic world of Slices of Life; sounds of a broken accordion, inside-piano sounds and ringing bells with microtonal beatings could be considered representative of contemporary art music. The instruments merge with the voices of international community members who recorded the narration for me and with my own voice.

Remix was an issue not only in the calls for entry on the blog but also in the artworks themselves, Slices of Life and Read me. I worked with fragments of an Armenian classical contemporary song provided by composer and community member Anahit Mughnetsyan and her friend, the excellent soprano Heghine Ohanyan, and juxtaposed this with a Korean folksong beautifully chanted by community member Gloria Guns. With their permission I remixed their compositions and combined them with quotations from Johann Sebastian Bach, Afghan folk music and elements from pop music. I merged styles from classical romantic music with pop rhythms and electronic processing.

Additionally, the form of Slices of Life was inspired by pop albums. It consists of a series of short musical stories between two and five minutes long that together form the overall narrative. Soundscapes, documentary-style recordings, personal interviews, images that captured everyday life, and of course the texts of our community members were woven into the mix thus removing contemporary (classical) music and media art from their intellectual and secluded spheres into 
realms that are associated with our lives, dreams and fantasies, our otherness and our belongings.

The primary question in the foreground of my artistic work was whether I would be able to further cross over between high art and popular art by offering creative and intellectual incentives, while on the other hand receiving, carefully listening to and channelling the community's own creative voices. Nevertheless, the intention was that the artistic outcome could stand its ground within the field of contemporary art music.

\section{Artistic Research and TransCoding}

Why was the discipline of artistic research the right approach to set up, shape, conduct and investigate this particular project? I would like to offer four reasons.

Knowledge gain and communication through the arts defines the discipline of artistic research at its core.

The entire model of TransCoding was constructed around communication through art. However, we enlarged the communicative spectrum from 'the artwork that speaks to its audience' to a direct exchange between the professional artist and the community. We communicated via chats, blog posts, comments, likes and joint artistic enterprises, and last but not least we also communicated and learned through the artworks created by our members, by me, and jointly by them and me.

Artistic research produces new insights from an inside perspective.

Artistic research in general and this project in particular enable me to investigate the nature of art and how to create meaning and gain knowledge through the arts. Through the challenges of artistic research in general, through the systematic analysis and reflection of my artistic activity and in particular through a research enterprise such as TransCoding, I have grown as an artist, expanded my boundaries and re-invented my role as performer: from a sole interpreter and 'servant' of classical music, I have developed into a communicator with my audience, and last but not least into a creator with my own compositional practice. My perspective has widened: from being somebody who 'just' reflects on the work of a classical performer, I have become a researcher who employs her own artistic work as the basis for knowledge gain.

Artistic research enriches its domain and feeds back into the field it investigates.

TransCoding was undertaken with the direct involvement of a community that influenced the resulting artworks. I was part of the field I investigated and fed insights directly back into the community. Community members had the 
opportunity to gain a level of individual empowerment through the production of art in the framework of TransCoding. My interaction with them, on the other hand, changed my perspective on my own domain, contemporary art music. We expressed this process in the participatory and crossover artworks Slices of Life, Read me and the total of the participatory artwork of TransCoding. In doing so, we questioned the conception and (widely accepted) nature of an artist's exclusive authority in art production. The outcome of the process benefitted not only the (arts) domain but also of the people involved.

Artistic research is based on the notion that knowledge is inherent in aesthetic experience and is produced through the process of making and presenting art.

On the basis of the aesthetic and sensory dimension of artworks, insights can be experienced by creator, onlooker and listener alike and are open to a range of interpretations that can be turned into personal experience for individual users. In the case of TransCoding, that meant that through participating in the project, and through their general involvement and creative engagement with our common topic 'identity,' our members could (at least theoretically) gain deeper knowledge and extract new understandings of (participatory) contemporary art and of 'identity' from our own contributions, the submissions of others and the information the blog offered. Through the artworks, the act of performing, the participation, the online documentation and, last but not least, the live performances of the work, our audience, the online community and I myself could explore different forms of 'identity' with mind, body and soul. Thus, each of us could assess knowledge on multiple levels, something we wouldn't have achieved by reading an academic text alone.

Working in a mixed team combining multiple methods of (interpretive) research helped us to investigate from various perspectives - as observers from outside and participants from the inside - and approach knowledge from several angles. We claim to thus have come to a richer and more detailed picture in our findings, while having submitted our analysis and interpretation to a methodological reflexivity that was educated and trained in different research disciplines.

\section{Who Benefitted from TransCoding?}

We observed that our project fostered curiosity for our art, and that our community established a habit of watching, experimenting and co-creating within and beyond our project. Our numbers attest to this: there were over 20,300 views on the blog alone during 2014 and 2015; in the one week February 15-22, 2016 we engaged 141 people on Facebook; and with more than 1,200 followers on our various social media channels, we reached far more people 
than would have been possible had we simply stayed within the contemporary music scene. However, what individual community members explicitly gained - and this includes all stakeholders, our online community, audiences and the TransCoding team itself - and on what level it altered them, was up to them.

In conclusion, I would like to quote Malika Sqalli, from Rabat, Morocco, a traveller and photographer, who wrote to me on Facebook on February 23, 2016:

$\mathrm{Hi}-$

I had a look at the project and the link you sent me - I so love the collaboration of so many from creatives to scientists. ... sensing the space and time and questioning the lines that boundaries are. It made me think that your project is about ... words and description and boundaries of identity.

When we define something we draw a line around it through words which are ultimately lines when written and soundwaves when spoken. ...

I will get on your project - it will take me on a little wander in fairyland and come up with a new personal mythology! ...

Take care and I will be in touch soon.

$\mathrm{xx}$

(Malika Sqalli in a Facebook message to the author on February 23, 2016). 


\section{ACKNOWLEDGEMENTS}

Many people have supported me in the research project TransCoding and in the production of this text and I wish to thank them.

I am especially indebted to the team's social media manager Clio Montrey, who - being herself an artist - supported this project wholeheartedly and with unfailing enthusiasm. Her communication skills and aptness in the use of social media were immensely conducive to the success of the work with the TransCoding community.

My gratitude goes also to my team colleague Kai Ginkel who observed the project as sociologist and contributed with critical comments and reflections. His curiosity about artistic research led to the comparison between discourses in ethnographic sociology and artistic research. Both Clio and Kai gave me invaluable critical input while writing this text for which I am very grateful.

As representatives of our active contributors who carried the project with their beautiful stories, images, sounds and supportive, inspiring and critical comments, I would like to thank two of our most engaged followers:

Anahit Mughnetsyan, choir conductor and composer from Armenia, let me feel that this project was important to her and that she thought it was valuable for future generations. Her beautiful music Megha is woven into Slices of Life and both her guest blogs were influential in the development of the project. Thank you for your wonderful support, honest communication and your friendship.

Gloria Guns, human rights lawyer and front-woman of the band Scary Bear from Canada, submitted music, text and images to our calls for entry, wrote guest blogs, contributed with text, image and music to her own Read me installation and inspired me on various levels. I incorporated her remix Fan Death into Slices of Life and the finishing segment, Charter of Human Rights, is directly influenced by her. Thank you for your engagement, support and inspiration.

I am grateful to the Austrian Science Fund, which offers the innovative funding programme for arts-based research that afforded my team and myself the opportunity to undertake this creative research adventure. The University 
of Music and Performing Arts, Graz, which in the second year of the project took me into their ranks of researchers and gave the project a new institutional basis, provided a rich research and arts environment in which the project could blossom. I thank the university and my wonderful colleagues for support, personal encouragement and inspiration.

I would also like to thank Hannes Raffaseder who, as former head of St. Pölten University of Applied Science, prompted and encouraged me to submit this arts research project at the Austrian Science Fund (FWF), and who backed my application with his institution.

My gratitude goes to the publisher RUUKKU, Studies for Artistic Research, for giving me permission to use large excerpts of my paper From 'Highbrow Art' to Participatory Culture - A Potential for Change (2017) at different places in this text, and to Sarah Patey for her meticulous and analytical proofreading.

I would like to add many thanks to my partner Marko Ciciliani, composer, performer and artistic researcher himself, who supported me throughout all the ups and downs of the project with never-ending patience, warmth, humour, creative ideas, critical reflection and so much happiness and joy.

Finally, my greatest gratitude goes to the members of the TransCoding community. They were truly inspiring, encouraging and surprising, and so creative with word, sound and image. They came from Armenia, Austria, Bulgaria, Canada, Croatia, Cuba, England, Ireland, France, Germany, Great Britain, Mexico, The Netherlands, The Philippines, Poland, Russia, Romania, Spain, USA, New Zealand and many more countries. Some of them I had the opportunity to meet personally, others were anonymous followers at first and became friends. I very much hope I will eventually be able to bring the artworks to which they contributed so much to their home countries.

I cannot thank them enough. 


\section{BIBLIOGRAPHY}

\section{A}

Adler, Patricia A., and Peter Adler. 1987. Membership Roles in Field Research. Thousand Oaks: SAGE Publications. https://doi.org/10.4135/9781412984973

Aguirre, Louis. 2013. "Re: Solo." E-mail to Barbara Lüneburg on his violin solo Toque Oct. 6, 2013

—. and Barbara Lüneburg. 2016. "Lyrics of the artwork Slices of Life - I Am a Priest” by Barbara Lüneburg. Vienna.

Alvesson, Mats. 2009. "At-home ethnography: Struggling with closeness and closure." In Organizational Ethnography: Studying the Complexity of Everyday Life, Editor: Sierk Ybema, Dvora Yanow, Harry Wels and Frans Kamsteeg. London: SAGE Publications Ltd. https://doi.org/10.4135/9781446278925.n9

Austin, D. and Forinash, M. 2005. "Arts-based inquiry." In Music Therapy Research, Editor: B. Wheeler, 458-471. Gilsum: Barcelona Publishers.

B

Bishop, Claire. 2012. Artificial hells: participatory art and the politics of spectatorship. London: Verso.

Borgdorff, Henk. 2012. The Conflict of the Faculties - Perspectives on Artistic Research in Academia. Leiden University Press. Accessed on May 26, 2017. https://blogs.brighton.ac.uk/ttwl/files/2016/05/H-BorgdorffThe-Debate-on-Research-in-the-Arts-14d694g.pdf.

Bueger, Christian. 2014. "Pathways to practice: praxiography and international politics." European Political Science Review 6 (3): 383-406.

https://doi.org/10.1017/S1755773913000167

Butler, Judith, interviewed by Max Miller. 2011. Judith Butler: Your Behavior Creates Your Gender. https://www.youtube.com/watch?v=Bo702LYATDc. Jan. 13. Accessed on July 15, 2017.

C

Calleja, Gordon. 2011. In-Game From Immersion to Incorporation. Cambridge, Massachusetts: MIT Press. 
Carboni, Marius. 2011. "Changes in marketing in the classical music business over the last 20 years.” 2nd Vienna Music Business Research Days: Young Scholar's Workshop Innovations in Music Business.

Castaing-Taylor, Lucien, et al. 2010. Sensory Ethnography Lab: Harvard University. Harvard University. Accessed on June 19, 2017. https://sel.fas.harvard.edu/.

Ciciliani, Marko. 2017. Music in the Expanded Field - On Recent Approaches to Interdisciplinary Composition. vol. 24, in Darmstädter Beiträge zur Neuen Musik, Editor: Michael Rebhahn and Thomas Schäfer, 23-35. Mainz: Schott Verlag.

—., Gerhard Eckel, Barbara Lüneburg, David Pirrò and Hanns Holger Rutz. 2016. Programme note for the concert "Trans" at Minnoritenkloster Graz on Nov. 12, 2016.

Coffey, Amanda. 2004. Reconceptualizing Social Policy: Sociological Perspectives on Contemporary Social Policy. New York: Open University Press.

Cohn, Carol. 2006. "Motives and Methods: Using Multi-Cited Ethnography to Study National Security Discourses." In Feminist Methodologies for International Relations, Editor: Brooke Ackerly and Jacqui True, 91-107. Cambridge: Cambridge University Press. https://doi.org/10.1017/CBO9780511617690.007

Cornwall, Andrea, and John Gaventa. 2001. From users and choosers to makers and shapers: Repositioning participation in social policy. Working Paper 127, Brighton: Institute of Development Studies, IDS.

Cox, Arnie. 2011. "Embodying Music: Principles of the Mimetic Hypothesis." Music Theory Online (Theory, MTO - a journal for the Society of Music) $17(2)$.

D

Deci, Edward L., and Richard M. Ryan. 2000. "Intrinsic and Extrinsic Motivations: Classic Definitions and New Directions." Contemporary Educational Psychology, 54-67.

Dellwing, Michael, and Robert Prus. 2012. Einführung in die interaktionistische Ethnografie. Soziologie im Außendienst. Wiesbaden: VS Verlag für Sozialwissenschaften. https://doi.org/10.1007/978-3-531-94265-0

Drum, The. 2012. Case Study: How Gangnam Style went viral with a strategic marketing campaign from YG Entertainment / The Drum. The Drum. Oct. 30. Accessed on Oct. 6 2017. http://www.thedrum.com/ news/2012/10/30/case-study-how-gangnam-style-went-viral-campaignyg-entertainment.

E

Elliott, T.C., interviewed by Clio Montrey. 2015. \#WhatIfDrone-Remix (Sept. 9). 


\section{F}

Feathers, Laura. 2016. \#WhatIfSoundSamples / Laura Feathers. Feb. 6. Accessed on Sept. 8, 2017.

https://what-ifblog.net/2016/02/06/whatifsoundsamples-laura-feathers/. -., Anahit Mughnetsyan, Alina Murzakhanova, Ricardo Tovar Mateus and Sabina Ulubeanu. 2016. "Comments on Facebook Profile of Kati Mi." Facebook. May 3. Access on Sept. 16, 2017. https://www.facebook.com/profile.php?id=100006153074849.

Ficara, Ken, interviewed by Clio Montrey. 2015. \#WhatIfDrone-Remix (Sept. 2). Flüh, Torsten. 2016. Neue Musik von der Urbevölkerung und der Blog-Community - Barbara Lüneburg bei und das Abschlusskonzert mit dem DSO von Ultraschall 2016. Feb. 3. Accessed on Oct. 30, 2017. http:// nightoutatberlin.jaxblog.de/post/Neue-Musik-von-der-Urbevolkerungund-der-Blog-Community-Barbara-Luneburg-bei-und-dasAbschlusskonzert-mit-dem-DSO-von-Ultraschall-2016.aspx. Foucault, Michel. 1972. Archaeology of Knowledge. Translated from the French by A. M. Sheridan Smith. New York: Pantheon Books.

-. 1981. "The Order of Discourse." In Untying the text, Editor: Robert Young. Boston: Routledge \& Kegan Ltd.

Frogett, Lynn, Mervin Conroy, Julian Manley, and Alastair Roy. 2014. "Between Art and Social Science: Scenic Composition as a Methodological Device." Forum Qualitative Sozialforschung / Forum: Qualitative Social Research, 15 (3). Accessed on Oct. 31, 2017. http://www.qualitativeresearch.net/index.php/fqs/article/view/2143/3684\#g5.

G

Gauntlett, David. 2007. Creative Explorations - New approaches to identity and audiences. New York: Routledge.

Geertz, Clifford. 1973. The Interpretation of Cultures. New York: Basic Books. Gobo, Giampietro. 2008. Doing Ethnography. London: SAGE Publication. http://dx.doi.org/10.4135/9780857028976

-. and Andrea Molle. 2008/17. Doing Ethnography. 2nd. Sage.

Graham, Linda. 2013. "The Product of Text and 'Other' Statements: discourse analysis and the critical use of Foucault.” vol. 43, in The Power In / Of Language, Editor: David R. Cole and Linda Graham. Wiley-Blackwell.

Gray, David E. 2017. Doing Research in the Business World. London: SAGE Publications.

Green, Anthony R. 2016. \#GYHAFY Piargno. Editor: Barbara Lüneburg. March 25. Accessed on Sept. 7, 2017. https://what-ifblog.net/2016/03/25/gyhafy-piargno/\#respond.

-. 2016. Bio -- AnthonyR.Green. accessed on Sept. 7, 2017. https://www.anthonyrgreen.com/bio/. 
Guns, Gloria, interviewed by Clio Montrey. 2015. \#WhatIfDrone-Remix (Sept. 7).

-. 2015. Fan Death. Accessed on Aug. 312017.

https://soundcloud.com/gloriaguns/fan-death.

H

Hegner, Victoria. 2013. "Vom Feld verführt. Methodische Gratwanderungen in der Ethnografie." FQS Forum Qualitative Social Research. Forum Qualitative Sozialforschung 14 (3). Accessed on Jan. 12 2016. http:// www.qualitative-research.net/index.php/fqs/article/view/1957/3596.

—. 2004. "Wo Hexen hexen: Die neuheidnische Hexenreligion und Orte ihrer Praxis in Berlin." In Religiosität und Spiritualität: Fragen, Kompetenzen, Ergebnisse, Editor: Helmut Groschwitz and Anja Schöne. Münster: Waxmann Verlag.

Hughes, John A., Dave Randall, and Dan Shapiro. 1992. "From Ethnographic Record to System Design: Some Experiences from the Field.” Computer Supported Cooperative Work (Kluwer Academic Publishers) 1 (3): 123-141. https://doi.org/10.1007/BFo0752435

I

Ingold, Tim. 2014. "That's enough about ethnography!” HAU: Journal of Ethnographic Theory (Centre of Ethnographic Theory SOAS, University of London) 4 (1): 383-395.

Irele, F. Abiola, and Biodun Jeyifo. 2010. "Santería”. vol. 1, in The Oxford Encyclopedia of African Thought, 305. Oxford University Press.

Ivanovic, aka Lukatoyboy. 20I2. Blind Tape Quartets's Biography.

Nov. 29. Accessed on Oct. 12, 2017.

https://www.last.fm/music/Blind+Tape+Quartets/+wiki.

J

Jank, Sabine. 2012. „Strategien der Partizipation.“ In Das partizipative Museum, Editor: Susanne Gesser, Martin Handschin, Angela Janneli and Sibylle Lichtensteiger. Bielefeld: Transcript.

https://doi.org/10.14361/transcript.9783839417263

Jenkins, Henry (P. I.), Ravi Purushotma, Margaret Weigel, Katie Clinton, and

Alice J. Robison. 2009. "Confronting the Challenges of Participatory

Culture - Media Education for the 21st century." Massachusetts

London, England The MIT Press Cambridge. Accessed on Oct.

23, 2014. https://mitpress.mit.edu/sites/default/files/titles/free_ download/9780262513623_Confronting_the_Challenges.pdf.

Jenkins, Henry, Sam Ford, and Joshua Green. 2013. Spreadable media: Creating Value and Meaning in a Networked Culture. New York: New York University Press. 


\section{K}

Kaplan, Andreas M., and Michael Haenlein. 2011. "The early bird catches the news: Nine things you should know about micro-blogging." Business Horizons (Elsevier) 54: 105-113. https://doi.org/10.1016/j.bushor.2010.09.004

Kruse-Weber, Silke, and Barbara Lüneburg. 2016. E-mail exchange. Graz, Sept. 30.

$\mathbf{L}$

Lakoff, George, and Mark Johnson. 2003. Metaphors we live by. London: The University of Chicago Press.

https://doi.org/10.7208/chicago/9780226470993.001.0001

Leadbeater, Charles. 2008. We-Think: Mass innovation, not mass production. London: Profile Books.

Lenette, Caroline, and Jennifer Boddy. 2013. "Visual ethnography and refugee women: Nuanced understandings of lived experiences." Qualitative Research Journal (Emerald Group Publishing Limited) 13 (1): 72-89. https://doi.org/10.1108/14439881311314621

Lüneburg, Barbara. 2014. 5 Questions that are Important to Us. June 2. Accessed on Oct. 21 2017. https://what-ifblog.net/2014/04/18/you-and-us/.

- 2013. "A holistic view of the creative potential of performance practice in contemporary music." Brunel University Research Archive. January. Accessed on Nov. 14, 2017. http://bura.brunel.ac.uk/handle/2438/7512.

- . 2014. Athena - why I like the ancient goddess. March 1. Accessed on Nov. 16, 2017.

https://what-ifblog.net/2014/03/01/athena-why-i-like-the-ancient-god/.

-. 2015. Call for Entries - Drone Remix Contest. Barbara Lüneburg.

Aug. 17. Accessed on November 28, 2017. https://what-ifblog.net/2015/08/17/call-for-entries-drone-remix-contest/.

-. 2016. Call for Entries: \#GYHAFY - Give yourself a holiday away from yourself. Feb. 3. Accessed on Sept.7, 2017. https://what-ifblog.net/2016/02/03/ call-for-entries-gyhafy-give-yourself-a-holiday-away-from-yourself/.

-. 2014. City Hopping for a New Identity - the Sky is No Limit. April 24. Accessed on Oct. 18, 2017. https://what-ifblog.net/2014/04/24/city-hopping-for-a-new-identity/.

-. 2017. "From 'Highbrow Art' to Participatory Culture - A Potential for Change," RUUKKU Studies in Artistic Research (Research Catalogue (RC), RUUKKU portal) 6.

https://www.researchcatalogue.net/view/253119/253120

- 2014. Making of: Vienna Weekend Thoughts 1 - We Think Therefore We Are. Oct. 24. Accessed on Aug. 30, 2017. https://what-ifblog.net/2014/10/24/vienna-weekend-thoughts-1-wethink-therefore-we-are/. 
-. 2014. Te Reinga...the Leaping Place of Spirits. Nov. 17. Accessed on 16.

November 2017.

https://what-ifblog.net/2014/11/17/te-reinga-the-leaping-place-of-spirits/.

-. 2014. What-ifblog.net / About. Feb. 20. Accessed on Oct. 1, 2017.

https://what-ifblog.net/about/.

-. 2014. Who is Damian, Lia and Barbara...? A Remix Challenge on YouTube.

May, 15. Accessed on Oct. 21, 2017.

https://what-ifblog.net/2014/05/15/who-is_remix/.

-. 2014. Window shopping for a new identity. Vienna. Spring 2014. April, 4.

Accessed on Oct. 18, 2017.

https://what-ifblog.net/2014/04/15/who-are-you-this-spring/.

Lüneburg, Barbara, and Clio Montrey. 2015. SoundCloud Group / What-ifblog. net.

Editor: Barbara Lüneburg. Aug. 17. Accessed on Aug. 21, 2016.

http:// soundcloud.com/groups/what-ifblog-submissions.

\section{M}

Macahis, Feliz Anne Reyes. 2016. \#GYHAFY Tala. Editor: Barbara Lüneburg. Feb. 27. Accessed on Sept. 7, 2017.

https://what-ifblog.net/2016/02/27/gyhafy-tala/.

Marshall, Judi, and Geoff Mead. 2005. "Editorial: Self-reflective practice and first-person action research.” (Sage Publications) 3 (3): 235-244.

http://journals.sagepub.com/doi/10.1177/1476750305055999

McCracken, Grant. 2013. "Spreadable Media." In “Consumers” or "Multipliers"?

Henry Jenkins, Sam Ford and Joshua Green. Accessed on Sept. 11, 2017. http://spreadablemedia.org/essays/mccracken/\#.VssqGilCyGh.

Mersch, Dieter. 2015. Epistemologien des Ästhetischen. Denkt Kunst. Zürich: diaphanes.

Miebach, Bernhard. 1991. Soziologische Handlungstheorie. Eine Einführung.

Wiesbaden: VS Verlag für Sozialwissenschaften.

https://doi.org/10.1007/978-3-322-95676-7

Montrey, Clio. 2014. Call for Entries | Catrinas. Editor: Barbara Lüneburg. Oct.

27. Accessed on Sept. 9, 2017.

https://what-ifblog.net/2014/10/27/call-for-entries-catrinas/.

-. 2014. Call for Entries / Contemporary Fairy. Editor: Barbara Lüneburg. Aug.

14. Accessed on Oct. 1 2017. https://what-ifblog.net/2014/08/07/call-

for-entries-fairy-of-the-four-temperaments.

-. 2015. Call for Entries / What Gets Your Creativity Going? Editor: Barbara

Lüneburg. March 14. Accessed on Oct. 24, 2017.

https://what-ifblog.net/2015/03/14/call_for_entries_creativity/.

-. 2015. Call for Entries: Holiday Gifts / \#WhatIfSoundSamples. Editor:

Barbara Lüneburg. Dec. 18. Accessed on Oct. 27, 2017. 
https://what-ifblog.net/2015/12/18/call-for-entries-holiday-gifts/.

-. 2014. Catrinas / Submissions and Thoughts. Editor: Barbara Lüneburg. Nov. 7. Accessed on May 23, 2017.

https://what-ifblog.net/2014/11/07/catrinas-submissions-andthoughts/.

-. 2014. Creativity is Contagious, Pass it On. Editor: Barbara Lüneburg. Oct. 17. Accessed on Oct. 21, 2017.

https://what-ifblog.net/2014/10/17/creativity-is-contagious-pass-it-on/. —. 2016. \#WhatIfSoundSamples / Denim Szram. Editor: Barbara Lüneburg.

March 2. Accessed on Sept. 9, 2017.

https://what-ifblog.net/2016/03/02/whatifsoundsamples-denimszram/.

Münker, Stefan. 2009. Emergenz digitaler Öffentlichkeiten - Die Sozialen Medien im Web 2.o. edition unfeld. Suhrkamp.

Mughnetsyan, Anahit. 2014. "Armenia-Homeland" by Anahit Mughnetsyan.

Editor: Barbara Lüneburg. April 27. Accessed on Oct. 1, 2017.

https://what-ifblog.net/2014/04/27/armenia-homeland/.

Murchison, Julian M., and Curtis D. Coats. 2015. "Ethnography of Religious

Instants: Multi-Sited Ethnography and the Idea of "Third Spaces"."

Religions (Directory of Open Access Journals) 6 (3): 988-1005 (18).

$\mathbf{0}$

O`Reilly, Karen. 2009. Key Concepts in Ethnography. SAGE Publications.

https://doi.org/10.4135/9781446268308

ORF. 2013-2015. Personal Soundscapes. ORF musikprotokoll im steirischen

herbst. Accessed on Oct. 12, 2017.

http://personal-soundscapes.mur.at/en.

$\mathbf{P}$

Pink, Sarah. 2009/2015. Doing Sensory Ethnography. 2nd. London: SAGE

Publications. https://doi.org/10.4135/9781446249383

$\mathbf{R}$

Reckwitz, Andreas. 2002. "Toward a Theory of Social Practices. A Development in Culturalist Theorizing." European Journal of Social Theory (SAGE journals) 5 (2): 243-263. https://doi.org/10.1177/13684310222225432

$\mathbf{S}$

Sandiford, P. J. 2015. "Participant Observation as Ethnography or Ethnography as Participant Observation in Organizational Research.” In The Palgrave Handbook of Research Design in Business and Management, Herausgeber: K.D. Strand, 411-446. New York: Palgrave Macmillan. 
https://doi.org/10.1057/9781137484956_22

Sani, Margherita, Bernadette Lynch, Jasper Visser, and Alessandra Gariboldi. 2015. Mapping of practices in the EU Member States on Participatory governance of cultural heritage to support the OMC working group under the same name (Work Plan for Culture 2015-2018). EENC Short Analytical Report, European Expert Network on Culture (EENC), EENC.

Savin-Baden, Maggi, and Claire Howell Major. 2013. Qualitative research: the essential guide to theory and practice. Oxon: Routledge.

Schön, Donald A. 1983. The reflective practitioner: how professionals think in action. 1983: Basic Books.

Schatzki, Theodore R. 2002. The Site of the Social: A Philosophical Account of the Constitution of Social Life and Change. University Park: The Pennsylvania State University Press.

Shapiro, Dan. 1994. "The limits of ethnography: combining social sciences for CSCW." Editor: J.B. Smith, F.D. Smith and T.B. Malone. Proceedings CSCW' '94. Proceedings of the 1994 ACM Conference on Computer Supported Cooperative Work. New York: ACM. 417-428. https://doi. org/10.1145/192844.193064

Sherlock, Sarah. 2015. I won a contest for Sound-Design Composition. Sept. 28. Accessed on Feb. 25, 2016.

http://www.sarahsherlockmusic.com/\#!I-won-a-contest-for-SoundDesign-Composition/tkry5/56ogaoc4ocf25fa7fe1b92fc.

Simon, Nina. 2010. The Participatory Museum. Santa Cruz: Museum 2.o. Sweeney, Jason. 2012. Stereopublic. creative producer: Martin Potter. Accessed on March 17, 2016. http://www.stereopublic.net/\#what-is-it.

\section{$\mathbf{T}$}

Tacchi, Jo, Joann Fildes, Kirsty Martin, Kiran Mulenahalli, Emma Baulch, and Andrew Skuse. 2007. EAR / Ethnographic Action Research Training Handbook.

Queensland University of Technology. Accessed on June 26, 2017.

http://ear.findingavoice.org/index.html.

Tchiba, Martin. 2017. "Tchiba in Facebook-chat with the author." Facebook. July, 22.

Tinapp, Sybilla. 2005. "Visuelle Soziologie - Eine fotografische Ethnografie zu Veränderungen im kubanischen Alltagsleben." University of Konstanz. August. Accessed on Nov. 12, 2017.

http://nbn-resolving.de/urn:nbn:de:bsz:352-opus-19477.

Travers, Max. 2001. Qualitative Research through Case Studies. London: SAGE Publications. https://doi.org/10.4135/9781849209724

Troxler, Peter. 2011. Open Design Now - a collaborative effort of Creative Commons Netherlands, Premsela, the Netherlands Institute for Design and Fashion and Waag Society. June 2. Accessed on May 30, 2014. 
http://opendesignnow.org/index.php/article/libraries-of-the-peerproduction-era-peter-troxler/.

Turner, Stephen P. 2014. Understanding the Tacit. New York: Routledge.

\section{U}

University of Glasgow. 2017. University of Glasgow - College of Arts - Research

Ethics Policy. Accessed on July 6, 2017.

http://www.gla.ac.uk/colleges/arts/research/ethics/ethicspolicy/.

\section{V}

Van Maanen, John. 1988/2011. Tales of the Field. On Writing Ethnography. 2nd. Chicago: The University of Chicago Press.

\section{W}

Wacquant, Loïc. 2005. "Carnal Connections: On Embodiment, Apprentice ship, and Membership." Qualitative Sociology 28 (4): 445-474. https://doi.org/10.1007/s11133-005-8367-0

Weber, Eckhard. 2016. Ultraschall Berlin - Festival für neue Musik. Rundfunk Berlin Brandenburg. January. Accessed on June 15, 2017. https://ultraschallberlin.de/aufsatz/louis-aguirre/.

Weidenbaum, Marc. 1992-2017. Disquiet ambient electronica. Accessed on Oct. 10, 2017. http://disquiet.com/2013/04/25/disquiet-junto-faq/.

Wosnitzka, Susanne. 2017. Comment on Facebook.

http://tinyurl.com/y7hcmelz, Aug. I9. Accessed on Feb I9, 2018.

Wosnitzka, Susanne, Anahit Mughnetsyan, and Gloria Guns. 2017. Comment on Facebook. April 26. 



\section{AUTHORS}

Main Author:

Barbara Lüneburg, Dr. phil, is an internationally acknowledged performing artist and artistic researcher working in contemporary classical art music and multimedia art. From 2014 to 2018 she was director and lead artist of TransCoding. Her previous arts-based research deals with collaborations between performers, composers and music promoters, charisma, performer-audience relationships and game-based audiovisual art. Lüneburg holds a professorship for Ensemble and Digital Performance at the Trossingen University of Music, Germany, and works as researcher at the University of Music and Performing Arts Graz, Austria.

www.barbara-lueneburg.com

Contributing Authors:

Torsten Flüh, Dr. phil, interviewer, is a researcher on literature, media, social media, internet and journalism. He investigates language, knowledge and science as they operate on the internet, while actively practising his research on his own blog NIGHT OUT @ BERLIN. Flüh has taught literature, blogging and journalism at the Humboldt-University of Berlin and the Philipps-University in Marburg. Before 2001, he worked as a lecturer at the University of Hamburg, the Fudan University, Shanghai, Beijing Foreign Studies University and University College Dublin, National University of Ireland.

Kai Ginkel, Dr. phil, co-author of part III, was junior researcher on the project TransCoding within the Institute of Music Aesthetics at the University of Music and Performing Arts Graz, Austria. He studied sociology, psychology and political science at the Technical University of Darmstadt. From 2012 until 2015, he was a Doctoral candidate in the 'Sociology of Social Practices' programme at the Institute for Advanced Studies, Vienna. In 2016, Ginkel earned his PhD on the topic Noise: A Praxeological Approach to Sound at the Catholic University of Eichstätt-Ingolstadt. His thesis was published by Transcript in 2017 . 
Interviewees:

Kai Ginkel (biography above)

Barbara Lüneburg (biography above)

Clio Montrey, BMus MA, is a creative director, musician and writer, and was the social media manager for TransCoding. The recipient of several musical awards and distinctions, Montrey graduated from both composition and performance programs from McGill University, the University of Music and Performing Arts Vienna, and the Conservatory of Vienna. She specialises in multimedia and has participated as an artist in numerous interdisciplinary projects. Her personal artistic practice enriches her understanding of social media in the context of the arts.

\section{WEBSITES}

Project website:

http://transcoding.info

Complementary website with annotated audio and video excerpts:

http://transcoding.info/english/book.html 


\section{INDEX}

A

academisation of art, 164

access

equal, 43

open, $43,53-54,56-57,60,104$

restricted, 55

to (research) knowledge, 132-133

to (uncensored) Internet, 42-43, 54

to a field, 135

to decision-making, 43

to information, 16o, 162

to knowledge, 51, 56, 140, 162, 168

to meaning, 135,166

to the project, $18,57,104$

Adler and Adler, 136

aesthetic

affective, 165

approach, 78,120

choice of, 33

crossover, 16, 27

decision making, 124

defying aesthetic class thinking, 32

experience, 131

frictions, 104

inspired by community, 69

knowledge, 130

of social media, 32

rules, 144

spontaneous, 31

standards of beauty, 141

territory, 68

affect,

construction of, 144

agency

active, 119, 124

degree of, 107

given, 107 of our community, 100

sense of, 159

to follow, 56

Aguirre, Louis, 139-152, 156-157

algorithms

influence of, 46

used by social media for distribution, 43, 46-47

Alvesson, Mats, 135, 158, 160

Anderson, Nels, 134

anthropology, 133

visual, 166

Armenia, 83-85, 154

artefacts

artistic, 132

artworks, 135

culture expressed through, 166

discursive, $15^{8}$

non-human, 135

artist-audience relationship, 116, 120,124

artistic research, 7, 9, 17-18, 23, 50,

$127-133,135-137,140-142,145$

$149-151,153-157,159-168,172-$

173

arts-based inquiry, 130, 141

arts-informed inquiry, 130

arts-informing inquiry, 130

practice-based, 129

practice-led, 129

artworks by the community

Feathers, Laura

Soundscape 1, 93

Guns, Gloria

Fan Death, 75, 106, 119, 175

Michelle, Daniela

Catrina, 99, 156 
Szram, Denim

What iffree soundsample, 94

Ulubeanu, Sabina

Man in Abandoned House, 31

Old Woman Behind Window, 96

Vales, Maria Baez

Shelfie, $6_{4}$

artworks of TransCoding

Mad Maddy, 70

Soundwaves, 70

Souvenir de Budapest, 70

The Gods Envy Us, 70, 99-100.,

153,156

They Speak in layered..., 70

What if we had wings?, 23-24, 56 ,

62, 67-68, 107-109, 110, 113

artworks, various

Blind Tapes, 25

Disquiet Junto, 25-26, 35

Personal Soundscape Project, 24

Stereopublic: crowdsourcing the

quiet, 24

Toque a Eshu y Ochosi, 139 - 145,

147-148, 159

Walk that sound, 25

WIReless - Ein Social-Media-

Klavier-Recital mit Martin

Tchiba, 26

audience participation, 15

audience response, 133

audio art, 24

audio entries, 92, 95

audiovisual installation, 14, 17, 21,

$42-43,57,76,101,104$

Austin and Forinash, 130

Austrian Science Fund, 7, 15, 170-

171,175

authentic

use of community material, 21, 23,

$37,59,69,110$

authenticity, 19, 21, 48, 111

authority

aesthetic, 58

afforded, 55, 101-104

area of conflict, 108-109, 114

challenged, 31

creative, 43

curatorial, 26 degree of, 104-105

distribution of, 52

exclusive, 173

exercised, 116

in decision-making, 57-58, 104-105,

120,124

in participatory art, 7, 100

in the creative process, 16

minimum given, 107

permeability of, 27

position of, 133

preconceived, 21

shared, 43, 57, 101, 163

sign of, 50

unlimited, 114

autonomy, 70

sign of, 55

avant-garde pop music, 12

\section{B}

being native, 136, 147, 159, 162

belonging, $12,75,76$

belongingness, 74, 78

Bishop, Claire, 24, 51

blog categories

Art we love, 10, 6o-61

Identity, 10

Making of, 10, 21, 59, 113

You, Us and the Project, 10, 14, 60 blog posts

Athena - Why I Like the Ancient Goddess, 153

Catrinas / Submissions and Thoughts, 99, 153

Lines of Connection and Identity, 153,155

Making of: Vienna Weekend Thoughts, 77

Masks for a New Identity, 153

Rose Petals, Queer Trauma, and Rituals - AA Bronson's Sacre du Printemps, 153

Te Reinga...the Leaping Place of Spirits, 153, 154

The Orichas of Santería, 153

Boddy, Jennifer, 160-161

bodily and discursive activities, 143

Borgdorff, Henk, 132-133, 160,

165-166 
bots, $42,45,46$

brand/branding, 41, 68

brand image, 114

self-branding, 162

brought self, 20-21

Bueger, Christian, 135

Butler, Judith, 9, 13, 71, 81

C

Call for entries

\#GYHAFY - Give yourself a holiday away from yourself, 70 , 79, 85-92, 100, 124

\#WhatIfDrone-Remix Contest, 75 , 78-79, 94

Catrinas, 95, 98-99, 122, 153, 156

City Hopping for a New Identity the Sky is No Limit, 62

Contemporary Fairy, 100, 108-109

Creativity is Contagious, 63, 97, 156

Frames of Life, 38

Holiday Gifts 1

\#WhatIfSoundSamples, 92-93

Powerful Woman, 39-40, 45, 73

What Gets Your Creativity Going?,

$69,73,78$

Who is Damian, Lia and

Barbara...?, 62

Window shopping for a new

identity. Vienna. Spring 2014,

61

Calls for entries

competition, 79

intensities of engagement, 72

photos, sounds, stories, 37

responses to, 57,80

Canadian Charter of Rights and

Freedoms, 71, 73, 95

capability development, 119, 122,

125

Caroll, Lewis, 97

Catholicism, 146, 149, 152-153

Catholic All Souls' Day, 98

Catholic Church, 146-147, 152

Catholic priests, 147

Catholic religion, 146

Catholic saints, 147, 156
Catholic upbringing, 147-148

practising Catholic, 139

Roman Catholic belief system, 146

Catrinas, 95, 98-99, 156

Chicago School of sociology, 46

Chicago School of symbolic

interactionism, 134

claim to knowledge, 139, 163

classical music, 9, 12-13, 24, 32, 76,

$149,157,171-172$

classical violin, 32

classical Western European music, 34

closeness to the researched subject, 136

closure of the field, $16_{3}$

Coffey, Amanda, 136

cognition, 130-131

Cohn, Carol, 134

commenting, 43, 55, 57, 72, 119,

125

communication, 9, 12

authenticity of, 111

barriers of, 58

platforms, 18

direct, 36, 121

establishing of, 120

means of, 36

network-based communication, 76

online-communication, 78

platforms, 43

strategies, 23

structures, $81-82$

through the arts, 172

social media as tool of, 52

unrestricted, 56

via virtual channels, 49

worldwide communication, 35

community

activity of, 120

agency of, 82, 100

approval of, 125

authority of, 12, 100-101, 106-107,

114

building of, 16, 27, 67, 113

close-knit, $5^{8}$

community creation, 51 
community feeling, 75

community gallery, $42,45,53,63$,

77,80

community spirit, 93

creative voice of, 172

decision-making power, 104

dialogue with, 56, 120

discourse with, 24

frictions with, 104

heterogeneous, 66, 74

influence of, 62, 64, 78, 117

interaction with, 9, 16-17, 42-43,

$$
56,65,100,111,122,169
$$

online, $49,51,52,72,78,108$, 111-

$112,169,173$

participation of, $7,10,66,110$

participatory community, 100

portrayed community members, 105

praise from, 110

problems working with, 111, 113

production of artwork with relation

to, 121

self-determination of, 122

social media community, 21, 122

tight-knit, 35

competence, 74-75, 152

connectedness, 74, 78

construction of 'realities', 168

constructivism, 133

contemporary art, 9, 16, 33, 50, 53

classical contemporary multimedia

performances, 54

classical contemporary music, 18 ,

$$
\text { 6o, } 150,152
$$

contemporary art music, 9-10, 12,

$58,65-66,68,83,122,150,169$,

170

multimedia art, 7, 9-10, 24, 58, 60,

$$
66,81,120,169
$$

multimedia environment, 36

multimedia performance, 21,28

contributions, remix, 60, 62, 69, 72, 74-76, 78-79, 82,93

text, 11, 93, 95, 97-98, 101-102, 104-105, 108, 110

self-expression via creative contributions, 87, 99, 120

contributors

Allain, Sandra, 109

Beat S, 94

Bonshek, Corrina, 94

Bushnaq, Suad, 40

Feathers, Laura, 93, 121

Green, Anthony, 73, 87, 90-92, 124

Guns, Gloria, 47, 64, 71, 75-76, 78, 81-82, 95, 101, 105-106, 119, 123, 171, 175

Macahis, Feliz Anne Reyes, 87-90

Magic Sun, 93-94

Mateus, Ricardo Tovar, 104, 106 121

Michelitsch, Katarina, 30

Michelle, Daniela, 99, 156

Mughnetsyan, Anahit, 62, 81, 83$85,121-122,122,154,158,171$, 175

Murzakhanova, Alina, 97, 102-103, 105, 121

Ohanyan, Heghine, 83, 171

Rey, Hali, 97

Spitaler, Ernst, 68

Sqalli, Malika, 147, 149, 168

Szram, Denim, 93-94

Taft, Sharlea, 63

Ulubeanu, Sabina, 30-31, 95-96,

121

Vales, Maria Baez, 64-65

Wosnitzka, Susanne, 79-81, 92

Zyla, Monika, 71, 73

Cook, Nicholas, 150-151

Cox, Arnie, 142

Creative Commons

the idea of, 46 
Creative Commons agreement, 37

Creative Commons licence/license,

34, 53, 90, 93, 117

Creative Commons network, 45

creativity

act of creation, 79, 83

democratised, 34

furthering of, 36

mass creativity, 77

potential for, 12

stimulating of, 36,170

taking away from, 49

triggering of, $69,74,120$

unlocking of, $17,37,51$

via social media, 36

crossover (art), 16-17, 58, 83, 116,

173

crossover culture, 171

crowdsourcing, 24, 55

cultural resources and reference

points, 139, 145

cultural science, 9

cultural studies, 46

D

data analysis, 133

data collection, 127-129, 133, 137,

$140-143,159-160,166$

data generation, 133

Deci and Ryan, 74, 78

decision-making in art, 26

deep membership, 136

Deleuze, Gilles, 165

democratic affordances, 53, 57, 66

Derrida, Jacques, 165

Día de los muertos, 98, 156

Dietrich, Alex, 39

Diggle, Keith, 54

digital audio software, 34

digital audio workstation, 55

digital natives, 11, 54

discontinuities, 139, 159, 165, 169

discourse 127, 148, 159-168 influence on, 57

interdisciplinary, 53

on identity, 60

production of, 129

shared, 9, 122

verbal, 130

diversity, 32, 60, 73, 157

doing ethnography, 134

doing with things, 135

\section{E}

ego gratification, 79, 81

embodiment, $140-142,159$

emotional response, 74,81

empowerment, 170

digital, 42

female, 40

of people in their creativity, 43

personal, 12, 81, 173

through social media, 42

value of, 107

equality, 43, 53, 56, 59, 82, 90, 95, 99, II6, I35

ethical guidelines/considerations, 50, $139,159-164$

ethnographic sociology, 17, 23, 50, $127-129,133-138,164$

ethnography, 15, 159-164, 166, 168

at-home ethnography, 135

insider ethnographies, 162

Marxist, 133

sensory, 133, 152-153, 160-161

165-167

Weberian, 133

writing process, 134

European avant-garde music, 121

European Researchers' Night, 22, 109-110

evaluation, 55, 57, 66, 119, 127, 133 , 138,168

exclusion, 129,163

experience

aesthetic/through art, 131-132, 142,

$144,150,165,173$

multisensoriality of, 137

(multi)sensory, 132, 152

religious, 141, 143, 147-149, 152-153,

154,157 
ritual, 143-144, 146-147, 149

shamanistic, 147-148, 152

spiritual, 152-153, 156-158

extended (instrumental) techniques, $116,121,150,171$

$\mathbf{F}$

Facebook, 21, 26, 29, 32, 37-38, 41-43, $47-48,52-53,57,59,74-76,78-79$,

$81,92,95,100,121-124,173$

Facebook event, 48

February Album Writing Month

(FAWM), 21-22, 35, 118

field notes, 5०, 159

fieldwork, 18, 127, 132, 133-134, 140, 159, 166

Flüh, Torsten, 27ff., 188

folksonomy, 55

Foucauldian, 158

Foucauldian discourse analysis, 17, 127, 139

Foucault, Michel, 127, 129, 139, 148, 163, 165,168

free sound sample, 53, 93-94

\section{G}

Gangnam Style, 12, 32

Gauntlett, David, 51, 87

Geertz, Clifford, 107, 134

gender, 9, 13-14, 29, 70, 85-87, 90

(undoing) gender, 6o, 65, 70, 89,

92, 110

domain of agency and freedom,

13,85

perform the gender script, 13

performativity, 13

performing/performative gender,

13,85

self-chosen, 85

genius,

composer, 34

creative, $58-59,120,121$

isolated, 35

lonely, 34, 48, 51

Ginkel, Kai, 9, 15-16, 23, 28ff., 127 ff. 187

Gobo, Giampietro, 134-135, 137

Gods of Santería

Babalú Ayé, 145
Echu, 140, 145

Juan de la Juan tu, 145

Ochosi, 139-140, 143, 145

Olofi, 141

Goffman, Erving, 137, 151

going native, 135, 147, 159, 162

guest blogs

Armenia-Homeland, 62, 83-84, 154

Johnny, 70, 80, 92

piargno, 87, 90-92

Tala, $87-90$

H

Harvard University, 137, 166

hedonic rewards, 79, 81

Hegner, Victoria, 135

Heidegger, Martin, 166

Hesse, Hermann, 115

highbrow art, 59

Hughes, Randall and Shapiro, 133

human rights, 30, 47, 60, 73, 87, 95,

134, 175

identity, 10, 13-14

and social media, 46-47

and spirituality, 153

art identity, 81

artwork on, 77

boundaries of, 174

concept of, 10, 14

constructed, 160

corporal identity, 144

creative, 6o, 63, 93, 97, 100

established in interactions, 46

establishing (of) identity, 149, 151

expressing/expression of, $46,80-83$,

151

facets of, 70

folk identity, 98

homeland, 83

imagined, 86

in search of, 28

meaning of, 84

mortality, 99

musical, 94, 150

musically established, 139

notions of, 157 
of a people, 154

personal, 18, 46, 95, 155

related to community members, 121

relevance of identity to community,

29

representation of, 83,149

roots of, 83,154

self-chosen, 85

shared, 78

spiritual, 157

topic of, 44,56

visions of, 70

Imaginarium, 36

incentives

denial of, 100

to compose, 26

to create, $37,58,82,172$

to express oneself, 53

to participate, $16,43,58-59$

inclusive, 58

indexicality, 145

influence

from new music, 32

mutual, 9, 107, 120, 170

of researchers on researched field, 128

of social media on the aesthetic of the artworks, 29-30

of the community on the evolving artwork, 9, 11-12, 18-19, 49, 51, 53, 56-57, 66-71, 82-83, 95-116, 117, 120-121

Ingold, Tim, 136-137, 147

Instagram, 29, 53, 59, 80, 95

interactionism

symbolic interactionism, 134

interactionist, 134

interactionist research, 135

interdisciplinary approach, 133, 167

\section{J}

Jank, Sabine, 53-54, 56, 113

Japanese Bon Festival, 98

Jenkins, Henry, 51-53, 117

K

Kaplan and Haenlein, 29, 42, 82

knowledge, 52, 135 aesthetic/through art, 130-132, 139,

$142,161,165,172-173$

claim to, 127-129, 159, 163

construction of, 7, 127, 159

embodied, 143-144, 165

gain of, 125, 130-132. 159, 165, 169

production of, 54, 133, 159, 162 -

166

spiritual, 157

Korean Chuseok, 98

Korean lullaby, 76

K-Pop, 9, 12

Kruse-Weber, Silke, 131

Leadbeater, Charles, 77-78

Lenette, Caroline, 159

Levinas, Emmanuel, 165

liking, 43, 57, 72, 119

Luka Ivanovic, 25

Lukatoyboy, 25

\section{M}

making/rendering the familiar strange, 134,159

Māori, 153-155

Marshall, Judi, 167

materiality, 129, 135, 140

Mead, Geoff, 167

meaning

construction of, 150, 159

evaluation of, 168

meaning making, 128, 137, 147, 159, 165

media competence, 43

Mersch, Dieter, 130

method of compassion, 135

methodologies, 127-129, 136,

collaborative, 161

development of, 167

visual, 160-161, 166

Mexican Day of the Dead, 98

micro-blog, 29

micro-blogging, 42, 52

mimetic

mimetic engagement, 142

mimetic invitation, 142

mimetic motor action, 142 
Mimetic Motor Imagery (MMI), 142

mimetic participation, 142

mirror neurons, 141-142

Molle, Andrea, 134

Montrey, Clio, 15, 19, 20-22, 28-50, 52, $63,69,75-76,93,98-99,104,-105$, $108,125,175,188$

motivation

(cultural-)political, 72-73

extrinsic, 73-75, 78, 81

for participation, $17,58,68,72-82$, 102,120

hedonic rewards, 75

intrinsic, 73-75, 78, 81

to create, 122

multipliers, 119

Münker, Stefan, 53-54

Murchison and Coats, 157

music business, 41

musical multimedia, 150

\section{N}

narrative, $33,57,66-67,70,80,83,139$, $145,147-148,152,160,166,171$

National Union of Women's Suffrage

Societies, 104

node within a network, 139, 148, 157

Nono, Luigi, 12

\section{$\mathbf{0}$}

O'Reilly, Karen, 162

Obbá, 141, 144

objectivity, 132, 162

observation, 134-135, 137, 142

open source, 34, 37, 73, 82

opportunistic complete membership,

136

ORF, 24-25

organisational change, 119, 121

\section{P}

participant involvement, 161

participant observation, 50, 72, 134, 140, 147,159

participation, 9-10, 15, 66, 72, 81

active involvment, 72

appointment-based, 117

autonomous creative engagement, casual engagement, 72

co-creative, 56, 104

collaborative, 56

constraints to, 55

contributory, 26, 56, 104

democratic, 26

engagement-based, 117

forms of, 59

growing, 110

in art, 12

in the field, $135-136$

limitations of, 54

mode of, 19, 59, 101

model of, 18, 56, 72

of fieldworker in the studied field,

133

of the researched field, 168

online, 17

open to everybody, 18

passive following, 72

potential for change, 101-125

potentiality of, 56

repeated, 72

strategies of, 54

sustained engagement, 72

threshold to, 52

user participation, 55

via social media, 43

via web 2.0, 51, 56

participative/participatory art(work), 7 , $13-14,16,18,24,42-43,48-51,81$,

100, 107, 110, 169-170, 173

participatory culture, $7,9,13,15-16,26$,

$34,51,52-53,58,77-78,113,120$

participatory process, 111

participatory public, 53

participatory set-up, 110, 114

peer group, 12, 23, 33, 50, 66, 68, 74-75,

$81,113-114,122,164$

peer recognition, 79-80, 107

performative, 13, 18, 144, 147

performer, 15, 21, 23-24, 49, 68, 79, 90,

$139-145,139,169,172,176$

permeability, 9, 27, 43, 57, 107, 170

phenomenology, 133

philosophy of conflict, 56

Pink, Sarah, 137, 152-153, 160-161, 167

Pinterest, 53, 69, 95

political science, 133, 187 
popular culture, 9, 11-13, 32-33, 54, 106, 116,150

Posada, Jose Guadalupe, 98

post-modernism, 133

post-structuralism, 133

potential

epistemic, 147

for change, 101-117, 119-120

power

administrative power, 44

altering traditional power

relationship, 121

decision-making power, 104

dominant power relations, 164

exploratory power, 128,133

female power, 40

power change, 120, 124

power constellations, 56, 113

power/procedures of exclusion, 163

power of rites, 147

power of transformation, 153

power relations/relationship, 12, 68 ,

$127,159,163-164$

power relations artist-audience, 116 ,

$119,121,124$

power strategies, 17,128

power structure, 107

question of legitimacy, distribution

of money and reputation, 164

sharing of, 113

practices

artistic practice, $7,9,12-13,18,131$ -

$132,144,147,164,170$

arts practice, 17-18, 125, 127, 129,

$132,142,147,160,162,164,169$

bodily practice, 144

common creative practices, 73

creative practice, 100

discursive practices, 128

dissolution of traditional practices, 56

ethnographic media practices, 137

everday practices, 46, 134

explorative practices, 168

methodological practices, 128

network of practices, 149, 161

of observation, 168

of Santería, 141-142, 150 online practices, 32

participatory practice, 119

performance practice, 35, 140, 143 ,

145

political practices, 42

arts practice, $125,127,129,132,142$,

$147,160,162,164,169$

practices of engagement, 72

practices of social media users, 67

religious practice, 140, 144, 149, 157

social practice, 135, 140, 151

spiritual, 148

traditional practices, 56, 113

transcoding of cultural practices, 34

pragmatism, 133

praxeological approach, 140

praxeology, 127, 135, 157

preparation, incubation, insight and

evaluation, 59, 66

pressure

financial, artistic and aesthetic, 164 process

artistic research process, 141

communication process, 18, 112

creative process, $9-10,18,27,31$,

$34,37-40,43,48-49,58-60,66$,

72, 93, 107, 132-133, 147, 152,

160,170

decision-making process, 100, 120

ethnographic process, 137

participative process, 140

truth-seeking process, 139

PSY, 9, 12, 32

psychology/psychological 17, 51, 74,

133184

Q

quality standards, 68, 167

\section{$\mathbf{R}$}

Ramachandran, Viliayanur, 141

Read me, 17, 19, 42-43, 47, 51, 53, 57, 70, 72, 76, 81, 95, 97-98, 101-107, 114$116,119,123,169,171,173,175$

Reckwitz, Andreas, 135, 144

recognition, 78-81, 107, 121

reference points

cultural reference points, 145,148 
personal reference points, 149

Reinharz, Shula, 19

relatedness, 74, 76, 78

relevance, 39

remix, 12, 32, 60, 62-63, 69, 72, 74-76, 78-79, 82, 93-94, 105-106, 117, 119, 171,175

representation

artistic expression of findings, 130 , 161

crisis of, 137

ethnographic writing styles, 137

exploratory power, 128

informative value, 128

informative value of an artwork, 133

of research findings, 159, 167

of research results, 130, 132

textual interpretations of findings, 160

verbal description, 141

verbal report, 161,165

visual perception, 141

written report, 166-167

reputation, $79,113,121,164,170$

research perspective

active involvement, 159, 161

immediate involvement, 130, 141

inner perspective, 162,164

inside perspective, 130, 141, 162, 172

inside position, 132

participants' perspectives, 161

praxeological perspective, 142-143,

149

sociological perspective, 148

respect(ful), 21, 23, 37, 42

return, 42

rites

Christian, 146, 149

explored on the blog what-ifblog.net,

$153-54$

multi-sitedness of, 158

of Santería, 146, 153

of the Catholic Church, 147

shamanistic, 147, 148

ritual(s), 28, 143, 153

access to, 135

bodily enacted, 144, 145-146, 148

of the concert, 144 power of, 146-147

production of, 148

religious, 144,148

Santería, 141, 146

shamanistic, 148

shared between Santería and

Catholicism, 147

Robert H. McKim, 36

rules, 44-45, 127

aesthetic, 144

privacy, 113

religious, 153

RUUKKU, 8, 100, 176

\section{S}

Salomonsen, Jone, 135

Sandiford, P.J., 19

Sani, Margherita, 119-120, 125

Santería, 128, 139-142, 144-153, 157

Savin-Baden and Howell Major, 129-130, 133

Schatzki, Theodore R., 135

Schmiede Hallein, 115

Schön, Donald A., 162

self-censorship, 162

self-concept of the artist, 17, 68, 101, 170

self-determination, 119, 122, 125

Self-Determination Theory (SDT), 51, 74

self-expression, 52, 87, 99

self-promotion, 41, 72

self-reflective/self-reflectivity, 132, 160163,166

Shaman ceremonies, 139, 145-146

Shapiro, Dan, 133

sharing, 12, 21, 29, 41, 43, 48, 50, 52-55,

$82,117,119,122,125$

shelfie(s), 63-64, 77, 95, 97-99

Simon, Nina, 26, 51, 56, 104

situational self, 19, 21

Slices of Life, 7-8, 13, 17, 19, 21, 28-30, $32-34,37,40-42,47,53,56-57,66$, 70, 72-73, 76-79, 80-83, 92, 95, 98 , 100, 120, 128, 142, 146, 149, 169, $171,173,175,166,168$

- Charter of Rights and Freedom, 73, 95, 175

- I Am a Priest, 17, 83, 127-128, 137 , 139-159 
- Johnny, 70, 80, 92

- Korean Lullaby, 76

- My Chosen Name is, 70, 92

- The Shirt, 28-30

- Who Am I?, 98

thematic development of, 66 social anthropologist, 136

social constructionism, 133

social dimension, 58,61

social interaction, 58,142

social media

and composing, 35

and participatory culture, 7

as communication tool, 15, 20, 43,

120

as political activity, 42-43

censorship, 43

content strategies, 16, 51, 58-65

Creative Commons, 44

creativity, 49

frameworks of, 46

hate speech, 44

personal interaction via social

media, 76,122

specifics of different types, 29

structure of, 113

templates of different platforms, 43

tool in the process of creation, 41

users (tech-savvy), 54, 63

viral, 12, 33

social media manager, 19, 41, 47

social sciences, 51, 136, 153, 160

sociology,

folk sociology, 136, 147

sound design, 121, 150, 152, 171

SoundCloud, 21, 26, 34-35, 37, 42, 53,

6o, 69-70, 72-75, 92-94, 98, 105,

$117-119,125$

SoundCloud group, $35,44,57,75,78$,

80,93

soundscape, 24-25, 93-94, 151

spirituality, 62, 83, 98, 139, 149-150,

$152-153,156-158$

Standford University, 129

Steirischer Herbst

musikprotokoll, 25

story-telling, 32-33, 57, 70

subjectivism, 136, 147
Sweeney, Jason, 24

system of references, 139, 147

\section{$\mathbf{T}$}

taboo, 17, 129, 131, 135, 163

tacit knowledge, 143-144

Tchiba, Martin, 26

Thrasher, Frederic M., 134

Tinapp, Sibylla, 137

transparency, 42, 112, 137

Travers, Max, 134

truth, 17, 139, 159, 165

construction of, 127

search for, 131,165

Tumblr, 37, 42, 45, 53, 59-60, 63-64, 77,

$80,95,123$

Twitter, 21, 26, 29, 32, 38, 41-43, 46, 52-

$53,59,67,74,76,81,95,123$

\section{$\mathbf{U}$}

Ultraschall Festival, 28-29, 33, 122, 140, 142

University of Music and Performing

Arts, Graz, 15, 79, 92, 131, 187

\section{V}

value

added, 114, 121, 167

creating value, 117

for the artists, 114

independently generated, 119

learning value for participants, 107

of community submissions, 68

offering values, 61

social value, 107

values, 110

change of, 41

cultural, 51

of an academic field, 129

of artistic research and ethnographic sociology, 128

of the audience, 142

reflected in our project, 120

search for, 17

van Maanen, John, 134, 166

verifiability, 161

Virgin Mary, 146 
W

Wacquant, Loïc, 136, 147

web $2.0,7,9,16,18,51,55-56$

Weidenbaum, Marc, 25-26, 35

Wetherall, Margaret, 139

what-ifblog.net, 9-11, 14-15, 18, 28-29, 37, $42,45,54,56,58-62,70,72,74,78$ -

$80,93,109,122,153,170$

WordPress, 10, 13, 15, 21, 29, 43-44, 55,

59

writing groups, 49

www.transcoding.info, 17

Y

YouTube, 12-13, 60, 62-63, 67, 69-70, $77,80-81$ 


\section{Cultural Studies}

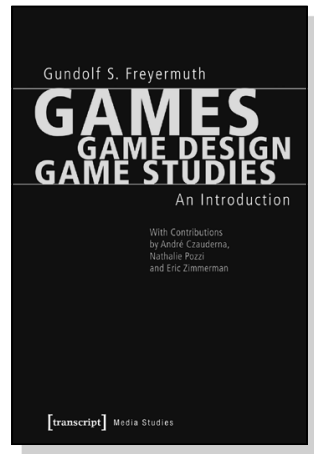

Gundolf S. Freyermuth

\section{Games | Game Design | Game Studies}

An Introduction

(With Contributions by André Czauderna, Nathalie Pozzi

and Eric Zimmerman)

2015, 296 p., pb.

$19,99 €(D E), 978-3-8376-2983-5$

E-Book: 17,99 € (DE), ISBN 978-3-8394-2983-9

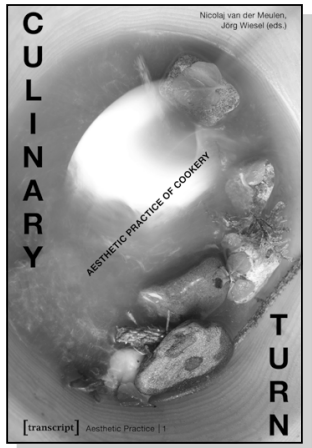

Nicolaj van der Meulen, Jörg Wiesel (eds.)

\section{Culinary Turn}

Aesthetic Practice of Cookery

(in collaboration with Anneli Käsmayr and

in editorial cooperation with Raphaela Reinmann)

May 2017,328 p., pb., col. ill.

29,99€ (DE), 978-3-8376-3031-2

E-Book: available as free open access publication

ISBN 978-3-8394-3031-6

\section{SUZI MIREANI}

TAREET MARILTS

INTERNATIONAL TERRORISM

MEETS GLOBAL GAPITALISM

IN THE MALL

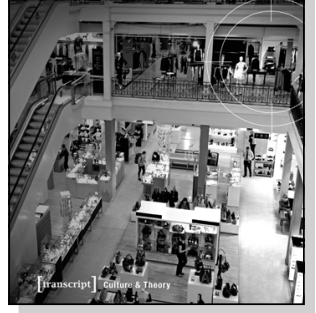

Suzi Mirgani

Target Markets -

International Terrorism Meets

Global Capitalism in the Mall

2016, 198 p., pb.

$29,99 €(D E), 978-3-8376-3352-8$

E-Book: available as free open access publication

ISBN 978-3-8394-3352-2 


\section{Cultural Studies}
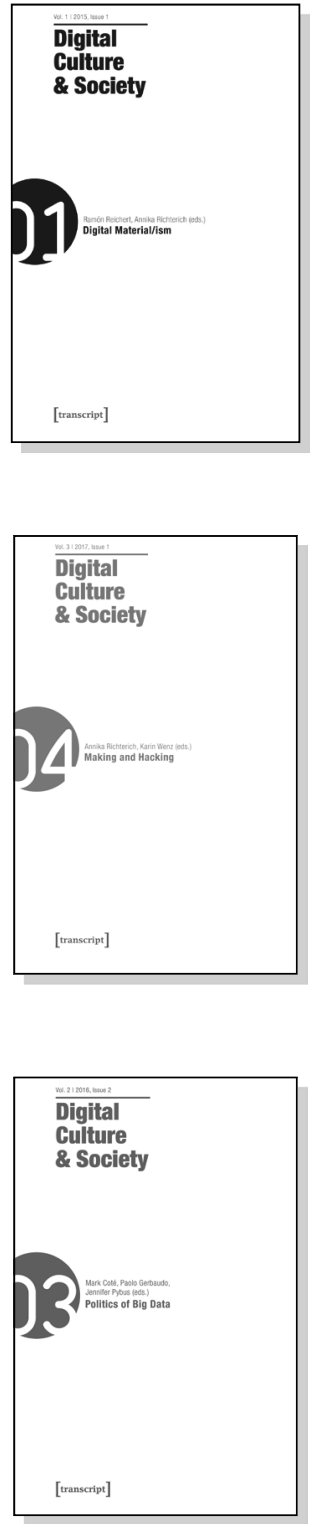

Ramón Reichert, Annika Richterich, Pablo Abend, Mathias Fuchs, Karin Wenz (eds.)

Digital Culture \& Society (DCS)

Vol. 1, Issue 1 - Digital Material/ism

2015, 242 p., pb.

$29,99 €(D E), 978-3-8376-3153-1$

E-Book: 29,99€ (DE), ISBN 978-3-8394-3153-5

Annika Richterich, Karin Wenz, Pablo Abend,

Mathias Fuchs, Ramón Reichert (eds.)

Digital Culture \& Society (DCS)

Vol. 3, Issue 1/2017 - Making and Hacking

June 2017, 198 p., pb.

$29,99 €(D E), 978-3-8376-3820-2$

E-Book: 29,99 € (DE), ISBN 978-3-8394-3820-6

Ramón Reichert, Annika Richterich, Pablo Abend, Mathias Fuchs, Karin Wenz (eds.)

Digital Culture \& Society (DCS)

Vol. 2, Issue 2/2016 - Politics of Big Data

2016, 154 p., pb.

$29,99 €(D E), 978-3-8376-3211-8$

E-Book: 29,99 € (DE), ISBN 978-3-8394-3211-2 